UnIVERSIDAD NACIONAL DE LA PLATA

FACULTAD DE HUMANIDADES Y CIENCIAS DE LA EdUCACIÓN

Secretaría de Posgrado

\title{
ACONDICIONAMIENTO FÍSICO \\ PRECOMPETITIVO PARA EL TENIS
}

Licenciado: Jefferson Cetina Pinilla

Tesis para optar por el grado de Magíster en Deporte

Director: Agustín Lescano, FaHCE-UNLP

La Plata, 15 de diciembre de 2014 
I. INTRODUCCIÓN. 5

$\begin{array}{ll}\text { 1.1. Tema. } & 7\end{array}$

$\begin{array}{ll}\text { 1.2. Problemática. } & 7\end{array}$

1.3. Objetivo general. 8

1.3.1. Objetivos específicos.

1.4. Metodología. 8

$\begin{array}{ll}\text { II. ESTADO DE LA CUESTIÓN. } & 10\end{array}$

$\begin{array}{ll}\text { 2.1. El calentamiento. } & 11\end{array}$

2.2. Estiramiento en el calentamiento. 12

$\begin{array}{ll}\text { 2.3. Acondicionamiento físico. } & 13\end{array}$

III. EL TENIS, SUS CARACTERÍSTICAS Y COMO SE HA TOMADO EL ACONDICIONAMIENTO FÍSICO Y EL CALENTAMIENTO EN ESTE $\begin{array}{ll}\text { DEPORTE. } & 17\end{array}$

3.1. La cancha de tenis y sus herramientas de juego. 17

3.1.1. La cancha de tenis y su red. 17

3.1.2. La raqueta. 18

3.1.3. La pelota de tenis. 19

3.2. El calentamiento y su tradición en el tenis. 20

3.2.1. Estiramiento en el calentamiento del tenis. 21

3.3. El acondicionamiento físico en el tenis. 22

3.4. Perfil fisiológico del tenista. 23

3.5. Movilidad en el tenis. 26

IV. ESTUDIO DE LOS MOMENTOS Y ACCIONES DEL TENISTA EN LA COMPETICIÓN. $\quad 28$

4.1. Desplazamientos del tenista en la competición. 28

4.2. Tipo de desplazamientos en el tenis. 32

4.2.1. Desplazamientos hacia adelante. 33 
4.2.2. Desplazamientos laterales. 34

4.2.3. Desplazamientos hacia atrás. 35

4.2.4. Desplazamientos hacia arriba o saltos. 36

4.3. Relación entre el bote de la pelota y los tipos de cancha. 40

4.4. Análisis de las competiciones en el tenis. 43

4.4.1. Análisis de competiciones en diferentes tipos de cancha o pista. 45

V. RESULTADOS DE LOS ANÁLISIS DE LAS COMPETICIONES. 108

5.1. Tipos de desplazamientos más utilizados en las competencias. 108

5.2. Distancias recorridas en la competición. 111

5.2.1. Distancias cortas más recorridas. 111

5.2.2. Distancias largas más recorridas. 112

5.3. Tiempos verdaderos de trabajo pausa en la competición. 112

5.4. Promedios y relación de utilización de los tipos de golpes en el "peloteo previo". 114

VI. ACONDICIONAMIENTO FÍSICO PRECOMPETITIVO PARA EL TENIS.

6.1. Acondicionamiento Físico Precompetitivo para el Tenis. 116

6.1.1. Especificidad en los tipos desplazamientos y sus distancias para el Acondicionamiento Físico Precompetitivo

6.1.2. Especificidad en tiempos de trabajo-pausa para el Acondicionamiento Físico Precompetitivo.

6.1.3. Aspectos metodológicos a tener en cuenta para una realización de Acondicionamiento Físico Precompetitivo.

VII. Bibliografía. 


\section{DEDICATORIA}

Esta tesis está dedicada mi mamá Zoraida Pinilla Rubiano, mi hermana Claudia Milena Rivera Pinilla, mi hermano Edwin Alexander Rivera Pinilla y a toda mi familia que creyó en mí y en mis posibilidades. 


\section{AGRADECIMIENTOS.}

Agradezco todo el apoyo y buena camaradería que me ha brindado la gente de La Plata y La Argentina. A la Universidad Nacional de La Plata y su facultad de Humanidades por darme la oportunidad de seguir aprendiendo cada día, a los honorables profesores por darme una guía y permitirme ser como estudiante. Agradezco también especialmente a Paula Peñalva, mamá de mi hija por su apoyo lector en esta tesis. 


\section{INTRODUCCIÓN.}

Las investigaciones en el deporte se hacen válidas en el sentido de su aporte al mejoramiento, desde el aspecto técnico, psicológico o físico, para potenciar las capacidades propias de los deportistas y mejorar el rendimiento en un evento deportivo. Es así, que también las nuevas perspectivas que sobresalen alrededor de las recientes investigaciones sobre el deporte, proporcionan un espectro más amplio y renovador para la aplicación, en pro de la consecución de más y mejores marcas, de mayor rendimiento y menor desgaste, de más eficacia y eficiencia, de más y mejores resultados. La "especificidad deportiva" será importante aquí porque nos aportará conocimientos y datos relevantes, al igual que propicio para la actividad deportiva que estudiaremos, la cual será, tenis de campo y, que en el trascurso de esta tesis será el derrotero a seguir para desarrollar nuestra propuesta de "Acondicionamiento Físico pre Competitivo para el tenis".

Vamos a decir que nuestra tesis tiene en cuenta una línea de trabajo basado en la lectura de lo que hay acerca de la preparación del deportista para una competición tenística en el momento inmediatamente anterior a la competencia conocido como "calentamiento". Seguiremos luego acercándonos a lo que se ha escrito sobre "acondicionamiento físico" para tener una base de referencia y de sustento desde lo que refiere la palara. Luego vamos a estudiar lo que pasa en una competición tenística de acuerdo con: los tipos de desplazamientos, los momentos de trabajo-pausa, los tipos de superficie donde se juega y su incidencia para con el juego $\mathrm{y}$, por último, tomando como referencia lo investigado, intentaremos realizar una propuesta de cómo se debería realizar el "Acondicionamiento Físico Precompetitivo" para un jugador o jugadora de tenis en el que entre dispuesto al cien por ciento de sus capacidades físicas propias para la competición. 
En cuanto al "acondicionamiento físico", nos encontraremos con algunas lecturas sobre su historia desde el comienzo en el mundo deportivo y, cómo ha venido ayudando siendo parte de la preparación de los deportistas, definiendo un proceso de entrenamiento que tiende a mejorar el estado físico de una persona, o bien, mejorar el estado óptimo de rendimiento de acuerdo a las cualidades o capacidades físicas, igualmente utilizado en el mundo tenístico. También estaremos citando las descripciones teóricas consignadas por algunos estudiosos del tenis que hablan sobre el "calentamiento" y los tipos de calentamiento que se utilizan; los tipos de desplazamiento en el tenis y los tiempos de trabajo-pausa para tener una idea de lo que se sabe de este momento previo a la competición. No dejaremos de lado tampoco lo que debemos saber acerca de las reglas del deporte, de las dimensiones de la pista o cancha en que se juega y tampoco de sus clasificaciones según su velocidad de la superficie, pues estas referencias serán de suma importancia para tener en cuenta en nuestra investigación y posterior propuesta.

Uno de los momentos más interesantes de nuestra tesis, será , el desarrollo de las observación minuciosa en cuanto a: los tipos de desplazamientos en el tenis, sus frecuencias de usos, sus distancias de recorridos, sus porcentajes y promedios de utilización, los momentos de trabajo-pausa globales y específico punto por punto y, la altura de los impactos de la pelota de acuerdo a cada tipo de cancha; todo esto, en partidos de hombres y de mujeres en diferentes torneos de más alto nivel (Grand Slam, Master Mil y ATP 250). A partir de ello, obtendremos una plataforma informativa que nos arrojará datos importantes a tener en cuenta para poder diagramar conclusiones importantes y específicas del tenis en competencia y, así, generar nuestra propia propuesta de cómo preparar a un deportista desde el "Acondicionamiento Físico Precompetitivo" para un partido de tenis, la cual puede ser tomada o no como guía o referente a tener en cuenta. 


\subsection{Tema.}

El tema a desarrollar en nuestra tesis será el "Acondicionamiento Físico Precompetitivo para el Tenis" y, este hace referencia a la preparación física del deportista minutos antes de ingresar a una competición tenística. De acuerdo con esto, teniendo en cuenta la recolección de datos y el estudio que en esta tesis se realizará; buscaremos relacionar lo que se ha propuesto desde la teoría en cuanto al "calentamiento" y, el estudio de la competencia teniendo como referencia las exigencias físicas propias del deporte; para luego obtener datos importantes y relevantes que sustentarán la construcción y valides de nuestra propuesta.

\subsection{Problemática.}

Se puede encontrar en la bibliografía, que para el tenis existen varias formas de prepararse físicamente minutos antes de la competencia. Estas formas, obedecen a teorías desde el deporte en general y desde el calentamiento precompetitivo del tenis. Lo que no se logra encontrar es una línea verdaderamente específica en la preparación o calentamiento del deportista antes de entrar a la competencia; me refiero aquí a conceptos claros y directos que apunten a aspectos como cuales deberían ser los porcentajes y frecuencias de los ejercicios que se deben trabajar en el calentamiento, de acuerdo a: los tipos de desplazamientos, momentos de trabajo y pausa para cada tipo de cancha, movimientos y ajustes corporales de acuerdo a los diferentes tipos de cancha $\mathrm{y}$, el tiempo de utilización del momento de preparación o calentamiento precompetitivo.

Es a partir de esta problemática, donde encontramos pocas herramientas teóricas que favorezcan la preparación específica del deportista en el calentamiento para cada tipo de cancha previo a la competición. Y es aquí, donde pensaremos en una propuesta integradora para la preparación del deportista, la cual surgirá también desde el estudio que haremos a los partidos de alto nivel competitivo en cuanto a las exigencias físicas. 


\subsection{Objetivo general.}

A partir de los referentes bibliográficos y las observaciones de competencias tenísticas de primer nivel, se pretende desarrollar una nueva propuesta específica para que un deportista, momentos antes de entrar a la competencia, ingrese en su mejor rendimiento físico posible.

\subsubsection{Objetivos específicos.}

- Analizar la bibliografía existente para estudiar lo que hay sobre el momento previo a la competición "calentamiento" y "acondicionamiento físico".

- Tener en cuenta estadísticamente los tipos de desplazamientos de acuerdo al porcentaje de utilización.

- Obtener estadística a partir de la observación de los momentos de trabajo y pausa en la competencia tenística.

- Analizar la altura del golpeo de la pelota en cada tipo de superficie.

- Detallar de acuerdo a la especificidad del deporte, cual o cuales son los sistemas energéticos utilizados por el deportista.

\subsection{Metodología.}

Para diagramar los pasos que tomará la tesis, es imprescindible saber en primera medida que nuestro eje temático es el "acondicionamiento Físico Precompetitivo para el Tenis", el cual está determinado por el alistamiento o preparación previa del deportista físicamente antes de comenzar un partido de tenis. Será claro aquí entonces que nuestra línea será el rendimiento deportivo, hallándose centrado en y solamente desde el aspecto físico específico para afrontar las exigencias que la competencia depare.

Es importante tener en cuenta la problemática que surge en el marco de nuestro eje temático, la cual nos lleva a la pregunta "¿Qué es lo que se sabe o lo que hay acerca de la preparación física del tenista minutos antes de la competencia? y para esto echaremos mano a algunas reseñas teóricas de 
diferentes autores que nos hablarán al respecto, en cuanto al calentamiento en general y específicamente del tenis, del aspecto fisiológico del deportista; así como también, del acondicionamiento físico general.

Seguiremos a diagramar unas tablas de referencia para la visualización de los tipos de desplazamiento y sus distancias hipotéticas de recorrido que pueden suceder eventualmente dentro de una chacha de tenis y, que serán pieza clave de nuestro estudio. Donde también, servirán como soporte para otras tablas de marcación en cuanto a las observaciones propias que haremos de las competencias de algunos partidos de tenis de alto nivel referente a: distancias de cada tipo de desplazamiento realizado y su porcentaje de utilización, tiempo de trabajo-pausa de cada punto y sus porcentajes de utilización, distancias recorridas por punto y su porcentaje de utilización; todos estos para cada tipo de superficie donde se juegue.

Se tendrá en cuenta también mediante tablas de observación, los tiempos de utilización de cada tipo de golpe en el "peloteo previo" que se hace dentro de la cancha cuando se inicia la presentación del palmarés de cada uno de los jugadores momentos antes de dar inicio al partido.

Por último, a partir de estas observaciones, sacar conclusiones estadísticas válidas de acuerdo a las exigencias físicas del deportista en los diferentes tipos de cancha y, poder diagramar nuestra propuesta de "Acondicionamiento físico precompetitivo" que atienda a los requerimientos físicos propios del deportista en la competencia. 


\section{ESTADO DE LA CUESTIÓN}

Los referentes que vamos a mostrar aquí, hacen parte de los trabajos que se han realizado en el tenis para manejar el momento anterior a la competencia "calentamiento". Así como también se retomará aspectos importantes acerca del "acondicionamiento físico en el deporte", pues son los dos referentes importantes que en primera línea hablan de forma teórica como preparar a un deportista.

En el tenis de hoy en día, el tema de cómo entrar físicamente preparado a la cancha para rendir al máximo en un partido, está sesgado por el nombre de "calentamiento", pero muchas veces, los entrenadores, preparadores físicos y hasta los mismos deportistas carecen de las herramientas apropiadas para realizar dicho calentamiento. En muchas ocasiones los deportistas no hacen el calentamiento y, si lo hacen, es atendiendo muchas veces a un simple aumento de la temperatura corporal creyéndose que ya se está listo para la competencia.

El calentamiento cumpliría entonces su papel fundamental como lo dice la palabra, calentar o elevar la temperatura corporal. Ese calentamiento que le ayudará a jugar bien o mal, pero que logre elevar la temperatura corporal sería lo esencial, pues en teoría, las funciones orgánicas se desempeñan mucho mejor; pero, ¿qué se tiene en cuenta para realizar estos calentamientos antes de entrar a un partido?, pues con certeza, encontramos que en muchos deportes lo que se hace para elevar la temperatura corporal, es hacer estiramientos generales, hacer una carrera a intensidad media de unos 10 a 15 minutos de duración, hacer otros estiramientos y con esto culminaría la etapa del calentamiento para entrar a competir. Esto pues, es lo que se ha tenido en cuenta normalmente y tradicionalmente en el tenis.

Con lo anterior, tendremos que remitirnos al siguiente subtítulo, el cual nos comentará más afondo, cómo se ha venido tomando el calentamiento en el 
deporte, y cómo es que tradicionalmente se lo toma de bisagra principal que conecta la inactividad del deportista con la competencia. Crucial momento en el que el deportista debe poner a punto sus capacidades físicas para afrontar el partido.

\subsection{El calentamiento.}

En el deporte, como en la preparación física especializada, el tema envolvente de la -preparación previa- al esfuerzo deportivo o para cualquier actividad física, es tomada como "Calentamiento"; esto refiere a un alistamiento físico metabólico y mecánico en su gran mayoría si revisamos la bibliografía. Como bien lo comenta Blázquez, Sánchez Domingo (2004. 5), el calentamiento se realiza en la parte inicial de cualquier sesión, sea ésta educación física escolar, actividades de mantenimiento, sesiones de entrenamiento, o bien una competición.

Si bien, siguiendo la línea de Sánchez, Domingo (2004.5), no es sino hasta la llegada de lo que hoy conocemos como "educación física" cuando se propone de forma sistematizada que la primera parte de la sesión, se destine a esta función como entrada en calor. Por el mismo lado, Tudor O, Bompa (2004. 66), el propósito específico del calentamiento es preparar a los deportistas para el programa que han de seguir. Durante el calentamiento aumenta la temperatura corporal, lo cual parece ser uno de los factores principales que mejora el rendimiento.

Con lo anterior, Vladimir, N Platonov, (2001. 462), sugiere, que el calentamiento debe tender a tres objetivos siguientes: funcional, motor $y$ emocional. Apoyando este argumento, Jügen, Weinek, (2005. 575), "entendemos todas las medidas que antes de una carga deportiva, ya sea el entrenamiento o la competición, sirven para crear un estado de preparación óptimo en términos psicofísicos, coordinativos-cinestésicos, y para prevenir las lesiones. 
Tenemos entonces que en la bibliografía se hallan posturas teóricas que apuntan a un mismo lado, sin embargo, uno más general que otro y uno más específico que otro, por su lado, también, Verkhoshansky, L V (2000. 199) nos propone que: "el calentamiento sirve para que el cuerpo se halle en disposición para desarrollar cierta capacidad de trabajo"; y que también, citando a (Ozolín, 1944; Krestovnikov 1951; Miller, 1951; Blank 1955), la idea de tener dos tipos de calentamiento, un tipo de calentamiento general y un calentamiento específico; donde el general apunta a aumentar el potencial funcional del cuerpo, y el específico hace referencia a establecer una relación optima entre el ejercicio próximo y las actividades del sistema nervioso central.

Como vemos hasta aquí, los postulados que hay sobre el calentamiento, no es más que una buena organización de acciones físicas que permita al deportista desde un plano general, aumentar la temperatura corporal para mejorar las respuestas fisiológicas y, específicas como el alistamiento motor y emocional del deportista para un rendimiento óptimo como también para prevenir lesiones.

\subsection{Estiramiento en el calentamiento:}

La disminución de la producción de potencia es un posible resultado negativo del estiramiento, y esto debemos tenerlo en cuenta, pues en el tenis si o si hay muchos momentos de potencia; en el que la rapidez de contracción muscular está condicionada por la especificidad del deporte. Algunos estudios han demostrado una disminución de potencia inmediatamente tras el estiramiento. Kokkonen et al. (1998), "observaron que el estiramiento estático antes de un partido disminuye significativamente la repetición máxima $(\mathrm{MR})$ tanto en la flexión como en la extensión de la rodilla".

La producción de potencia es la función de desarrollar rápidamente tensión en los músculos. Hay dos problemas potenciales que pueden ocurrir al estirar estáticamente un músculo antes de una actividad balística: la disminución en la producción de potencia debido a, en primer lugar, la laxitud del músculo, y en segundo lugar, a un descenso en el reclutamiento a nivel del huso 
neuromuscular; por tanto, es posible que un jugador sufra una disminución en la producción de potencia inmediatamente tras una sesión de estiramientos extensivos antes de un partido.

\subsection{Acondicionamiento físico.}

En el mundo del deporte y la actividad física en general, se ha utilizado el término "Acondicionamiento Físico", para definir un proceso de entrenamiento que tiende a mejorar el estado físico de una persona, o bien mejorar el estado óptimo de rendimiento de acuerdo a las cualidades o capacidades físicas. Este término ha sido acuñado en los años 80 desde la fundación en Estados Unidos de la A.F.A.A (Aerobic and Fitness Asociation of America), para definir un nuevo tipo de ejercicios para la salud y la belleza corporal, al que llamaban "fitness" y, al pasarlo a las otras lenguas, y en especial a la lengua española, se lo definió como "Acondicionamiento Físico". A esto, la Real Academia Española (RAE), nos dice que significa: "dar cierta condición o calidad" como también "Disponer o preparar algo de manera adecuada a determinado fin" y en el lenguaje venezolano, la Real Academia Española le da el significado de "entrenar a un deportista".

De este término que plantea la RAE, "dar cierta condición”, tenemos que saber también que en el mundo del deporte se utiliza como "condición física" y, si vemos al deportista como poseedor de un cúmulo de condiciones biológicas, en la que le es posible desarrollar ciertas actividades físico-deportivas; entonces este criterio sirve para tener en cuenta estas capacidades y ser mejoradas por medio del trabajo físico. En el diccionario de las ciencias del deporte (unisport.1992), podemos ver al respecto, "factor de la capacidad de trabajo (físico) del hombre, determinado por el grado de desarrollo de las cualidades motrices de resistencia, de fuerza, de velocidad y de flexibilidad", y, por otro lado, tenemos también algunas de las definiciones que encontramos al respecto en cuanto a "condición física", algunos la han definido como: Véase Tabla 1. 


\begin{tabular}{|c|c|}
\hline AUTOR & DEFINICIÓN DE CONDICIÓN FÍSICA \\
\hline $\begin{array}{l}\text { Barbany } \\
(1986)\end{array}$ & $\begin{array}{l}\text { Conjuntos de cualidades o capacidades motrices del sujeto, } \\
\text { susceptibles de mejora por medio del trabajo físico. }\end{array}$ \\
\hline $\begin{array}{l}\text { Grosser } \\
(1988)\end{array}$ & $\begin{array}{l}\text { Suma ponderada de las cualidades motrices (corporales) } \\
\text { importantes para el rendimiento y su realización a través de los } \\
\text { atributos de la personalidad. }\end{array}$ \\
\hline $\begin{array}{l}\text { De la Reina } \\
(2003)\end{array}$ & $\begin{array}{l}\text { Conjunto de cualidades o condiciones orgánicas, anatómicas y } \\
\text { fisiológicas, que debe reunir una persona para poder realizar } \\
\text { esfuerzos físicos tanto en el trabajo como en los ejercicios } \\
\text { musculares y deportivos. }\end{array}$ \\
\hline OMS (1992) & El bienestar integral corporal, mental y social. \\
\hline $\begin{array}{l}\text { Navarro } \\
(1990)\end{array}$ & $\begin{array}{l}\text { Parte de la condición total del ser humano que comprende } \\
\text { muchos factores, cada uno de los cuales es específico de su } \\
\text { materia. }\end{array}$ \\
\hline $\begin{array}{l}\text { Manno } \\
(1991)\end{array}$ & $\begin{array}{l}\text { Condiciones motores de tipo endógeno que permiten la } \\
\text { formación de habilidades motoras, son un conjunto de } \\
\text { predisposiciones o potencialidades motrices fundamentales en } \\
\text { el hombre que hacen posible el desarrollo de las habilidades } \\
\text { motoras aprendidas. }\end{array}$ \\
\hline $\begin{array}{l}\text { Wilmore } \\
\text { (1990) }\end{array}$ & $\begin{array}{l}\text { Capacidad o habilidad para realizar de moderados a vigorosos } \\
\text { niveles de actividad física, sin excesiva fatiga, y la capacidad de } \\
\text { mantener parecida capacidad durante toda la vida. }\end{array}$ \\
\hline
\end{tabular}

Tabla 1. Tomado de: El acondicionamiento físico orientado a la salud en la ESO, Escrito por Francisco Javier Jiménez Melero. (2008.16)

El acondicionamiento físico entonces, en primera medida, es una acción que consiste en lograr cierta condición o estado a partir de una determinada disposición del organismo, Generelo y Lapetra (1993), citados por Fernández, Jesús (2009. 9); definen acondicionamiento físico como "el desarrollo intencionado de las cualidades o capacidades físicas; el resultado obtenido será el grado de condición física"; al igual que, Hegedus, j (1977), define el 
acondicionamiento físico como "el estudio y el entrenamiento sistemático de las cualidades físicas". Pero tiempo atrás, en 1969 la NSCA (National Strength and Conditioning Association), que en español significa (Asociación Nacional de Fuerza y Acondicionamiento), el jefe entrenador de futbol y director de deportes de la universidad de Nebraska, marcaron una nueva tendencia en el mundo de los deportes universitarios, pues fue el primer director de deportes en contratar un grupo de preparadores físicos, logrando triunfos y reconocimientos, que después llevaría a la primera convención de la NSCA en 1978.

La NSCA, marca un hito grande en lo que a la preparación física se refiere, pues desde allí se comenzó a orquestar un punto de partida para la especialización del entrenamiento en cada deporte y mejorar el rendimiento deportivo. Fue así que, tras la conformación de este grupo, el cual hoy es un gran referente desde la preparación física en Estados Unidos, comenzaron a ganar los primeros lugares de las competencias universitarias; y hacen de esta organización, una empresa que hoy por hoy es, si no, la más grande a nivel de preparación de entrenadores de acondicionamiento físico y entrenamiento personalizado.

Con todo lo anterior, tenemos que, la NCSA toma el tema del "acondicionamiento físico", como pilar para potenciar el rendimiento físico y deportivo, es así que, desde sus comienzos hasta hoy, la NCSA se ha encargado en todo Estados Unidos de preparar los mejores deportistas de diferentes deportes a nivel universitario.

Algo similar nos propone, Foran, Bill (2007. 7), los deportistas realizan actividades de acondicionamiento físico diseñadas fundamentalmente con la finalidad de mejorar su propio rendimiento en el deporte. La intensidad, duración y frecuencia de los esfuerzos aeróbicos y anaeróbicos se diseñan según los requerimientos de resistencia y potencia propios de la actividad, Bompa, T citado por, Foran, Bill (2007. 7), argumenta que ha desarrollado 
nuevos principios del entrenamiento que ha permitido que el proceso de acondicionamiento sea más sistemático y productivo; a esto, como lo sigue diciendo Bill, la intensidad, duración y frecuencia de los esfuerzos aeróbicos y anaeróbicos, se diseñan según los requerimientos de la actividad.

Para seguir argumentando y sustentando la importancia de un acondicionamiento físico, en palabras de Campos, José y Cervera, Víctor (2003), proponen que: los principios metodológicos del acondicionamiento físico, debe atenerse a una serie de principios generales que hay que considerar cuando se diseña cualquier plan de entrenamiento dirigido a la mejora de la capacidad funcional de los deportistas. Estos principios a lo que se refiere "Campos y Cervera", hacen referencia a que dichos principios establecen las condiciones básicas a partir de las cuales se logra la adaptación del organismo a las situaciones de estrés que conlleva la práctica deportiva y, como consecuencia última, abren la posibilidad a la mejora del rendimiento motor y funcional.

De hecho, estos principios metodológicos han establecido las claves fundamentales para el establecimiento de las diferentes propuestas teóricas que a partir de los años cincuenta, han revolucionado el contexto de la planificación del entrenamiento. (Letunov, 1950; Matveiev, 1956; Fidelus, 1960; Arosiev, 1971; Verjoshansky, 1978; Bondartchuk, 1984). Cada deporte requiere una metodología diferente, sin embargo, en lo que concierne al aprendizaje de la técnica, a la mejora de la capacidad física o a la optimización de los recursos tácticos estratégicos, los principios que rigen el proceso general del Acondicionamiento Físico constituyen normas de uso común. De todos ellos quisiéramos destacar los siguientes: principios de la unidad funcional, principio de la continuidad, principio del crecimiento paulatino del esfuerzo, principio de la especificidad, principio de la transferencia y principio de la individualización. 


\section{EL TENIS, SUS CARACTERÍSTICAS Y COMO SE HA TOMADO EL ACONDICIONAMIENTO FÍSICO Y EL CALENTAMIENTO EN ESTE DEPORTE.}

\subsection{La cancha de tenis y sus herramientas de juego.}

Para hablar de tenis, deberíamos tener conocimiento también de las, herramientas de juego que hacen posible que este deporte se realice. Estas herramientas son: la raqueta, la pelota y la cancha con su red divisoria. Lo importante de conocer estas herramientas es su grado de incidencia para con el juego de tenis; y aunque no nos vamos a centrar en ellas en toda la tesis, si las vamos a nombrar para saber por ejemplo, todas sus características que podrían aportar información valiosa, como que las raquetas ahora despiden la pelota mucho más rápido que antes, que hay diferentes tipos de pelotas para diferentes tipos de canchas, que el pique de la pelota varía en su altura según el tipo de cancha y,bien, saber las medidas de la cancha, pues de ella depende las distancias de los desplazamientos que realiza en deportista. Diferencias que dentro del mismo deporte son precisas para tener en cuenta en su práctica y estudio.

\subsubsection{La cancha de tenis y su red.}

Según la "ITF Regles Du Tennis 2014", La pista o cancha será un rectángulo de 23,77m (78 pies) de largo por 8,23m (27pies) de ancho. Para los partidos de dobles la pista será de 10,97m (36 pies) de ancho. A todo esto, el tenis también se practica en un terreno llano y puede ser: de césped o hierba, cemento y polvo de ladrillo, sin baches, sin desniveles, rectangular y siempre demarcado por unas líneas blancas de $3 \mathrm{~cm}$ de ancho menos la de fondo que puede ser de 5 a $10 \mathrm{~cm}$ de ancho.

La cancha de tenis está dividida por una red intermedia de $1,07 \mathrm{~m}$ de alta en los postes que la sostienen a los lados de las líneas de sencillos o de dobles, pero 
en el centro la altura es de $0,914 \mathrm{~m}$, esta red tiene una banda elástica blanca $5 \mathrm{~cm}$ de ancho sobre ella para ser observada por los jugadores y tomar como referencia su altura.

Vamos a tener en cuenta también, un lenguaje del tenis para comprender de qué estaremos hablando más adelante en los siguientes capítulos. La "T" (figura que se forma en forma de "T" por la intersección de la línea de servicio y la línea divisoria de los cuadros de servicio), la "línea media de fondo" (es la intersección de una pequeña línea de $10 \mathrm{~cm}$ de largo con la línea de fondo), línea de sencillos (es la línea que delimita a los lados el cuadro de juego de dos jugadores), línea de dobles (es la línea lateral que delimita el cuadro de juego de 4 jugadores), y, entre otras cosas, tenemos todas las demás medidas de la cancha para tener en cuenta, pues son las medidas estándar de una cancha de tenis, sea la superficie que sea. Véase imagen1.

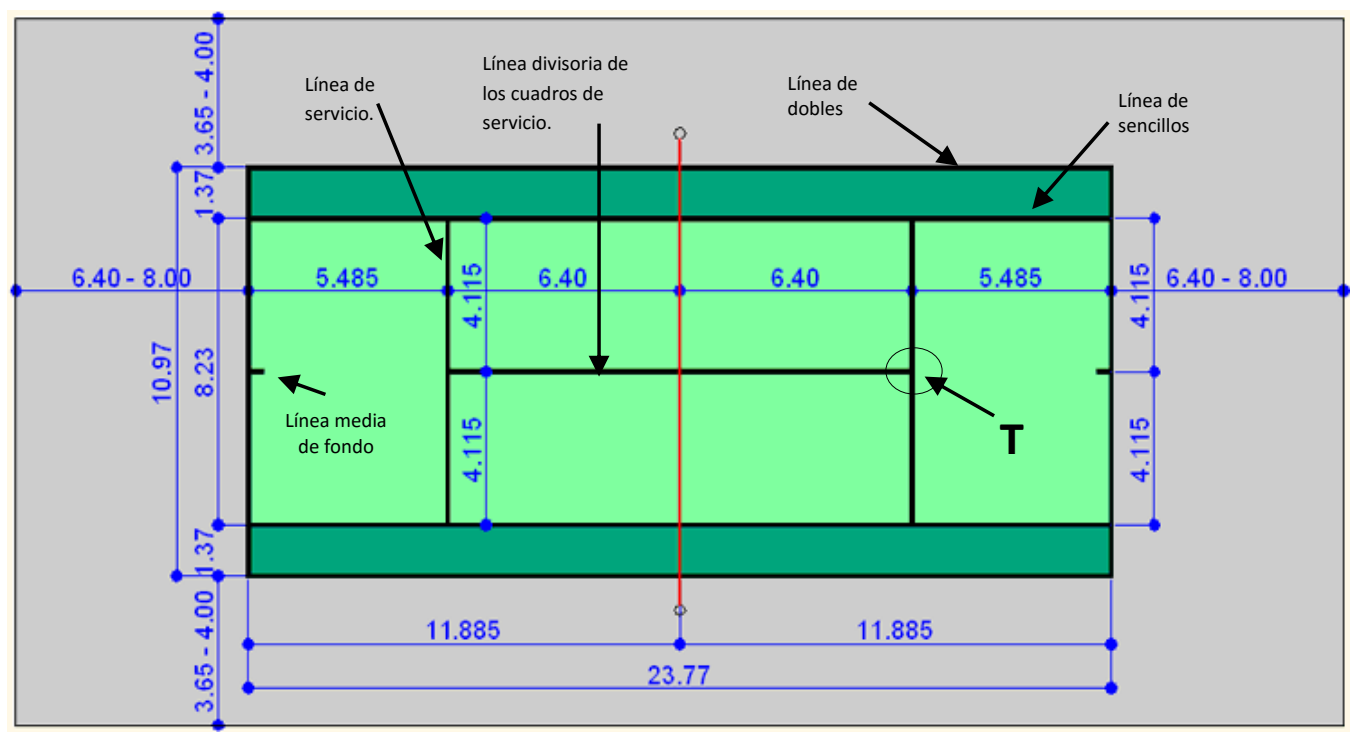

Imagen 1. Gráfico de cancha estándar de tenis.

\subsubsection{La raqueta.}

En la actualidad las raquetas son fabricadas con aleación de grafito y fibra de carbono, pues son las que proveen de mejores prestaciones a la hora de jugar y además de esto son resistentes y delgadas para tener menos resistencia con 
el viento, permitiendo una sensación favorable a la hora de golpear la pelota. Sus pesos para competición oscilan entre $270 \mathrm{gr}$ y $345 \mathrm{gr}$, aro entre $600 \mathrm{~cm}^{2}$ y $700 \mathrm{~cm}^{2}$, un largo de $68 \mathrm{~cm}$ y $69,5 \mathrm{~cm}$, un balance entre $305 \mathrm{~mm}$ y $325 \mathrm{~mm}$.

Cada jugador o jugadora elige una a su gusto y acomodo, de acuerdo a su sensación, pues es un tema muy subjetivo y de conocimiento empírico cual es el tipo de raqueta que más conviene para que no presente molestias a la hora de jugar. Claro está que algunas raquetas traen unas especificaciones para los tipos de ejecuciones, potencia y control de la pelota a la hora de impactar. El jugador y entrenador también se percata, generalmente de la elección del peso de la raqueta para no generar con el uso, alguna tendinitis o desgarros musculares por su excesivo peso o viceversa.

Hay raquetas que despiden más rápido la pelota, haciendo que el tiempo de vuelo de la pelota sea más corto, pero requiere más ajuste y control por parte del ejecutante y el tiempo de reacción para el oponente tenga que ser más rápido en sus desplazamientos, ajustes e impactos. También hay raquetas que despiden menos pero que son más fáciles de controlar los golpeos y hay que imprimirle más potencia a la hora del golpeo. Ambas son combinaciones existentes dentro del tenis que sin lugar a dudas hacen que el tenis sea cada vez más exigente y, por ende, el jugador debe estar cada vez más preparado.

\subsubsection{La pelota de tenis.}

Esta es una esfera de caucho con aire comprimido por dentro y recubierta de felpa color blanco o amarillo; el peso de la pelota ronda los 56 a 59,4 gramos y tiene un bote estándar de $135 \mathrm{~cm}$ a $147 \mathrm{~cm}$. Hay también felpas de la pelota para canchas de polvo de ladrillo, para canchas duras y para canchas de hierba. Los botes de la pelota en el juego varían mucho, dependiendo el tipo de golpe y efecto y; el jugador deberá sortear de la mejor manera y a veces exigiéndose al máximo para lograr sus ejecuciones.

En el siguiente capítulo, veremos cómo incide el bote de la pelota en los diferentes tipos de cancha, ya que, en algunas canchas, el bote de la pelota 
será bajo, en otras el bote será medio y en otras será alto; si bien se hablará también que la superficie de la cancha tiene que ver con la velocidad de la pelota y su altura, la pelota, también aporta su cuota como factor a tener en cuenta.

\subsection{El calentamiento y su tradición en el tenis.}

Tanto en otros deportes como en el tenis, el "calentamiento" se investiga, se escribe y se aplica nuevas o viejas formas de entrenar, pero, sobre todo se siguen algunas líneas tradicionales y poco apropiadas para la preparación del deportista previo a la competición como se ha visto en la práctica. A todo esto, vemos, por ejemplo, referentes que hablan del tenis, Le Deuff, Hervé (2005. 51), el cual, propone que, "el calentamiento tradicional antes del partido o del entrenamiento se inicia con un footing suave seguido por ejercicios de estiramiento y de movilidad articular, para luego, realizar ejercicios de reforzamiento y acabar con ejercicios de velocidad.

Con lo anterior, podemos ver, Le Deuff (2005. 51), sostiene también que, "el calentamiento común solo ayuda a calentar los músculos superficiales y que por eso propone un tipo de calentamiento para elevar la temperatura de los músculos más profundos, exponiendo la alternancia de ejercicios de fuerza y de estiramiento, los cuales, permitirían calentar el conjunto muscular progresivamente con gran eficacia".

Por otro lado, también en relación con el tenis, encontramos en la bibliografía, un autor que pone de manifiesto algunas consideraciones sobre el calentamiento en el tenis y lo que se debe hacer antes de una competencia, nombrando cinco momentos, Ortiz, Rendey (2004. 64), empezando por, 1) el calentamiento general (trote suave, movilidad, flexibilidad y peloteo), 2) calentamiento específico técnico (rapidez gestual de golpeo), 3) calentamiento específico técnico (ejercicios de reacción), 4) calentamiento específico técnico (ejercicios de velocidad de desplazamiento y movilidad en los pies), y, por último, 5) la recuperación (hidratación y elongación)”. 
Al parecer, este es uno de los trabajos que hablan de forma más específica y metodológica sobre cómo se debe hacer un calentamiento para un partido, terminando con un momento el cual llama "recuperación" y dice que no debe haber mucho tiempo entre este último y el partido, porque perdería todo sentido y validez el calentamiento.

Algo en contraposición con uno de los puntos anteriores; encontramos que, Alvares, Javier (2011. 3), subraya en su revista de multimedia, que, "en el tenis, la forma más común pero la menos efectiva para calentar es pelotear. Aunque este peloteo es una forma de prepararse para la sesión o el partido posterior, los jugadores que usan este método tienen tendencia a no ejercitar todos sus músculos hasta que comiencen los movimientos extremos del juego real y el no calentar bien puede provocar lesiones"; a esto, también se propone que calentamiento se pueda dividir en dos fases: un trabajo físico suave y un trabajo de estiramientos.

\subsubsection{El estiramiento en el calentamiento de tenis.}

El estiramiento es un factor fundamental en la vida deportiva. Los componentes de flexibilidad y elasticidad, son componentes musculo-esqueléticos muy importantes para la movilidad del cuerpo debiendo estar dispuestos de la mejor forma antes, durante o después del calentamiento según como lo tome cada entrenador o estudioso del tema. Tenemos entonces, que, Le Deuff, Hervé (2005. 18). "la flexibilidad permite realizar movimientos en su máxima amplitud; depende de la elasticidad muscular y ligamentaria".

El estiramiento en el calentamiento no ha sido tan descrito técnicamente hablando en el tenis, pero si se habla del estiramiento de forma general vinculado a propiedades globales del sistema muscular, articular y tendinoso, Vila, Carlos (2006.115), dice que el estiramiento, "es una de las capacidades condicionadas para desarrollar el movimiento y la amplitud de una articulación". Encontramos también que La Asociación Americana de Tenis (USTA) por 
medio de, Paul, Roetert y Todd, Ellembeker (2000. 42), presenta la siguiente tabla en la cual hay un foco importante en el estiramiento. Ver tabla 2.

\section{Aplicación de los estiramiento en el tenis}

1. Calentamiento general (de tres a cinco minutos) para aumentar la temperatura de los tejidos (joggin lento. Saltos, etc.).

2. Estiramiento estático del muslo y de zonas delimitadas.

3. Estiramiento dinámico con aumentos progresivos del rango y la velocidad de los movimientos.

4. Jugar al tenis.

5. Estiramiento estático después del ejercicio para evitar el dolor muscular y ganar flexibilidad.

Tabla 2. Tomado de, La Asociación Americana de Tenis (USTA) por medio de Paul, Roetert y Todd, Ellembeker (2000. 42)

Si observamos la tabla anterior, y hacemos un pequeño análisis del estiramiento en el momento del calentamiento o el entrenamiento, vemos que se lo ha tomado como parte constitutiva de toda una sesión y que siempre está después de un trabajo físico y, justo antes de jugar al tenis; ¿esto por qué?, podríamos encontrar algunas respuestas, pero de eso no nos ocuparemos en esta tesis más que, dedicar unos apartes y proposiciones de cómo se debería usar el estiramiento y en qué momento previo a la competencia (partido de tenis).

\subsection{El acondicionamiento físico en el tenis.}

Esta línea de pensamiento que se ha venido desarrollado en la mayoría de deportes, y como habíamos visto en el capítulo anterior a cerca del surgimiento y aplicación del acondicionamiento físico en Estados Unidos, entendimos, que este enfoque atiende a la preparación del deportista en su dimensión física; proveyéndolo de todas sus capacidades condicionales y coordinativas generales propias del deporte que se practique. Esto quiere decir que se preparara físicamente a un deportista para que rinda de la mejor manera en su deporte 
El acondicionamiento físico también ha sido utilizado como un desarrollo de cualidades físicas básicas, mantención de la musculatura, y factores biomecánicos del deportista; como también, Vila, Carlos (2006. 33) "el acondicionamiento busca un desarrollo físico multilateral, para favorecer a un mediano plazo una mejora de las capacidades específicas que intervienen en la preparación física especial". Tenemos entonces que este desarrollo físico multilateral aquí, no tendría en cuenta las formas técnicas de cada deporte, ni sus tipos de desplazamientos, ejecuciones de movimientos propios de cada disciplina y hasta su trabajo en el propio campo de juego; pues de eso se encargaría la "preparación física especial", es decir, habría que destinar otro espacio más para la preparación del deportista.

Con lo anterior, quisiera resaltar que si bien, se encuentra dos posturas que apuntan a la preparación del deportista, el "acondicionamiento físico y la preparación física especial". Posteriormente, en nuestra tesis, vamos a tener un solo enfoque que englobe de manera pertinente lo específico del tenis $\mathrm{y}$, que el acondicionar físicamente a un jugador de tenis, sea, prepararlo desde las capacidades físicas y técnicas propias de este deporte para rendir de la mejor manera en un partido. Esto, teniendo en cuenta todo lo que a partir de la investigación hicimos y que explicaremos en el siguiente capítulo para luego proponer lo que se debería realizar antes de entrar a una competencia de tenis.

\subsection{Perfil fisiológico del tenista.}

Desde la programación del ejercicio, se debe tener en cuenta cuál es el perfil fisiológico que se está atendiendo en el deportista; tal es así que, si no tenemos la más mínima idea de lo anterior, incurriremos en especulaciones someras y tradiciones de lo que se ha trabajado para "alcanzar" el buen rendimiento del deportista. Por tal motivo es indispensable saber sobre el deporte en el que el deportista se desarrolla, y de ahí, conocer los tiempos en que se ejecuta cada trabajo, cada descanso, las intensidades y frecuencias de esos trabajos y descansos. Conociendo esto, entraríamos ya en el terreno de lo 
específico del deporte y sobre ello comenzaríamos a comprender también cómo es que funciona el cuerpo "desde lo fisiológico".

Ahora bien, para el desarrollo de los sistemas energéticos específicos de este deporte, el entrenador, debe saber y comprender estos datos que desde la ciencia vienen estudiados, para así poder programar un buen entrenamiento al deportista que preparará para las futuras competencias. Estos entrenamientos deben ir dirigidos al desarrollo de esas vías metabólicas o vías energéticas y aquellos patrones de movimientos que predominan en la competición, tratando de respetar en la medida de lo posible, el tipo de intermitencia que se presenta entre los tiempos de trabajo y los tiempos de pausa, como también los grupos musculares que se utilizar en dichas ejecuciones de movimientos (como golpes, carreras, saltos, derrapes y posiciones).

Una de las principales características de éste deporte, es su calidad de intermitencia, su particularidad en tiempos de desarrollo y en su forma de requerir a las exigencias metabólicas haciendo que los tenistas jueguen largas o cortas jornadas, siempre a la misma intensidad. De lo cual, Naclerio, F y Cols (2011. 324) "En estas circunstancias la mayor o menor implicación aeróbica y/o anaeróbica vendrá dada por la estructura temporal, fundamentalmente por la duración de los puntos y la ratio $W / R$, aunque también por la cantidad de masa muscular implicada y la calidad de trabajo muscular al golpear y desplazar".

En cuanto al perfil fisiológico de los y las jugadoras de tenis tienen que ver entonces como hemos dicho anteriormente, en relación a los tiempos de trabajo-pausa en el tenis; donde los trabajos son en un corto lapso de tiempo 1" a 9" en promedio, y los descansos de 15" a 25" en promedio; superando en dos, tres o cuatro veces y más, al tiempo de trabajo dependiendo cada punto, pues puede haber un punto que dure 1" y se descanse 19" o a la inversa, que se trabaje 35" y se descanse 30". Y para lograr que esto suceda, en la contracción muscular es necesaria la liberación de calcio $\left(\mathrm{Ca}^{2+}\right)$, y la participación de una molécula altamente cargada de energía, conocida como 
ATP, que es lo que el músculo tiene como mayor fuente de energía a su disposición, luego viene su re síntesis gracias al fósforo $(P)$ libre en la célula para seguir generando energía.

Ahora, si hablamos del tipo de fibras musculares para el trabajo mecánico, y adhiriéndonos como lo hemos venido diciendo, al tiempo de trabajo-pausa, encontraremos en nuestras investigaciones que los tiempos globales de trabajo y descanso, varían ente 1" de trabajo a 3,5" y 6" a 35" de descanso. Si bien se descansa más de lo que se juega, tendríamos que conocer también, que las ejecuciones de los movimientos se hacen a grandes intensidades y en cortos lapsos de tiempo, pero con un corto lapso de tiempo también para recuperar esas demandas energéticas que nos requirió tal esfuerzo. En conclusión, si estamos hablando de movimientos intensos y poca duración en el tiempo; estaremos hablando de los tipos de fibras de contracción rápidas o glucolíticas tipo II.

Estos tipos de fibras tipo II, estudiadas en el laboratorio, y comprobada su capacidad de contractibilidad rápida, Naclerio, F y cols (2011. 37), argumentan que "poseen isoformas MHC-IIA y MHC-IIX (estas últimas son las que tienen más actividad ATPasa) y el sistema de acoplamiento excitación-contracción está más desarrollado que en las fibras tipo I (túbulos trasversos, retículo sarcoplasmático, proteínas y encimas asociadas). Esto permite a las fibras tipo II un almacenamiento mayor de $\mathrm{Ca}^{2+}$, liberarse al sarcoplasma y recaptarlo más rápidamente. Por ese motivo la contracción y relajación de estas fibras es más rápida que las fibras tipo I".

Observamos que los tenistas mantienen una capacidad alta de esfuerzos intermitentes gracias al entrenamiento y por las características propias del deporte, hacen que su sistema de recuperación sea muy rápido; es decir, su sistema adaptativo específico al deporte está tan adaptado valga la redundancia, a estas exigencias, que siempre estará en condiciones de efectuar otro movimiento rápido, potente y con calidad neuromuscular a pesar 
de la fatiga central o periférica acumulada con el paso de las series. Es así que las preparaciones que el deportista debe hacer, apuntarán específicamente a estas referencias propias del deporte para alcanzar su máximo de rendimiento en el tenis.

Como se observa, en el tenis, se genera un movimiento rápido y explosivo al impactar una pelota, que será <1", entonces estaríamos hablando de la utilización altamente energética que proviene del ATP, Naclerio, F (2011. 4), "sin embargo, las reservas de ATP en la célula son muy escasas (5-10-6 mol.gr1 de músculo esquelético)". Ahora, si tenemos en cuenta también, que los desplazamientos son a gran velocidad con tiempos $>1$ " o 2" \pm 3 ", y utilizando lógicamente grandes grupos musculares de las extremidades inferiores, estaríamos hablando entonces que el sistema fosfagénico proporcionará la energía necesaria para estos movimientos breves y de elevada intensidad.

Ahora, si hablamos de tiempos globales, en los momentos de descanso el cuerpo tiene que recuperarse para el siguiente punto, pues bien, aquí entraría el sistema aeróbico en juego, donde, Naclerio, $\mathrm{F}$ y cols (2011. 324), "el VO 2 se mantiene elevado para recuperar el equilibrio homeostático a través de procesos como rellenar los depósitos de oxígeno celular, resintetizar la PCr, metabolizar el Lactato y eliminar el $\mathrm{Pi}$ acumulado. Esta dinámica de recuperación rápida permite considerar el esfuerzo intermitente como un continuo".

Con todo lo anterior, decimos entonces que el perfil fisiológico del tenista, es de carácter mixto, pues mientras funciona un sistema energético para la ejecución de los golpes y desplazamientos, actúa el otro para recuperar esos esfuerzos durante las pausas.

\subsection{Movilidad en el tenis.}

Este es un factor muy conocido en el mundo del tenis y que se escucha hablar sin parar en las sesiones de entrenamiento y comentarios de partidos, en los que al perdedor se le dice que a falta de movilidad perdió un partido y al 
ganador su destreza para moverse en la cancha lo hace superior y logra ganarlo. Es así que la movilidad se hace muy importante en este deporte, pues la pelota no siempre viaja por la mitad de la cancha, y hay que estar predispuesto y dispuesto para recorrer cortas distancias ,1m a 4m, cambiar de dirección y luego volver a recuperar la posición y esperar un nuevo envío del otro lado para impactar la pelota de forma ajustada, segura y con buena dirección y puntería. Aquí pues, podríamos decir que la movilidad es una "capacidad" interconectada con el factor coordinativo y condicional del jugador o la jugadora.

La movilidad para un jugador de tenis viene sujeta también a los tipos de superficie en la que se juega, es así que, si juega en una superficie de arcilla, tendrá que moverse más que en una cancha de césped o una de cemento; por el mismo lado, Kovacs, Mark $(2014,2)$, "estas superficies diferentes de cancha dan como resultado diferentes exigencias en el movimiento debido a la velocidad, amortiguación y fricción de la cancha". Por tal razón se hace importante tener en cuenta estos aspectos para la movilidad del tenista.

Las acciones controladas, ajustadas y a velocidad óptima; hacen que los deportistas se sitúen de la mejor manera para poder impactar la pelota que viene del otro lado. Como bien lo decía, Groppel, citado por Ortiz, Rodríguez Rendey (2004. 105), "el principal factor limitante del éxito de un jugador es el movimiento". Una buena movilidad coloca al jugador donde debe estar para impactar bien. Es así que un jugador se debe preparar para esto y no para otros requerimientos; debe alistar de la mejor manera sus respuestas neuromotoras para la rápida respuesta, coordinación de espacio-tiempo en el golpeo, fuerza y velocidad controlada para el desplazamiento, acomodación y relajación para una rápida recuperación o momento previo al golpeo, el cual es un momento en el que se observa muy baja tensión muscular para luego generar gran explosividad. 


\section{ESTUDIO DE LOS MOMENTOS Y ACCIONES DEL/A TENISTA EN EL LA COMPETICIÓN.}

El principal factor limitante del éxito de un jugador es el movimiento (...) Realmente no importa la calidad de unos golpes si no se está donde se debe estar (...) De hecho, el 70\% de los errores que comenten los jugadores de calidad, no son debidos a la mecánica del golpe, sino a la movilidad.

Groppel (1993, 59), citado por Ortiz, Rendey $(2004,105)$

\subsection{Desplazamiento del tenista en la competición.}

El tenis como otros deportes (futbol, el básquetbol, squash, balonmano, etc.), poseen un rico espectro de movimientos variados que posibilitan el buen desarrollo del juego. Para Kovacs, Mark (2014, 1), "mejorar el movimiento específico del tenis de un atleta requiere que el preparador físico o entrenador comprenda los patrones de movimiento que ocurren durante el juego". Encontramos que el desplazamiento es un tema fundamental en nuestro estudio, ya que es un determinante del deporte que vamos a investigar, y es una línea fuerte en todo lo que respecta nuestra tesis. Podremos observar entonces que hay desplazamientos en todas las direcciones, como también, ajustes, cambios de dirección y saltos. Estas características como las que acabamos de nombrar, hacen diferencia con algunos deportes, como el ciclismo y atletismo (carreras) entre otros.

Siguiendo con lo anterior, en relación al tenis, tenemos que hablar de una especificidad singular entre su mixture de capacidades, pues en este deporte tenemos unas características propias que lo hacen único como los demás, y por ese motivo hay que estudiarlo según sus propiedades. Es así que, si bien, tenemos una variedad de aspectos para estudiar en el tenis, la perspectiva más fuerte que iremos a desarrollar en esta tesis, hace referencia a "los tipos de 
desplazamientos", y a partir de ella generar una propuesta alternativa al tema de cómo pensamos el "Acondicionamiento Físico Precompetitivo para el tenis".

Si echamos un vistazo al respecto, un buen punto de vista bien específico al deporte del que estamos hablando, sería esta, "una actividad deportiva eminentemente técnica, pero con altas exigencias sobre los tres mecanismos que configuran la acción motriz. A esto, Naclerio, F y Cols (2011. 321), "las exigencias sobre el mecanismo de ejecución (máxima precisión, eventualmente a velocidades elevadas y en espacios reducidos), incluyendo también las variables de -estructura temporal del esfuerzo- exigencia condicional determinante y estructura temporal de la competición". Este sería entonces, un valor muy importante para tener en cuenta en su estudio y diferenciarlo respecto a las otras actividades deportivas.

De manera específica, pensamos que estas particularidades serían entonces, movimientos o desplazamientos, "a grandes velocidades, en espacios cortos, en lapsos cortos de tiempo y con un gran componente de ajuste corporal". Tenemos aquí entonces 4 elementos constitutivos del desplazamiento en el tenis; y, esto relacionado con los tipos de cancha, con las dimensiones de la cancha y el tipo de juego que ha venido evolucionando haciéndose más rápido, más fuerte y en definitiva más exigente; hacen que el estudio de los desplazamientos mientras se juega en una cancha de tenis, nos dé un sustento observable y medible para tener otra perspectiva 0 , romper un antiguo paradigma para postular otro acorde con la exigencias de dicho deporte en la modernidad.

Para tal motivo, y haciendo quizás un abrebocas de lo que se piensa en relación a los desplazamientos, las distancias y los tiempos; nos parece conveniente citar textualmente a Ortiz, Rendey, el cual nos muestra cómo en estudios realizado por diferentes investigadores, ponen de relieve una conclusión para las distancias que hipotéticamente se recorren en una cancha de tenis durante un partido. 
"Conforme a los estudios llevados a cabo por Elliot y cols. (1985), Schömborn (1987), Stojan (1987),Docherty (1982), entre otros, se ha llegado a la conclusión de que las distancias que se recorren en el tenis están entre $\pm 4 \mathrm{~m}$ por cada golpe, $\pm 14 \mathrm{~m}$ por punto, $\pm 85 \mathrm{~m}$ por juego, $\pm 1.100 \mathrm{~m}$ por set y $2.400 \mathrm{~m}$ o, inclusive mucho más por partido (para Roland Garros se implementó un dispositivo de tecnología altamente desarrollada con el fin de medir los desplazamientos de los jugadores, llegándose a encontrar recorridos entre 5.000 y 7.000 metros en el encuentro disputado entre André Agassi y Franco Squillari)". Ortiz, Rendey. (2004, 136).

Aunque estas distancias fueron descriptas así por los estudios que arrojan los resultados en promedio; de manera específica, estaría atendiendo de forma global dicha perspectiva de las distancias recorridas por un tenista. Pero entonces, no nos estaría dejando mucha claridad de que tipos de desplazamientos se realizan para recorrer todas estas distancias que el estudio arrojó. Para esto, haremos entonces en nuestra tesis, un análisis más minucioso para determinar cuáles son en realidad las distancias en promedio que se recorren en cada tipo de superficie, teniendo en cuenta, lógicamente las dimensiones reglamentarias de la cancha que desde las reglas de la ITF están propuestas.

Para desarrollar este tema hablaríamos, por ejemplo, de cuál es la mayor distancia que puede recorrer un tenista en un solo desplazamiento dentro de una cancha de tenis; realizamos entonces un cálculo matemático utilizando el Teorema Pitágoras y, tenemos que, la distancia más larga que puede recorrer un tenista es de unos $24,81 \mathrm{~m}$ promedio, (Ver imagen 2). Más adelante vamos a mostrar todas las posibles distancias recorridas dentro de una cancha de tenis, válidas para jugadores y jugadoras en cualquier tipo de superficie y en cualquier tipo de torneo. Estas distancias son siempre características de los recorridos que hacen los tenistas durante un partido. 


$$
\begin{aligned}
& D^{2}=L^{2}+L^{2} \\
& D^{2}=14,962 m t+19,8^{2} m t \\
& D^{2}=223,8 m t+392,04 m t \\
& D=\sqrt{615,84} m t \\
& D=24,81 m t .
\end{aligned}
$$

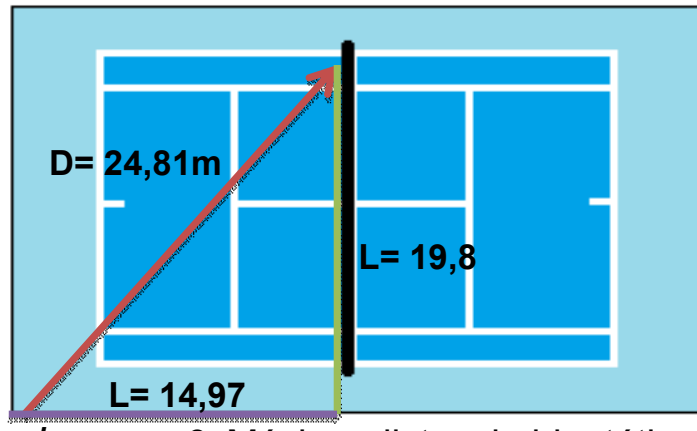

Imagen 2. Máxima distancia hipotética recorrida en un desplazamiento.

Entendemos entonces que, si bien hipotéticamente, se pueden recorrer $24,81 \mathrm{~m}$ en línea recta a máxima velocidad, parar e impactar la pelota, esta no es una acción que se presente comúnmente en un partido y, si se presenta, será una o dos veces a lo sumo en un torneo. Sí, por el contrario, volviendo a citar a Ortiz, R (2004.138), "en jugadas de peloteo continuo en cualquier superficie, los desplazamientos no son extensos $(2 m-8 m)$ pero si muy rápidos y repetitivos, dependiendo de la velocidad de la pelota, del ángulo que describa y del número de veces que se mantenga en juego". Con esto, y con lo que hemos venido diciendo acerca del tema de los desplazamientos en el tenis, quedaría más que lógico que en un partido un jugador o jugadora no va a recorrer siempre distancias mayores a 8 metros por ejemplo y, que teniendo en cuenta esto, un deportista para este deporte, no debería prepararse entonces para recorrer grandes distancias (100m. 200m, 500m, 1000m o más) como lo hacen en otros deportes.

Podemos ver también, un estudio que se hizo a las "distancias medias recorridas" en un partido hipotético, más los porcentajes de desplazamientos según la superficie "tierra o pista dura", (Ver tabla 3). Aunque nos muestra de manera más detallada los metros recorridos y sus porcentajes, los cuales serían válidos para tener en cuenta en cualquier propuesta de entrenamiento aplicativo más específico al deporte; en nuestra tesis tendrá temas que 
discutirán muy posiblemente con este y otros estudios que hacen referencia a los desplazamientos en el tenis.

\begin{tabular}{|l|c|c|}
\hline \multicolumn{2}{|c|}{ DISTANCIAS MEDIAS A RECORRER (Metros) } \\
\hline & Pista dura & Tierra batida \\
\hline Por golpe & $4 \mathrm{~m}$ & $4 \mathrm{~m}$ \\
Por punto & $16 \mathrm{~m}$ & $28 \mathrm{~m}$ \\
Por juego (s/6 puntos) & $96 \mathrm{~m}$ & $168 \mathrm{~m}$ \\
Por set (6 - 4) & $960 \mathrm{~m}$ & $1.680 \mathrm{~m}$ \\
Por partido (3 x 6 - 4) & $2.880 \mathrm{~m}$ & $5.040 \mathrm{~m}$ \\
\hline \multicolumn{2}{|c|}{ Los porcentajes por desplazamientos según superficies } \\
\hline \multicolumn{2}{|c|}{ Pista dura } & Tierra batida \\
\hline Frontal - diagonal & $25 \%$ & $14 \%$ \\
Lateral & $50 \%$ & $65 \%$ \\
Retroceder (atrás) & $6 \%$ & $6 \%$ \\
Sitio & $19 \%$ & $15 \%$ \\
\hline
\end{tabular}

Tabla 3. Tomada de: FUNDAMENTOS PRÁCTICOS DE LA PREPARACIÓN FÍSICA EN EL TENIS. Vila G, Carlos. Barcelona (2006. 12)

\subsection{Tipos de desplazamientos en el tenis.}

Siguiendo el tema importante que nos convoca "tipos de desplazamiento", en los siguientes puntos, vamos a encontrar un estudio de los diferentes tipos de desplazamientos que se realizan en una cancha de tenis cuando se está compitiendo. Estos tipos de desplazamiento son: hacia adelante, hacia atrás, laterales y hacia arriba; en los cuales, también están relacionada las hipotéticas distancias que se recorrerían en cada tipo de desplazamiento cuando se compite. Todo esto está determinado, por las dimensiones de la cancha, por el lugar en la cancha donde se juega mayormente y, en definitiva, por la mirada que debemos hacer desde la especificidad de los desplazamientos en el tenis y sus distancias recorridas para el estudio. 


\subsubsection{Desplazamientos hacia adelante.}

Estos son los desplazamientos que se hacen corriendo hacia el frente sin importar la dirección de la cancha, son los más utilizados en el tenis en número de veces y también suman la mayor distancia recorrida. Se utilizan para lograr mayor velocidad y llegar a impactar una pelota lejana del cuerpo que viene del otro lado de la cancha a gran velocidad. Estas acciones se pueden observar en los siguientes enunciados con sus respectivas imágenes graficadas de los desplazamientos típicos de frente.

- Desde un punto que queda por detrás de la línea divisoria de la línea de fondo a $1 \mathrm{~m}$ o $2 \mathrm{~m}$ cerca a la "T". Ver imagen 3.

- Desde la "T" hasta $1 \mathrm{~m}$ aproximado y a cualquiera de los dos lados de la intersección de la línea divisoria de los cuadros de servicio y la red divisoria de la cancha. Ver imagen 4.

- Desde la intersección de la línea de fondo con la línea de sencillos y a $1 \mathrm{~m}, 2 \mathrm{~m} \circ 3 \mathrm{~m}$ desde la línea imaginaria que sigue la línea de sencillos, hasta la "T". Ver imagen 5.

- Desde la intersección de la línea de fondo con la línea de dobles o, a $1 \mathrm{~m}, 2 \mathrm{~m}$ o $3 \mathrm{~m}$ desde la línea imaginaria que sigue la misma línea de dobles, hasta el punto imaginario a $1 \mathrm{~m}$ de distancia del punto que forma la línea divisoria de los cuadros de servicio con la red divisoria de la cancha. Ver imagen 6.

- Desde la intersección de la línea de fondo con la línea de dobles o, a $1 \mathrm{~m}, 2 \mathrm{~m}$ o $3 \mathrm{~m}$ desde la línea imaginaria que sigue la misma línea de dobles, hasta el punto imaginario a $1 \mathrm{~m}$ de distancia del punto de intersección que forma la línea de sencillos con la red del mismo lado de la cancha. ver imagen 7 .

- Desde un punto imaginario a $1 \mathrm{~m}, 2 \mathrm{~m} \circ 3 \mathrm{~m}$ por detrás de la línea divisoria de la línea de fondo, hacia un punto imaginario a $1 \mathrm{~m}, 2 \mathrm{~m} \circ 3 \mathrm{~m}$ de la continuación de la línea de dobles y $1 \mathrm{~m} \circ 2 \mathrm{~m}$ por fuera de esta línea imaginaria de dobles. Ver imagen 8. 
- Desde un punto imaginario a $1 \mathrm{~m}, 2 \mathrm{~m} \circ 3 \mathrm{~m}$ por detrás de la línea divisoria de la línea de fondo, hacia el punto que forma la intersección de la línea de servicio con cualquiera de la línea de sencillos. Ver imagen 9.

- Desde un punto imaginario a $1 \mathrm{~m}$ o $2 \mathrm{~m}$ de la intersección de la línea de servicio con cualquiera de la línea de sencillos, hasta un punto imaginario a $1 \mathrm{~m}$ o $2 \mathrm{~m}$ cerca a la intersección de la línea divisoria de los cuadros de servicio con la red. Ver imagen 10.

- Desde un punto a $1 \mathrm{~m}$ de la red, hasta $1 \mathrm{~m} \circ 4 \mathrm{~m}$ atrás de la línea de fondo. Ver imagen 11.

- Desde un punto a $3 \mathrm{~m}$ por detrás de la línea de fondo y al lado de la línea imaginaria de la línea de sencillos, hasta $1 \mathrm{~m}$ de la línea de fondo al lado de la línea de sencillos. Ver imagen 12.

- Desde un punto siguiendo la línea imaginaria de sencillos hacia atrás $1 \mathrm{~m}, 2 \mathrm{~m} \circ 3 \mathrm{~m}$, hasta la intersección de la otra línea de sencillos con la línea de los cuadros de servicio. Ver imagen 13.

- Desde un punto siguiendo la línea imaginaria de sencillos o dobles a $1 \mathrm{~m}$ o $2 \mathrm{~m}$; hasta la otra línea imaginaria de la línea de sencillos o dobles a $1 \mathrm{~m} \circ 2 \mathrm{~m}$ hacia atrás. Ver imagen 14.

\subsubsection{Desplazamientos laterales.}

Los desplazamientos laterales son muy usados en el tenis, pues lo jugadores suelen quedarse en la mayoría de los impactos, detrás de la línea de fondo y por ende deben desplazarse hacia los lados extremos de la cancha o hacia al centro de la misma después de un golpe de derecha o revés. Estos desplazamientos laterales son rápidos, explosivos y de ajuste para nuevamente acomodarse y prepararse para el siguiente tiro.

- Dese la intersección de la línea de fondo con la línea de sencillos o, a $1 \mathrm{~m}, 2 \mathrm{~m} \circ 3 \mathrm{~m}$ por la misma línea imaginaria de sencillos; hasta la intersección con la línea imaginaria de la línea media de fondo o, a 1m, $2 m$ o $3 m$ cerca de ella. Ver imagen 15. 
- Desde un punto de la línea imaginaria que sale de la línea media de fondo hacia atrás a $1 \mathrm{~m}, 2 \mathrm{~m} \circ 3 \mathrm{~m}$, hasta un punto a,1m, $2 \mathrm{~m} \circ 3 \mathrm{~m}$ cerca de la línea imaginaria de la línea de sencillos y paralelos a la línea de fondo. Ver imagen 16.

- Desde un punto sobre la línea media de la línea de fondo o a $1 \mathrm{~m}$ de ella hacia el fondo, hasta un punto medial y cercano a $1 \mathrm{~m} \circ 2 \mathrm{~m}$ de la línea de servicio. Ver imagen 17.

- Desde un punto imaginario cerca de $1 \mathrm{~m}$ a la "T" o sobre ella, hasta $1 \mathrm{~m}$ o $2 \mathrm{~m}$ cerca de la intersección entre la línea de sencillos y la línea de servicio. Ver imagen 18.

- Desde un punto a cualquiera de los dos lados de la línea divisoria de los cuadros de servicio a $1 \mathrm{~m}$ o $2 \mathrm{~m}$ cerca a la red, hasta $1 \mathrm{~m}$ o $2 \mathrm{~m}$ paralelo a la red y cerca de $1 \mathrm{~m}$ o $2 \mathrm{~m}$ de la línea de sencillos. Ver imagen 19.

- Detrás y a un lado de la continuación de la línea de sencillos, hacia $1 \mathrm{~m}$, $2 m$ o $3 m$ hacia el medio de la cancha o hacia el lado de la continuación de la línea de dobles. Ver imagen 20.

\subsubsection{Desplazamientos hacia atrás.}

Los desplazamientos hacia atrás en el tenis, se realizan como método de reacomodación a un tiro proveniente del otro lado ya sea; un servicio, un golpe profundo cuando la pelota caerá cerca de nuestro cuerpo o porque vemos que la pelota lleva una trayectoria en forma de parábola lenta y el jugador se acomoda haciendo unos pasos hacia tras para impactar casi siempre con el mejor golpe que es, su derecha.

- Desde un punto a $1 \mathrm{~m}$ al frente o al lado de la línea divisoria de la línea de fondo, hasta $1 \mathrm{~m} \circ 2 \mathrm{~m}$ por fuera de la cancha hacia atrás de la línea divisoria de la línea de fondo. Ver imagen 21.

- Sobre un punto de la línea media de fondo o a $1 \mathrm{~m}$ o $2 \mathrm{~m}$ en continuación con la línea imaginaria de la misma línea, hasta, $1 \mathrm{~m}$ o $2 \mathrm{~m}$, cerca de la línea imaginaria de la línea de sencillos. Ver imagen 22. 
- En un punto sobre la "T", hacia atrás a $1 \mathrm{~m}, 2 \mathrm{~m} \circ 3 \mathrm{~m}$. Ver imagen 23.

- En un punto a $1 \mathrm{~m}$ dentro de la cancha y cerca de la línea media de fondo, hacia atrás a $1 \mathrm{~m}$ detrás de la línea de fondo. Ver imagen 24.

\subsubsection{Desplazamientos hacia arriba o saltos.}

Estos desplazamientos que son propios de la gran mayoría de los deportes, sea individuales o de conjunto, se utiliza para alcanzar una altura que permita ejecutar una acción compleja, iniciante, para mantener o culminante de un juego; es decir, estos movimientos hacia arriba en el tenis se hace para impactar la pelota que vine en una trayectoria ascendente en forma de parábola y el jugador la tendrá que impactar lo más alto que le sea posible e imprimir toda su potencia para pasar del otro lado la pelota con el fin de que no sea devuelta.

Los tipos de golpes que se ejecutan realizando desplazamientos hacia arriba, son: el servicio (saque) y el smash. Aunque este desplazamiento se utiliza primordialmente para estos tipos de golpe; otros golpes como: el drive, el revés y la volea con swing, se ejecutan con un pequeño desplazamiento hacia arriba producto de la sinergia del movimiento proveniente del impulso que dan los pies en la cadena cinética de movimiento al cuerpo para finalizar con el impacto con la pelota.

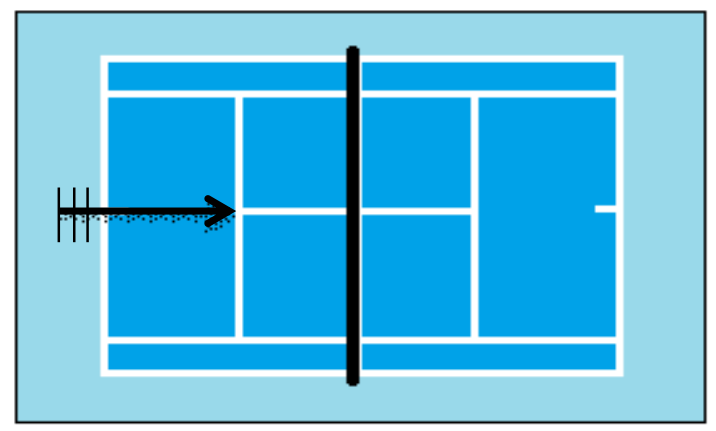

Imagen 3. Distancia aproximada de $6,485 \mathrm{~m}$ a $7,485 \mathrm{~m}$ de desplazamiento frontal.

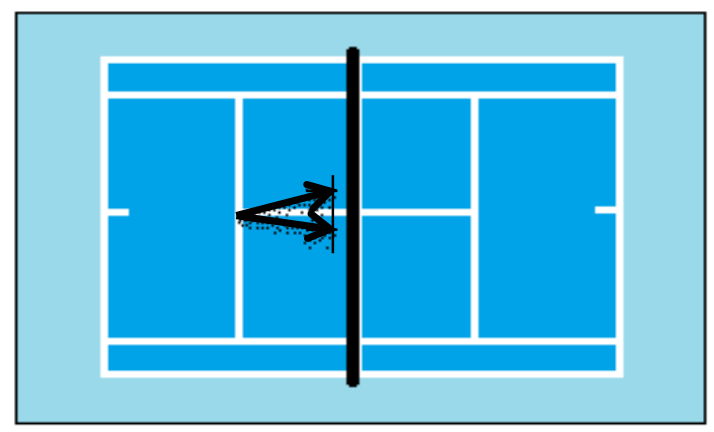

Imagen 4. Distancia aproximada de $5,40 \mathrm{~m}$ de desplazamiento frontal. 


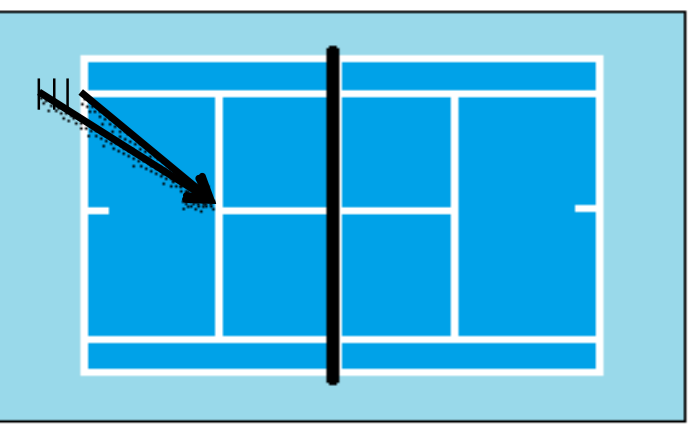

Imagen 5. Distancia aproximada de $6,85 \mathrm{~m}$ a $9,85 \mathrm{~m}$ de desplazamiento frontal.

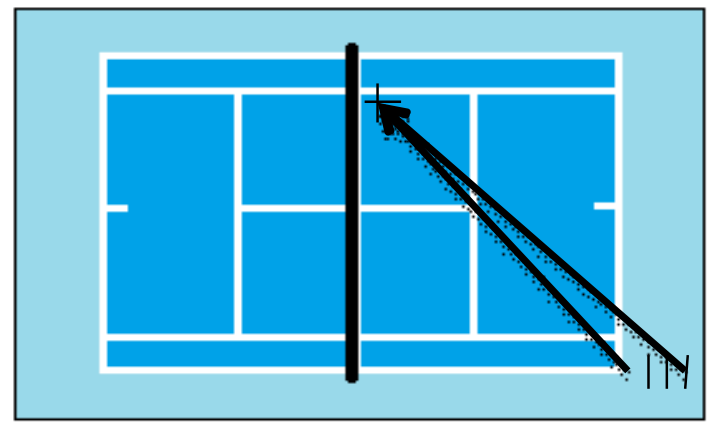

Imagen 7. Distancia aproximada de $14,48 \mathrm{~m}$ a $17,48 \mathrm{~m}$ de desplazamiento frontal.

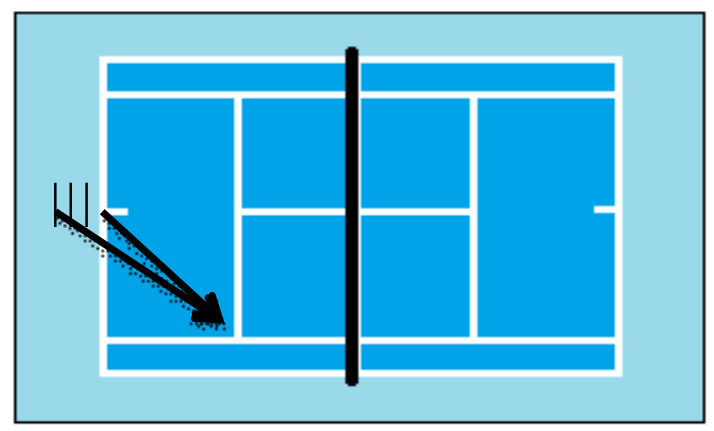

Imagen 9. Distancia aproximada de $6,84 \mathrm{~m}$ a $9,84 \mathrm{~m}$ de desplazamiento frontal.

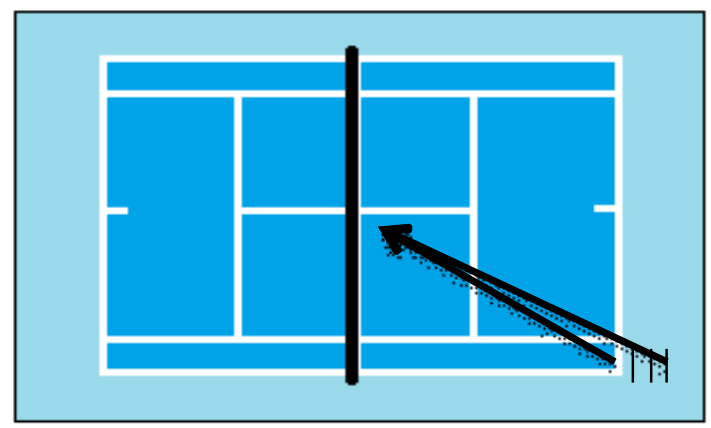

Imagen 6. Distancia aproximada de $11,54 \mathrm{~m}$ a $14,54 \mathrm{mts}$ de

Desplazamiento frontal.

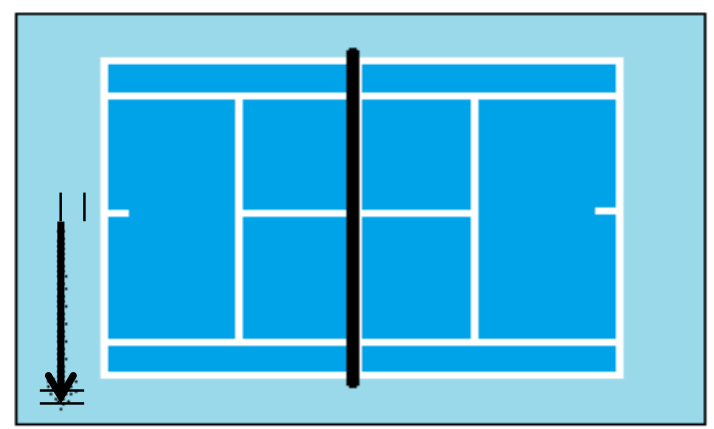

Imagen 8. Distancia aproximada de $5,48 \mathrm{~m}$ a $7,48 \mathrm{~m}$ de desplazamiento frontal.

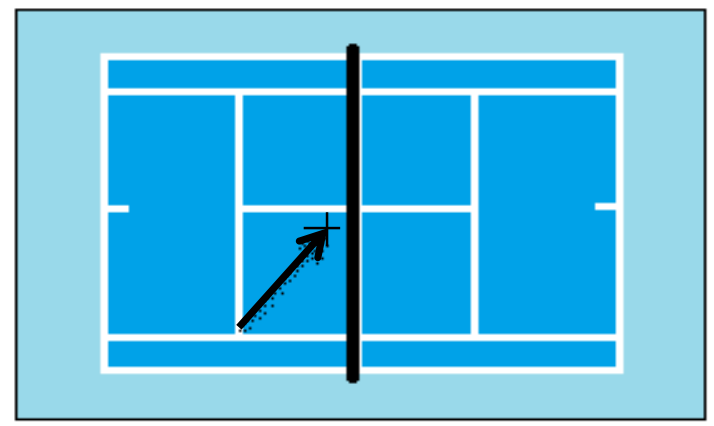

Imagen 10. Distancia aproximada de $6,23 \mathrm{~m}$ de desplazamiento frontal. 


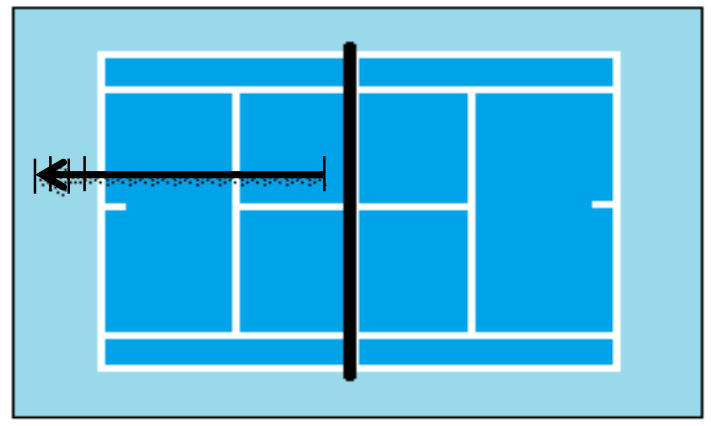

Imagen 11, distancia aproximada de $10,85 \mathrm{~m}$ a $14,85 \mathrm{~m}$ de desplazamiento frontal.

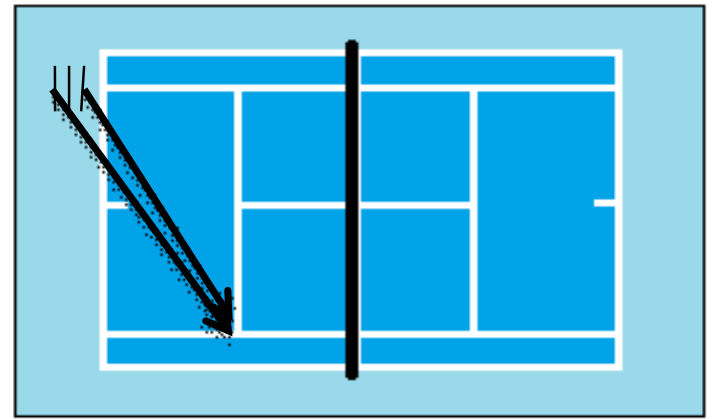

Imagen 13. Distancia aproximada de $9,81 \mathrm{~m}$ a $11,81 \mathrm{~m}$ desplazamiento frontal.

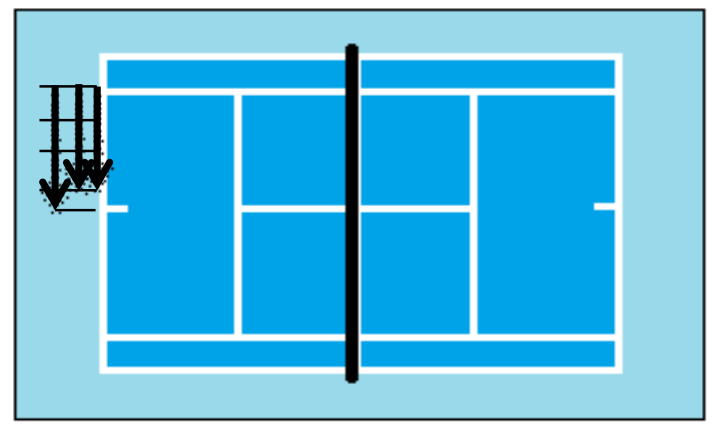

Imagen 15. Distancia aproximada de $1 \mathrm{~m}$ a $4 \mathrm{~m}$ de desplazamiento lateral.

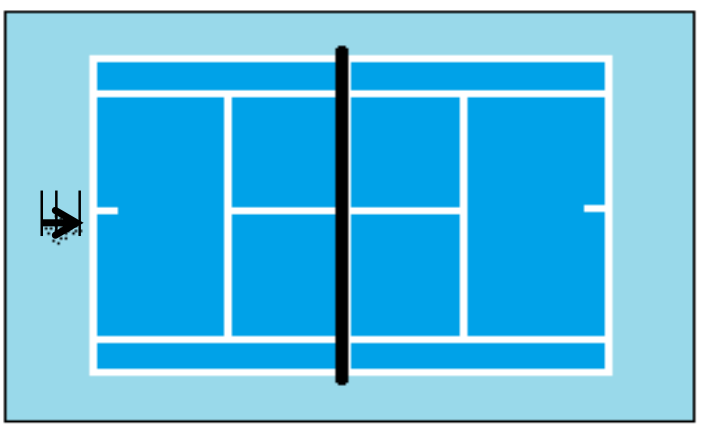

Imagen 12. Distancia aproximada de $1 \mathrm{~m}$ a $2 \mathrm{~m}$ de desplazamiento frontal.

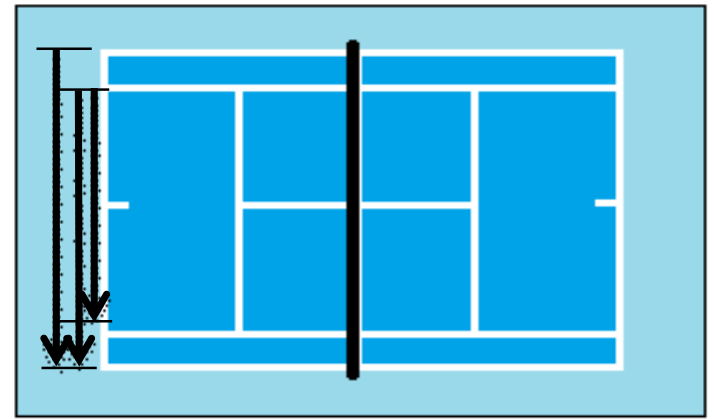

Imagen 14. Distancia aproximada de $8,23 \mathrm{~m}$ a $10,97 \mathrm{~m}$ desplazamiento frontal.

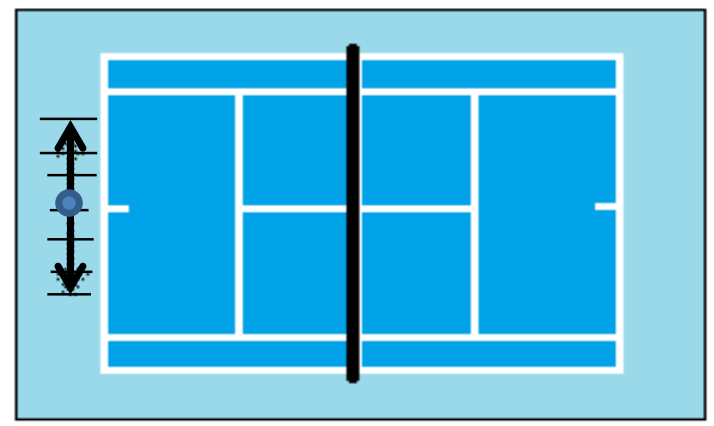

Imagen 16. Distancia aproximada de $1 \mathrm{~m}$ a $3 \mathrm{~m}$ de desplazamiento lateral. 


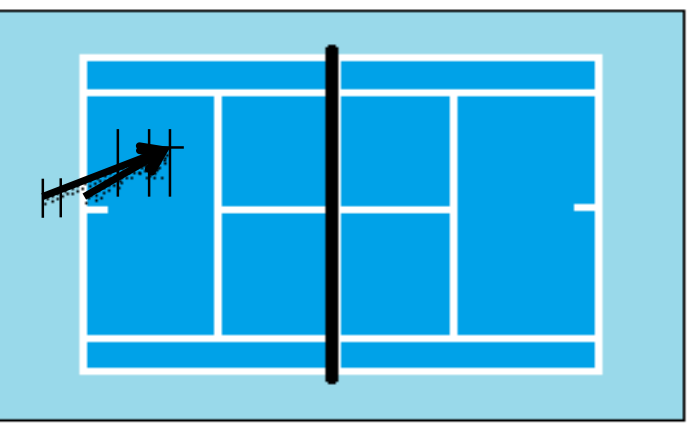

Imagen 17. Distancia aproximada de $3 \mathrm{~m}$ a $4 \mathrm{~m}$ de desplazamiento lateral.

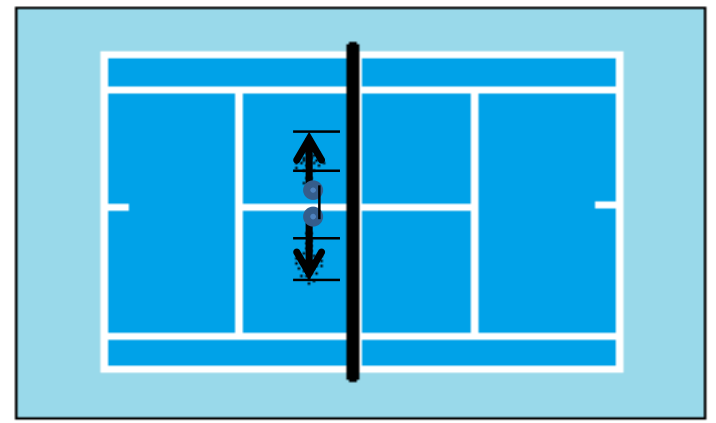

Imagen 19. Distancia aproximada de $1 \mathrm{~m}$ a $3 \mathrm{~m}$ de desplazamiento lateral.

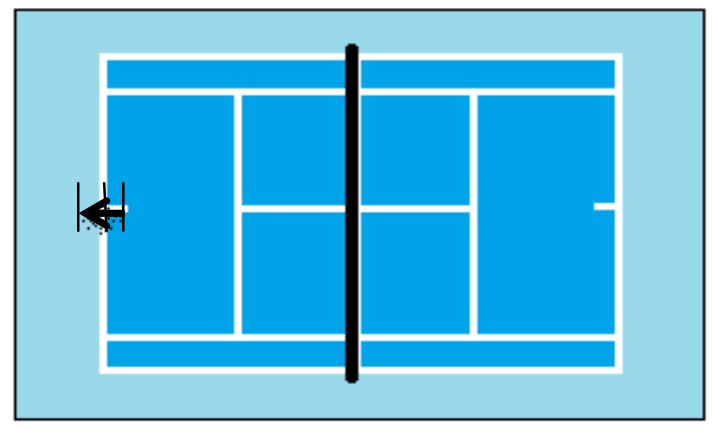

Imagen 21. Distancia aproximada de $1 \mathrm{~m}$ a $2 \mathrm{~m}$ de desplazamiento hacia atrás.

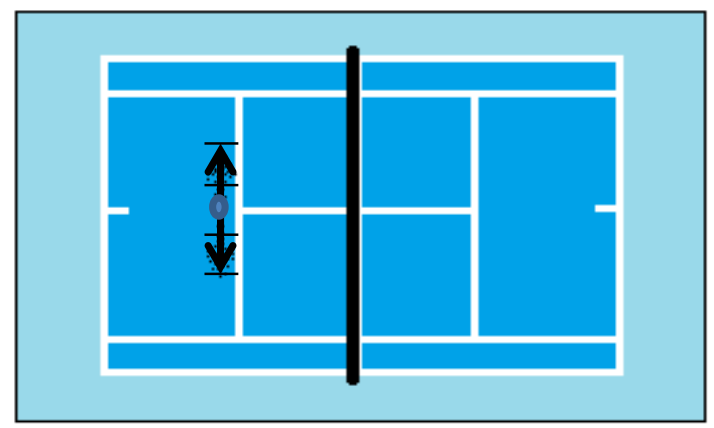

Imagen 18. Distancia aproximada de $1 \mathrm{~m}$ a $2 \mathrm{~m}$ de desplazamiento lateral.

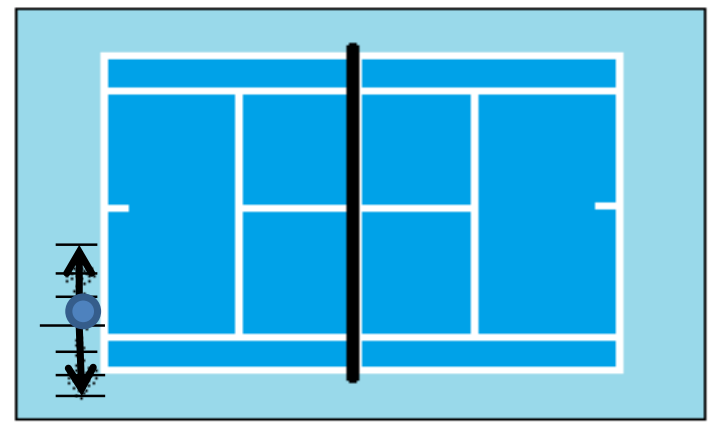

Imagen 20. Distancia aproximada de $1 \mathrm{~m}$ a $3 \mathrm{~m}$ de desplazamiento lateral.

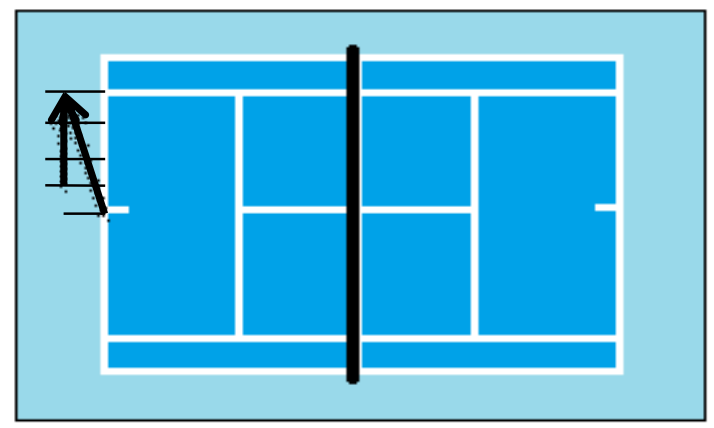

Imagen 22. Distancia aproximada de $1 \mathrm{~m}$ a $4 \mathrm{~m}$ de desplazamiento hacia atrás. 


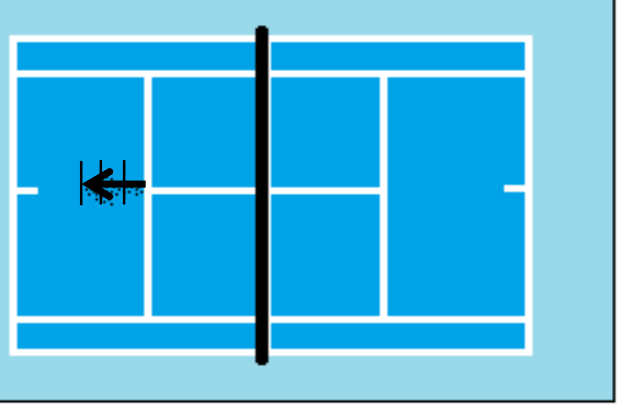

Imagen 23. Distancia aproximada de $1 \mathrm{~m}$ a $3 \mathrm{~m}$ de desplazamiento hacia atrás.

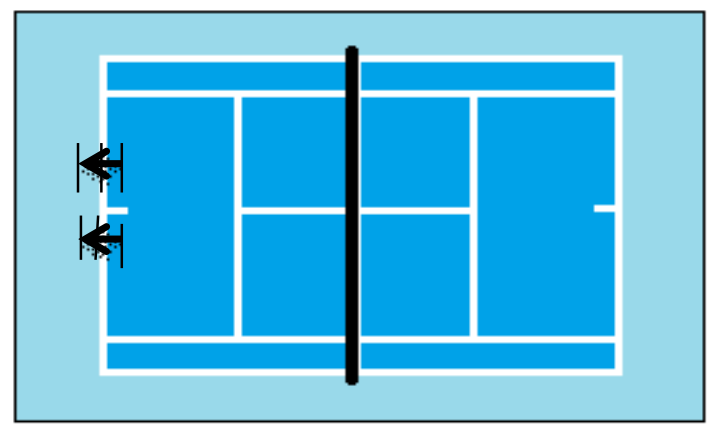

Imagen 24. Distancia aproximada de $1 \mathrm{~m}$ a $2 \mathrm{~m}$ de desplazamiento hacia atrás.

\subsection{Relación entre el bote de la pelota y los diferentes tipos de cancha.}

Para referirnos a la relación que existe entre el bote de la pelota de tenis y los diferentes tipos de pistas o canchas de tenis, y que a partir de allí podamos sacar algunas conclusiones para el posterior estudio y por último hacer las propuestas correspondientes a la tesis sobre el "Acondicionamiento Físico Precompetitivo para el Tenis"; debemos entonces, interpretar las siguientes consideraciones de los diferentes tipos de pista y lo que sucede en cada una de ellas cuando se juega.

Según la ITF (2014. 21), el método de prueba utilizado para determinar la velocidad de la superficie de una pista es el método ITF CS 01/02, como se describe en la publicación de la ITF titulada "ITF guide to test methodsfor tennis courtssurfaces" (Guía de la ITF de métodos de prueba sobre las superficies de las pistas de tenis). Estos métodos de prueba determinan si una cancha es rápida o no; pero lo más importante para rescatar, es que, desde esta perspectiva, se deba tener en cuenta: la velocidad con la que vendrá dado el juego y la velocidad con la que la pelota bota después de tocar cierto tipo de pista.

Con lo anterior, hay que tener en cuenta también, la velocidad con la que haya que preparar el golpe, acomodarse, responder y prepararse para el siguiente 
golpe en los diferentes tipos de pista. Todo esto, entonces, se debería proponer y tener presente que las disposiciones de cada tipo de cancha, requiere sin lugar a dudas, un tipo de preparación específica para el deportista antes de entrar a la competición.

Tenemos así, que: las superficies que tengan una clasificación de la ITF entre 0 y 29 serán clasificadas dentro de la Categoría 1 (velocidad baja). Ejemplos de tipos de superficies que se ajustan a esta clasificación incluirán la mayoría de las pistas de tierra batida y otros tipos de superficies con minerales sueltos ITF (2013. 21). En este tipo de pista, el bote de la pelota será más lento y toma más altura; los jugadores pueden deslizarse para llegar a todos los tiros de pelota. El tipo de juego suele ser más trabajado detrás de la línea de fondo, con una mayor cantidad de pases sobre la red y puntos más largos, lo que requiere mayor desgaste físico. El torneo más famoso del mundo sobre tierra batida es Roland Garros, en París destacando también el Madrid Open.

En estos tipos de superficies, los botes de la pelota suelen ser más altos por dos razones, 1, el jugador tiene más tiempo para preparar el golpe e imprimirle más aceleración y efecto a la pelota; 2 , la pista absorbe las inercias lineales de la pelota y también genera más contacto con ella. Estas dos características, hacen que la pelota se eleve más al caer del otro lado después de un golpe, y por ende, la persona que responde tendrá que hacer el gesto mayormente a la altura de la parte alta del tronco o los hombros. Ver imagen 25.

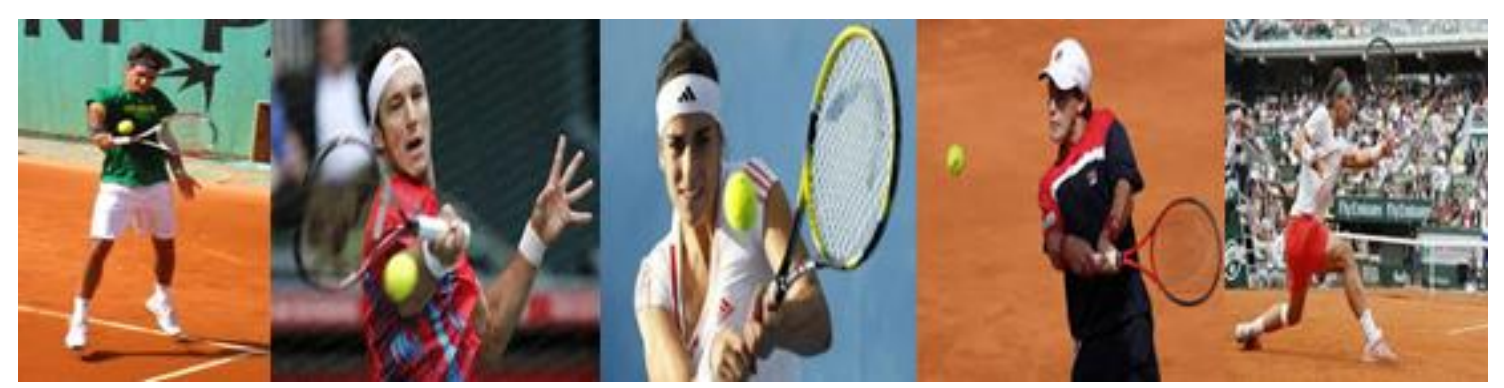

Imagen 25. Imágenes de diferentes jugadores en el momento de golpeo en canchas de polvo de ladrillo. 
Por otro lado, tenemos: las superficies que tengan una clasificación de la ITF entre 30 y 34 que serán clasificadas dentro de la Categoría 2 (velocidad mediabaja), mientras que las comprendidas entre 35 y 39 estarán en la Categoría 3 (velocidad media). Ejemplos de tipos de superficies que se ajustan a esta clasificación incluirán a la mayoría de superficies de capa acrílica más algunas superficies de mosqueta ITF (2013. 21). Las pistas de tenis duras o de cemento están proliferando de manera considerable en el tenis moderno. En cuanto a rapidez suponen el punto medio entre la lentitud de las de arcilla y la velocidad de las de hierba, por lo que son típicos los puntos cortos que favorecen a los jugadores que tienen un buen saque. El Abierto de Estados Unidos y el de Australia son los principales torneos.

Los tipos de superficie de velocidad media como estos, son los más utilizados últimamente en los grandes torneos y en la mayoría de torneos master 250, master 500 y master 1000; pues si vemos, hay dos Grand Slam que se juegan en estos tipos de pistas, uno a comienzo de año que es en Australia y el otro finalizando el segundo cuatrimestre del año en Estados unidos. Pero lo más importante es el bote de la pelota y la velocidad de la misma. El bote de la pelota es uniforme por el aspecto casi liso de la pista; la altura promedio de impacto de una pelota en estas pistas suele ser a la altura de la cadera y parte baja del tronco. Ver imagen 26.

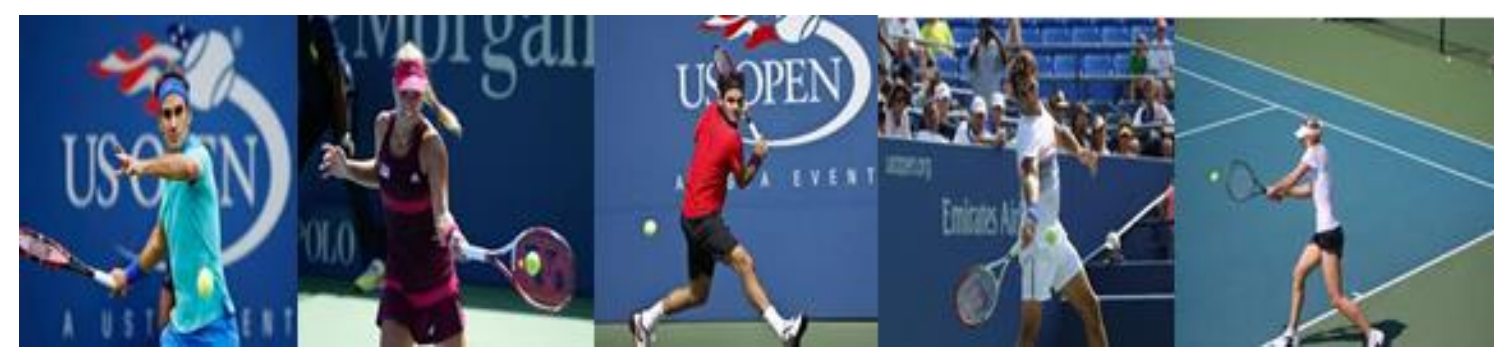

Imagen 26. Imágenes de jugadores en el momento de golpeo en canchas de cemento.

Por último, nos remitiremos quizás la pista más difícil para jugar al tenis. Según la ITF $(2014,21)$, las superficies que tengan una clasificación entre 40 y 44 
serán clasificadas dentro de la Categoría 4 (velocidad media-alta), mientras que las de 45 o más estarán en la Categoría 5 (velocidad alta). Ejemplos de tipos de superficies que se ajustan a esta clasificación incluirán la mayoría de las pistas de hierba natural, hierba artificial y algunas superficies de mosqueta. Tenemos que Wimbledon en Inglaterra es el torneo representante de este tipo de terreno.

Estas pistas son pistas muy rápidas en relación con el juego y los botes de la pelota suelen ser irregulares, bajos y muy rápidos; lo cual es la característica principal de este terreno, pues no significa que solo la pelota bota bastante bajo y de forma rápida sino que cambia constantemente de altura y dirección, provocando muchos errores de ejecución y exige mucho más físicamente las extremidades inferiores, y encontramos también que los impactos de la pelota suelen estar a la altura de la rodilla y la cadera. Ver imagen 27.

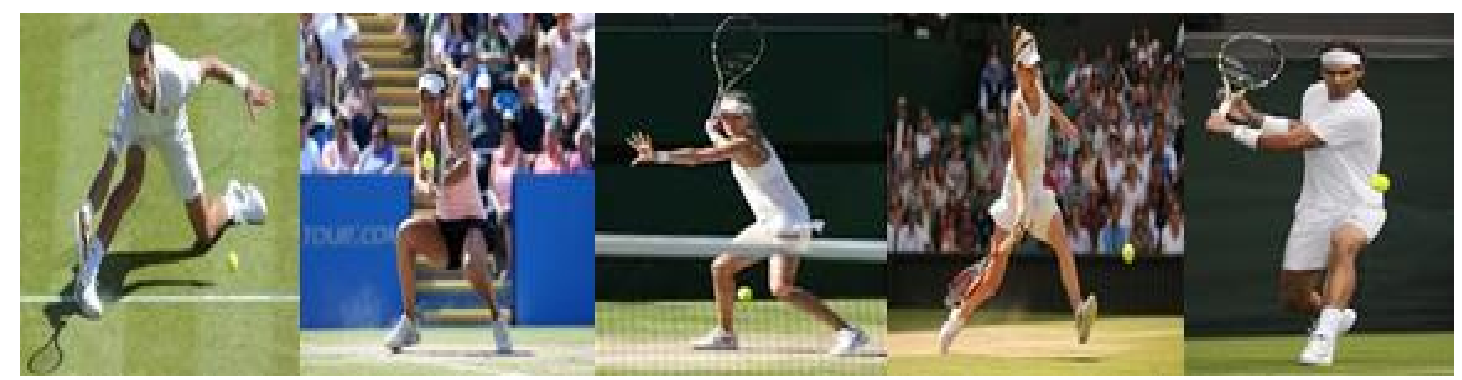

Imagen 27. Imágenes de jugadores y jugadoras en el momento de golpeo en canchas de hierba o césped.

\subsection{Análisis de las competiciones en tenis.}

El análisis de las competiciones en el tenis, recientemente se han utilizado de manera exponencial, dado al avance deportivo en cuanto a la consecución de más y mejores marcas; tomando partido de todos los factores que envuelven al deporte. Estos factores hacen parte del deportista, lo afectivo o emocional, lo social y lo biológico; a este último, aun mucho más se le ha intervenido para el avance de las capacidades más palpables y observables en el ámbito deportivo. Es así que los entrenadores planifican de acuerdo a los tipos de 
movimientos (técnicas), frecuencia de esos movimientos (número de repeticiones) y, de acuerdo a esto, el volumen a trabajar.

La necesidad aquí en nuestra tesis, es investigar los desplazamientos y los tiempos de trabajo pausa. Estas características, hace que nuestra investigación tome un sentido más real y directo con lo que tiene que ver en la propuesta de "Acondicionamiento Físico Precompetitivo para el Tenis" y, por tal motivo, tenemos que observar, anotar y después diagramar tablas de los contenidos observados en cada uno de los desplazamientos y cada uno de los momentos de trabajo y pausa del tenista o la tenista en la competición. Aspecto para nada fácil de hacer, pues la observación debe ser minuciosa y detallada al contabilizar los metros recorridos en cada desplazamiento, el tipo de desplazamientos que el jugador o jugadora utilizó y luego sacar los promedios y porcentajes; pues no hay hasta ahora un software que contabilice los tipos de desplazamientos.

Con lo anterior, hemos encontrado en la bibliografía del tenis, unos datos que nos acercan a lo que ha sido una estadística específica de lo que pasa en un partido de tenis. Es muy importante notar al respecto, que, las investigaciones siempre vienen bien para el desarrollo de la actividad que se está practicando, como también, aporta nuevas perspectivas para seguir adelante con nuevas investigaciones en el mismo campo en busca de la más mínima variabilidad para seguir evolucionando. Ahora bien, según las estadísticas publicadas en 1988 por la ATP (Asociación de Tenistas Profesionales), basadas en un estudio realizado entre los 150 primeros clasificados del ranking de la ATP de partidos jugados sobre polvo de ladrillo, tenemos los siguientes datos estadísticos:

Duración media de un punto.

Duración media de un punto (en pista rápida).

Tiempo real de juego.

Promedio de puntos por juego.

Promedio de puntos por set.

Promedio de puntos de un partido de 5 sets.

Distancia media recorrida por golpe (con saque).

Distancia media recorrida por golpe (sin saque).
6-10 segundos.

4'3 segundos.

$22 \%$ del total.

$6 ' 2$.

62.

310.

3 metros.

3'8 metros. 
Distancia media recorrida por punto.

Distancia media recorrida en un set.

Distancia media recorrida en un partido de 5 sets.

El $40 \%$ de distancia se hace caminando.

El $47 \%$ de desplazamientos son hacia delante.

El $48 \%$ son laterales.

El $5 \%$ hacia atrás.

Consumo medio de energía en un parido de 5 sets.

Consumo medio de oxígeno.

Promedio del ritmo cardíaco durante el partido (hombres). 143ppm.

Promedio de ritmo cardíaco durante el partido (mujeres). $153 \mathrm{ppm}$.
8-12 metros.

850 metros.

4.250 metros.

Aunque este análisis haya sido del siglo pasado, y estamos hablando de 1998, es un punto de partida y se acerca mucho a lo que hoy en día se sigue presentando en las competencias tenísticas, dado que solo han ocurrido algunos cambios minuciosos en los materiales como raquetas, pelotas y canchas, que han hecho variar la velocidad del juego. Por tal motivo, se hace indispensable, estudios nuevos como el que realizamos en esta tesis para determinar con más exactitud, lo nuevos tiempos de trabajo-pausa y los tipos de desplazamientos utilizados en el tenis; para así poder desarrollar nuevas conclusiones y diagramar a partir de esto algunas propuestas más específicas que apunten a la mejora del rendimiento deportivo.

\subsubsection{Análisis de competiciones en diferentes tipos de pistas.}

Para el análisis de estos partidos en diferentes tipos pistas o canchas $y$, teniendo en cuenta que solo se realizará el estudio de los primeros sets de algunos partidos masculinos y femeninos en los "Grand Slam, Master mil y ATP-250", vamos a tener presente las siguientes variables: tiempos de trabajo, tiempos de pausa y tipos de desplazamientos, (ver tabla 4). Después, teniendo como referencia este análisis y con algunas otras perspectivas que contemplan las variables propuestas, podremos sacar algunas conclusiones al respecto para tener en cuenta en nuestra propuesta de -Acondicionamiento Físico pre Competitivo-. 


\begin{tabular}{|lc|}
\hline TIPOS DE DESPLAZAMIENTOS & Guía \\
\hline \hline Desplazamiento hacia adelante. & $\mathrm{F}$ \\
Desplazamiento hacia atrás de ejecución. & $\mathrm{A}$ \\
Desplazamiento hacia atrás de recuperación. & $\mathrm{AA}$ \\
Desplazamiento lateral de ejecución. & $\mathrm{L}$ \\
Desplazamiento lateral de recuperación. & $\mathrm{Lr}$ \\
Desplazamiento hacia arriba o salto. & $\mathrm{S}$ \\
\hline
\end{tabular}

Tabla 4.

Estos análisis de los "primeros sets" de los partidos de tenis, tienen en cuenta también la clasificación de los tipos de pista o cancha que determina la velocidad de la superficie según el método ITF CS 01/02, que son las siguientes: "velocidad baja $(0-29)$, velocidad media $(35-39)$ y velocidad alta $(45$ - <)". Entonces, para tal estudio, se tomará como referencia, dos partidos por cada superficie, uno de mujeres y uno de hombres respectivamente; que en total será un estudio de 6 partidos diferentes en tres tipos diferentes de cancha $y$, en torneos de élite 5 Grand Salam y 1 jugado en un ATP-250.

En las siguientes páginas, vamos a presentar los estudios de cada uno de los tipos de desplazamientos realizados y los tiempos de trabajo-pausa en el primer set de cada partido; para esto, desarrollaremos unos tipos de tablas que englobarán lo que pasa en cada "Game" del set y hacer más fácil su estudio y compresión.

1. Análisis punto a punto del primer set del partido entre DominiKa Cibulkova (Slo) y Li Na (Chi), en la final del Grand Salam Australia Open 2014; en el cual, $\mathrm{Li} \mathrm{Na}$ es la ganadora 7-5 / 6-0. Tipo de superficie, cancha (dura), clasificación ITF categoría 3 (velocidad media).ver tablas 6 a 23.

- Análisis de los tiempos y tipos de golpes ejecutados dentro de la cancha antes de comenzar el partido (peloteo previo al partido):

Antes de comenzar el partido, las jugadoras entran a la cancha, realizan los actos protocolarios y luego comienzan a pelotear detrás de la línea de fondo de la cancha a intensidad media haciendo pasar la pelota por la mitad de la 
cancha para entrar en ritmo de juego durante 1,42'. Luego D. Cibulkova se acerca a dos pasos de la red para volear hasta el minuto 2' y enseguida, situarse sobre la "T" para hacer Smash hasta el minuto 2,10'. En este momento se intercambian los roles y $\mathrm{N}$. Li pasa a volear también cerca a la red, mientras Cibulkova pelotea desde el fondo de la cancha hasta el minuto 2,46' en el que Li comienza hacer smash hasta el minuto 3'; momento en el que las dos jugadoras pasan a situarse en el fondo de la cancha para realizar servicios cruzados a cada lado hasta el minuto 5,25'. Por último, van a sus sillas, descansan, beben y alistan la raqueta para empezar el partido hasta el minuto 6,18 ' después de que comenzaron a pelotear. Ver tabla 5.

\begin{tabular}{|c|c|c|c|c|}
\hline \multicolumn{6}{|c|}{$\begin{array}{r}\text { ANÁLISIS DEL USO DE LOS TIPOS DE GOLPES EN EL MOMENTO PREVIO AL } \\
\text { COMIENZO DEL PARTIDO(PELOTEO PREVIO AL PARTIDO) }\end{array}$} \\
\hline Tipo de golpe & D Cibulkova & L Na & Tiempo promedio & $\%$ \\
\hline $\begin{array}{c}\text { (derecha y revés) Peloteo } \\
\text { de fondo de cancha }\end{array}$ & $\begin{array}{c}102 "+36 "+14 " \\
=152 "\end{array}$ & $130 "$ & $141 "$ & $43,38 \%$ \\
\hline Volea cerca a la red & $18 "$ & $36 "$ & $27 "$ & $8,30 \%$ \\
\hline Smash sobre la "T" & $10 "$ & $14 "$ & $12 "$ & $3,69 \%$ \\
\hline Servicio o saque & $145 "$ & $145 "$ & $145 "$ & $44,61 \%$ \\
\hline
\end{tabular}

Tabla 5.

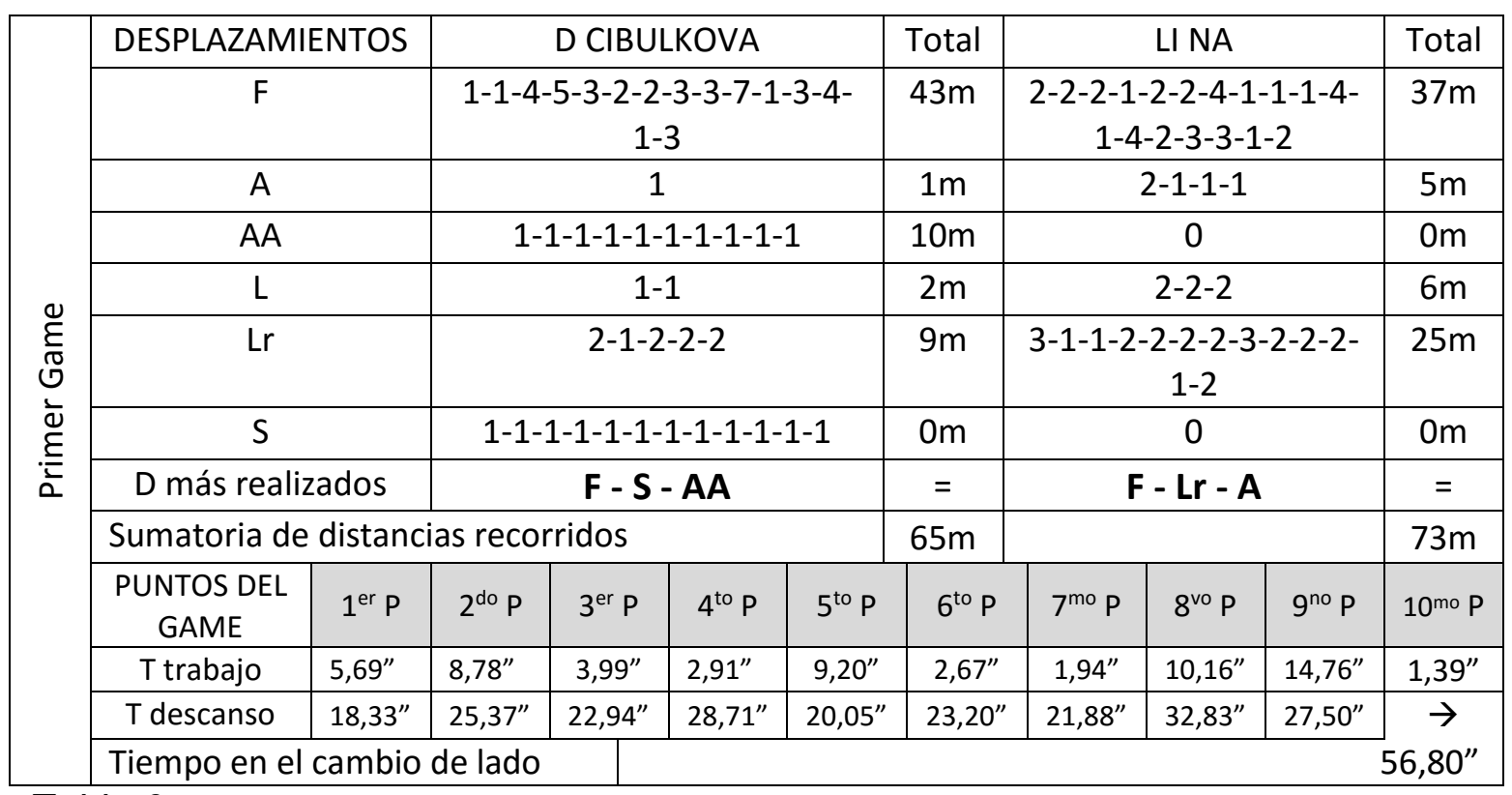

Tabla 6. 


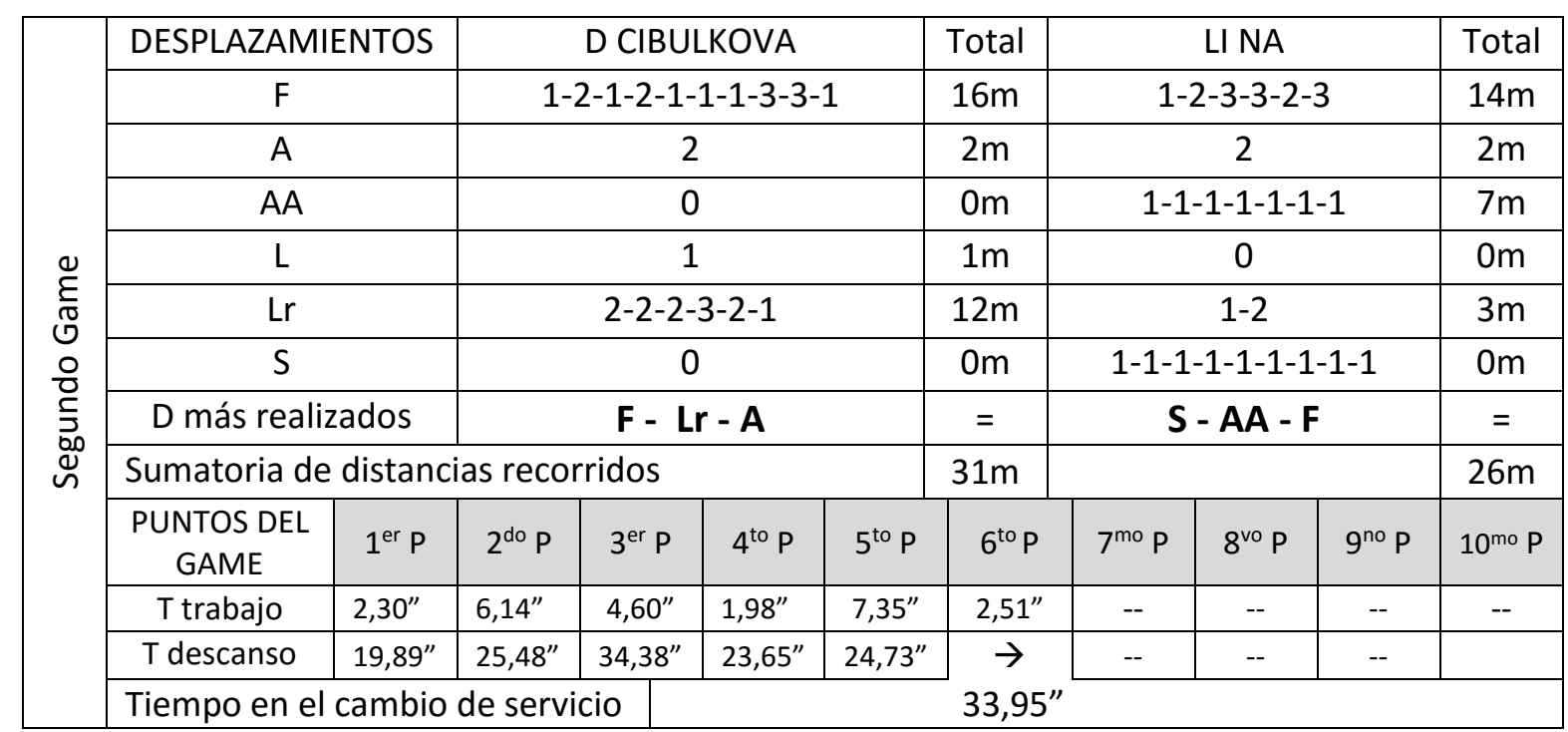

Tabla 7.

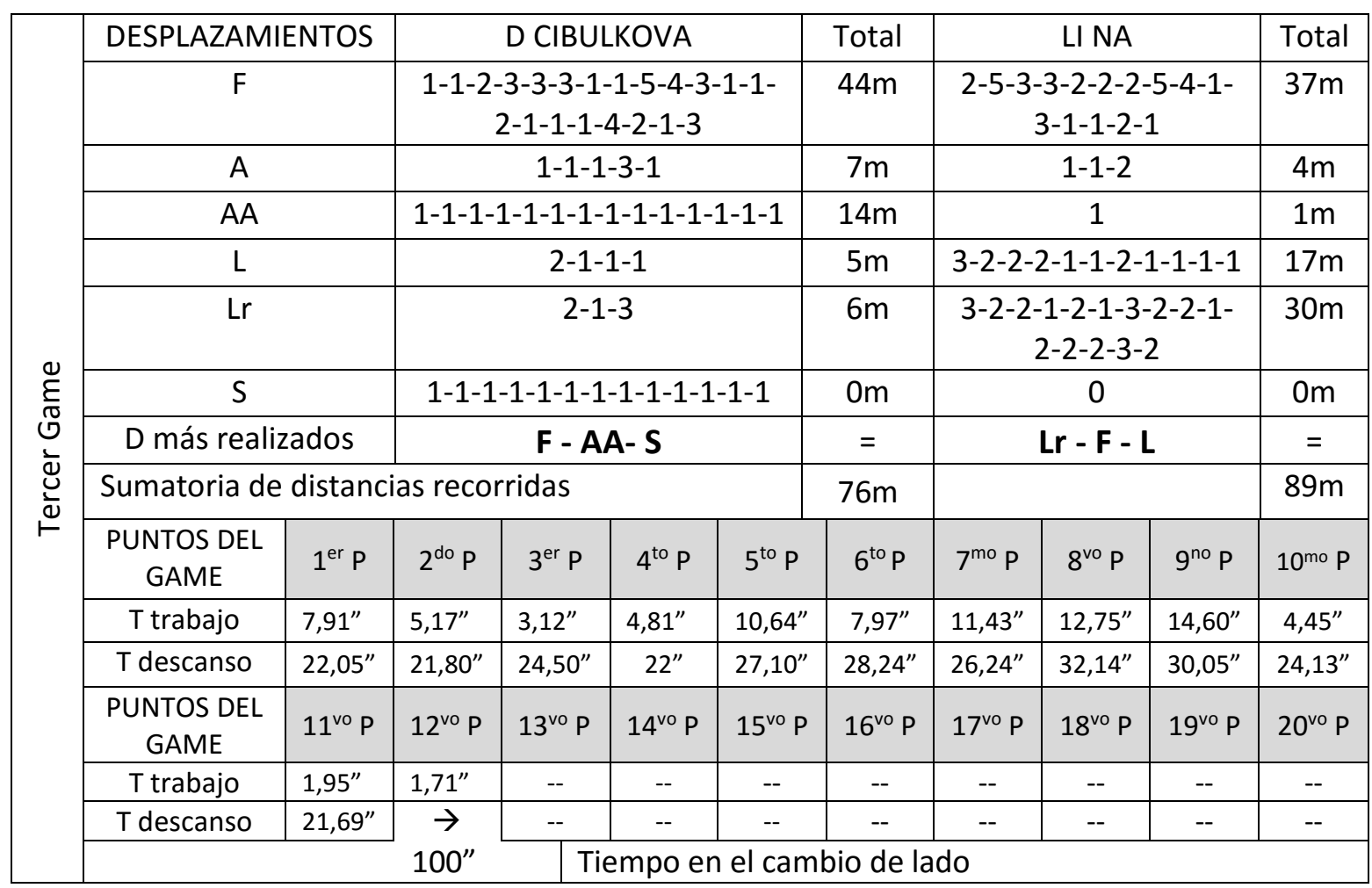

Tabla 8. 


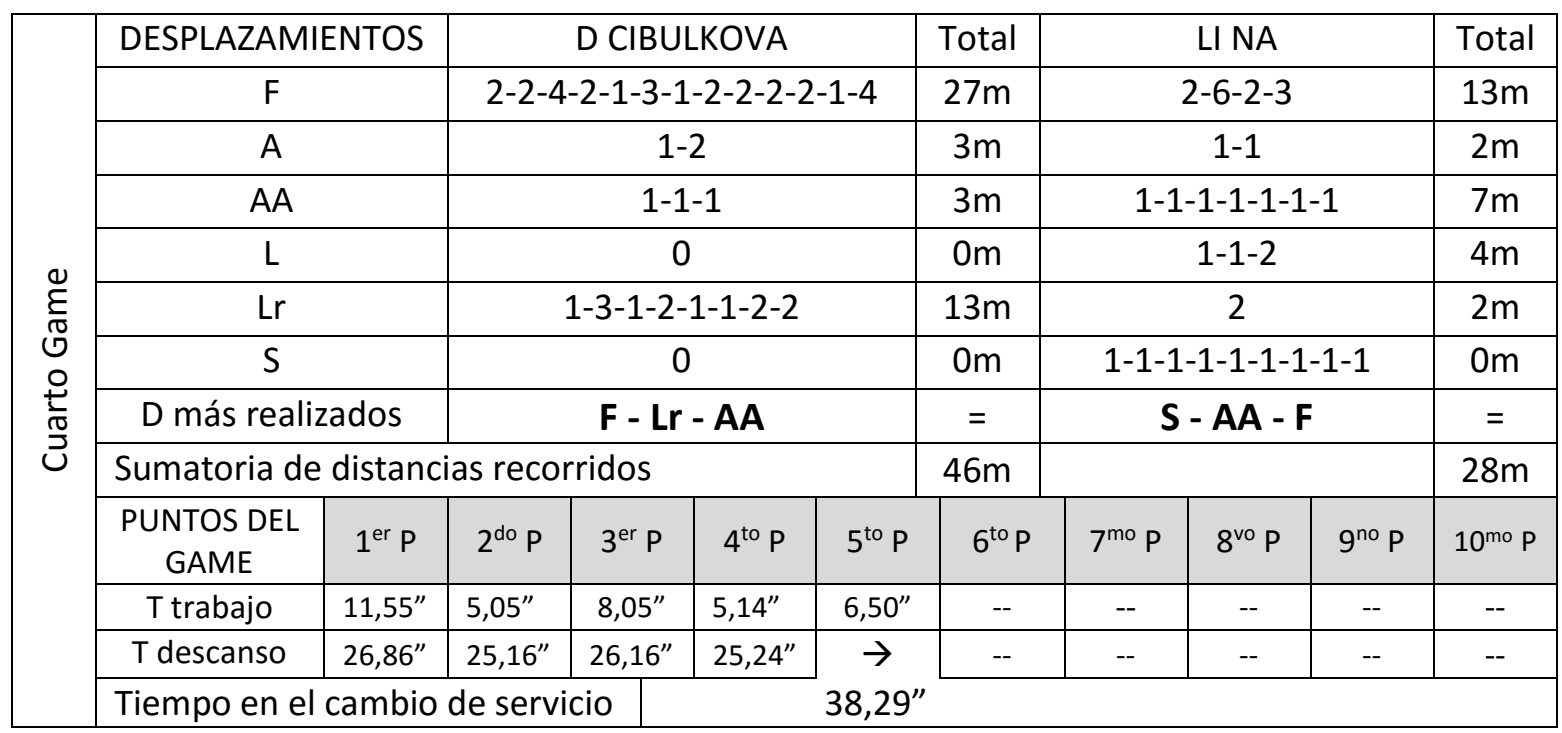

Tabla 9.

\begin{tabular}{|c|c|c|c|c|c|c|c|c|c|c|c|}
\hline \multirow{13}{*}{ 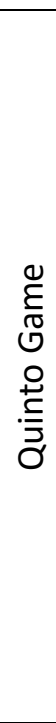 } & \multicolumn{2}{|c|}{ DESPLAZAMIENTOS } & \multicolumn{4}{|c|}{ D CIBULKOVA } & Total & \multicolumn{3}{|c|}{ LI NA } & Total \\
\hline & \multicolumn{2}{|l|}{$\mathrm{F}$} & \multicolumn{4}{|c|}{$3-3-2-1-3-2-4-2$} & $20 \mathrm{~m}$ & \multicolumn{3}{|c|}{ 1-1-2-1-1-1-2-3-1-1 } & $14 \mathrm{~m}$ \\
\hline & \multicolumn{2}{|l|}{ A } & \multicolumn{4}{|c|}{$1-1$} & $2 m$ & \multicolumn{3}{|c|}{$1-1$} & $2 m$ \\
\hline & \multicolumn{2}{|l|}{ AA } & \multicolumn{4}{|c|}{$1-1-1-1-1$} & $5 m$ & \multicolumn{3}{|c|}{0} & $0 m$ \\
\hline & \multicolumn{2}{|l|}{$\mathrm{L}$} & \multicolumn{4}{|c|}{0} & $0 \mathrm{~m}$ & \multicolumn{3}{|c|}{$1-1-2$} & $4 m$ \\
\hline & \multicolumn{2}{|l|}{ Lr } & \multicolumn{4}{|c|}{$2-3$} & $5 m$ & \multicolumn{3}{|c|}{$2-3-2-3$} & $10 \mathrm{~m}$ \\
\hline & \multicolumn{2}{|l|}{$S$} & \multicolumn{4}{|c|}{ 1-1-1-1-1-1-1-1-1 } & $0 \mathrm{~m}$ & \multicolumn{3}{|c|}{0} & $0 m$ \\
\hline & \multicolumn{2}{|c|}{ D más realizados } & \multicolumn{4}{|c|}{ S - F - AA } & $=$ & \multicolumn{3}{|c|}{$F-L r-L$} & $=$ \\
\hline & \multicolumn{6}{|c|}{ Sumatoria de distancias recorridos } & \multicolumn{4}{|l|}{$32 \mathrm{~m}$} & $30 \mathrm{~m}$ \\
\hline & $\begin{array}{c}\text { PUNTOS DEL } \\
\text { GAME }\end{array}$ & $1^{\text {er }} \mathrm{P}$ & $2^{\text {do }} \mathrm{P}$ & $3^{\text {er }} p$ & $4^{\text {to }} \mathrm{P}$ & $5^{\text {to }} \mathrm{P}$ & $6^{\text {to }} \mathrm{P}$ & $7^{\mathrm{mo}} \mathrm{P}$ & $8^{\text {vo }} \mathrm{P}$ & $9^{\text {no }} \mathrm{P}$ & $10^{\text {mo }} \mathrm{P}$ \\
\hline & T trabajo & $2,73^{\prime \prime}$ & $4,17^{\prime \prime}$ & $2,57^{\prime \prime}$ & $3,20^{\prime \prime}$ & $8,41^{\prime \prime}$ & $7,64 "$ & -- & -- & -- & -- \\
\hline & T descanso & $20,34 "$ & $28,96 "$ & $27,04^{\prime \prime}$ & $22,39^{\prime \prime}$ & $23,70^{\prime \prime}$ & $\rightarrow$ & - & -- & -- & -- \\
\hline & \multicolumn{6}{|c|}{ Tiempo en el cambio de lado } & $103 "$ & & & & \\
\hline
\end{tabular}

Tabla 10. 


\begin{tabular}{|c|c|c|c|c|c|c|c|c|c|c|c|}
\hline \multirow{13}{*}{ 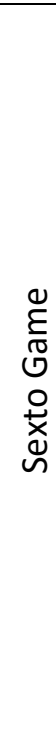 } & \multicolumn{2}{|c|}{ DESPLAZAMIENTOS } & \multicolumn{4}{|c|}{ D CIBULKOVA } & Total & \multicolumn{3}{|c|}{ LI NA } & Total \\
\hline & \multicolumn{2}{|c|}{$\mathrm{F}$} & \multicolumn{4}{|c|}{$\begin{array}{c}1-2-4-2-4-6-3-2-3-1-3-6-1- \\
1-4-2-1-4-2-1-1-1-3\end{array}$} & $58 \mathrm{~m}$ & \multicolumn{3}{|c|}{$3-1-2-1-3-3-1-2-4-2-2$} & $24 m$ \\
\hline & \multicolumn{2}{|l|}{ A } & \multicolumn{4}{|c|}{$1-1$} & $2 m$ & \multicolumn{3}{|c|}{1} & $0 m$ \\
\hline & \multicolumn{2}{|l|}{ AA } & \multicolumn{4}{|c|}{0} & $0 m$ & \multicolumn{3}{|c|}{$1-1-1-1$} & $4 m$ \\
\hline & \multicolumn{2}{|l|}{$\mathrm{L}$} & \multicolumn{4}{|c|}{$1-2$} & $3 m$ & \multicolumn{3}{|c|}{1} & $1 \mathrm{~m}$ \\
\hline & \multicolumn{2}{|l|}{$\mathrm{Lr}$} & \multicolumn{4}{|c|}{$2-3-2-2-1-2-3-2-2$} & $19 \mathrm{~m}$ & \multicolumn{3}{|c|}{$2-1-1-2-2-1-2-1-1$} & $13 m$ \\
\hline & \multicolumn{2}{|l|}{$S$} & \multicolumn{4}{|c|}{0} & $0 m$ & \multicolumn{3}{|c|}{ 1-1-1-1-1-1-1-1-1 } & $0 m$ \\
\hline & \multicolumn{2}{|c|}{ D más realizados } & \multicolumn{4}{|c|}{$\mathbf{F}-\mathbf{L r}-\mathbf{L}$} & $=$ & \multicolumn{3}{|c|}{$F-L r-S$} & $=$ \\
\hline & \multicolumn{6}{|c|}{ Sumatoria de distancias recorridos } & $72 m$ & & & & $42 m$ \\
\hline & $\begin{array}{l}\text { PUNTOS DEL } \\
\text { GAME }\end{array}$ & $1^{\text {er }} P$ & $2^{\text {do }} \mathrm{P}$ & $3^{\text {er }} \mathrm{P}$ & $4^{\text {to }} \mathrm{P}$ & $5^{\text {to }} \mathrm{P}$ & $6^{\text {to }} P$ & $7^{\mathrm{mo}} \mathrm{P}$ & $8^{\text {vo }} \mathrm{P}$ & $9^{\text {no }} P$ & $10^{\text {mo }} \mathrm{P}$ \\
\hline & $\mathrm{T}$ trabajo & $28,78^{\prime \prime}$ & $10,42^{\prime \prime}$ & $5,55^{\prime \prime}$ & $2,82^{\prime \prime}$ & $3,20^{\prime \prime}$ & -- & -- & -- & -- & -- \\
\hline & T descanso & $40,84^{\prime \prime}$ & $27,26^{\prime \prime}$ & $29,68^{\prime \prime}$ & $23,65^{\prime \prime}$ & $\rightarrow$ & -- & -- & -- & -- & -- \\
\hline & \multicolumn{6}{|c|}{\begin{tabular}{l|l} 
Tiempo en el cambio de servicio & $39,40^{\prime \prime}$
\end{tabular}} & & & & & \\
\hline
\end{tabular}

Tabla 11.

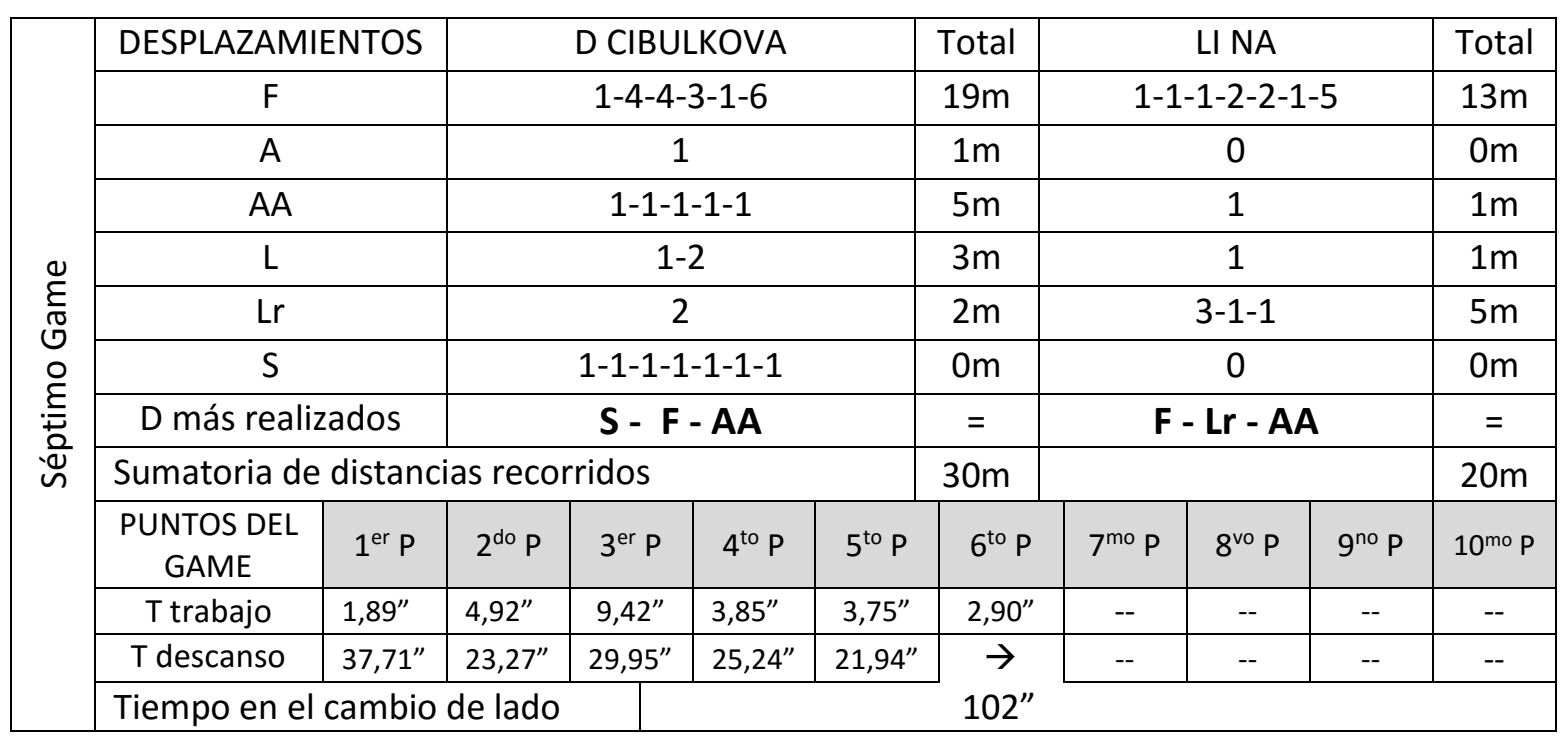

Tabla 12. 


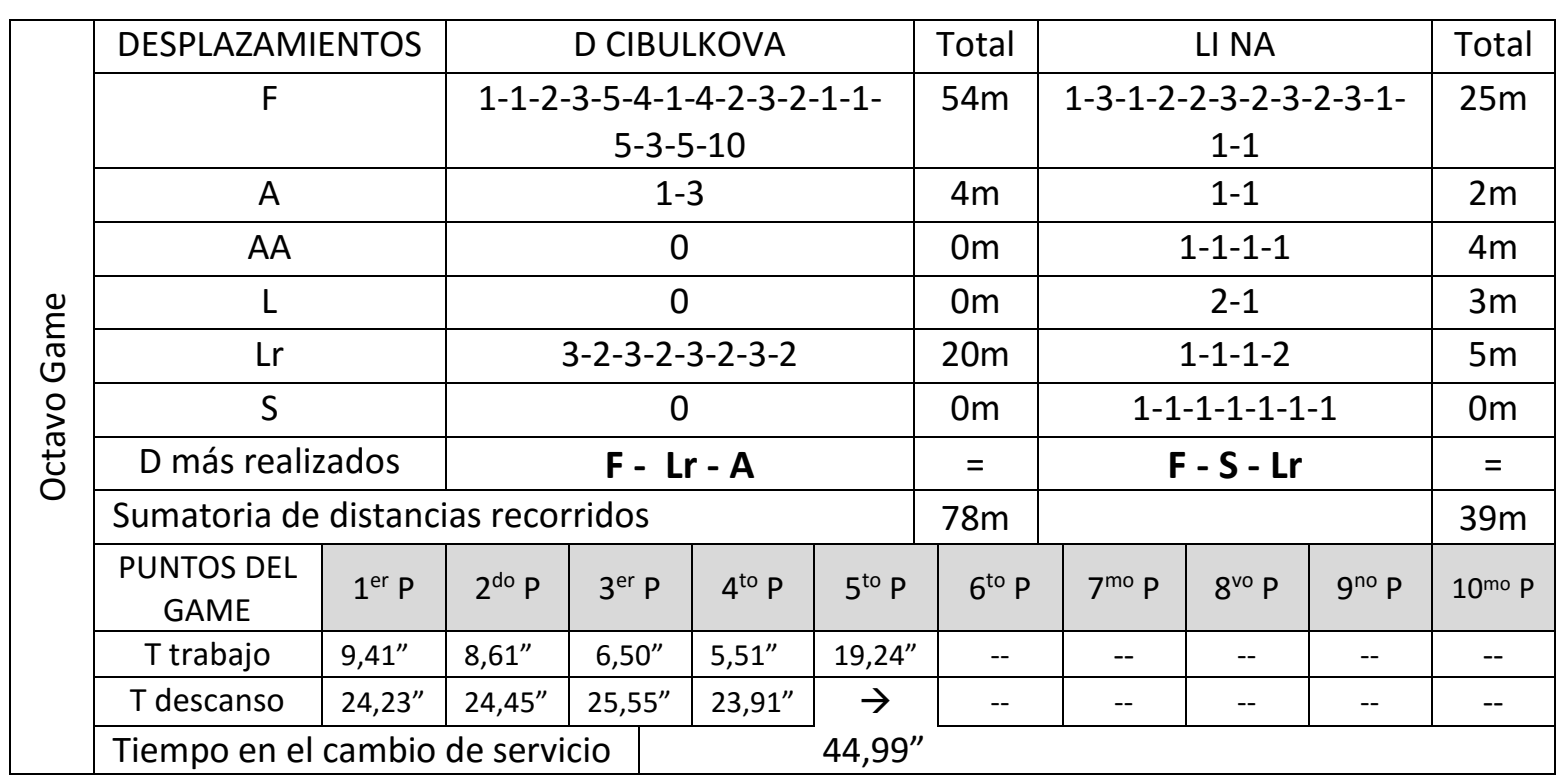

Tabla 13.

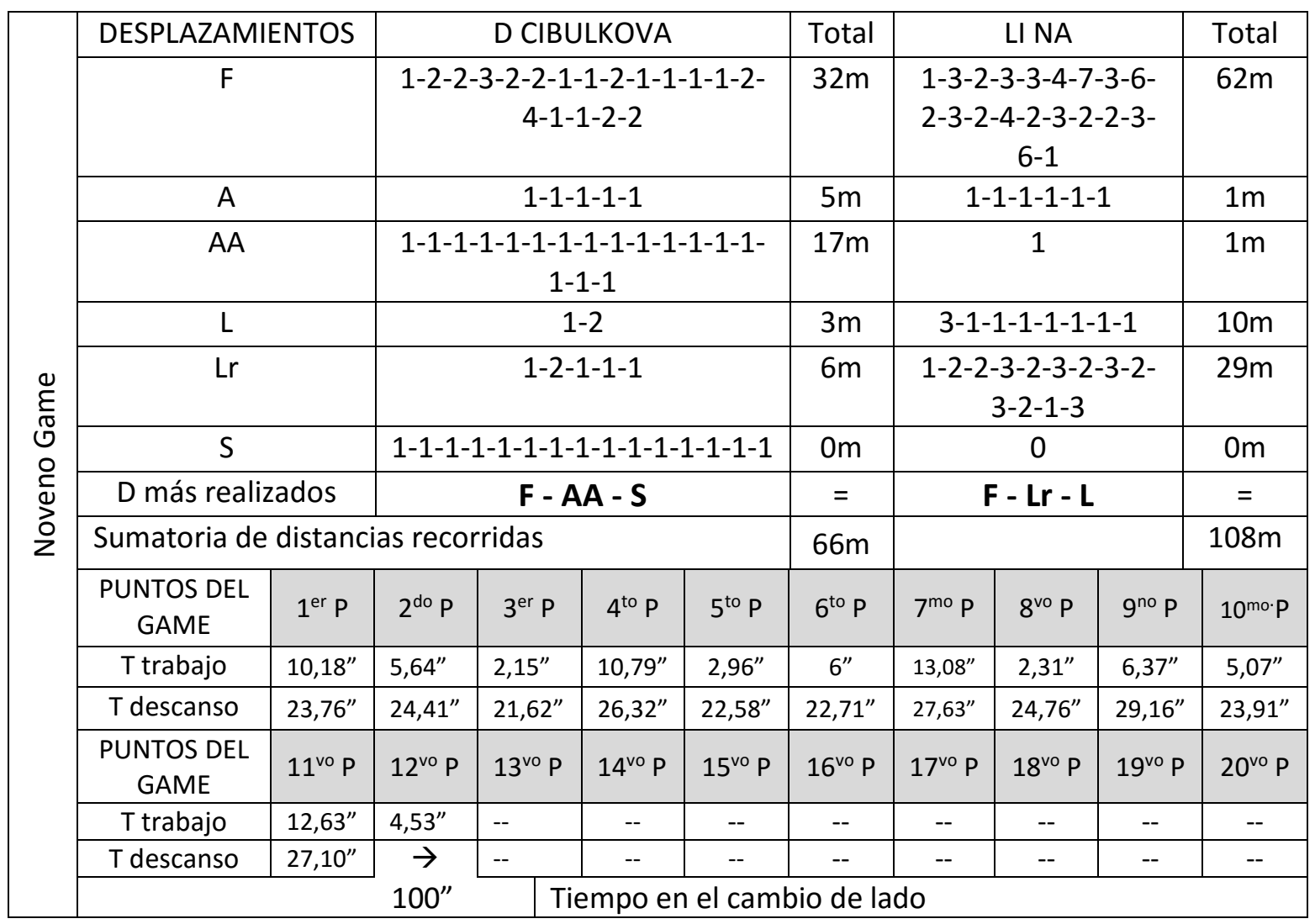

\footnotetext{
Tabla 14.
} 


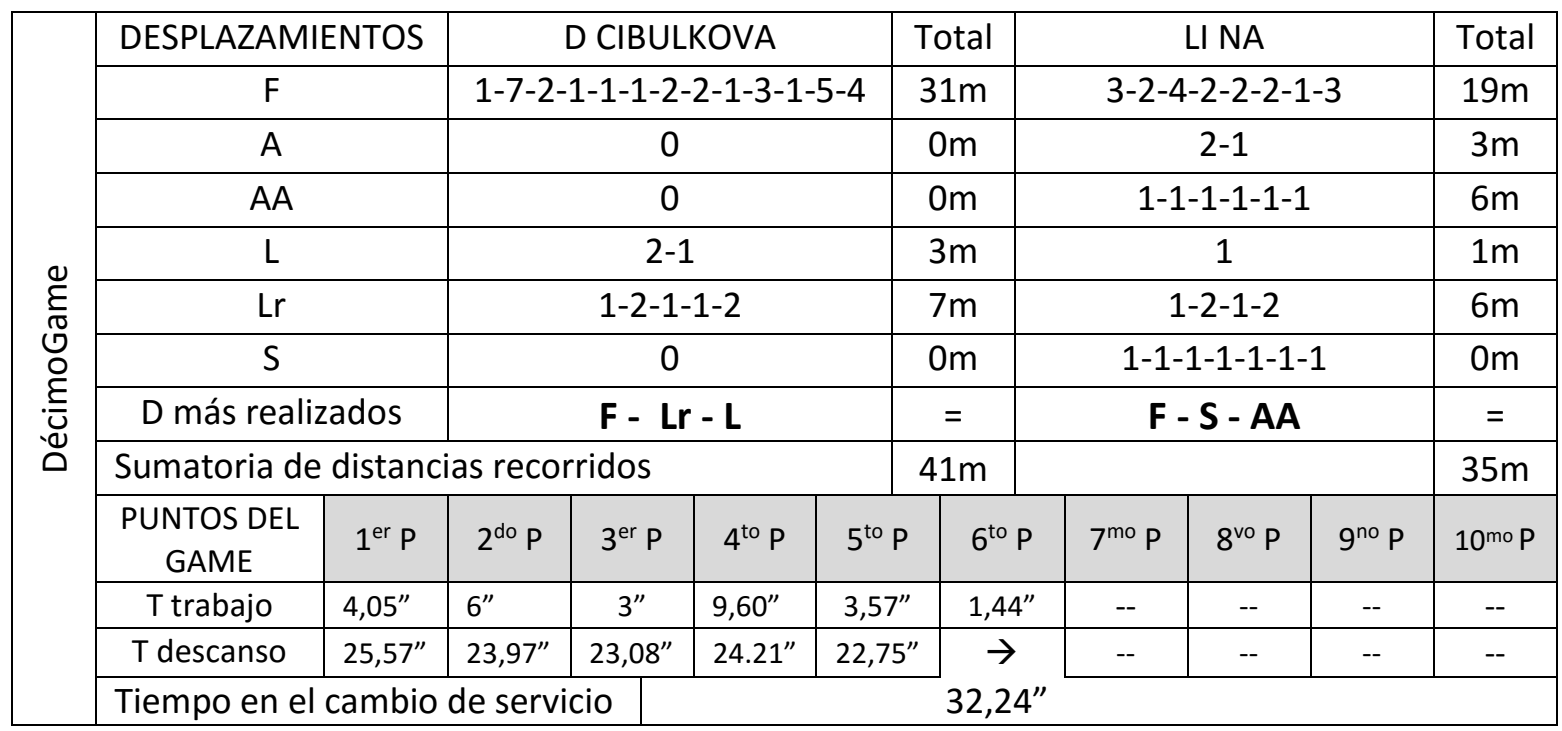

Tabla 15.

\begin{tabular}{|c|c|c|c|c|c|c|c|c|c|c|c|}
\hline \multirow{13}{*}{ 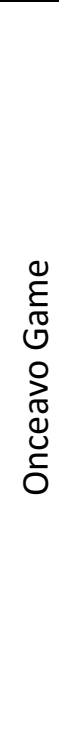 } & \multicolumn{2}{|c|}{ DESPLAZAMIENTOS } & \multicolumn{4}{|c|}{ D CIBULKOVA } & Total & \multicolumn{3}{|c|}{ LI NA } & Total \\
\hline & \multicolumn{2}{|l|}{$\mathrm{F}$} & \multicolumn{4}{|c|}{$\begin{array}{c}2-2-1-3-2-3-2-1-1-6-3-1-1- \\
3-3-1-5-5\end{array}$} & $45 m$ & \multicolumn{3}{|c|}{$\begin{array}{c}1-1-2-2-2-4-2-3-2-2-3- \\
4-1-3\end{array}$} & $32 \mathrm{~m}$ \\
\hline & \multicolumn{2}{|l|}{$A$} & \multicolumn{4}{|c|}{ 1-2-2-1 } & $6 m$ & \multicolumn{3}{|c|}{$2-1$} & $3 m$ \\
\hline & \multicolumn{2}{|l|}{ AA } & \multicolumn{4}{|c|}{ 1-1-1-1 } & $4 \mathrm{~m}$ & \multicolumn{3}{|c|}{1} & $1 \mathrm{~m}$ \\
\hline & \multicolumn{2}{|l|}{$\mathrm{L}$} & \multicolumn{4}{|c|}{$1-1$} & $2 m$ & \multicolumn{3}{|c|}{$1-1-1-1$} & $4 m$ \\
\hline & \multicolumn{2}{|l|}{$\mathrm{Lr}$} & \multicolumn{4}{|c|}{$1-1-3-1-2-1-2-2-2-2$} & $17 \mathrm{~m}$ & \multicolumn{3}{|c|}{$2-1-2-1-2-2-1-3-2-3-2$} & $21 \mathrm{~m}$ \\
\hline & \multicolumn{2}{|l|}{$S$} & \multicolumn{4}{|c|}{ 1-1-1-1-1-1-1 } & $0 \mathrm{~m}$ & \multicolumn{3}{|c|}{0} & $0 \mathrm{~m}$ \\
\hline & \multicolumn{2}{|c|}{ D más realizados } & \multicolumn{4}{|c|}{$F-L r-S$} & $=$ & \multicolumn{3}{|c|}{$F-L r-L$} & $=$ \\
\hline & \multicolumn{6}{|c|}{ Sumatoria de distancias recorridos } & $74 \mathrm{~m}$ & & & & $61 \mathrm{~m}$ \\
\hline & $\begin{array}{l}\text { PUNTOS DEL } \\
\text { GAME }\end{array}$ & $1^{\text {er }} \mathrm{P}$ & $2^{\text {do }} \mathrm{P}$ & $3^{\text {er }} \mathrm{P}$ & $4^{\text {to }} \mathrm{P}$ & $5^{\text {to }} \mathrm{P}$ & $6^{\text {to }} \mathrm{P}$ & $7^{\text {mo }} \mathrm{P}$ & $8^{\mathrm{vo}} \mathrm{P}$ & $9^{\text {no }} \mathrm{p}$ & $10^{\text {mo }} \mathrm{P}$ \\
\hline & T trabajo & $30,58^{\prime \prime}$ & $2,59^{\prime \prime}$ & $1,20^{\prime \prime}$ & $23,16 "$ & $3,58^{\prime \prime}$ & -- & -- & -- & -- & -- \\
\hline & T descanso & $33,76^{\prime \prime}$ & $33,59 "$ & $15^{\prime \prime}$ & $44,47^{\prime \prime}$ & $\rightarrow$ & -- & -- & -- & -- & -- \\
\hline & \multicolumn{6}{|c|}{\begin{tabular}{|l|l} 
Tiempo en el cambio de lado & $102^{\prime \prime}$
\end{tabular}} & & & & & \\
\hline
\end{tabular}

Tabla 16. 


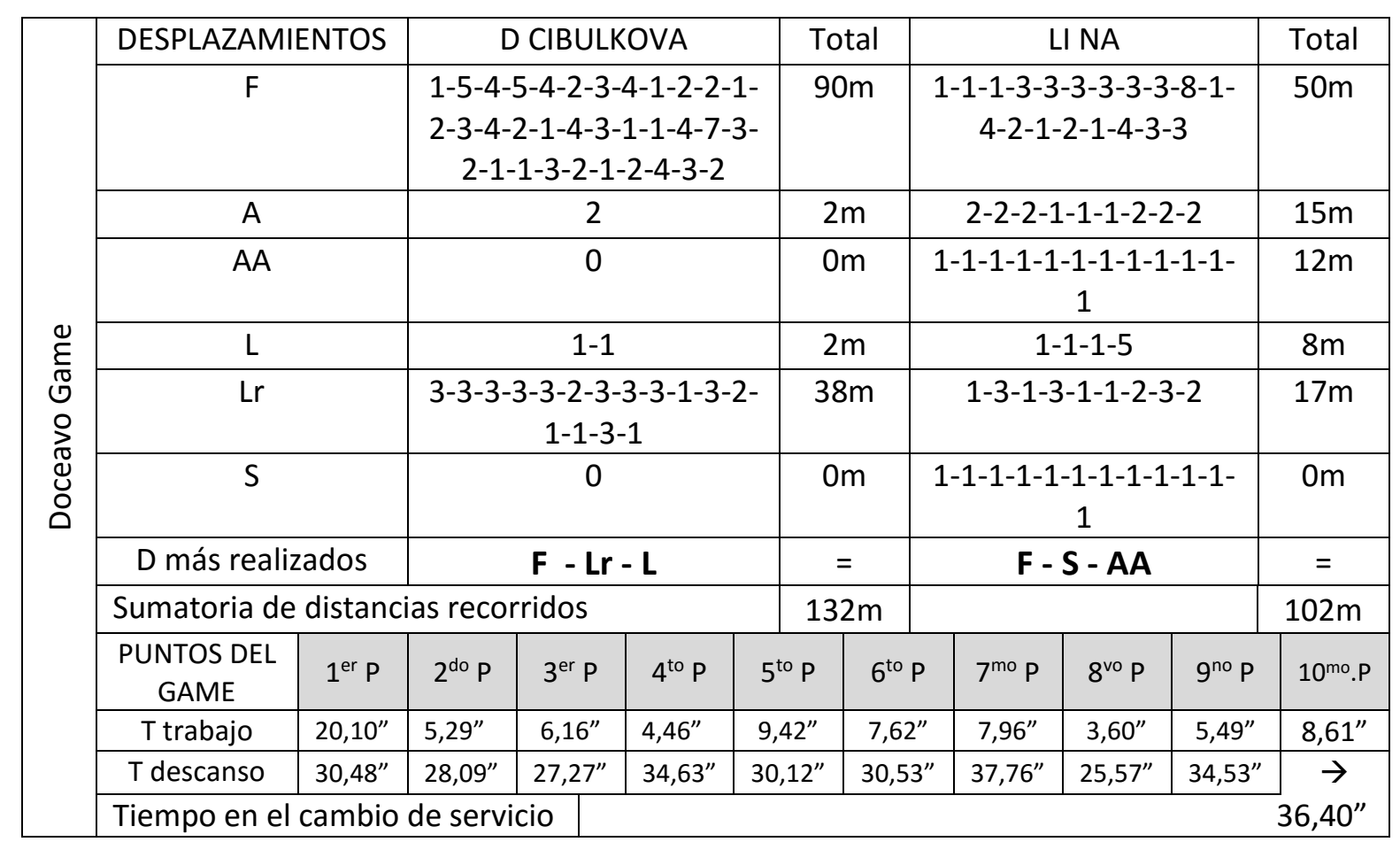

Tabla 17.

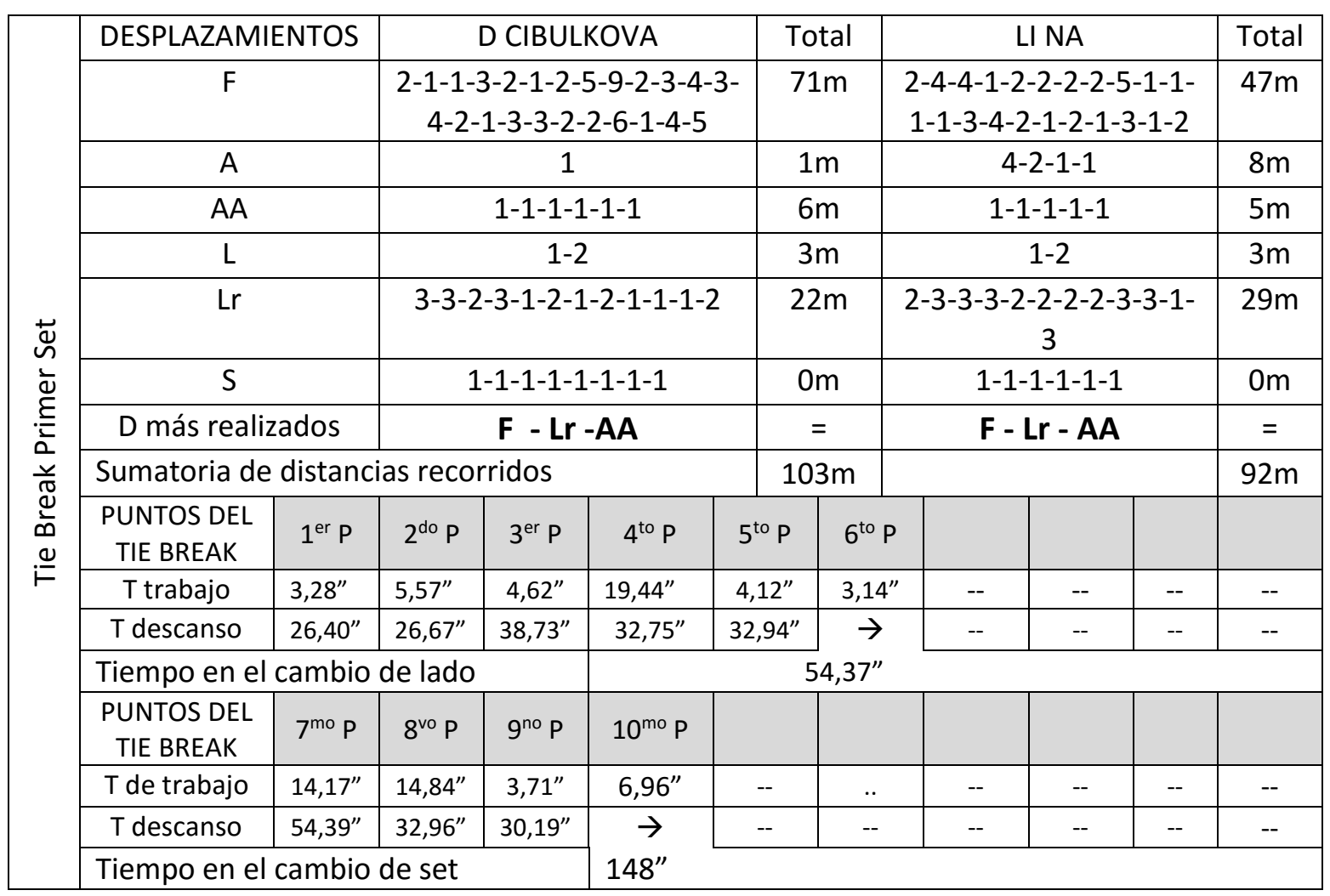

\section{Tabla 18}




\begin{tabular}{|c|c|c|c|c|c|c|c|}
\hline \multicolumn{8}{|c|}{$\begin{array}{l}\text { TIPOS DE DESPLAZAMIENTOS, LOS MÁS UTILIZADOS Y SUS DISTANCIAS. PRIMER SET DEL PARTIDO DE FINAL DEL GRAND SLAM AUSTRALIAN OPEN } \\
\text { 2014, ENTRE DOMINIKA CIBULKOVA Y LI NA.SUPERFICIE: DURA. }\end{array}$} \\
\hline $\begin{array}{c}\text { TIPOS DE } \\
\text { DESPLAZAMIENTOS }\end{array}$ & \multicolumn{4}{|c|}{ TOTAL POR SET } & $\begin{array}{l}\text { DISTANCIA MÁS } \\
\text { RECORRIDA }\end{array}$ & $\begin{array}{l}\text { SUMATORIA DE DISTANCIAS } \\
\text { RECORRIDAS POR SET }\end{array}$ & $\begin{array}{c}\text { TOTAL } \\
\text { RECORRIDO }\end{array}$ \\
\hline \multirow{8}{*}{$\begin{array}{l}\text { Desplazamientos de } \\
\text { frente }\end{array}$} & $1 \mathrm{~m}$ & \multicolumn{3}{|c|}{$\begin{array}{c}* * * * * * * * * * * * * * * * * * * * * * * * * * * * * * * * * * * * * * \\
* * * * * * * * * * * * * * * * * * * * * * * * * * * * * * * * * * * * * * * \\
* * * * * * * * * * * * * * * * * * * * * * * * * * * * * * * * * * * * * * * \\
* * * * * *\end{array}$} & \multirow{8}{*}{1 Metro } & \multirow{3}{*}{$\begin{array}{l}1 \mathrm{~m}=123 \text { veces }=123 \mathrm{~m} \\
2 \mathrm{~m}=107 \text { veces }=214 \mathrm{~m} \\
3 \mathrm{~m}=79 \text { veces }=237 \mathrm{~m} \\
4 \mathrm{~m}=39 \text { veces }=156 \mathrm{~m} \\
5 \mathrm{~m}=17 \text { veces }=85 \mathrm{~m} \\
6 \mathrm{~m}=8 \text { veces }=48 \mathrm{~m} \\
7 \mathrm{~m}=3 \text { veces }=21 \mathrm{~m} \\
8 \mathrm{~m}=1 \text { vez }=8 \mathrm{~m} \\
9 \mathrm{~m}=1 \text { vez }=9 \mathrm{~m} \\
10 \mathrm{~m}=1 \text { vez }=10 \mathrm{~m}\end{array}$} & \multirow{12}{*}{$1.553 \mathrm{~m}}$. \\
\hline & $2 m$ & $\begin{array}{r}* * * * * * * * * * \\
* * * * * * * * * * \\
* * * * *\end{array}$ & $\begin{array}{l}* * * * \\
* * * * \\
* * * *\end{array}$ & $\begin{array}{l}* * * * * * * * \\
* * * * * * * * \\
* *\end{array}$ & & & \\
\hline & $3 m$ & $\begin{array}{l}* * * * * * * * * * \\
* * * * * * * * * *\end{array}$ & $\begin{array}{l}* * * * \\
* * * * \\
*\end{array}$ & $\begin{array}{l}* * * * * * * * \\
* * * * * * * *\end{array}$ & & & \\
\hline & $4 m$ & $* * * * * * * * * *$ & $* * * *$ & $* * * * * * * *$ & & \multirow{5}{*}{ 911m. } & \\
\hline & $5 m$ & & & & & & \\
\hline & $6 m$ & & $* * * * *$ & & & & \\
\hline & $7 m$ & $* * *$ & $8 \mathrm{~m}$ & $*$ & & & \\
\hline & $9 m$ & $*$ & $10 \mathrm{~m}$ & $*$ & & & \\
\hline \multirow{3}{*}{$\begin{array}{l}\text { Desplazamientos hacia } \\
\text { atrás de ejecución }\end{array}$} & $1 \mathrm{~m}$ & $* * * * * *$ & $* * * * *$ & $* * * *$ & \multirow{3}{*}{1 Metro } & \multirow{2}{*}{$\begin{array}{l}1 \mathrm{~m}=46 \text { veces }=46 \mathrm{~m} \\
2 \mathrm{~m}=17 \text { veces }=34 \mathrm{~m} \\
3 \mathrm{~m}=2 \text { veces }=6 \mathrm{~m} \\
4 \mathrm{~m}=1 \text { vez }=4 \mathrm{~m}\end{array}$} & \\
\hline & $2 m$ & \multicolumn{3}{|c|}{$* * * * * * * * * * * * * * * * * *$} & & & \\
\hline & $3 m$ & $* *$ & $4 \mathrm{~m}$ & $*$ & & $\begin{array}{r}4 \mathrm{~m}=1 \mathrm{vez}=4 \mathrm{~m} \\
90 \mathrm{~m}\end{array}$ & \\
\hline $\begin{array}{l}\text { Desplazamientos hacia } \\
\text { atrás de recuperación }\end{array}$ & $1 \mathrm{~m}$ & \multicolumn{3}{|c|}{$\begin{array}{c}* * * * * * * * * * * * * * * * * * * * * * * * * * * * * * * * * * * * * * * \\
* * * * * * * * * * * * * * * * * * * * * * * * * * * * * * * * * * * * * * * \\
* * * * * * * * * * * * * * * * * * * * * * * * * * * * * * * * * * * * * * * \\
\\
* * * * * * * \\
\\
* * * * * * *\end{array}$} & 1 Metro & $1 \mathrm{~m}=131$ veces $=131 \mathrm{~m}$ & \\
\hline
\end{tabular}

Tabla 19. 


\begin{tabular}{|c|c|c|c|c|c|}
\hline \multicolumn{6}{|c|}{$\begin{array}{l}\text { TIPOS DE DESPLAZAMIENTOS, LOS MÁS UTILIZADOS Y SUS DISTANCIAS. PRIMER SET DEL PARTIDO DE FINAL DEL GRAND SLAM AUSTRALIAN OPEN } \\
\text { 2014, ENTRE DOMINIKA CIBULKOVA Y LI NA. SUPERFICIE: DURA. }\end{array}$} \\
\hline $\begin{array}{c}\text { TIPOS DE } \\
\text { DESPLAZAMIENTOS }\end{array}$ & \multicolumn{2}{|c|}{ TOTAL POR SET } & $\begin{array}{l}\text { DISTANCIA MÁS } \\
\text { RECORRIDA }\end{array}$ & $\begin{array}{l}\text { SUMATORIA DE DISTANCIAS } \\
\text { RECORRIDAS POR SET }\end{array}$ & $\begin{array}{c}\text { TOTAL } \\
\text { RECORRIDO }\end{array}$ \\
\hline \multirow{4}{*}{$\begin{array}{l}\text { Desplazamientos } \\
\text { laterales de ejecución }\end{array}$} & $1 \mathrm{~m}$ & $\begin{array}{l}* * * * * * * * * * * * * * * * * * * * * * * * * * * * * * * * * * * * * \\
* * * * * * * * *\end{array}$ & \multirow{4}{*}{1 Metro } & \multirow{3}{*}{$\begin{array}{l}1 \mathrm{~m}=45 \text { veces }=45 \mathrm{~m} \\
2 \mathrm{~m}=17 \text { veces }=17 \mathrm{~m} \\
3 \mathrm{~m}=1 \text { vez }=3 \mathrm{~m} \\
5 \mathrm{~m}=1 \text { vez }=5 \mathrm{~m}\end{array}$} & \multirow{8}{*}{$\uparrow$} \\
\hline & $2 m$ & $* * * * * * * * * * * * * * * * *$ & & & \\
\hline & $3 m$ & $*$ & & & \\
\hline & $5 m$ & $*$ & & $64 \mathrm{~m}$. & \\
\hline \multirow{3}{*}{$\begin{array}{l}\text { Desplazamientos } \\
\text { laterales de } \\
\text { recuperación. }\end{array}$} & $1 \mathrm{~m}$ & $\begin{array}{l}* * * * * * * * * * * * * * * * * * * * * * * * * * * * * * * * * * * * * \\
\quad * * * * * * * * * * * * * * * * * * * * *\end{array}$ & \multirow{3}{*}{2 Metros } & \multirow{2}{*}{$\begin{array}{l}1 \mathrm{~m}=60 \text { veces }=60 \mathrm{~m} \\
2 \mathrm{~m}=84 \text { veces }=168 \mathrm{~m} \\
3 \mathrm{~m}=43 \text { veces }=129 \mathrm{~m}\end{array}$} & \\
\hline & $2 m$ & $\begin{array}{c}* * * * * * * * * * * * * * * * * * * * * * * * * * * * * * * * * * * * * \\
* * * * * * * * * * * * * * * * * * * * * * * * * * * * * * * * * * * * * \\
* * * * * * * *\end{array}$ & & & \\
\hline & $3 m$ & $\begin{array}{l}* * * * * * * * * * * * * * * * * * * * * * * * * * * * * * * * * * * * * \\
\quad * * * * * * * * * * * * * * * * * * * * *\end{array}$ & & $357 \mathrm{~m}$. & \\
\hline $\begin{array}{l}\text { Desplazamientos hacia } \\
\text { arriba o saltos }\end{array}$ & \multicolumn{2}{|c|}{$\begin{array}{c}* * * * * * * * * * * * * * * * * * * * * * * * * * * * * * * * * * * * * * * * * * \\
* * * * * * * * * * * * * * * * * * * * * * * * * * * * * * * * * * * * * * * * \\
* * * * * * * * * * * * * * * * * * * * * * * * * * * * * * * * * * * *\end{array}$} & \multicolumn{2}{|c|}{ Saltos en servicio $=122$ veces } & \\
\hline
\end{tabular}

Tabla 20

\begin{tabular}{|c|c|c|c|c|c|c|}
\hline \multicolumn{7}{|c|}{$\begin{array}{l}\text { PORCENTAJES DE USO DE LOS DIFERENTES TIPOS DE DESPLAZAMIENTOS EN EL PRIMER SET DEL PARTIDO DE FINAL DEL GRAND SLAM AUSTRALIA } \\
\text { OPEN 2014, ENTRE DOMINIKA CIBULKOVA Y LI NA. SUPERFICIE: DURA. }\end{array}$} \\
\hline & D HACIA ADELANTE & $\begin{array}{l}\text { D HACIA ATRÁS } \\
\text { DE EJECUCIÓN }\end{array}$ & $\begin{array}{l}\text { D HACIA ATRÁS DE } \\
\text { RECUPERACIÓN }\end{array}$ & $\begin{array}{l}\text { D LATERALES DE } \\
\text { EJECUCIÓN }\end{array}$ & $\begin{array}{l}\text { D LATERALES DE } \\
\text { RECUPERACIÓN }\end{array}$ & D HACIA ARRIBA \\
\hline NÚMERO DE VECES & $40,70 \%$ & $7,08 \%$ & $12,13 \%$ & $6,87 \%$ & $20,08 \%$ & $13,10 \%$ \\
\hline DISTANCIA & $58,66 \%$ & $5,79 \%$ & $8,43 \%$ & $4,12 \%$ & $22,98 \%$ & \\
\hline
\end{tabular}

Tabla 21. 


\begin{tabular}{|c|c|c|c|c|}
\hline \multicolumn{5}{|c|}{$\begin{array}{l}\text { TIEMPOS GLOBALES DE LOS MOMENTOS DE TRABAJO Y PAUSA DEL PRIMER SET DEL PARTIDO } \\
\text { ENTRE DOMINIKA CIBULKOVA Y LI NA, EN LA FINAL DEL AUSTRALIAN OPEN DE TENIS } 2014\end{array}$} \\
\hline TIEMPOS & \multicolumn{2}{|c|}{$\begin{array}{l}\text { SUMATORIA DE } \\
\text { TIEMPOS POR GAME }\end{array}$} & $\begin{array}{l}\text { SUMATORIA DE TIEMPOS } \\
\text { POR SET }\end{array}$ & $\begin{array}{l}\text { RELACIÓN TIEMPO } \\
\text { DE TRABAJO-PAUSA }\end{array}$ \\
\hline \multirow{13}{*}{ TIEMPO DE TRABAJO } & $1^{\mathrm{er}}$ Game & $61,49^{\prime \prime}$ & \multirow{13}{*}{$\begin{array}{l}693.9 " \text { tiempo real de } \\
\text { trabajo }\end{array}$} & \multirow{26}{*}{$\begin{array}{l}1 " \text { de trabajo por } \\
4,72^{\prime \prime} \text { de pausa }\end{array}$} \\
\hline & $2^{\text {do }}$ Game & $24,88^{\prime \prime}$ & & \\
\hline & $3^{\text {er }}$ Game & $86,51^{\prime \prime}$ & & \\
\hline & $4^{\text {to }}$ Game & $36,29^{\prime \prime}$ & & \\
\hline & $5^{\text {to }}$ Game & $28,72^{\prime \prime}$ & & \\
\hline & $6^{\text {to }}$ Game & $50,77^{\prime \prime}$ & & \\
\hline & $7^{\text {to }}$ Game & $26,73^{\prime \prime}$ & & \\
\hline & $8^{\text {vo }}$ Game & $49,27 "$ & & \\
\hline & $9^{\text {no }}$ Game & $81,71^{\prime \prime}$ & & \\
\hline & $10^{\mathrm{mo}}$ Game & $27,66^{\prime \prime}$ & & \\
\hline & $11^{\text {vo }}$ Game & $61,11^{\prime \prime}$ & & \\
\hline & $12^{\text {vo }}$ Game & $78,71^{\prime \prime}$ & & \\
\hline & Tie Break & $80,05^{\prime \prime}$ & & \\
\hline \multirow{13}{*}{ TIEMPO DE DESCANSO } & $1^{\text {er }}$ Game & $277,61^{\prime \prime}$ & \multirow{13}{*}{$\begin{array}{l}\text { 3.280,15" tiempo real de } \\
\text { Descanso }\end{array}$} & \\
\hline & $2^{\text {do }}$ Game & $162,08^{\prime \prime}$ & & \\
\hline & $3^{\text {er }}$ Game & $379,94^{\prime \prime}$ & & \\
\hline & $4^{\text {to }}$ Game & $141,71^{\prime \prime}$ & & \\
\hline & $5^{\text {to }}$ Game & $225,43^{\prime \prime}$ & & \\
\hline & $6^{\text {to }}$ Game & $160,83^{\prime \prime}$ & & \\
\hline & $7^{\text {to }}$ Game & $240,11^{\prime \prime}$ & & \\
\hline & $8^{\text {vo }}$ Game & $143,13^{\prime \prime}$ & & \\
\hline & $9^{\text {no }}$ Game & $373,95^{\prime \prime}$ & & \\
\hline & $10^{\mathrm{mo}}$ Game & $151,82^{\prime \prime}$ & & \\
\hline & $11^{\text {vo }}$ Game & $228,82^{\prime \prime}$ & & \\
\hline & $12^{\text {vo }}$ Game & $315,38^{\prime \prime}$ & & \\
\hline & Tie Break & $479,34^{\prime \prime}$ & & \\
\hline
\end{tabular}

Tabla 22. 


\begin{tabular}{|c|c|c|c|}
\hline \multicolumn{4}{|c|}{$\begin{array}{l}\text { PROMEDIO DE LOS MOMENTOS ESPECÍFICOS DE TRABAJO Y PAUSA DEL PRIMER SET DEL PARTIDO } \\
\text { ENTRE DOMINIKA CIBULKOVA Y LI NA, EN LA FINAL DEL AUSTRALIA OPEN DE TENIS } 2014\end{array}$} \\
\hline \multicolumn{2}{|c|}{ PROMEDIOS DE TRABAJO } & \multicolumn{2}{|c|}{ PROMEDIOS DE PAUSA } \\
\hline $1^{\text {er }}$ Game & $6,14^{\prime \prime}$ & $1^{\text {er }}$ Game & $24,53^{\prime \prime}$ \\
\hline $2^{\text {do }}$ Game & $4,14^{\prime \prime}$ & $2^{\text {do }}$ Game & $25,62^{\prime \prime}$ \\
\hline $3^{\text {er }}$ Game & $7,20^{\prime \prime}$ & $3^{\text {er }}$ Game & $25,44^{\prime \prime}$ \\
\hline $4^{\text {to }}$ Game & $7,25^{\prime \prime}$ & $4^{\text {to }}$ Game & $25,85^{\prime \prime}$ \\
\hline $5^{\text {to }}$ Game & $4,78^{\prime \prime}$ & $5^{\text {to }}$ Game & $24,48^{\prime \prime}$ \\
\hline $6^{\text {to }}$ Game & $12,69^{\prime \prime}$ & $6^{\text {to }}$ Game & $30,35^{\prime \prime}$ \\
\hline $7^{\mathrm{mo}}$ Game & $4,45^{\prime \prime}$ & $7^{\mathrm{mo}}$ Game & $23,01^{\prime \prime}$ \\
\hline $8^{\text {vo }}$ Game & $9,85^{\prime \prime}$ & $8^{\text {vo }}$ Game & $19,68^{\prime \prime}$ \\
\hline $9^{\text {no }}$ Game & $6,80^{\prime \prime}$ & $9^{\text {no }}$ Game & $24,90^{\prime \prime}$ \\
\hline $10^{\mathrm{mo}}$ Game & $5,53^{\prime \prime}$ & $10^{\mathrm{mo}}$ Game & $23,91^{\prime \prime}$ \\
\hline $11^{\text {vo }}$ Game & $12,22^{\prime \prime}$ & $11^{\text {vo }}$ Game & $21,70^{\prime \prime}$ \\
\hline $12^{\text {vo }}$ Game & $7,80^{\prime \prime}$ & $12^{\text {vo }}$ Game & $30,99 "$ \\
\hline Tie Break & $8^{\prime \prime}$ & Tie Break & $36,6^{\prime \prime}$ \\
\hline \multicolumn{2}{|c|}{ Promedio total } & \multicolumn{2}{|c|}{ Promedio total } \\
\hline \multicolumn{2}{|c|}{$7,45^{\prime \prime}$} & \multicolumn{2}{|c|}{$25,92^{\prime \prime}$} \\
\hline \multicolumn{4}{|c|}{ 1" de trabajo por 3,47 " de descanso } \\
\hline
\end{tabular}

Tabla 23.

2. Análisis punto a punto del primer set del partido de tenis entre Roger Federer (Sui) vs Rafael Nadal (Esp), en semifinales del Grand Slam Australian Open 2014, donde Rafael Nadal gana el partido 7-5, 6-3, 6-3. Tipo de superficie de la cancha (dura), clasificación ITF categoría 3 (velocidad media). Ver tablas25 a 42.

- Análisis de los tiempos y tipos de golpes ejecutados dentro de la cancha antes de comenzar el partido (peloteo previo al partido).

Los jugadores empiezan peloteando desde el fondo de la cancha a una intensidad media para entrar en ritmo de juego, pasando la pelota solo por la mitad de la cancha. Al minuto 1,15' R Federer se dirige a tres pasos cerca a la red para hacer voleas, mientras tanto $\mathrm{R}$ Nadal se queda en el fondo lanzándole la pelota para que volee hasta el minuto 1,55'; luego Federer se sitúa sobre la “T” para hacer Smash hasta el minuto 2,10'. Mientras tanto, siguen peloteando en el fondo de la cancha y al minuto 2,40', R Nadal se sitúa a tres pasos cerca a la red para hacer voleas, y Federer se mantiene en el fondo de la cancha lanzándole pelotas a Nadal para que volee hasta el minuto 3,22', para luego 
situarse sobre la "T" y hacer Smash hasta el minuto 3,48'. Luego al minuto 4', los jugadores se sitúan en posición de saque o servicio para hacer dichos golpes hasta el minuto 6,38'; desde este momento hasta el minuto 8', los jugadores se dirigen a sus sillas para descansar y alistarse para comenzar el partido. Ver tabla 24.

\begin{tabular}{|c|c|c|c|c|}
\hline \multicolumn{5}{|c|}{$\begin{array}{c}\text { ANÁLISIS DEL USO DE LOS TIPOS DE GOLPES EN MOMENTO PREVIO AL COMIENZO } \\
\text { DEL PARTIDO(PELOTEO PREVIO AL PARTIDO) }\end{array}$} \\
\hline Tipo de golpe & R Nadal & R Federer & Tiempo promedio & $\%$ \\
\hline $\begin{array}{l}\text { (derecha y revés) Peloteo } \\
\text { de fondo de cancha }\end{array}$ & 160" & $\begin{array}{l}75^{\prime \prime}+30 "+42 "+26 “ \\
=173^{\prime \prime}\end{array}$ & $166,5 "$ & $43 \%$ \\
\hline Volea cerca a la red & $42 "$ & $40 "$ & $41 "$ & $10,62 \%$ \\
\hline Smash sobre la "T" & $26 "$ & $15^{\prime \prime}$ & $20,5 "$ & $5,31 \%$ \\
\hline Servicio o saque & $158 "$ & $158 "$ & $158 "$ & $40,93 \%$ \\
\hline
\end{tabular}

Tabla 24.

\begin{tabular}{|c|c|c|c|c|c|c|c|c|c|c|}
\hline \multirow{13}{*}{ 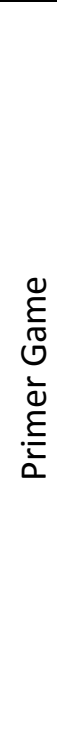 } & $\begin{array}{c}\text { DESPLAZAMIENTO } \\
\mathrm{S}\end{array}$ & \multicolumn{3}{|c|}{ R NADAL } & Total & \multicolumn{4}{|c|}{ R FEDERER } & Total \\
\hline & $\mathrm{F}$ & \multicolumn{3}{|c|}{$1-1-4-6-3-1$} & $16 \mathrm{~m}$ & \multicolumn{3}{|c|}{$5-7$} & & $12 m$ \\
\hline & $A$ & \multicolumn{3}{|c|}{0} & Om & \multicolumn{3}{|c|}{ 3-1 } & & $4 \mathrm{~m}$ \\
\hline & AA & \multicolumn{3}{|c|}{$1-1$} & $2 m$ & \multicolumn{3}{|c|}{$1-1-1$} & & $3 m$ \\
\hline & $\mathrm{L}$ & \multicolumn{3}{|c|}{1} & $1 \mathrm{~m}$ & \multicolumn{3}{|c|}{2} & & $2 \mathrm{~m}$ \\
\hline & $\operatorname{Lr}$ & \multicolumn{3}{|c|}{2} & $2 m$ & \multicolumn{3}{|c|}{$1-1$} & \multicolumn{2}{|r|}{$2 m$} \\
\hline & $S$ & \multicolumn{3}{|c|}{0} & $0 \mathrm{~m}$ & \multicolumn{3}{|c|}{$1-1-1-1-1$} & \multicolumn{2}{|r|}{$5 m$} \\
\hline & D más realizados & \multicolumn{3}{|c|}{ F - AA - Lr } & $=$ & \multicolumn{3}{|c|}{$S-A-F$} & \multicolumn{2}{|r|}{$=$} \\
\hline & \multicolumn{4}{|c|}{ Sumatoria de distancias recorridas } & $21 \mathrm{~m}$ & & & & \multicolumn{2}{|r|}{$28 m$} \\
\hline & PUNTOS DEL GAME & $1^{\mathrm{er}} \mathrm{P}$ & $2^{\text {do }} \mathrm{P}$ & $3^{\text {er }} \mathrm{p}$ & $4^{\text {to }} \mathrm{P}$ & $5^{\text {to }} \mathrm{P}$ & $6^{\text {to }} \mathrm{P}$ & $7^{\mathrm{mo}} \mathrm{P}$ & $8^{\text {vo }} \mathrm{P}$ & $9^{\text {no }} P$ \\
\hline & Tiempo de trabajo & $1,45^{\prime \prime}$ & $6,96^{\prime \prime}$ & $2,64^{\prime \prime}$ & $3,83^{\prime \prime}$ & $3,71^{\prime \prime}$ & -- & -- & -- & -- \\
\hline & Tiempos de descanso & $\begin{array}{l}14,53 \\
\prime \prime\end{array}$ & $\begin{array}{l}17,23 \\
\prime \prime\end{array}$ & $\begin{array}{c}13,16 \\
\prime\end{array}$ & $\begin{array}{c}22,80 \\
\prime \prime\end{array}$ & $\rightarrow$ & -- & -- & -- & -- \\
\hline & \multicolumn{2}{|c|}{ Tiempo en el cambio de lado } & \multicolumn{8}{|c|}{$85^{\prime \prime}$} \\
\hline
\end{tabular}

Tabla 25. 


\begin{tabular}{|c|c|c|c|c|c|c|c|c|c|c|}
\hline \multirow{13}{*}{ 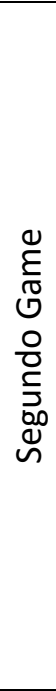 } & DESPLAZAMIENTOS & \multicolumn{3}{|c|}{ R NADAL } & \multicolumn{2}{|c|}{ Total } & \multicolumn{3}{|c|}{ R FEDERER } & Total \\
\hline & $\mathrm{F}$ & \multicolumn{3}{|c|}{2} & $2 m$ & & \multicolumn{3}{|c|}{$1-9-3-1-4-1-4$} & $23 m$ \\
\hline & A & \multicolumn{3}{|c|}{$3-2$} & $5 m$ & & \multicolumn{3}{|c|}{0} & $0 m$ \\
\hline & AA & \multicolumn{3}{|c|}{$1-2$} & $3 m$ & & \multicolumn{3}{|c|}{0} & $0 m$ \\
\hline & $\mathrm{L}$ & \multicolumn{3}{|c|}{$2-2-2$} & $6 m$ & & \multicolumn{3}{|c|}{1} & $1 \mathrm{~m}$ \\
\hline & $\mathrm{Lr}$ & \multicolumn{3}{|c|}{$2-3$} & $5 m$ & & \multicolumn{3}{|c|}{$2-3-1-2$} & $8 m$ \\
\hline & $S$ & \multicolumn{3}{|c|}{$1-1-1-1$} & $0 m$ & & \multicolumn{3}{|c|}{0} & $0 m$ \\
\hline & D más realizados & \multicolumn{3}{|c|}{ S-L-A } & $=$ & & \multicolumn{3}{|c|}{ F- Lr - L } & $=$ \\
\hline & \multicolumn{4}{|c|}{ Sumatoria de distancias recorridas } & \multicolumn{2}{|c|}{$21 \mathrm{~m}$} & & & & \multirow{2}{*}{$\frac{32 \mathrm{~m}}{9^{\text {no }} \mathrm{p}}$} \\
\hline & PUNTOS DEL GAME & $1^{\text {er } P}$ & $2^{\text {do }} \mathrm{P}$ & $3^{\text {er } P}$ & $4^{\text {to }} \mathrm{P}$ & $5^{\text {to }} P$ & $6^{\text {to }} \mathrm{P}$ & $7^{\mathrm{mo}} \mathrm{P}$ & $8^{\text {vo } P}$ & \\
\hline & Tiempo de trabajo & $2,35^{\prime \prime}$ & $9,79^{\prime \prime}$ & $2,91^{\prime \prime}$ & $4,66^{\prime \prime}$ & -- & -- & -- & -- & -- \\
\hline & Tiempos de descanso & $21,31 "$ & $23,94^{\prime \prime}$ & $21,52^{\prime \prime}$ & $\rightarrow$ & -- & -- & -- & -- & -- \\
\hline & Tiempo en el cambio c & servic & & & $29,79 "$ & & & & & \\
\hline
\end{tabular}

Tabla 26.

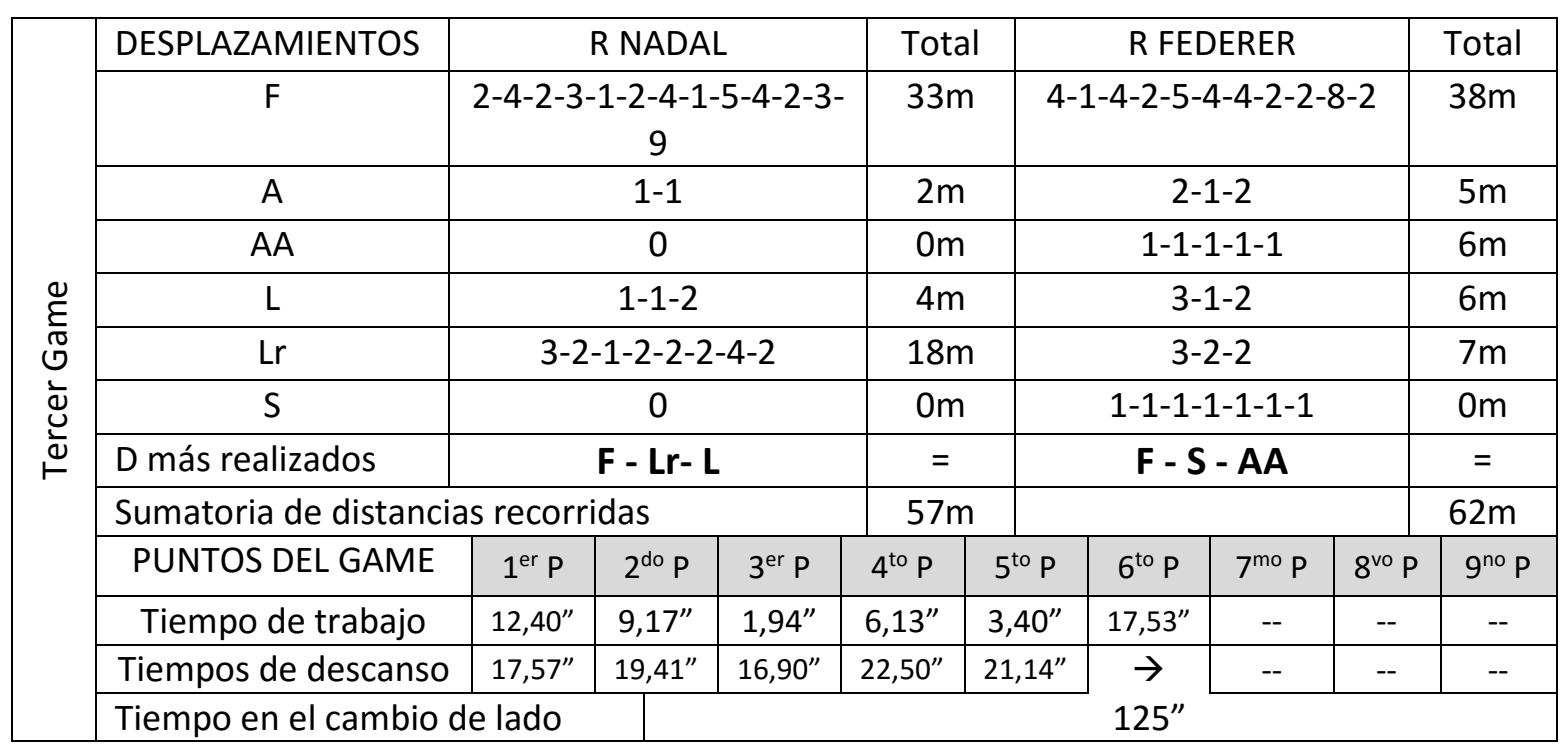

Tabla 27. 


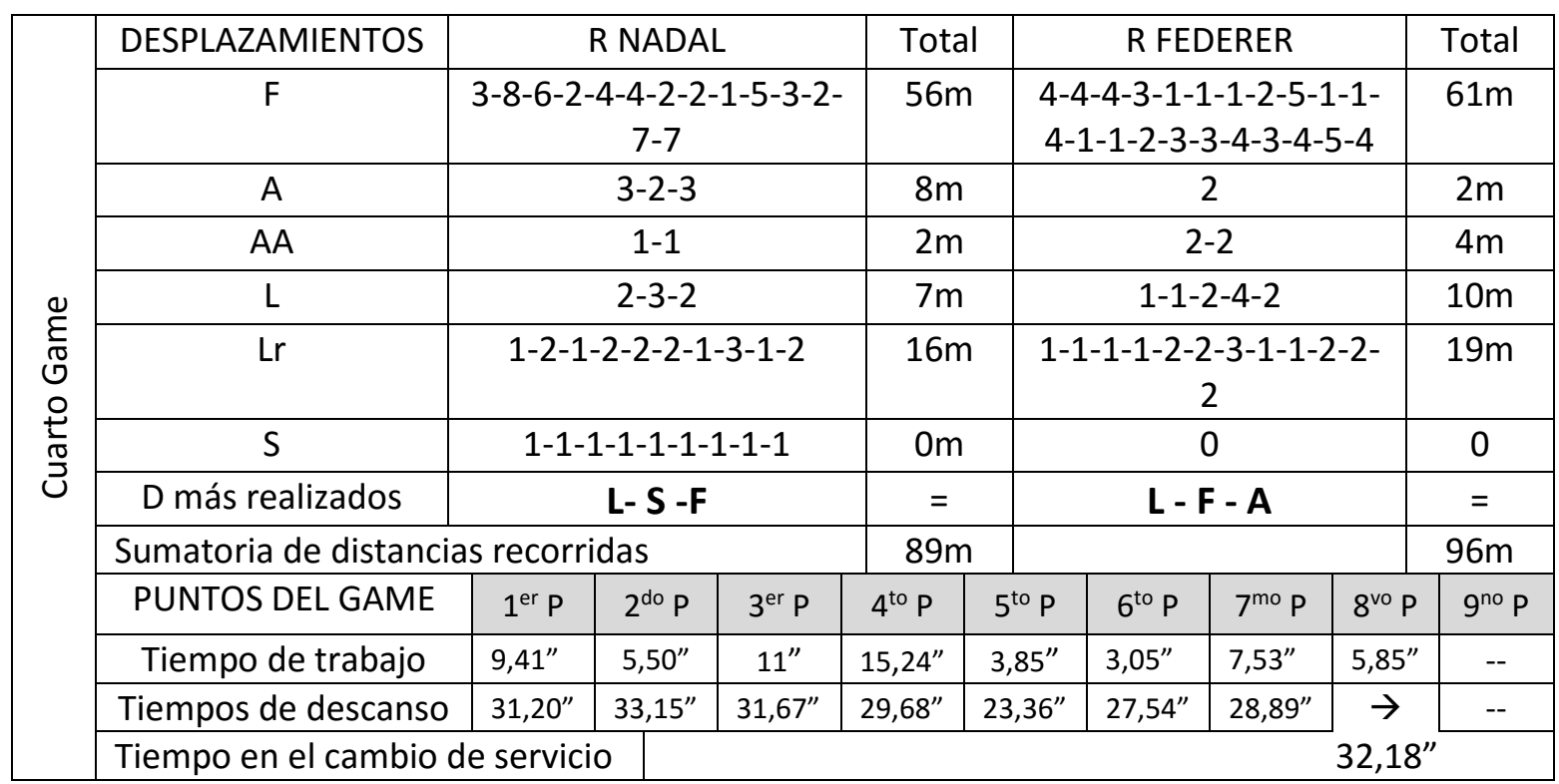

Tabla 28.

\begin{tabular}{|c|c|c|c|c|c|c|c|c|c|c|}
\hline \multirow{13}{*}{ 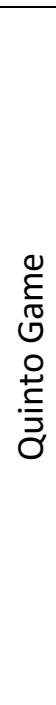 } & DESPLAZAMIENTOS & \multicolumn{3}{|c|}{ R NADAL } & \multicolumn{2}{|c|}{ Total } & \multicolumn{3}{|c|}{ R FEDERER } & Total \\
\hline & $\mathrm{F}$ & \multicolumn{3}{|c|}{$2-4-1-2-5$} & & $\mathrm{~m}$ & \multicolumn{3}{|c|}{$1-1$} & $2 m$ \\
\hline & A & \multicolumn{3}{|c|}{0} & & $\mathrm{~m}$ & \multicolumn{3}{|c|}{$2-2$} & $4 m$ \\
\hline & AA & \multicolumn{3}{|c|}{0} & & $\mathrm{~m}$ & \multicolumn{3}{|c|}{$1-1-1$} & $3 m$ \\
\hline & $\mathrm{L}$ & \multicolumn{3}{|c|}{$1-1$} & & $m$ & \multicolumn{3}{|c|}{0} & $0 m$ \\
\hline & $\mathrm{Lr}$ & \multicolumn{3}{|c|}{$2-4-2$} & & $m$ & \multicolumn{3}{|c|}{0} & $0 m$ \\
\hline & $S$ & \multicolumn{3}{|c|}{0} & & $m$ & \multicolumn{3}{|c|}{$1-1-1-1-1$} & $0 m$ \\
\hline & D más realizados & \multicolumn{3}{|c|}{ F- $L r-L$} & & $=$ & \multicolumn{3}{|c|}{ S - AA - A } & $=$ \\
\hline & \multicolumn{4}{|c|}{ Sumatoria de distancias recorridos } & \multicolumn{2}{|c|}{$24 m$} & \multicolumn{4}{|r|}{$9 m$} \\
\hline & PUNTOS DEL GAME & $1^{\text {er } P}$ & $2^{\text {do }} P$ & $3^{\text {er }} p$ & $4^{\text {to }} \mathrm{P}$ & $5^{\text {to }} \mathrm{P}$ & $6^{\text {to }} \mathrm{P}$ & $7^{\mathrm{mo}} \mathrm{P}$ & $8^{\text {vo }} \mathrm{P}$ & $9^{\text {no }} P$ \\
\hline & Tiempo de trabajo & $6,38^{\prime \prime}$ & $5,17^{\prime \prime}$ & $1,98^{\prime \prime}$ & $2,03^{\prime \prime}$ & -- & -- & -- & -- & -- \\
\hline & Tiempos de descanso & $31,20^{\prime \prime}$ & $33,15^{\prime \prime}$ & $31,67 "$ & $\rightarrow$ & -- & -- & - & -- & -- \\
\hline & \multicolumn{4}{|c|}{ Tiempo en el cambio de lado } & \multicolumn{6}{|l|}{$116^{\prime \prime}$} \\
\hline
\end{tabular}

\footnotetext{
Tabla 29.
} 


\begin{tabular}{|c|c|c|c|c|c|c|c|c|c|c|}
\hline \multirow{13}{*}{ 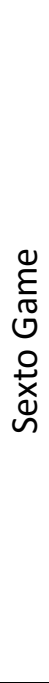 } & DESPLAZAMIENTOS & \multicolumn{3}{|c|}{ R NADAL } & \multicolumn{2}{|c|}{ Total } & \multicolumn{2}{|c|}{ R FEDERER } & \multicolumn{2}{|c|}{ Total } \\
\hline & $\mathrm{F}$ & \multicolumn{3}{|c|}{$3-1-1-1-5-7-6-1-3-1-3$} & \multicolumn{2}{|c|}{$32 m$} & \multicolumn{2}{|c|}{$2-2-3-2-4-3-1$} & \multicolumn{2}{|c|}{$17 m$} \\
\hline & A & \multicolumn{3}{|c|}{2} & \multicolumn{2}{|c|}{$2 m$} & \multicolumn{2}{|c|}{0} & \multicolumn{2}{|c|}{ Om } \\
\hline & AA & \multicolumn{3}{|c|}{0} & \multicolumn{2}{|c|}{$0 m$} & \multicolumn{2}{|c|}{0} & \multicolumn{2}{|c|}{$0 \mathrm{~m}$} \\
\hline & $\mathrm{L}$ & \multicolumn{3}{|c|}{$1-1-2$} & \multicolumn{2}{|c|}{$4 \mathrm{~m}$} & \multicolumn{2}{|c|}{1} & \multicolumn{2}{|c|}{$1 \mathrm{~m}$} \\
\hline & Lr & \multicolumn{3}{|c|}{2} & \multicolumn{2}{|c|}{$2 m$} & \multicolumn{2}{|c|}{$3-2-1-3$} & \multicolumn{2}{|c|}{$9 m$} \\
\hline & $\mathrm{S}$ & \multicolumn{3}{|c|}{ 1-1-1-1-1-1 } & \multicolumn{2}{|c|}{0} & \multicolumn{2}{|c|}{0} & \multicolumn{2}{|c|}{0} \\
\hline & D más realizados & \multicolumn{3}{|c|}{ F- S - L } & \multicolumn{2}{|c|}{$=$} & \multicolumn{2}{|c|}{$F-L r-L$} & \multicolumn{2}{|c|}{$=$} \\
\hline & \multicolumn{4}{|c|}{ Sumatoria de distancias recorridos } & \multicolumn{2}{|c|}{$40 m$} & & & \multicolumn{2}{|c|}{$27 \mathrm{~m}$} \\
\hline & PUNTOS DEL GAME & $1^{\text {er }} \mathrm{P}$ & $2^{\text {do }} p$ & $3^{\text {er }} \mathrm{P}$ & $4^{\text {to }} \mathrm{P}$ & $5^{\text {to }} \mathrm{P}$ & $6^{\text {to }} \mathrm{P}$ & $7^{\text {mo }} \mathrm{p}$ & $8^{\text {vo } P}$ & $9^{\text {no }} p$ \\
\hline & Tiempo de trabajo & $3,18^{\prime \prime}$ & $11,76^{\prime \prime}$ & $1,55^{\prime \prime}$ & $6,25^{\prime \prime}$ & -- & -- & -- & -- & -- \\
\hline & Tiempos de descanso & $23,59 "$ & $27,17^{\prime \prime}$ & $20,36 "$ & $\rightarrow$ & -- & -- & -- & -- & -- \\
\hline & Tiempo en el cambio & servic & & & 30,51 & & & & & \\
\hline
\end{tabular}

Tabla 30.

\begin{tabular}{|c|c|c|c|c|c|c|c|c|c|c|c|}
\hline \multirow{13}{*}{ 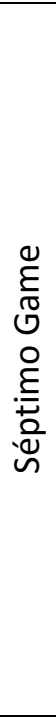 } & \multicolumn{2}{|c|}{ DESPLAZAMIENTOS } & \multicolumn{4}{|c|}{ R NADAL } & Total & \multicolumn{3}{|c|}{ R FEDERER } & Total \\
\hline & \multicolumn{2}{|c|}{$\mathrm{F}$} & \multicolumn{4}{|c|}{ 1-1-1-4-4-4-2-2-3-1-1-4-1 } & $29 m$ & \multicolumn{3}{|c|}{$5-5-3-2-3-3-3-4-1-3-9$} & $41 \mathrm{~m}$ \\
\hline & \multicolumn{2}{|l|}{ A } & \multicolumn{4}{|c|}{$3-1$} & $4 m$ & \multicolumn{3}{|c|}{$2-3-2-2-4-2$} & $15 \mathrm{~m}$ \\
\hline & \multicolumn{2}{|l|}{ AA } & \multicolumn{4}{|c|}{0} & $0 \mathrm{~m}$ & \multicolumn{3}{|c|}{ 1-1-1-1-1-1-1 } & $7 m$ \\
\hline & \multicolumn{2}{|l|}{$\mathrm{L}$} & \multicolumn{4}{|c|}{$3-2-2-2-1-1-1-2$} & $14 \mathrm{~m}$ & \multicolumn{3}{|c|}{$1-1-2-1-2-2-1$} & $10 \mathrm{~m}$ \\
\hline & \multicolumn{2}{|l|}{$\mathrm{Lr}$} & \multicolumn{4}{|c|}{$3-3-2-1-2-1-2-3-2-3$} & $22 m$ & \multicolumn{3}{|c|}{$1-2$} & $3 m$ \\
\hline & \multicolumn{2}{|l|}{$S$} & \multicolumn{4}{|c|}{0} & $0 \mathrm{~m}$ & \multicolumn{3}{|c|}{ 1-1-1-1-1-1-1-1-1-1-1 } & $0 \mathrm{~m}$ \\
\hline & \multicolumn{2}{|c|}{ D más realizados } & \multicolumn{4}{|c|}{$F-L r-L$} & $=$ & \multicolumn{3}{|c|}{ F - S - L } & $=$ \\
\hline & \multicolumn{6}{|c|}{ Sumatoria de distancias recorridos } & $69 m$ & & & & $76 m$ \\
\hline & $\begin{array}{c}\text { PUNTOS DEL } \\
\text { GAME }\end{array}$ & $1^{\text {er }} P$ & $2^{\text {do }} \mathrm{P}$ & $3^{\text {er } P}$ & $4^{\text {to }} \mathrm{P}$ & $5^{\text {to }} \mathrm{P}$ & $6^{\text {to }} \mathrm{P}$ & $7^{\text {mo }} \mathrm{P}$ & $8^{\text {vo }} \mathrm{P}$ & $9^{\text {no }} P$ & $10^{\text {mo }} \mathrm{P}$ \\
\hline & $\mathrm{T}$ trabajo & $4,17^{\prime \prime}$ & $10,35^{\prime \prime}$ & $2,16^{\prime \prime}$ & $5,75^{\prime \prime}$ & $9,83^{\prime \prime}$ & $5,19^{\prime \prime}$ & $5,02^{\prime \prime}$ & $6,08^{\prime \prime}$ & $1,61^{\prime \prime}$ & $5,26^{\prime \prime}$ \\
\hline & T descanso & $15,67^{\prime \prime}$ & $19,38^{\prime \prime}$ & $19,81^{\prime \prime}$ & $24,27 "$ & $27,21^{\prime}$ & $" 22,31^{\prime \prime}$ & $20,38^{\prime \prime}$ & $20,35^{\prime \prime}$ & $18,35^{\prime \prime}$ & $\rightarrow$ \\
\hline & Tiempo en e & ambio & de lado & & & & & & & & $116^{\prime \prime}$ \\
\hline
\end{tabular}

\section{Tabla 31.}




\begin{tabular}{|c|c|c|c|c|c|c|c|c|c|c|}
\hline \multirow{13}{*}{$\begin{array}{l} \\
\\
\stackrel{8}{E} \\
\mathbb{0} \\
0 \\
\stackrel{\overbrace{}}{0} \\
\stackrel{0}{0}\end{array}$} & DESPLAZAMIENTOS & \multicolumn{3}{|c|}{ R NADAL } & Total & \multicolumn{4}{|c|}{ R FEDERER } & Total \\
\hline & $\mathrm{F}$ & \multicolumn{3}{|c|}{$\begin{array}{c}4-2-1-3-2-5-6-2-3-2-1- \\
3-6\end{array}$} & $40 m$ & \multicolumn{4}{|c|}{$\begin{array}{c}1-1-3-5-2-3-3-2-5-2-3-3-3- \\
4-1-2-4-1-3-2-3-4-4-4-3\end{array}$} & $71 \mathrm{~m}$ \\
\hline & $A$ & \multicolumn{3}{|c|}{$2-2-2$} & $6 \mathrm{~m}$ & \multicolumn{4}{|c|}{0} & $0 m$ \\
\hline & $A A$ & \multicolumn{3}{|c|}{ 1-1-1-1-1 } & $5 \mathrm{~m}$ & \multicolumn{4}{|c|}{$1-2$} & $3 m$ \\
\hline & $\mathrm{L}$ & \multicolumn{3}{|c|}{$2-1-1-1$} & $5 \mathrm{~m}$ & \multicolumn{4}{|c|}{3} & $3 m$ \\
\hline & $\mathrm{Lr}$ & \multicolumn{3}{|c|}{$3-2-1-3-1-3-4-2-2-3$} & $24 m$ & \multicolumn{4}{|c|}{$\begin{array}{c}2-3-2-1-3-3-1-2-1-2-2-2-1- \\
1-3-3-3-2\end{array}$} & $37 \mathrm{~m}$ \\
\hline & $S$ & \multicolumn{3}{|c|}{ 1-1-1-1-1-1 } & $0 \mathrm{~m}$ & \multicolumn{4}{|c|}{0} & $0 m$ \\
\hline & D más realizados & \multicolumn{3}{|c|}{$F-\operatorname{Lr}-S$} & $=$ & \multicolumn{4}{|c|}{$F-L r-A A$} & $=$ \\
\hline & \multicolumn{4}{|c|}{ sumatoria de distancias recorridos } & $80 \mathrm{~m}$ & & & & & $114 \mathrm{~m}$ \\
\hline & PUNTOS DEL GAME & $1^{\text {er }} \mathrm{P}$ & $2^{\text {do } P}$ & $3^{\text {er }} \mathrm{P}$ & $4^{\text {to }} \mathrm{P}$ & $5^{\text {to }} \mathrm{P}$ & $6^{\text {to }} \mathrm{P}$ & $7^{\mathrm{mo}} \mathrm{p}$ & $8^{\text {vo } P}$ & $9^{\text {no }} P$ \\
\hline & Tiempos de trabajo & $1,35^{\prime \prime}$ & $6,19^{\prime \prime}$ & $12,06^{\prime \prime}$ & $6,08^{\prime \prime}$ & $12,75^{\prime \prime}$ & $28,24 "$ & -- & -- & -- \\
\hline & Tiempos de descanso & $20,87^{\prime \prime}$ & $24,78^{\prime \prime}$ & $26,48^{\prime \prime}$ & $24,83^{\prime \prime}$ & $32,03^{\prime \prime}$ & $\rightarrow$ & -- & -- & -- \\
\hline & \multicolumn{4}{|c|}{ Tiempo en el cambio de servicio } & & \multicolumn{4}{|c|}{$30,51^{\prime \prime}$} & \\
\hline
\end{tabular}

Tabla 32.

\begin{tabular}{|c|c|c|c|c|c|c|c|c|c|c|}
\hline & DESPLAZAMIENTOS & & Z NADA & & Total & & R FEL & ERER & & Total \\
\hline & $F$ & $2-3-4$ & $2-1-2-1$ & $4-3-1$ & $23 m$ & & $3-3-2-$ & $-2-1-2$ & & $15 \mathrm{~m}$ \\
\hline & A & & $2-2-1-1$ & & $6 \mathrm{~m}$ & & $3-1$ & $4-1$ & & $9 m$ \\
\hline & AA & & 0 & & $0 \mathrm{~m}$ & & 1-1- & $-1-1$ & & $5 m$ \\
\hline & $\mathrm{L}$ & & $2-2-3$ & & $7 m$ & & $1-2$ & $1-1$ & & $5 m$ \\
\hline 0 & Lr & $3-2-3-$ & $3-1-2-2-$ & $-1-3-1$ & $22 m$ & & $1-2-$ & $-2-1$ & & $8 m$ \\
\hline 요 & $S$ & & 0 & & $0 \mathrm{~m}$ & $1-1-$ & 1-1-1-1- & $-1-1-1$ & $1-1-1$ & $0 m$ \\
\hline 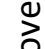 & D más realizados & & R- F - & & $=$ & & $S-F$ & - AA & & $=$ \\
\hline & Sumatoria de distanci & s recorr & das & & $42 m$ & & & & & $58 \mathrm{~m}$ \\
\hline & PUNTOS DEL GAME & $1^{\text {er }} \mathrm{P}$ & $2^{\text {do }} P$ & $3^{\text {er }} \mathrm{P}$ & $4^{\text {to }} P$ & $5^{\text {to }} \mathrm{P}$ & $6^{\text {to }} P$ & $7^{\text {mo }} \mathrm{P}$ & $8^{\text {vo } P}$ & $9^{\text {no }} P$ \\
\hline & Tiempo de trabajo & $2,09^{\prime \prime}$ & $1,65^{\prime \prime}$ & $9,79^{\prime \prime}$ & $1,75^{\prime \prime}$ & $14,04^{\prime \prime}$ & $13,35^{\prime \prime}$ & $4,65^{\prime \prime}$ & $6,11^{\prime \prime}$ & -- \\
\hline & Tiempos de descanso & $15,99^{\prime \prime}$ & $20,03^{\prime \prime}$ & $22,19^{\prime \prime}$ & $19,29^{\prime \prime}$ & $22,57 "$ & $23,54^{\prime \prime}$ & $21,68^{\prime \prime}$ & $\rightarrow$ & -- \\
\hline & Tiempo en el cambio & e lado & & & & & & & $125 "$ & \\
\hline
\end{tabular}

Tabla 33. 


\begin{tabular}{|c|c|c|c|c|c|c|c|c|c|c|}
\hline \multirow{12}{*}{ 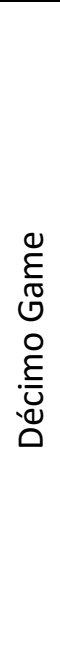 } & DESPLAZAMIENTOS & \multicolumn{3}{|c|}{ R NADAL } & Total & \multicolumn{4}{|c|}{ R FEDERER } & Total \\
\hline & $\mathrm{F}$ & \multicolumn{3}{|c|}{1} & $1 \mathrm{~m}$ & \multicolumn{4}{|c|}{$3-1-3-2-2-2-2-1-3-3$} & $22 \mathrm{~m}$ \\
\hline & A & \multicolumn{3}{|c|}{$2-1-2$} & $5 \mathrm{~m}$ & \multicolumn{4}{|c|}{0} & $0 \mathrm{~m}$ \\
\hline & AA & \multicolumn{3}{|c|}{1} & $1 \mathrm{~m}$ & \multicolumn{4}{|c|}{0} & $0 \mathrm{~m}$ \\
\hline & $L$ & \multicolumn{3}{|c|}{ 1-1-1 } & $3 m$ & \multicolumn{4}{|c|}{ 1-1-1 } & $3 m$ \\
\hline & Lr & \multicolumn{3}{|c|}{1} & $1 \mathrm{~m}$ & \multicolumn{4}{|c|}{$3-3-2$} & $8 m$ \\
\hline & $S$ & \multicolumn{3}{|c|}{ 1-1-1-1-1-1-1-1 } & $0 m$ & \multicolumn{4}{|c|}{0} & $0 m$ \\
\hline & D más realizados & \multicolumn{3}{|c|}{$S-A-L$} & $=$ & \multicolumn{4}{|c|}{ F- Lr - L } & $=$ \\
\hline & \multicolumn{4}{|c|}{ Sumatoria de distancias recorridas } & $11 \mathrm{~m}$ & & & & & $33 m$ \\
\hline & PUNTOS DEL GAME & $1^{\text {er }} P$ & $2^{\text {do }} \mathrm{P}$ & $3^{\text {er }} \mathrm{P}$ & $4^{\text {to }} \mathrm{P}$ & $5^{\text {to }} \mathrm{P}$ & $6^{\text {to }} \mathrm{P}$ & $7^{\mathrm{mo}} \mathrm{p}$ & $8^{\text {vo }} \mathrm{P}$ & $9^{\text {no }} P$ \\
\hline & Tiempo de trabajo & $1,98^{\prime \prime}$ & $1,83^{\prime \prime}$ & $7,11^{\prime \prime}$ & $2,89^{\prime \prime}$ & $7,63^{\prime \prime}$ & $1,06^{\prime \prime}$ & -- & -- & -- \\
\hline & Tiempos de descanso & $21,07^{\prime \prime}$ & $21,14^{\prime \prime}$ & $28,66 "$ & $32,98^{\prime \prime}$ & $28,11^{\prime \prime}$ & $\rightarrow$ & -- & -- & -- \\
\hline
\end{tabular}

Tabla 34.

\begin{tabular}{|c|c|c|c|c|c|c|c|c|c|c|}
\hline \multirow{12}{*}{ 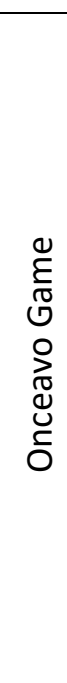 } & DESPLAZAMIENTOS & \multicolumn{3}{|c|}{ R NADAL } & \multicolumn{2}{|c|}{ Total } & \multicolumn{2}{|c|}{ R FEDERER } & \multicolumn{2}{|c|}{ Total } \\
\hline & $\mathrm{F}$ & \multicolumn{3}{|c|}{$3-1-8-3-8$} & \multicolumn{2}{|c|}{$23 m$} & \multicolumn{2}{|c|}{$6-6$} & \multicolumn{2}{|c|}{$12 m$} \\
\hline & $A$ & \multicolumn{3}{|c|}{0} & \multicolumn{2}{|c|}{$0 \mathrm{~m}$} & \multicolumn{2}{|c|}{1} & \multicolumn{2}{|c|}{$1 \mathrm{~m}$} \\
\hline & AA & \multicolumn{3}{|c|}{0} & \multicolumn{2}{|c|}{$0 \mathrm{~m}$} & \multicolumn{2}{|c|}{ 1-1-1 } & \multicolumn{2}{|c|}{$3 m$} \\
\hline & $\mathrm{L}$ & \multicolumn{3}{|c|}{$2-1$} & \multicolumn{2}{|c|}{$3 \mathrm{~m}$} & \multicolumn{2}{|c|}{ 1-1-1 } & \multicolumn{2}{|c|}{$3 m$} \\
\hline & Lr & \multicolumn{3}{|c|}{$2-1-3$} & \multicolumn{2}{|c|}{$6 m$} & \multicolumn{2}{|c|}{1} & \multicolumn{2}{|c|}{$1 \mathrm{~m}$} \\
\hline & $\mathrm{S}$ & \multicolumn{3}{|c|}{0} & \multicolumn{2}{|c|}{$0 m$} & \multicolumn{2}{|c|}{ 1-1-1-1-1-1 } & \multicolumn{2}{|c|}{$0 m$} \\
\hline & D más realizados & \multicolumn{3}{|c|}{ F- Lr - L } & \multicolumn{2}{|l|}{$=$} & \multicolumn{2}{|c|}{ S-A-L } & \multicolumn{2}{|c|}{$=$} \\
\hline & \multicolumn{4}{|c|}{ Sumatoria de distancias recorridos } & \multirow{2}{*}{\multicolumn{2}{|c|}{$32 \mathrm{~m}$}} & & & \multicolumn{2}{|c|}{$20 \mathrm{~m}$} \\
\hline & PUNTOS DEL GAME & $1^{\text {er } P}$ & $2^{\text {do } P}$ & $3^{\text {er } P}$ & & & $6^{\text {to }} P$ & $7^{\mathrm{mo}} \mathrm{P}$ & $8^{\mathrm{vo}} \mathrm{P}$ & $9^{\text {no }} P$ \\
\hline & Tiempo de trabajo & $4,06^{\prime \prime}$ & $5,88^{\prime \prime}$ & $4,32^{\prime \prime}$ & $3,23^{\prime \prime}$ & $1,81^{\prime \prime}$ & -- & -- & -- & -- \\
\hline & Tiempos de descanso & $23,58^{\prime \prime}$ & $20,89^{\prime \prime}$ & $19,60^{\prime \prime}$ & $17,49^{\prime \prime}$ & $\rightarrow$ & -- & -- & -- & -- \\
\hline
\end{tabular}

\section{Tabla 35.}




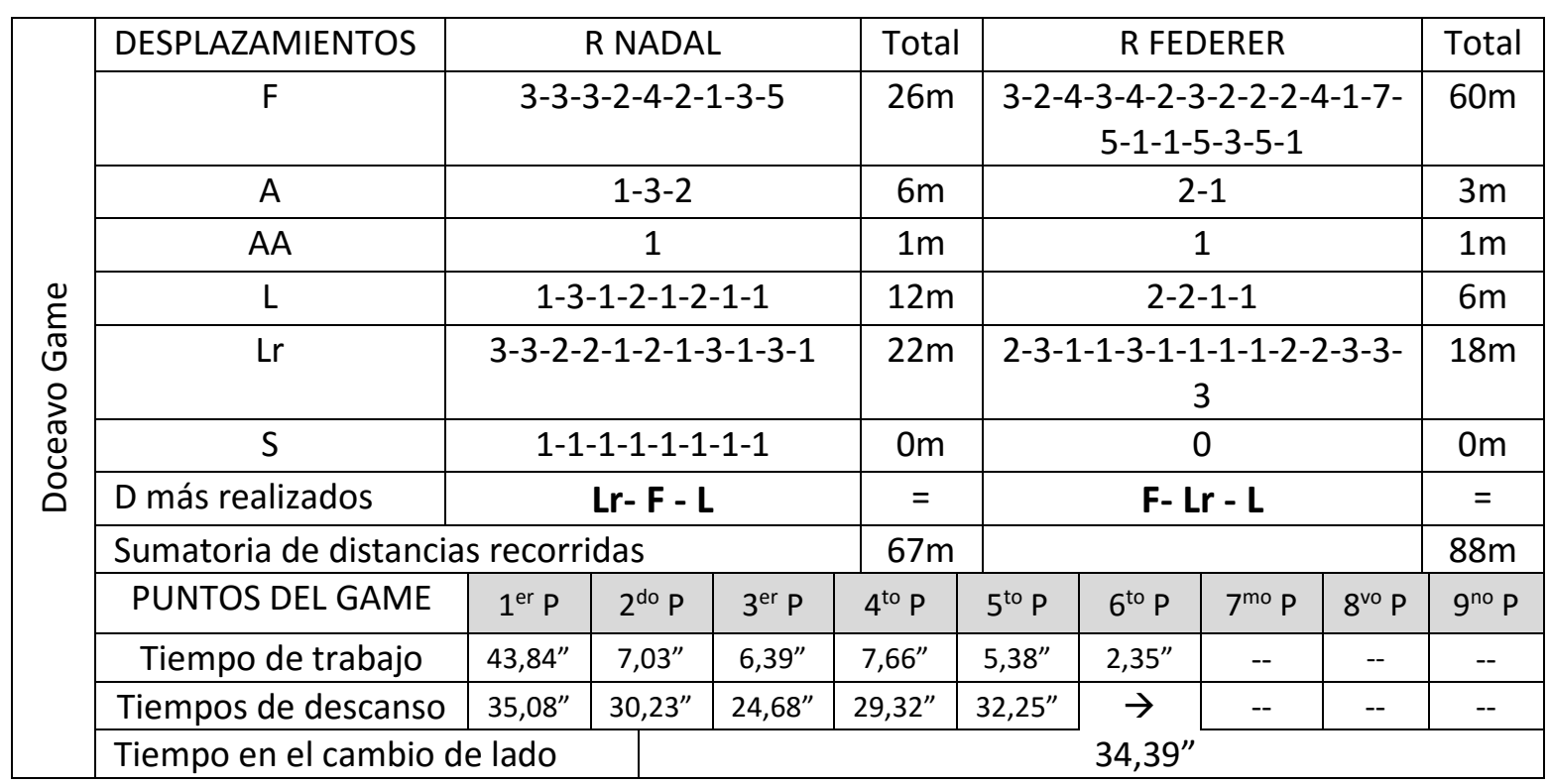

Tabla 36.

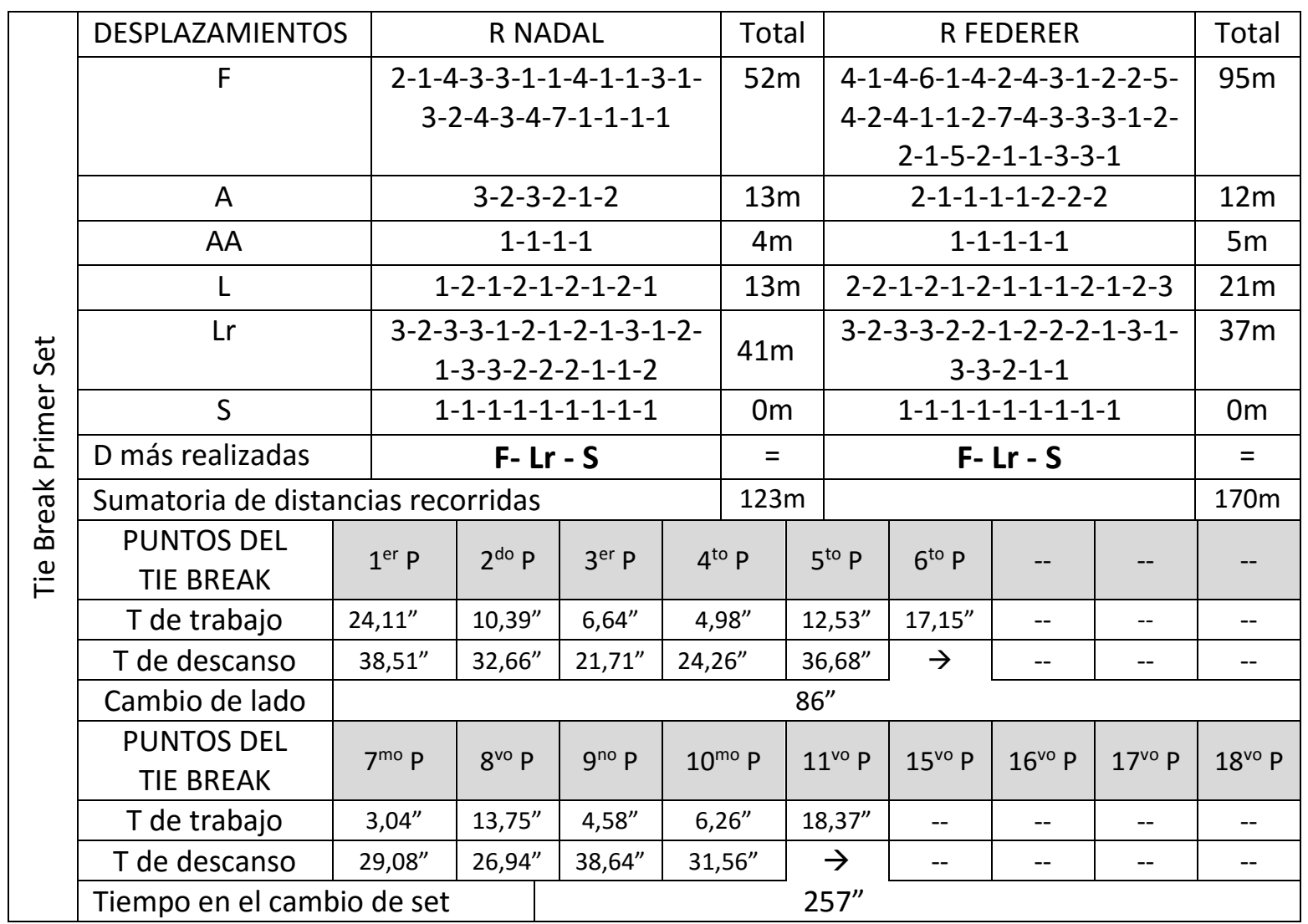

Tabla 37. 


\begin{tabular}{|c|c|c|c|c|c|c|c|}
\hline \multicolumn{8}{|c|}{$\begin{array}{l}\text { TIPOS DE DESPLAZAMIENTOS, LOS MÁS UTILIZADOS Y SUS DISTANCIAS. PRIMER SET DEL PARTIDO DE SEMIFINAL DE GRAND SLAM AUSTRALIA OPEN } \\
\text { 2014, ENTRE RAFAEL NADAL Y ROGER FEDERER. SUPERFICIE: DURA. }\end{array}$} \\
\hline $\begin{array}{c}\text { TIPOS DE } \\
\text { DESPLAZAMIENTOS }\end{array}$ & \multicolumn{4}{|c|}{ TOTAL POR SET } & $\begin{array}{l}\text { DISTANCIA MÁS } \\
\text { RECORRIDA }\end{array}$ & $\begin{array}{l}\text { SUMATORIA DE DISTANCIAS } \\
\text { RECORRIDAS POR SET }\end{array}$ & $\begin{array}{c}\text { TOTAL } \\
\text { RECORRIDO }\end{array}$ \\
\hline \multirow{7}{*}{$\begin{array}{l}\text { Desplazamientos de } \\
\quad \text { frente }\end{array}$} & $1 \mathrm{~m}$ & \multicolumn{3}{|c|}{$\begin{array}{c}* * * * * * * * * * * * * * * * * * * * * * * * * * * * * * * * * * * * * * * * * * * \\
* * * * * * * * * * * * * * * * * * * * * * * * * * * * * *\end{array}$} & & \multirow{5}{*}{$\begin{array}{l}1 \mathrm{~m}=70 \text { veces }=70 \mathrm{~m} \\
2 \mathrm{~m}=60 \text { veces }=120 \mathrm{~m} \\
3 \mathrm{~m}=56 \text { veces }=168 \mathrm{~m} \\
4 \mathrm{~m}=49 \text { veces }=196 \mathrm{~m} \\
5 \mathrm{~m}=17 \text { veces }=85 \mathrm{~m} \\
6 \mathrm{~m}=8 \text { veces }=48 \mathrm{~m} \\
7 \mathrm{~m}=7 \text { veces }=49 \mathrm{~m} \\
8 \mathrm{~m}=3 \text { veces }=24 \mathrm{~m} \\
9 \mathrm{~m}=1 \text { vez }=9 \mathrm{~m}\end{array}$} & \multirow{12}{*}{$1.439 \mathrm{~m}}$. \\
\hline & $2 m$ & $\begin{array}{r}* * * * * * * * * * * * \\
* *\end{array}$ & $\begin{array}{l}* * * * * \\
* * * * *\end{array}$ & $* * * * * * * * * * *$ & & & \\
\hline & $3 m$ & $* * * * * * * * * * * *$ & $\begin{array}{l}* * * * * \\
* * * * *\end{array}$ & $* * * * * * * * * * *$ & 1 Metro & & \\
\hline & $4 m$ & $* * * * * * * * * * * *$ & $\begin{array}{l}* * * * * \\
* * * * *\end{array}$ & $* * * * * * * * * * *$ & & & \\
\hline & $5 m$ & & $* * * *$ & & & & \\
\hline & $6 m$ & $* * * * * * * *$ & $7 \mathrm{~m}$ & $* * * * * * *$ & & \multirow{2}{*}{ 769m. } & \\
\hline & $8 m$ & $* * *$ & $9 m$ & $*$ & & & \\
\hline \multirow{3}{*}{$\begin{array}{l}\text { Desplazamientos hacia } \\
\text { atrás de ejecución }\end{array}$} & $1 \mathrm{~m}$ & \multicolumn{3}{|c|}{$* * * * * * * * * * * * * * * * * * *$} & \multirow{3}{*}{2 Metros } & \multirow{2}{*}{$\begin{array}{l}1 \mathrm{~m}=18 \text { veces }=18 \mathrm{~m} \\
2 \mathrm{~m}=28 \text { veces }=56 \mathrm{~m} \\
3 \mathrm{~m}=10 \text { veces }=30 \mathrm{~m} \\
4 \mathrm{~m}=4 \text { veces }=16 \mathrm{~m}\end{array}$} & \\
\hline & $2 m$ & $* * * * * *$ & $* * * *$ & $* * * * *$ & & & \\
\hline & $3 m$ & $* * * * * * * * * *$ & $4 m$ & $* * * * *$ & & $120 \mathrm{~m}$. & \\
\hline \multirow{2}{*}{$\begin{array}{l}\text { Desplazamientos hacia } \\
\text { atrás de recuperación }\end{array}$} & $1 \mathrm{~m}$ & $* * * * * * * * * * * *$ & $\begin{aligned} * * * * * \\
* * * *\end{aligned}$ & $* * * * * * * * * *$ & \multirow[t]{2}{*}{1 Metro } & \multirow{2}{*}{$\begin{array}{c}1 \mathrm{~m}=44 \text { veces }=44 \mathrm{~m} . \\
2 \mathrm{~m}=4 \text { veces }=8 \mathrm{~m} \\
\mathbf{5 2 m} .\end{array}$} & \\
\hline & $2 m$ & & $* * * *$ & & & & \\
\hline
\end{tabular}

Tabla 38. 


\begin{tabular}{|c|c|c|c|c|c|}
\hline \multicolumn{6}{|c|}{$\begin{array}{l}\text { TIPOS DE DESPLAZAMIENTOS, LOS MÁS UTILIZADOS Y SUS DISTANCIAS. PRIMER SET DEL PARTIDO DE SEMIFINAL DE GRAND SLAM AUSTRALIA OPEN } \\
\text { 2014, ENTRE RAFAEL NADAL Y ROGER FEDERER. SUPERFICIE: DURA. }\end{array}$} \\
\hline $\begin{array}{c}\text { TIPOS DE } \\
\text { DESPLAZAMIENTOS } \\
\end{array}$ & \multicolumn{2}{|r|}{ TOTAL POR SET } & $\begin{array}{l}\text { DISTANCIA MÁS } \\
\text { RECORRIDA }\end{array}$ & $\begin{array}{l}\text { SUMATORIA DE DISTANCIAS } \\
\text { RECORRIDAS POR SET }\end{array}$ & $\begin{array}{c}\text { TOTAL } \\
\text { RECORRIDO }\end{array}$ \\
\hline \multirow{4}{*}{$\begin{array}{l}\text { Desplazamientos } \\
\text { laterales de ejecución }\end{array}$} & $1 \mathrm{~m}$ & $\begin{array}{l}* * * * * * * * * * * * * * * * * * * * * * * * * * * * * * * * * * * * * \\
* * * * * * * * * * * * * * * * *\end{array}$ & \multirow{4}{*}{1 Metro } & \multirow{3}{*}{$\begin{array}{l}1 \mathrm{~m}=53 \text { veces }=53 \mathrm{~m} \\
2 \mathrm{~m}=36 \text { veces }=72 \mathrm{~m} \\
3 \mathrm{~m}=7 \text { veces }=21 \mathrm{~m} \\
4 \mathrm{~m}=1 \text { vez }=4 \mathrm{~m}\end{array}$} & \multirow{9}{*}{$\uparrow$} \\
\hline & $2 m$ & $* * * * * * * * * * * * * * * * * * * * * * * * * * * * * * * * * * * *$ & & & \\
\hline & $3 m$ & $* * * * * * *$ & & & \\
\hline & $4 m$ & * & & $150 \mathrm{~m}$. & \\
\hline \multirow{4}{*}{$\begin{array}{l}\text { Desplazamientos } \\
\text { laterales de } \\
\text { recuperación. }\end{array}$} & $1 \mathrm{~m}$ & $\begin{array}{l}* * * * * * * * * * * * * * * * * * * * * * * * * * * * * * * * * * * * * \\
\quad * * * * * * * * * * * * * * * * *\end{array}$ & \multirow{4}{*}{2 Metros } & \multirow{2}{*}{$\begin{array}{l}1 \mathrm{~m}=54 \text { veces }=54 \mathrm{~m} \\
2 \mathrm{~m}=68 \text { veces }=136 \mathrm{~m} \\
3 \mathrm{~m}=50 \text { veces }=150 \mathrm{~m} \\
4 \mathrm{~m}=2 \text { veces }=8 \mathrm{~m}\end{array}$} & \\
\hline & $2 m$ & $\begin{array}{c}* * * * * * * * * * * * * * * * * * * * * * * * * * * * * * * * * * * * * * \\
* * * * * * * * * * * * * * * * * * * * * * * * * * * * * * *\end{array}$ & & & \\
\hline & $3 m$ & $\begin{array}{l}* * * * * * * * * * * * * * * * * * * * * * * * * * * * * * * * * * * * * \\
* * * * * * * * * * * * * *\end{array}$ & & \multirow{2}{*}{$348 \mathrm{~m}}$. & \\
\hline & $4 m$ & $* *$ & & & \\
\hline $\begin{array}{l}\text { Desplazamientos hacia } \\
\text { arriba o saltos }\end{array}$ & \multicolumn{2}{|c|}{$\begin{array}{c}* * * * * * * * * * * * * * * * * * * * * * * * * * * * * * * * * * * * * * * * * * * * \\
* * * * * * * * * * * * * * * * * * * * * * * * * * * * * * * * * * * * * * * * * * * \\
* * * * * * * * * * * *\end{array}$} & \multicolumn{2}{|c|}{ Saltos en servicio $=108$ veces } & \\
\hline
\end{tabular}

Tabla39.

\begin{tabular}{|c|c|c|c|c|c|c|}
\hline \multicolumn{7}{|c|}{$\begin{array}{l}\text { PORCENTAJES DE USO DE LOS DIFERENTES TIPOS DE DESPLAZAMIENTOS EN EL PRIMER SET DEL PARTIDO DE SEMIFINAL DE GRAND SLAM AUSTRALIA } \\
\text { OPEN 2014, ENTRE RAFAEL NADAL Y ROGER FEDERER. SUPERFICIE: DURA. }\end{array}$} \\
\hline & D HACIA ADELANTE & $\begin{array}{l}\text { D HACIA ATRÁS } \\
\text { DE EJECUCIÓN }\end{array}$ & $\begin{array}{l}\text { D HACIA ATRÁS DE } \\
\text { RECUPERACIÓN }\end{array}$ & $\begin{array}{l}\text { D LATERALES DE } \\
\text { EJECUCIÓN }\end{array}$ & $\begin{array}{l}\text { D LATERALES DE } \\
\text { RECUPERACIÓN }\end{array}$ & D HACIA ARRIBA \\
\hline NÚMERO DE VECES & $35,75 \%$ & $7,91 \%$ & $6,33 \%$ & $12,79 \%$ & $22,95 \%$ & $14,24 \%$ \\
\hline DISTANCIA & $53,43 \%$ & $8,33 \%$ & $3,61 \%$ & $10,42 \%$ & $24,18 \%$ & \\
\hline
\end{tabular}

Tabla40. 


\begin{tabular}{|c|c|c|c|c|}
\hline \multicolumn{5}{|c|}{$\begin{array}{l}\text { TIEMPOS GLOBALES DE LOS MOMENTOS DE TRABAJO Y PAUSA DEL PRIMER SET DEL PARTIDO ENTRE } \\
\text { ROGER FEDERER y RAFAEL NADAL, EN SEMIFINALES DEL AUSTRALIAN OPEN } 2014\end{array}$} \\
\hline TIEMPOS & \multicolumn{2}{|c|}{ SUMATORIA DE } & $\begin{array}{c}\text { SUMATORIA DE TIEMPOS } \\
\text { POR SET }\end{array}$ & $\begin{array}{l}\text { RELACIÓN TIEMPO } \\
\text { DE TRABAJO PAUSA }\end{array}$ \\
\hline \multirow{13}{*}{ TIEMPO DE TRABAJO } & $1^{\text {er }}$ Game & $18,59^{\prime \prime}$ & \multirow{13}{*}{$\begin{array}{l}600,1 " \text { tiempo real de } \\
\text { trabajo }\end{array}$} & \multirow{26}{*}{$\begin{array}{c}\text { 1" de Trabajo. } \\
x \\
\text { 4,63" de Pausa. }\end{array}$} \\
\hline & $2^{\text {do }}$ Game & $19,71^{\prime \prime}$ & & \\
\hline & $3^{\text {er }}$ Game & $50,57^{\prime \prime}$ & & \\
\hline & $4^{\text {to }}$ Game & $61,43^{\prime \prime}$ & & \\
\hline & $5^{\text {to }}$ Game & $15,56^{\prime \prime}$ & & \\
\hline & $6^{\text {to }}$ Game & $22,74^{\prime \prime}$ & & \\
\hline & $7^{\text {to }}$ Game & $55,42 "$ & & \\
\hline & $8^{\text {vo }}$ Game & $66,67 "$ & & \\
\hline & $9^{\text {no }}$ Game & $53,43^{\prime \prime}$ & & \\
\hline & $10^{\mathrm{mo}}$ Game & $22,5^{\prime \prime}$ & & \\
\hline & $11^{\text {vo }}$ Game & $19,03^{\prime \prime}$ & & \\
\hline & $12^{\mathrm{vo}}$ Game & $72,65^{\prime \prime}$ & & \\
\hline & Tie Break & $121,8^{\prime \prime}$ & & \\
\hline \multirow{13}{*}{ TIEMPO DE DESCANSO } & $1^{\mathrm{er}}$ Game & $152,72^{\prime \prime}$ & \multirow{13}{*}{$\begin{array}{c}2.782,84 " \text { Tiempo real de } \\
\text { descanso }\end{array}$} & \\
\hline & $2^{\text {do }}$ Game & $96,56 "$ & & \\
\hline & $3^{\text {er }}$ Game & $217,57^{\prime \prime}$ & & \\
\hline & $4^{\text {to }}$ Game & $237,67^{\prime \prime}$ & & \\
\hline & $5^{\text {to }}$ Game & $212,02^{\prime \prime}$ & & \\
\hline & $6^{\text {to }}$ Game & $101,63^{\prime \prime}$ & & \\
\hline & $7^{\text {to }}$ Game & $303,73^{\prime \prime}$ & & \\
\hline & $8^{\text {vo }}$ Game & $159,5^{\prime \prime}$ & & \\
\hline & $9^{\text {no }}$ Game & $270,29 "$ & & \\
\hline & $10^{\text {mo }}$ Game & $165,6^{\prime \prime}$ & & \\
\hline & $11^{\text {vo }}$ Game & $194,56^{\prime \prime}$ & & \\
\hline & $12^{\mathrm{vo}}$ Game & $185,95^{\prime \prime}$ & & \\
\hline & Tie Break & $485,04 "$ & & \\
\hline
\end{tabular}

Tabla41 


\begin{tabular}{|c|c|c|c|}
\hline \multicolumn{4}{|c|}{$\begin{array}{l}\text { PROMEDIO ESPECÍFICOS DE LOS MOMENTOS DE TRABAJO Y PAUSA DEL PRIMER SET DEL PARTIDO } \\
\text { ENTRE ROGER FEDERER y RAFAEL NADAL, EN SEMIFINALES DEL AUSTRALIAN OPEN } 2014\end{array}$} \\
\hline \multicolumn{2}{|c|}{ PROMEDIOS DE TRABAJO } & \multicolumn{2}{|c|}{ PROMEDIOS DE PAUSA } \\
\hline $1^{\text {er }}$ Game & $3,71^{\prime \prime}$ & $1^{\text {er }}$ Game & 16,93" \\
\hline $2^{\text {do }}$ Game & 4,92" & $2^{\text {do }}$ Game & $22,25^{\prime \prime}$ \\
\hline $3^{\text {er }}$ Game & $8,42^{\prime \prime}$ & $3^{\text {er }}$ Game & $18,4^{\prime \prime}$ \\
\hline $4^{\text {to }}$ Game & 7,67" & $4^{\text {to }}$ Game & $29,35^{\prime \prime}$ \\
\hline $5^{\text {to }}$ Game & $3,89^{\prime \prime}$ & $5^{\text {to }}$ Game & $32 "$ \\
\hline $6^{\text {to }}$ Game & $5,68^{\prime \prime}$ & $6^{\text {to }}$ Game & $23,70^{\prime \prime}$ \\
\hline $7^{\mathrm{mo}}$ Game & $5,54^{\prime \prime}$ & $7^{\mathrm{mo}}$ Game & $20,85^{\prime \prime}$ \\
\hline $8^{\text {vo }}$ Game & $11,11^{\prime \prime}$ & $8^{\text {vo }}$ Game & $25,79^{\prime \prime}$ \\
\hline $9^{\text {no }}$ Game & 6,67" & $9^{\text {no }}$ Game & $20,75^{\prime \prime}$ \\
\hline $10^{\mathrm{mo}}$ Game & $3,75^{\prime \prime}$ & $10^{\mathrm{mo}}$ Game & $26,39^{\prime \prime}$ \\
\hline $11^{\text {vo }}$ Game & $3,80^{\prime \prime}$ & $11^{\text {vo }}$ Game & $20,39^{\prime \prime}$ \\
\hline $12^{\text {vo }}$ Game & $12,10^{\prime \prime}$ & $12^{\text {vo }}$ Game & $30,31^{\prime \prime}$ \\
\hline Tie Break & $11,07^{\prime \prime}$ & Tie Break & $31,11^{\prime \prime}$ \\
\hline \multicolumn{2}{|c|}{ Promedio total } & \multicolumn{2}{|c|}{ Promedio total } \\
\hline \multicolumn{2}{|c|}{$6,79^{\prime \prime}$} & \multicolumn{2}{|c|}{$24,47^{\prime \prime}$} \\
\hline \multicolumn{4}{|c|}{ 1" de trabajo por 3,60 " de pausa } \\
\hline
\end{tabular}

Tabla42.

3. Análisis punto a punto del primer set, del partido entre Paula Ormaechea (Arg) y Teliana Pereira (Bra), en la copa claro Colsánitas Bogotá 2013. En la que, la Argentina $P$ Ormaechea le ganó a la Brasileña T Pereira 7-6 ${ }^{(7-5)}$ y 6-3. Tipo de cancha (polvo de ladrillo). clasificación ITF categoría 1 (velocidad baja). Ver tablas de 43 a 60.

El análisis del peloteo previo al comienzo del partido, no se pudo hacer, pues no lo permitió el vídeo del torneo, así que lo obviamos y pasamos directamente a consignar en las siguientes tablas, los resultados de los tipos de desplazamientos, distancias recorridas y tiempos de trabajo pausa del primer set del partido. 


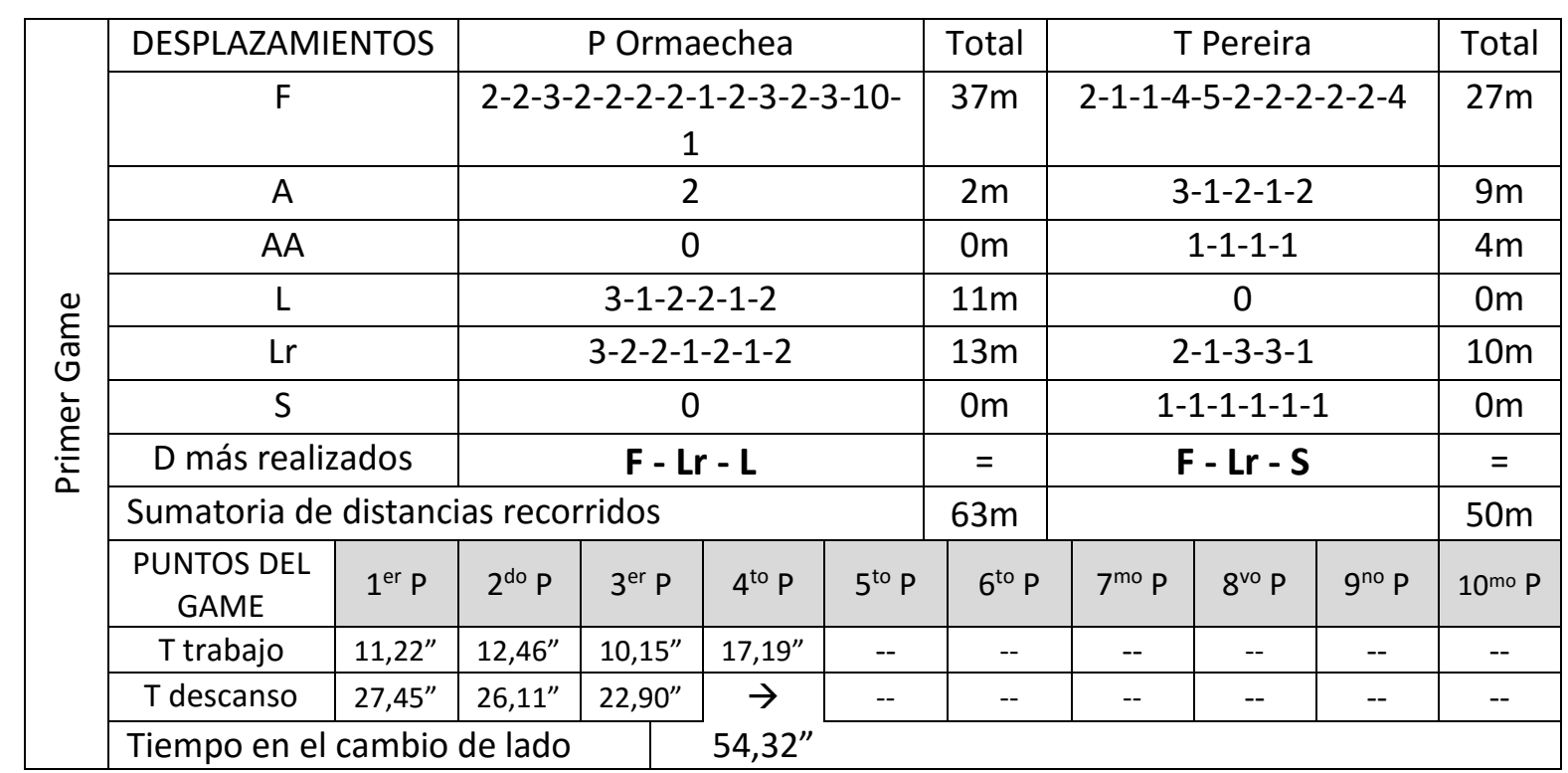

Tabla 43.

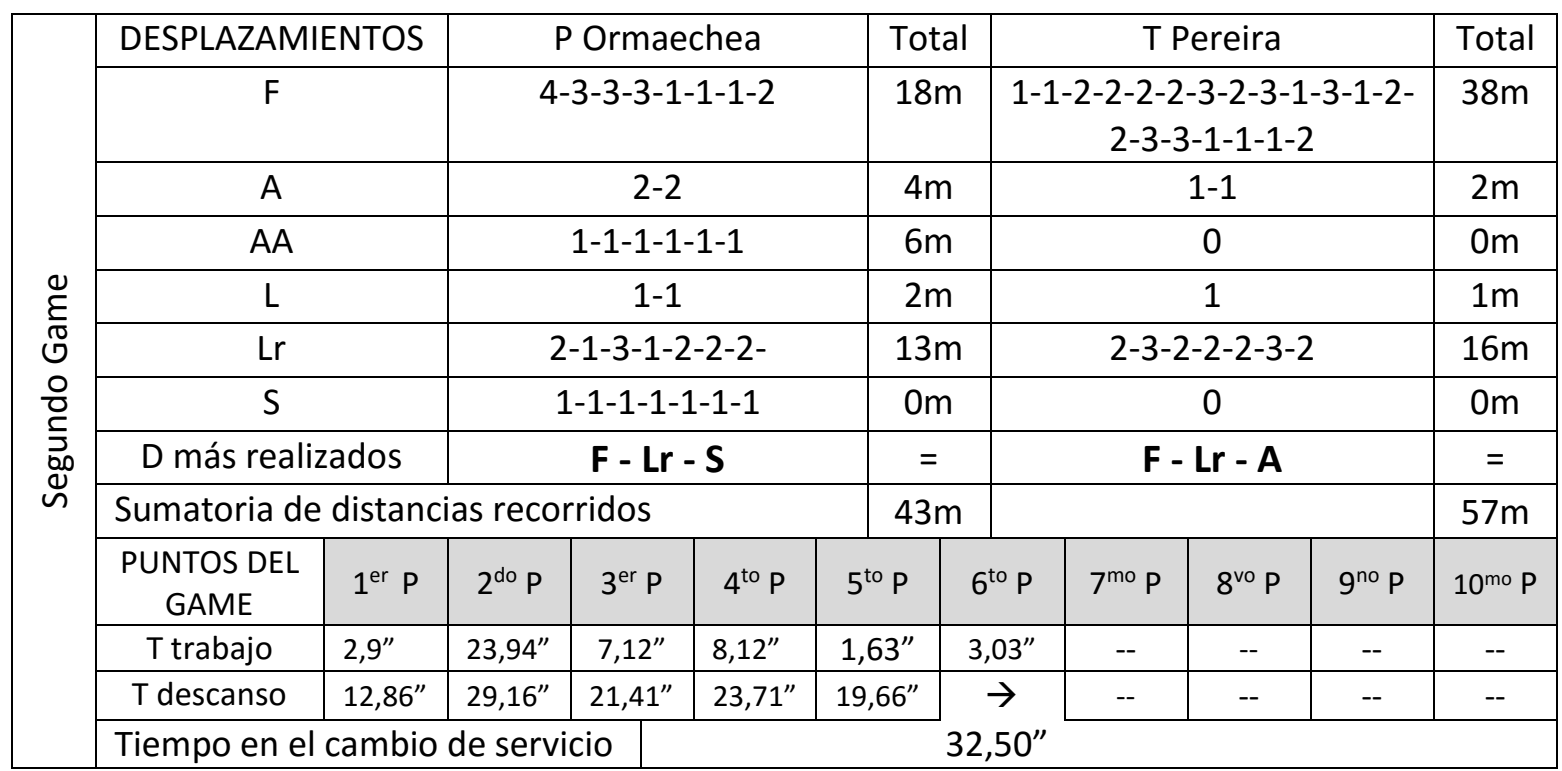

Tabla 44. 


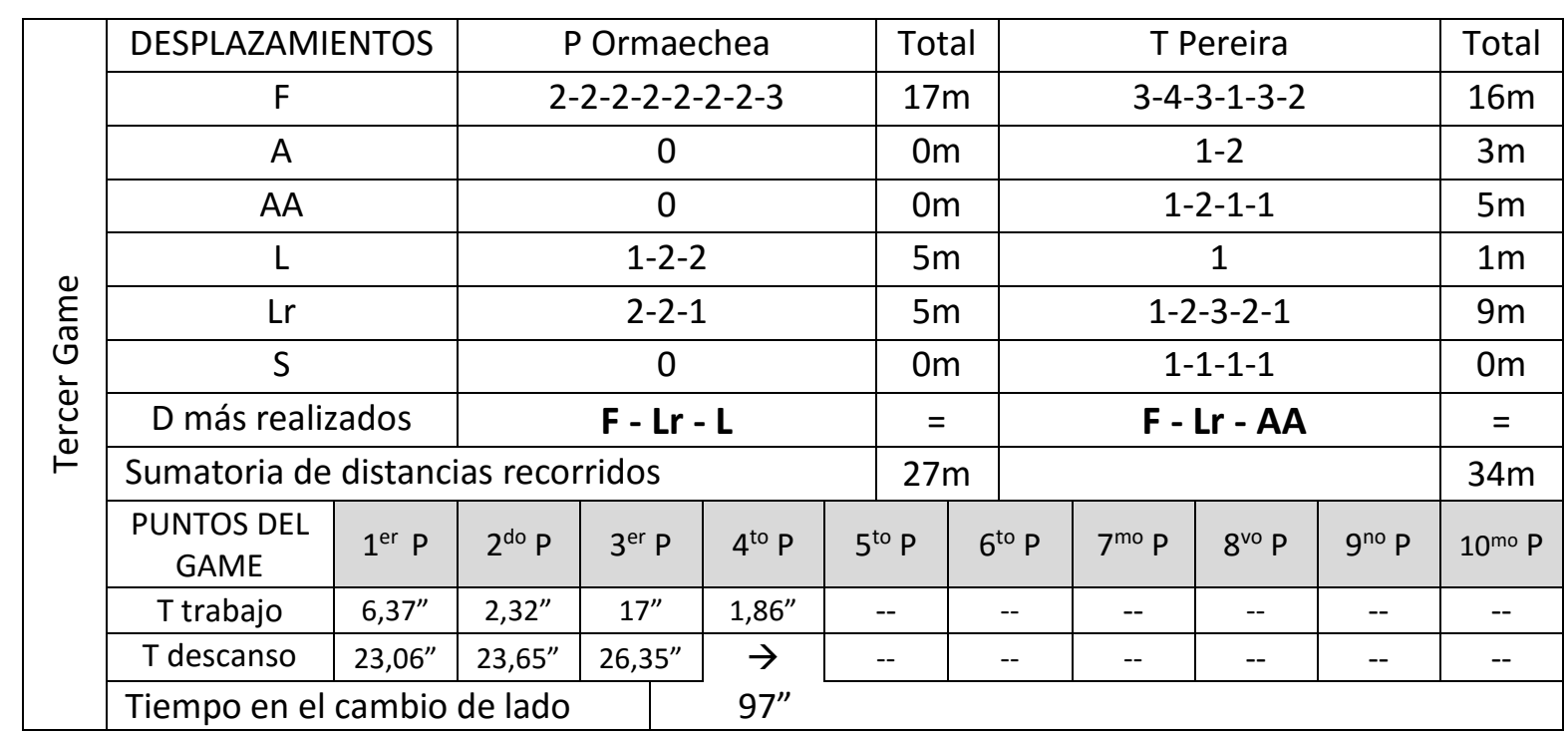

Tabla 45.

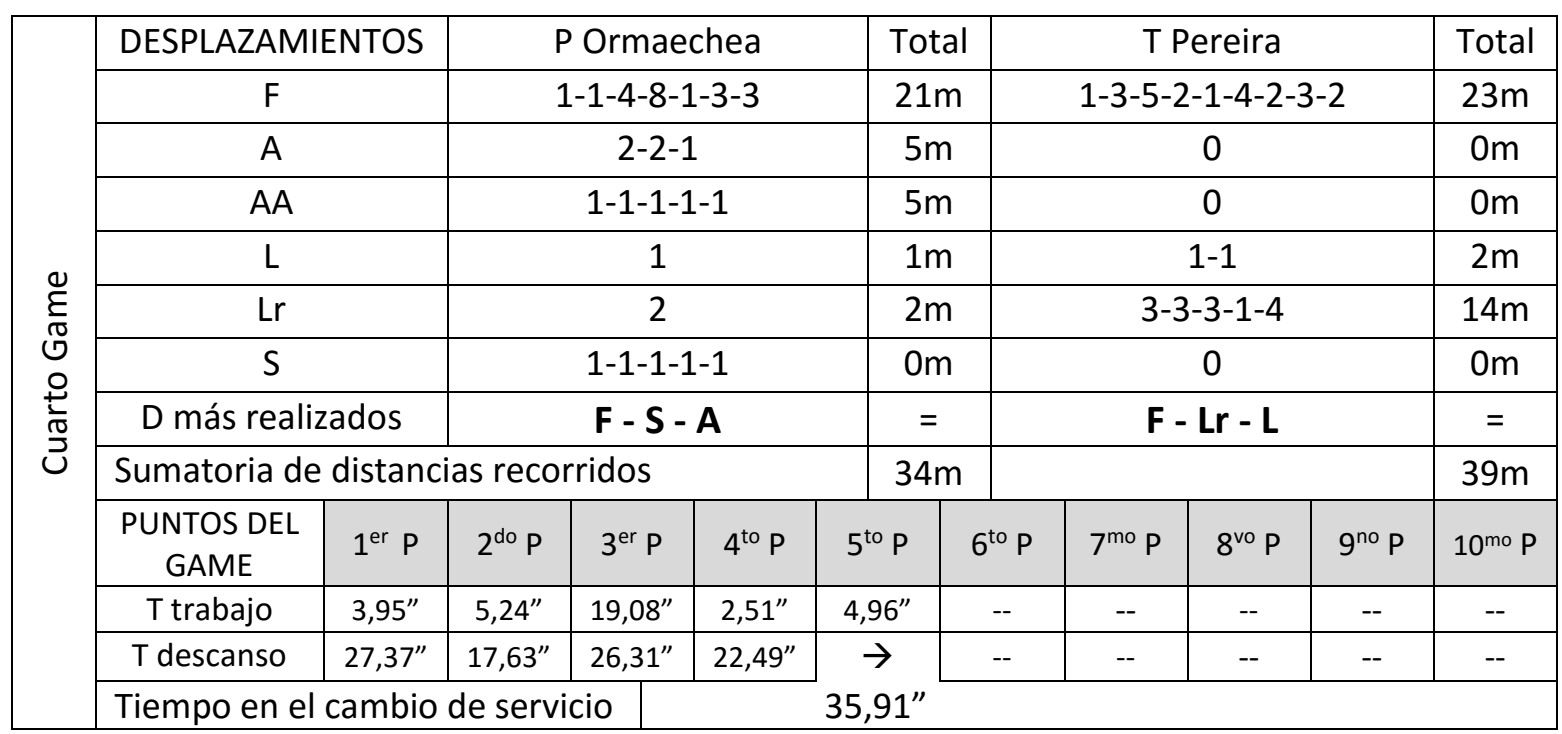

\section{Tabla 46.}




\begin{tabular}{|c|c|c|c|c|c|c|c|c|c|c|c|c|}
\hline \multirow{13}{*}{ 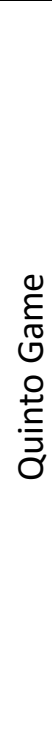 } & \multicolumn{2}{|c|}{ DESPLAZAMIENTOS } & \multicolumn{4}{|c|}{ P Ormaechea } & Total & \multicolumn{4}{|c|}{ T Pereira } & Total \\
\hline & \multicolumn{2}{|l|}{$\mathrm{F}$} & \multicolumn{4}{|c|}{$\begin{array}{c}1-1-2-3-2-1-2-1-3-1-2-4- \\
2-2-2-1-1-1-\end{array}$} & $34 \mathrm{~m}$ & \multicolumn{4}{|c|}{$1-2-1-2-2-2-2-1$} & $13 \mathrm{~m}$ \\
\hline & \multicolumn{2}{|l|}{ A } & \multicolumn{3}{|c|}{ 1-1-1-1- } & \multicolumn{2}{|r|}{$4 m$} & \multicolumn{4}{|c|}{ 1-1-2-2-1- } & $7 \mathrm{~m}$ \\
\hline & \multicolumn{2}{|l|}{ AA } & \multicolumn{3}{|c|}{1} & \multicolumn{2}{|r|}{$1 \mathrm{~m}$} & \multicolumn{4}{|c|}{ 1-1-1-1-1-1-1-1-1-1-1 } & $11 \mathrm{~m}$ \\
\hline & \multicolumn{2}{|l|}{$\mathrm{L}$} & \multicolumn{3}{|c|}{$1-2-1$} & \multicolumn{2}{|r|}{$4 m$} & \multicolumn{4}{|c|}{$1-1-2-1$} & $5 m$ \\
\hline & \multicolumn{2}{|l|}{ Lr } & \multicolumn{3}{|c|}{$3-3-2-3-3-3-2-1-3-2-1$} & \multicolumn{2}{|c|}{$26 \mathrm{~m}$} & \multicolumn{4}{|c|}{$1-1-2$} & $4 m$ \\
\hline & \multicolumn{2}{|l|}{$S$} & \multicolumn{3}{|c|}{0} & \multicolumn{2}{|r|}{$0 \mathrm{~m}$} & \multicolumn{4}{|c|}{ 1-1-1-1-1-1-1-1-1-1-1-1-1 } & Om \\
\hline & \multicolumn{2}{|c|}{ D más realizados } & \multicolumn{3}{|c|}{$F-L r-A$} & \multicolumn{2}{|r|}{$=$} & \multicolumn{4}{|c|}{$F-S-A A$} & $=$ \\
\hline & \multicolumn{5}{|c|}{ Sumatoria de distancias recorridos } & \multicolumn{2}{|c|}{$69 \mathrm{~m}$} & & $40 \mathrm{~m}$ \\
\hline & $\begin{array}{l}\text { PUNTOS DEL } \\
\text { GAME }\end{array}$ & $1^{\text {er }} \mathrm{P}$ & $2^{\text {do }} \mathrm{P}$ & $3^{\text {er } P}$ & $4^{\text {to }} \mathrm{P}$ & $5^{\text {to }} \mathrm{P}$ & & $6^{\text {to }} \mathrm{P}$ & $7^{\text {mo }} p$ & $8^{\mathrm{vo}} \mathrm{P}$ & $9^{\text {no }} \mathrm{P}$ & $10^{\text {mo }} \mathrm{P}$ \\
\hline & $\mathrm{T}$ trabajo & $8,36^{\prime \prime}$ & $2,18^{\prime \prime}$ & $6,79^{\prime \prime}$ & $5,34^{\prime \prime}$ & $6,28^{\prime}$ & & $5,32^{\prime \prime}$ & $6,51^{\prime \prime}$ & $5,49^{\prime \prime}$ & -- & -- \\
\hline & T descanso & $24,73^{\prime \prime}$ & $23,07 "$ & $22,32^{\prime \prime}$ & $26,66^{\prime \prime}$ & 23,51 & & $9,11^{\prime \prime}$ & $28,95^{\prime \prime}$ & $\rightarrow$ & -- & -- \\
\hline & Tiempo en e & cambio & de lado & & & & & & & 97" & & \\
\hline
\end{tabular}

\section{Tabla 47.}

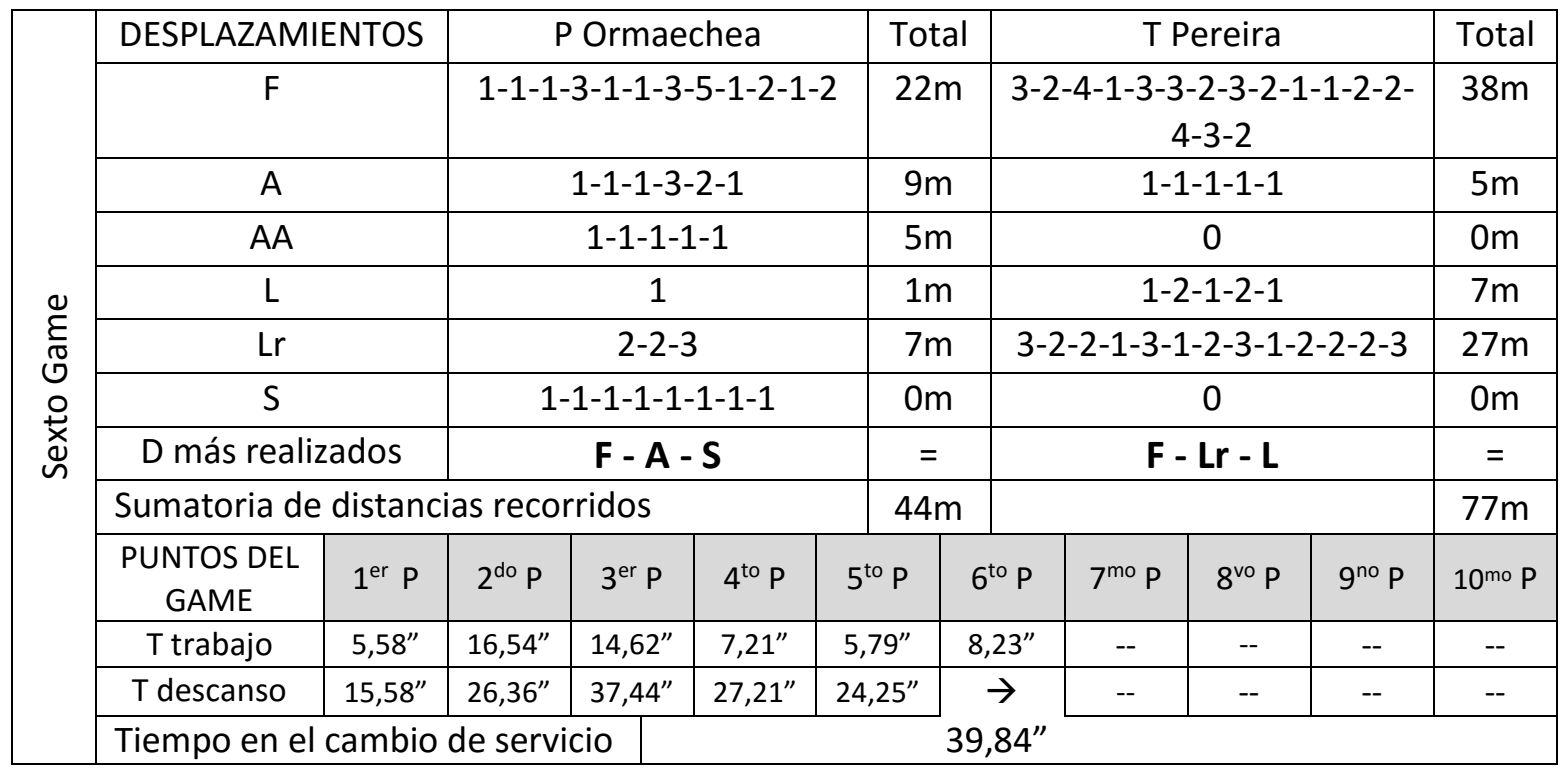

Tabla 48. 


\begin{tabular}{|c|c|c|c|c|c|c|c|c|c|c|c|}
\hline \multirow{13}{*}{ 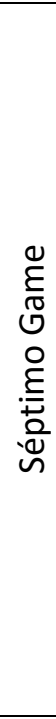 } & \multirow{2}{*}{\multicolumn{2}{|c|}{ DESPLAZAMIENTOS }} & \multicolumn{3}{|c|}{ P Ormaechea } & \multicolumn{2}{|c|}{ Total } & \multicolumn{3}{|c|}{ T Pereira } & Total \\
\hline & & & \multicolumn{3}{|c|}{ 1-2-2-1-2-3-1-4-3-1-1-2 } & \multicolumn{2}{|c|}{$23 m$} & \multicolumn{3}{|c|}{$2-1-1-1-4-1-2-1-3-1$} & $17 m$ \\
\hline & \multicolumn{2}{|l|}{ A } & \multicolumn{3}{|c|}{0} & \multicolumn{2}{|c|}{$0 m$} & \multicolumn{3}{|c|}{1} & $1 \mathrm{~m}$ \\
\hline & \multicolumn{2}{|l|}{ AA } & \multicolumn{3}{|c|}{0} & \multicolumn{2}{|c|}{$0 \mathrm{~m}$} & \multicolumn{3}{|c|}{$2-1-1-2$} & $6 m$ \\
\hline & \multicolumn{2}{|l|}{$\mathrm{L}$} & \multicolumn{3}{|c|}{2} & \multicolumn{2}{|c|}{$2 \mathrm{~m}$} & \multicolumn{3}{|c|}{2} & $2 m$ \\
\hline & \multicolumn{2}{|l|}{ Lr } & \multicolumn{3}{|c|}{$1-1-1-3-2-2$} & \multicolumn{2}{|c|}{$10 \mathrm{~m}$} & \multicolumn{3}{|c|}{$2-2-3$} & $7 m$ \\
\hline & \multicolumn{2}{|l|}{$S$} & \multicolumn{3}{|c|}{0} & \multicolumn{2}{|c|}{$0 \mathrm{~m}$} & \multicolumn{3}{|c|}{ 1-1-1-1-1-1 } & $0 m$ \\
\hline & \multicolumn{2}{|c|}{ D más realizados } & \multicolumn{3}{|c|}{$F-L r-L$} & & & \multicolumn{3}{|c|}{$F-\operatorname{Lr}-\mathbf{A A}$} & $=$ \\
\hline & \multicolumn{5}{|c|}{ Sumatoria de distancias recorridos } & \multicolumn{2}{|c|}{$35 \mathrm{~m}$} & & & & $33 m$ \\
\hline & $\begin{array}{c}\text { PUNTOS DEL } \\
\text { GAME }\end{array}$ & $1^{\text {er }} P$ & $2^{\text {do }} \mathrm{P}$ & $3^{\text {er }} \mathrm{P}$ & $4^{\text {to }} \mathrm{P}$ & $5^{\text {to }} \mathrm{P}$ & $6^{\text {to } P}$ & $7^{\mathrm{mo}} \mathrm{P}$ & $8^{\mathrm{vo}} \mathrm{P}$ & $9^{\text {no }} P$ & $10^{\text {mo }} \mathrm{P}$ \\
\hline & T trabajo & $14,77^{\prime \prime}$ & $2,19^{\prime \prime}$ & $7,79^{\prime \prime}$ & $5,42^{\prime \prime}$ & -- & -- & -- & -- & -- & -- \\
\hline & T descanso & $28^{\prime \prime}$ & $20,60^{\prime \prime}$ & $21,37^{\prime \prime}$ & $\rightarrow$ & -- & -- & -- & -- & -- & -- \\
\hline & Tiempo en el & cambio & e serv & & $92^{\prime \prime}$ & & & & & & \\
\hline
\end{tabular}

\section{Tabla 49.}

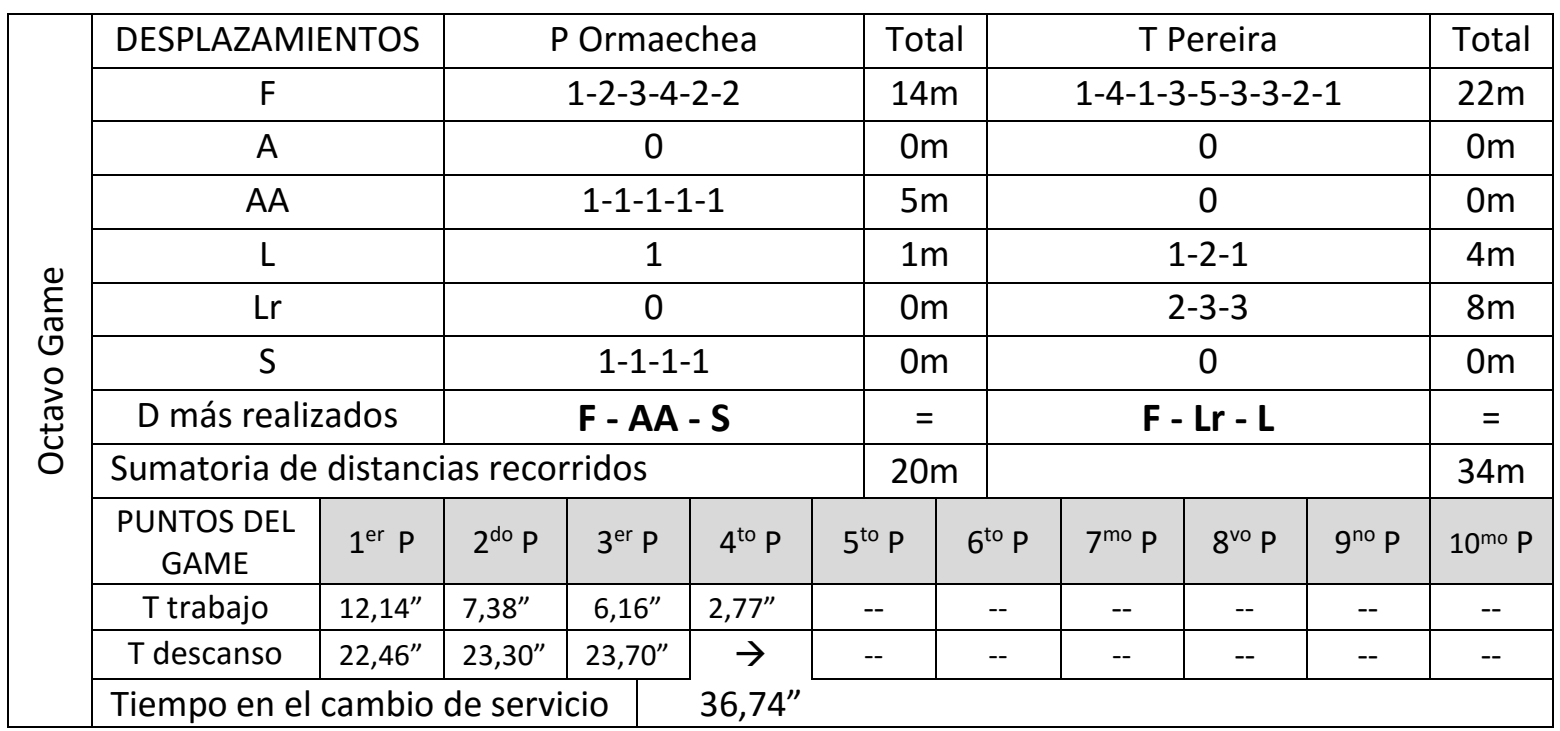

\section{Tabla 50.}




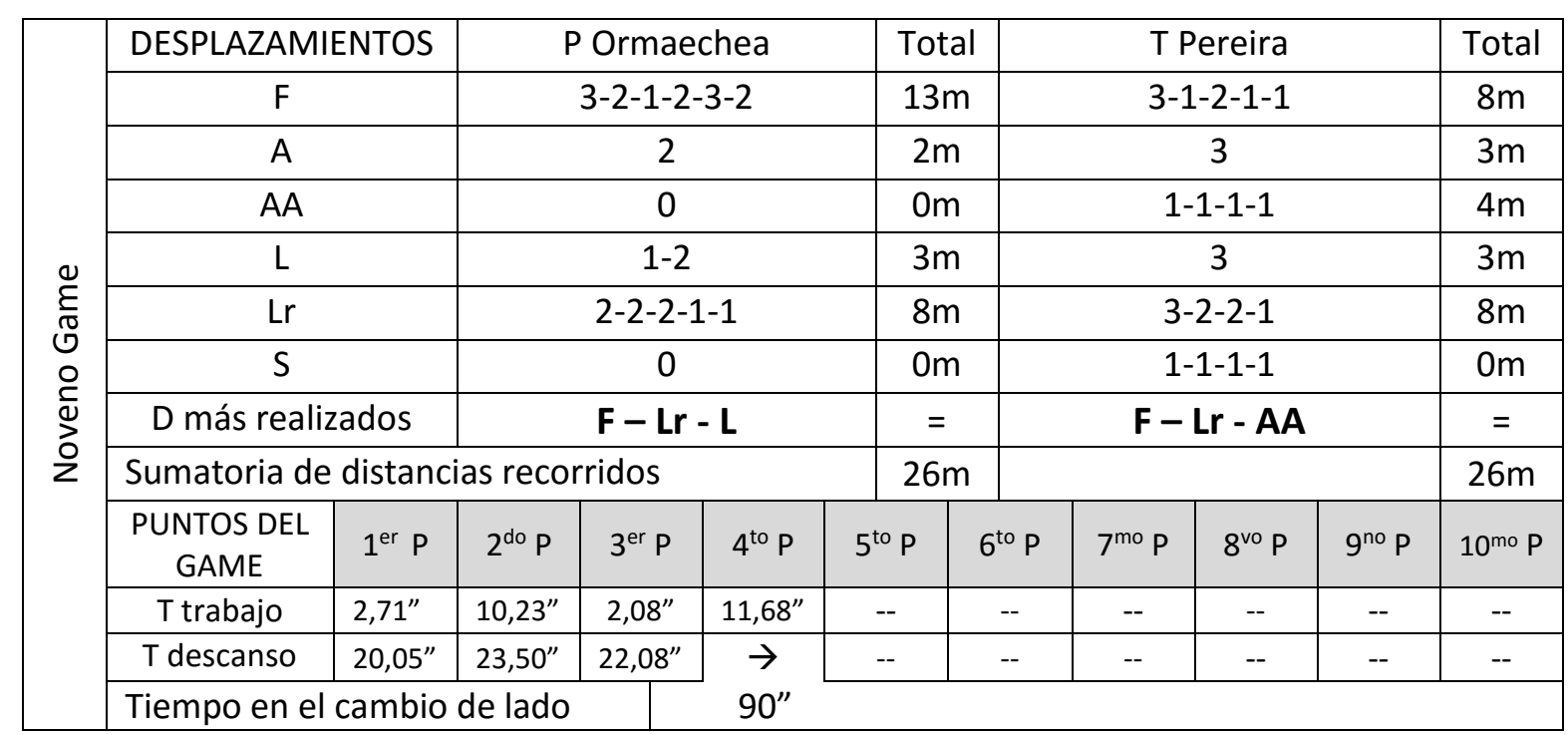

Tabla 51.

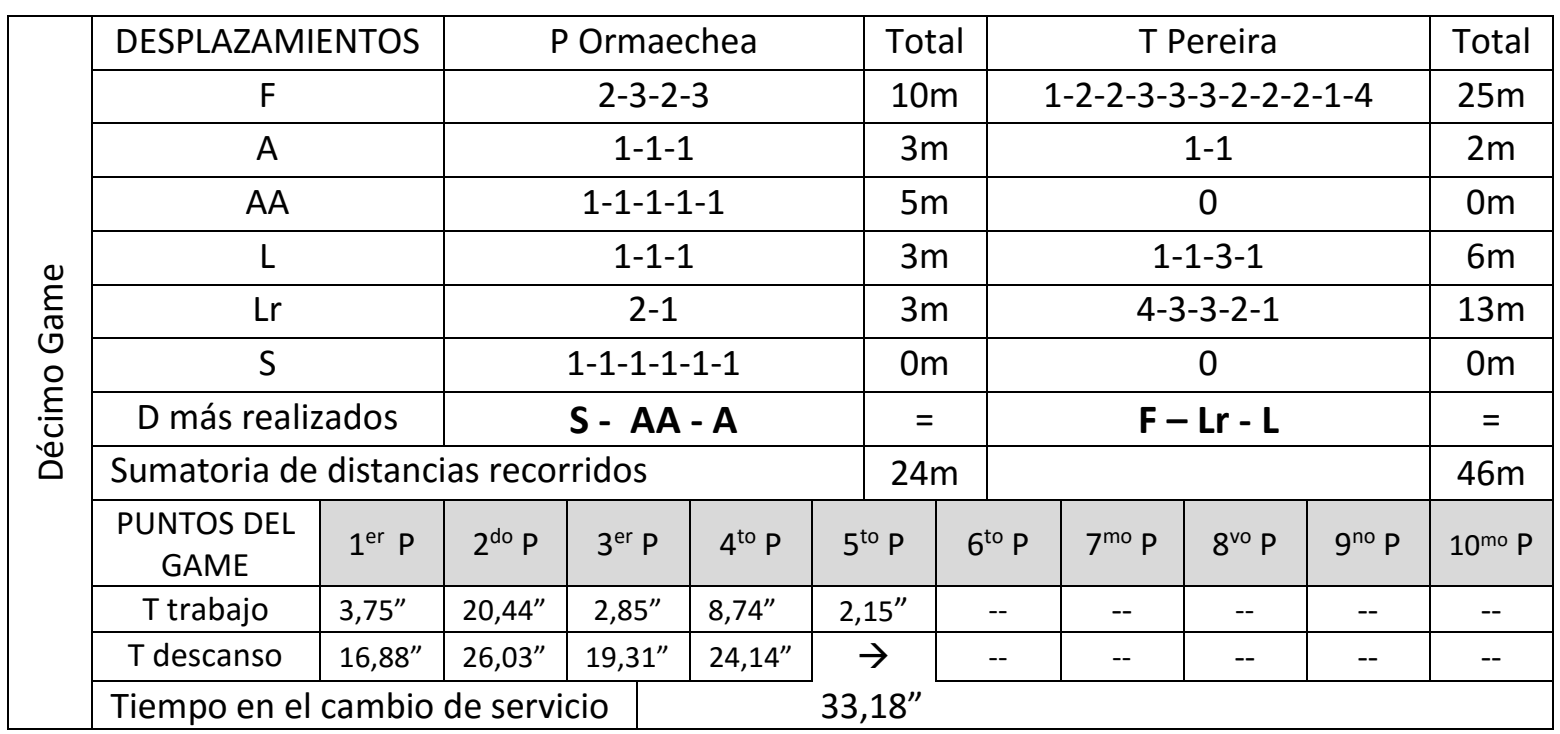

Tabla 52. 


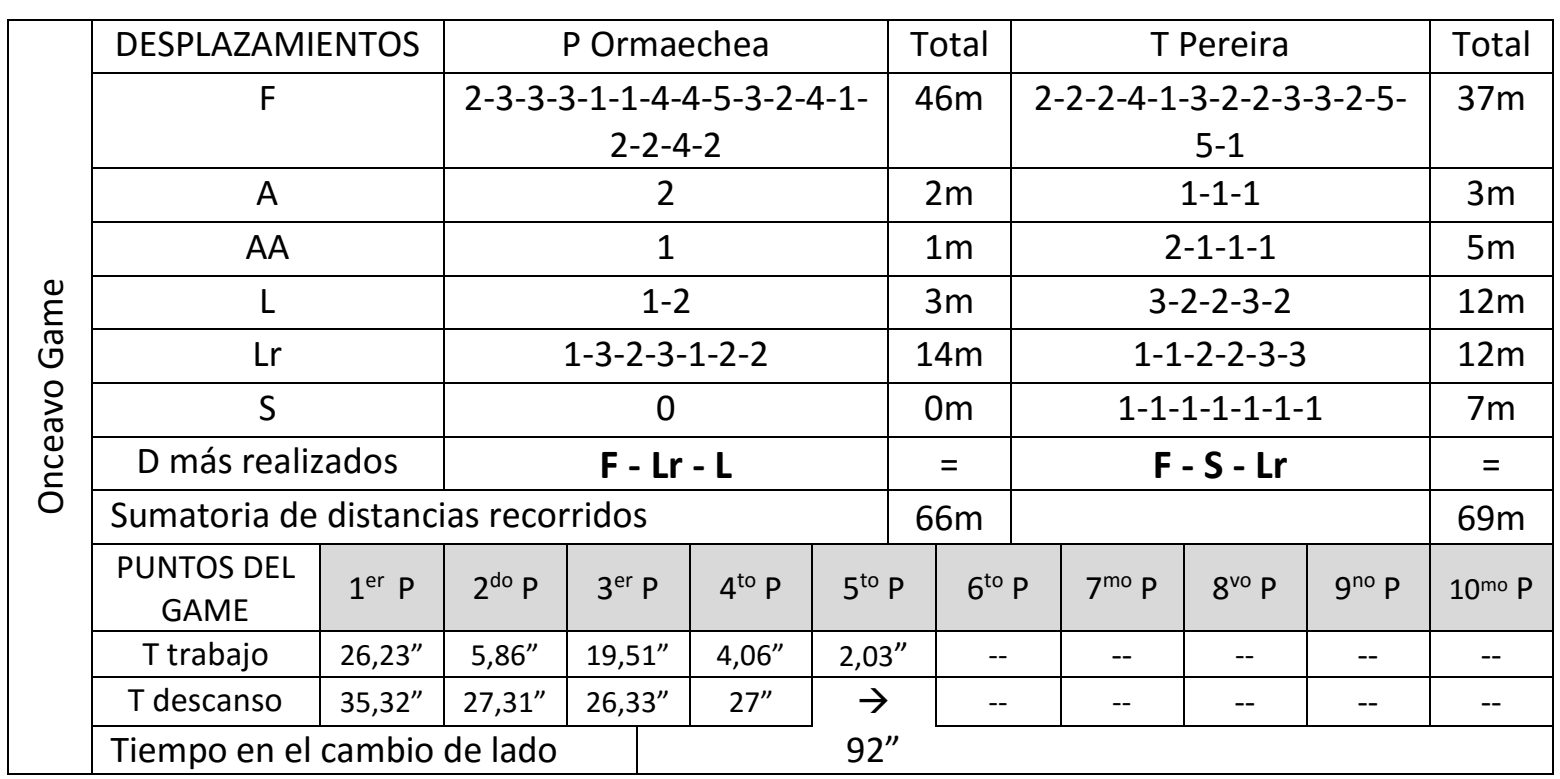

Tabla 53.

\begin{tabular}{|c|c|c|c|c|c|c|c|c|c|c|c|}
\hline \multirow{13}{*}{ 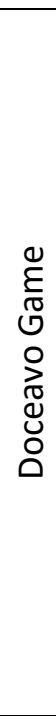 } & \multicolumn{2}{|c|}{ DESPLAZAMIENTOS } & \multicolumn{4}{|c|}{ P Ormaechea } & Total & \multicolumn{3}{|c|}{ T Pereira } & Total \\
\hline & \multicolumn{2}{|c|}{$\mathrm{F}$} & \multicolumn{4}{|c|}{$3-1-3-4-2-2-4-1-1$} & $21 \mathrm{~m}$ & \multicolumn{3}{|c|}{$3-1$} & $4 m$ \\
\hline & \multicolumn{2}{|l|}{ A } & \multicolumn{4}{|c|}{1} & $1 \mathrm{~m}$ & \multicolumn{3}{|c|}{$1-1-1-1-2-2$} & $8 m$ \\
\hline & \multicolumn{2}{|l|}{ AA } & \multicolumn{4}{|c|}{ 1-1-1-1-1-1 } & $6 \mathrm{~m}$ & \multicolumn{3}{|c|}{0} & $0 m$ \\
\hline & \multicolumn{2}{|l|}{$\mathrm{L}$} & \multicolumn{4}{|c|}{$2-1$} & $3 m$ & \multicolumn{3}{|c|}{$1-2$} & $3 m$ \\
\hline & \multicolumn{2}{|l|}{$\mathrm{Lr}$} & \multicolumn{4}{|c|}{$2-1$} & $3 m$ & \multicolumn{3}{|c|}{$2-3-2-2$} & $9 m$ \\
\hline & \multicolumn{2}{|l|}{$S$} & \multicolumn{4}{|c|}{ 1-1-1-1-1-1 } & $0 m$ & \multicolumn{3}{|c|}{0} & $0 m$ \\
\hline & \multicolumn{2}{|c|}{ D más realizados } & \multicolumn{4}{|c|}{$\mathbf{F}-\mathbf{A A}-\mathrm{S}$} & $=$ & \multicolumn{3}{|c|}{ Lr - A - F } & $=$ \\
\hline & \multicolumn{6}{|c|}{ Sumatoria de distancias recorridos } & $34 m$ & & & & $24 \mathrm{~m}$ \\
\hline & $\begin{array}{c}\text { PUNTOS DEL } \\
\text { GAME }\end{array}$ & $1^{\text {er }} P$ & $2^{\text {do }} \mathrm{P}$ & $3^{\text {er }} p$ & $4^{\text {to }} \mathrm{P}$ & $5^{\text {to }} \mathrm{P}$ & $6^{\text {to }} \mathrm{P}$ & $7^{\text {mo }} \mathrm{p}$ & $8^{\text {vo } P}$ & $9^{\text {no }} P$ & $10^{\text {mo }} \mathrm{P}$ \\
\hline & T trabajo & $3,42^{\prime \prime}$ & $3,92^{\prime \prime}$ & $5,82^{\prime \prime}$ & $2,08^{\prime \prime}$ & $18,50^{\prime \prime}$ & -- & -- & -- & -- & -- \\
\hline & T descanso & $18,14^{\prime \prime}$ & $19,79^{\prime \prime}$ & $22,05^{\prime \prime}$ & $16,25^{\prime \prime}$ & $\rightarrow$ & - & -- & -- & -- & -- \\
\hline & Tiempo en e & ambio & de serv & & & $36,73^{\prime \prime}$ & & & & & \\
\hline
\end{tabular}

Tabla 54. 


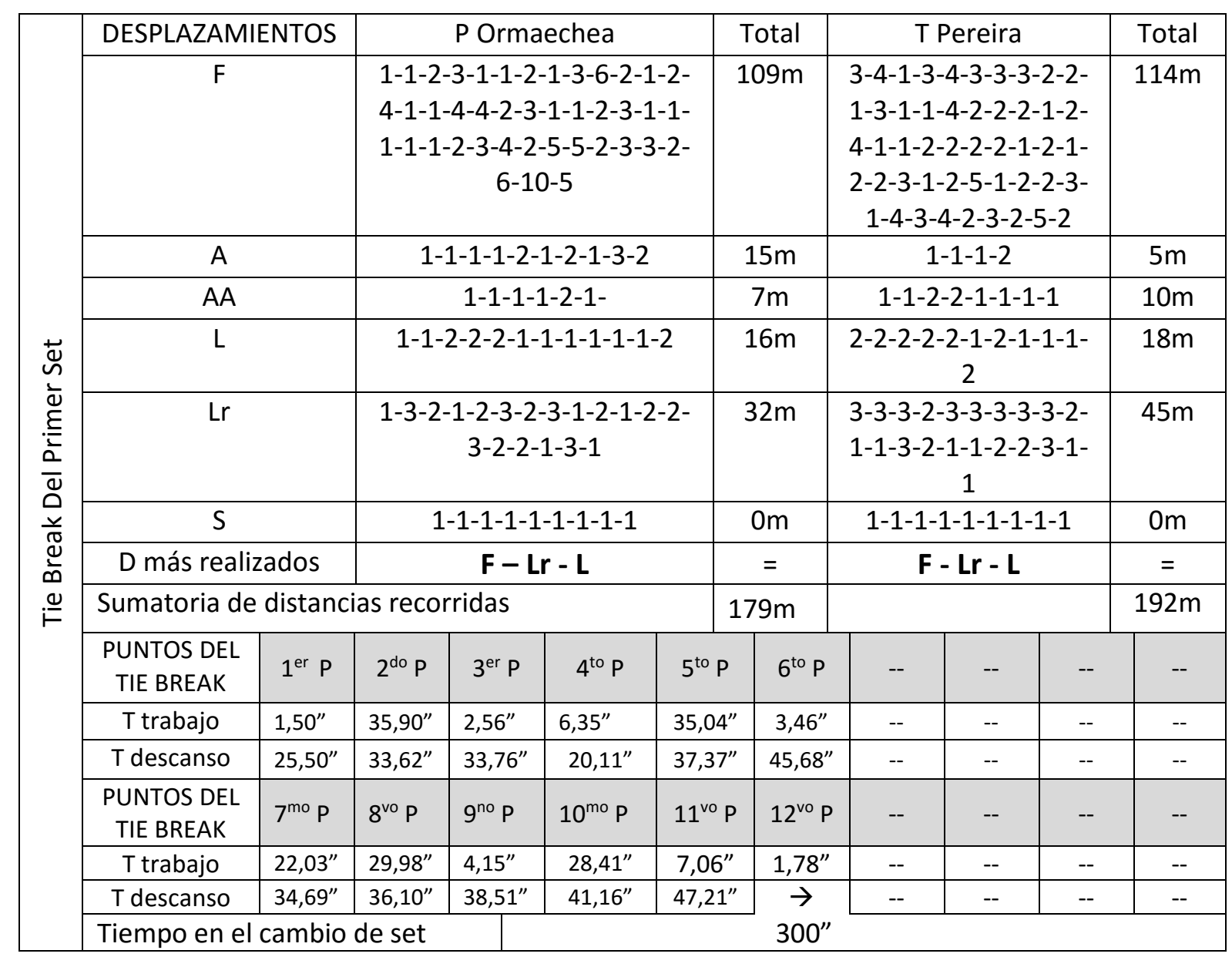

Tabla 55. 


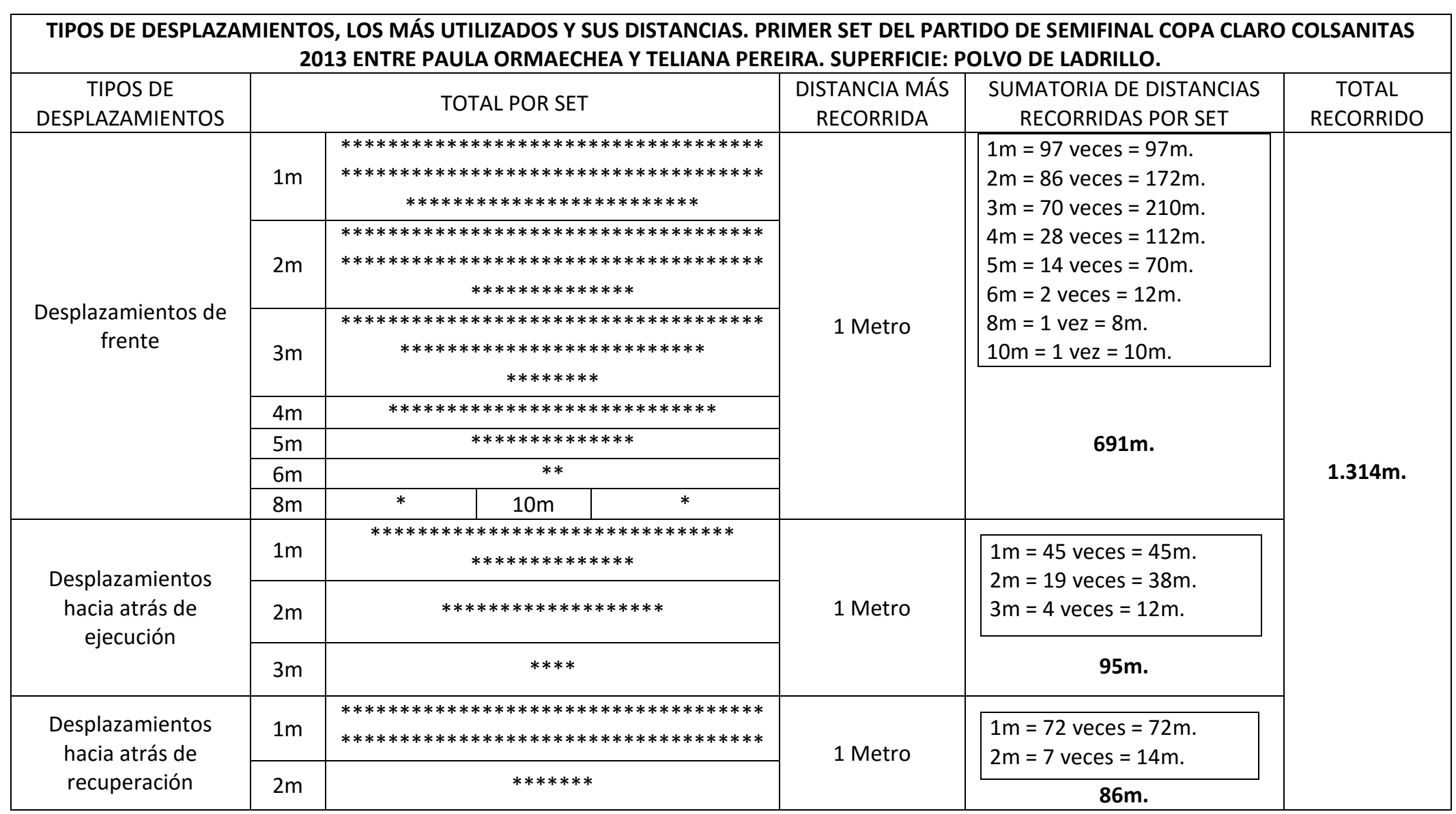

Tabla 56. 


\begin{tabular}{|c|c|c|c|c|c|}
\hline \multicolumn{6}{|c|}{$\begin{array}{l}\text { TIPOS DE DESPLAZAMIENTOS, LOS MÁS UTILIZADOS Y SUS DISTANCIAS. PRIMER SET DEL PARTIDO DE SEMIFINAL COPA CLARO } \\
\text { COLSANITAS2013 ENTRE PAULA ORMAECHEA Y TELIANA PEREIRA. SUPERFICIE: POLVO DE LADRILLO. }\end{array}$} \\
\hline $\begin{array}{c}\text { TIPOS DE } \\
\text { DESPLAZAMIENTOS }\end{array}$ & \multicolumn{2}{|r|}{ TOTAL POR SET } & $\begin{array}{l}\text { DISTANCIA MÁS } \\
\text { RECORRIDA }\end{array}$ & $\begin{array}{l}\text { SUMATORIA DE DISTANCIAS } \\
\text { RECORRIDAS POR SET }\end{array}$ & $\begin{array}{c}\text { TOTAL } \\
\text { RECORRIDO }\end{array}$ \\
\hline \multirow{3}{*}{$\begin{array}{l}\text { Desplazamientos } \\
\text { laterales de ejecución }\end{array}$} & $1 \mathrm{~m}$ & $\begin{array}{c}* * * * * * * * * * * * * * * * * * * * * * * * * * * * * * * * * * * * * \\
* * * * * * *\end{array}$ & \multirow{3}{*}{1 Metro } & \multirow{2}{*}{$\begin{array}{l}1 m=44 \text { veces }=44 m \\
2 m=30 \text { veces }=60 m \\
3 m=5 \text { veces }=15 m\end{array}$} & \multirow{8}{*}{$\uparrow$} \\
\hline & $2 m$ & $* * * * * * * * * * * * * * * * * * * * * * * * * * * * * * *$ & & & \\
\hline & $3 m$ & $* * * * *$ & & $119 \mathrm{~m}$. & \\
\hline \multirow{4}{*}{$\begin{array}{l}\text { Desplazamientos } \\
\text { laterales de } \\
\text { recuperación. }\end{array}$} & $1 \mathrm{~m}$ & $\begin{array}{c}* * * * * * * * * * * * * * * * * * * * * * * * * * * * * * * * * * * * * \\
* * * *\end{array}$ & \multirow{4}{*}{2 Metros } & \multirow{2}{*}{$\begin{array}{l}1 \mathrm{~m}=41 \text { veces }=41 \mathrm{~m} \\
2 \mathrm{~m}=65 \text { veces }=130 \mathrm{~m} \\
3 \mathrm{~m}=48 \text { veces }=144 \mathrm{~m} \\
4 \mathrm{~m}=2 \text { veces }=8 \mathrm{~m}\end{array}$} & \\
\hline & $2 m$ & $\begin{array}{c}* * * * * * * * * * * * * * * * * * * * * * * * * * * * * * * * * * * * * \\
* * * * * * * * * * * * * * * * * * * * * * * * * * * *\end{array}$ & & & \\
\hline & $3 m$ & $\begin{array}{c}* * * * * * * * * * * * * * * * * * * * * * * * * * * * * * * * * * * * *) \\
* * * * * * * * * * *\end{array}$ & & \multirow{2}{*}{$323 \mathrm{~m}$. } & \\
\hline & $4 m$ & $* *$ & & & \\
\hline $\begin{array}{l}\text { Desplazamientos } \\
\text { hacia arriba o saltos }\end{array}$ & \multicolumn{2}{|c|}{$\begin{array}{c}* * * * * * * * * * * * * * * * * * * * * * * * * * * * * * * * * * * * * * * * * * \\
* * * * * * * * * * * * * * * * * * * * * * * * * * * * * * * * * * * * * * * * * \\
* * * * * * * * *\end{array}$} & \multicolumn{2}{|c|}{ Saltos en servicio $=93$ veces } & \\
\hline
\end{tabular}

Tabla 57.

\begin{tabular}{|c|c|c|c|c|c|c|}
\hline \multicolumn{7}{|c|}{$\begin{array}{l}\text { PORCENTAJES DE USO DE LOS DIFERENTES TIPOS DE DESPLAZAMIENTOS EN EL PRIMER SET DE LA SEMIFINAL COPA CLARO COLSANITAS2013 } \\
\text { ENTRE PAULA ORMAECHEA Y TELIANA PEREIRA. SUPERFICIE: POLVO DE LADRILLO. }\end{array}$} \\
\hline & $\begin{array}{l}\text { D HACIA } \\
\text { ADELANTE }\end{array}$ & $\begin{array}{l}\text { D HACIA ATRÁS } \\
\text { DE EJECUCIÓN }\end{array}$ & $\begin{array}{l}\text { D HACIA ATRÁS DE } \\
\text { RECUPERACIÓN }\end{array}$ & $\begin{array}{l}\text { D LATERALES } \\
\text { DE EJECUCIÓN }\end{array}$ & $\begin{array}{l}\text { D LATERALES DE } \\
\text { RECUPERACIÓN }\end{array}$ & $\begin{array}{l}\text { D HACIA } \\
\text { ARRIBA }\end{array}$ \\
\hline NÚMERO DE VECES & $38,69 \%$ & $8,78 \%$ & $10,20 \%$ & $10,20 \%$ & $20,15 \%$ & $12,01 \%$ \\
\hline DISTANCIA & $52,34 \%$ & $7,19 \%$ & $6,51 \%$ & $9,01 \%$ & $24,92 \%$ & \\
\hline
\end{tabular}

Tabla 58. 


\begin{tabular}{|c|c|c|c|c|}
\hline \multicolumn{5}{|c|}{$\begin{array}{l}\text { TIEMPOS GLOBALES DE LOS MOMENTOS DE TRABAJO Y PAUSA DEL PRIMER SET DEL PARTIDO ENTRE } \\
\text { PAULA ORMAECHEA vs TELIANA PEREIRA, SEMIFINAL COPA CLARO COLSANITAS } 2013 .\end{array}$} \\
\hline TIEMPOS & $\begin{array}{l}\text { SUMATO } \\
\text { TIEMPOS P }\end{array}$ & $\begin{array}{l}\text { R GA DE } \\
\text { R GAME }\end{array}$ & $\begin{array}{l}\text { SUMATORIA DE TIEMPOS } \\
\text { POR SET }\end{array}$ & $\begin{array}{l}\text { RELACIÓN TIEMPO } \\
\text { DE TRABAJO PAUSA }\end{array}$ \\
\hline \multirow{13}{*}{ TIEMPO DE TRABAJO } & $1^{\text {er }}$ Game & $51,02^{\prime \prime}$ & \multirow{13}{*}{$\begin{array}{l}668,17 " \text { tiempo real de } \\
\text { trabajo }\end{array}$} & \multirow{26}{*}{$\begin{array}{c}\text { 1" de Trabajo. } \\
x \\
3,62 " \text { de Pausa. }\end{array}$} \\
\hline & $2^{\text {do }}$ Game & $45,93^{\prime \prime}$ & & \\
\hline & $3^{\text {er }}$ Game & $27,54^{\prime \prime}$ & & \\
\hline & $4^{\text {to }}$ Game & $35,74^{\prime \prime}$ & & \\
\hline & $5^{\text {to }}$ Game & $57,07^{\prime \prime}$ & & \\
\hline & $6^{\text {to }}$ Game & $57,97 "$ & & \\
\hline & $7^{\text {to }}$ Game & $30,17^{\prime \prime}$ & & \\
\hline & $8^{\text {vo }}$ Game & $28,45^{\prime \prime}$ & & \\
\hline & $9^{\text {no }}$ Game & $26,7^{\prime \prime}$ & & \\
\hline & $10^{\text {mo }}$ Game & $37,93^{\prime \prime}$ & & \\
\hline & $11^{\text {vo }}$ Game & $57,69^{\prime \prime}$ & & \\
\hline & $12^{\text {vo }}$ Game & $33,74^{\prime \prime}$ & & \\
\hline & Tie Break & $178,22^{\prime \prime}$ & & \\
\hline \multirow{13}{*}{ TIEMPO DE DESCANSO } & $1^{\mathrm{er}}$ Game & $130,78^{\prime \prime}$ & \multirow{13}{*}{$\begin{array}{l}2.421,9^{\prime \prime} \text { Tiempo real de } \\
\text { descanso }\end{array}$} & \\
\hline & $2^{\text {do }}$ Game & $139,3^{\prime \prime}$ & & \\
\hline & $3^{\text {er }}$ Game & $170,06^{\prime \prime}$ & & \\
\hline & $4^{\text {to }}$ Game & $129,71^{\prime \prime}$ & & \\
\hline & $5^{\text {to }}$ Game & $178,35^{\prime \prime}$ & & \\
\hline & $6^{\text {to }}$ Game & $170,72^{\prime \prime}$ & & \\
\hline & $7^{\text {to }}$ Game & $161,97^{\prime \prime}$ & & \\
\hline & $8^{\text {vo }}$ Game & $106,21^{\prime \prime}$ & & \\
\hline & $9^{\text {no }}$ Game & $155,63^{\prime \prime}$ & & \\
\hline & $10^{\text {mo }}$ Game & $119,54^{\prime \prime}$ & & \\
\hline & $11^{\text {vo }}$ Game & $207,96 "$ & & \\
\hline & $12^{\text {vo }}$ Game & $112,96 "$ & & \\
\hline & Tie Break & $638,71^{\prime \prime}$ & & \\
\hline
\end{tabular}

Tabla 59. 


\begin{tabular}{|c|c|c|c|}
\hline \multicolumn{4}{|c|}{$\begin{array}{l}\text { PROMEDIO DE LOS MOMENTOS ESPECÍFICOS DE TRABAJO Y PAUSA DEL PRIMER SET DEL PARTIDO } \\
\text { ENTRE PAULA ORMAECHEA VS TELIANA PEREIRA, SEMIFINAL COPA CLARO COLSANITAS } 2013 .\end{array}$} \\
\hline \multicolumn{2}{|c|}{ PROMEDIOS DE TRABAJO } & \multicolumn{2}{|c|}{ PROMEDIOS DE PAUSA } \\
\hline $1^{\text {er }}$ Game & $12,75^{\prime \prime}$ & $1^{\text {er }}$ Game & $25,48^{\prime \prime}$ \\
\hline $2^{\text {do }}$ Game & $7,65^{\prime \prime}$ & $2^{\text {do }}$ Game & $21,36^{\prime \prime}$ \\
\hline $3^{\text {er }}$ Game & $6,88^{\prime \prime}$ & $3^{\text {er }}$ Game & $24,35^{\prime \prime}$ \\
\hline $4^{\text {to }}$ Game & $7,14^{\prime \prime}$ & $4^{\text {to }}$ Game & $23,45^{\prime \prime}$ \\
\hline $5^{\text {to }}$ Game & 7,03" & $5^{\text {to }}$ Game & $24,9^{\prime \prime}$ \\
\hline $6^{\text {to }}$ Game & $9,66^{\prime \prime}$ & $6^{\text {to }}$ Game & $26,16^{\prime \prime}$ \\
\hline $7^{\mathrm{mo}}$ Game & 7,54" & $7^{\mathrm{mo}}$ Game & $23,32^{\prime \prime}$ \\
\hline $8^{\text {vo }}$ Game & $7,11^{\prime \prime}$ & $8^{\text {vo }}$ Game & $23,15^{\prime \prime}$ \\
\hline $9^{\text {no }}$ Game & 6,67" & $9^{\text {No }}$ Game & $21,87^{\prime \prime}$ \\
\hline $10^{\mathrm{mo}}$ Game & $7,58^{\prime \prime}$ & $10^{\mathrm{mo}}$ Game & $21,59^{\prime \prime}$ \\
\hline $11^{\text {vo }}$ Game & $11,52^{\prime \prime}$ & $11^{\text {vo }}$ Game & $28,99^{\prime \prime}$ \\
\hline $12^{\text {vo }}$ Game & $6,74^{\prime \prime}$ & $12^{\text {vo }}$ Game & $19,05^{\prime \prime}$ \\
\hline Tie Break & $14,85^{\prime \prime}$ & Tie Break & $34,80^{\prime \prime}$ \\
\hline \multicolumn{2}{|c|}{ Promedio total } & \multicolumn{2}{|c|}{ Promedio total } \\
\hline \multicolumn{2}{|c|}{$8,70^{\prime \prime}$} & \multicolumn{2}{|c|}{$24,49^{\prime \prime}$} \\
\hline \multicolumn{4}{|c|}{ 1" de trabajo por $2,81^{\prime \prime}$ de pausa } \\
\hline
\end{tabular}

Tabla 60.

4. Análisis punto a punto del primer set del partido de la final Master 1000 de Monte-Carlo Rolex Masters 2013 entre Rafael Nadal (Esp) vs Novak Djokovic (Serb). En el que N Djokovic derrotó 6-2 7-6(7-1) a R Nadal. superficie de la cancha (polvo de ladrillo), clasificación ITF categoría 1 (velocidad baja). Ver tablas 62 a 74.

- Análisis de los tiempos y tipos de golpes ejecutados dentro de la cancha antes de comenzar el partido (peloteo previo al partido):

Los jugadores después del acto protocolario, comienzan a pelotear desde el fondo de la cancha a una intensidad media para entrar en ritmo de juego. Al minuto 2' N Djokovic se acerca a dos pasos de la red para comenzar a volear hasta el minuto 2,30', luego se sitúa sobre la "T" para hacer Smash hasta el minuto 2,40' y en ese momento se intercambian los roles y $R$ Nadal pasa ahora a volear cerca a la red hasta el minuto 3,08', para luego situarse sobre la "T" y hacer Smash hasta el minuto 3,20'. Después de esto los jugadores se sitúan detrás de la línea de fondo para hacer servicios o saques hasta el minuto 5,30' 
y, por último, descansan 45" sentados y se alistan para empezar el partido. Ver tabla 61.

\begin{tabular}{|c|c|c|c|c|}
\hline \multicolumn{6}{|c|}{$\begin{array}{c}\text { ANÁLISIS DEL USO DE LOS TIPOS DE GOLPES EN MOMENTO PREVIO AL COMIENZO } \\
\text { DEL PARTIDO(PELOTEO PREVIO AL PARTIDO) }\end{array}$} \\
\hline Tipo de golpe & $\begin{array}{c}\text { N Djokovic } \\
\text { R Nadal }\end{array}$ & Tiempo promedio & $\%$ \\
\hline $\begin{array}{c}\text { (derecha y revés) Peloteo } \\
\text { de fondo de cancha }\end{array}$ & $\begin{array}{c}120 "+28 "+12 " \\
=160\end{array}$ & $160 "$ & $160 "$ & $48,48 \%$ \\
\hline Volea cerca a la red & $30 "$ & $28 ”$ & $29 "$ & $8,78 \%$ \\
\hline Smash sobre la "T" & $10 "$ & $12 "$ & $11 "$ & $3,33 \%$ \\
\hline Servicio o saque & $130 "$ & $130 "$ & $130 "$ & $39,39 \%$ \\
\hline
\end{tabular}

Tabla 61.

\begin{tabular}{|c|c|c|c|c|c|c|c|c|c|c|c|}
\hline \multirow{13}{*}{ 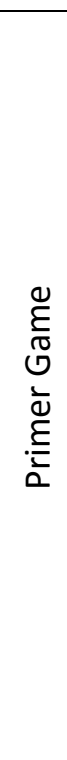 } & \multicolumn{2}{|c|}{ DESPLAZAMIENTOS } & \multicolumn{4}{|c|}{ N Djokovic } & Total & \multicolumn{3}{|c|}{ R NADAL } & Total \\
\hline & \multicolumn{2}{|c|}{$\mathrm{F}$} & \multicolumn{4}{|c|}{$4-2-2-3-2-3-3-2-2-4$} & $27 \mathrm{~m}$ & \multicolumn{3}{|c|}{$\begin{array}{c}2-4-2-1-2-2-6-6-4- \\
1-3-1\end{array}$} & $34 m$ \\
\hline & \multicolumn{2}{|l|}{ A } & \multicolumn{4}{|c|}{0} & $0 m$ & \multicolumn{3}{|c|}{2} & $2 m$ \\
\hline & \multicolumn{2}{|l|}{$A A$} & \multicolumn{4}{|c|}{ 1-1-1-1 } & $4 m$ & \multicolumn{3}{|c|}{0} & $0 m$ \\
\hline & \multicolumn{2}{|l|}{$\mathrm{L}$} & \multicolumn{4}{|c|}{1} & $1 \mathrm{~m}$ & \multicolumn{3}{|c|}{1} & $1 \mathrm{~m}$ \\
\hline & \multicolumn{2}{|l|}{ Lr } & \multicolumn{4}{|c|}{$3-1-2-2-2-1$} & $11 \mathrm{~m}$ & \multicolumn{3}{|c|}{$1-2-1-2-1-2-1$} & $10 m$ \\
\hline & \multicolumn{2}{|l|}{$S$} & \multicolumn{4}{|c|}{ 1-1-1-1-1-1-1 } & $0 m$ & \multicolumn{3}{|c|}{0} & $0 m$ \\
\hline & \multicolumn{2}{|c|}{ D más realizados } & \multicolumn{4}{|c|}{$F-S-L r$} & $=$ & \multicolumn{3}{|c|}{$F-L r-A$} & $=$ \\
\hline & \multicolumn{6}{|c|}{ Sumatoria de distancias recorridos } & $43 m$ & & & & $47 m$ \\
\hline & $\begin{array}{l}\text { PUNTOS DEL } \\
\text { GAME }\end{array}$ & $1^{\text {er }} \mathrm{P}$ & $2^{\text {do }} \mathrm{P}$ & $3^{\text {er }} \mathrm{P}$ & $4^{\text {to }} \mathrm{P}$ & $5^{\text {to }} \mathrm{P}$ & $6^{\text {to }} \mathrm{P}$ & $7^{\mathrm{mo}} \mathrm{P}$ & $8^{\text {vo }} \mathrm{P}$ & $9^{\text {no }} P$ & $10^{\text {mo }} \mathrm{P}$ \\
\hline & T trabajo & $5,38^{\prime \prime}$ & $14,38^{\prime \prime}$ & $14,33^{\prime \prime}$ & $2,35^{\prime \prime}$ & $1,09^{\prime \prime}$ & -- & -- & -- & -- & -- \\
\hline & T descanso & $18,76^{\prime \prime}$ & $20,99^{\prime \prime}$ & $25,36^{\prime \prime}$ & $22,48^{\prime \prime}$ & $\rightarrow$ & -- & -- & -- & -- & -- \\
\hline & \multicolumn{6}{|c|}{ Tiempo en el cambio de lado } & & & & & \\
\hline
\end{tabular}

Tabla 62 


\begin{tabular}{|c|c|c|c|c|c|c|c|c|c|c|c|}
\hline & \multicolumn{2}{|c|}{ DESPLAZAMIENTOS } & \multicolumn{4}{|c|}{ N Djokovic } & Total & \multicolumn{3}{|c|}{ R NADAL } & Total \\
\hline & \multicolumn{2}{|l|}{$\mathrm{F}$} & \multicolumn{4}{|c|}{$\begin{array}{c}2-2-2-3-2-2-2-1-2-1-3-2-3- \\
1-1-3-1-4-2-1-4-2-3-3-3-2- \\
1-1-3-6-4-5-4-4-1-1-6-1-4- \\
5-2-2-2-6-2-2-2-3-1-4-2\end{array}$} & $131 \mathrm{~m}$ & \multicolumn{3}{|c|}{$\begin{array}{c}1-3-4-2-4-3-1-1-2- \\
3-3-1-4-5-4-1-2-3- \\
3-5-4-2-9-4-3-2-2- \\
1-4-2-3-3-4-4-4\end{array}$} & $106 m$ \\
\hline & \multicolumn{2}{|l|}{ A } & \multicolumn{4}{|c|}{$2-2$} & $4 m$ & \multicolumn{3}{|c|}{$3-3-3-1-3-4-3-3-3$} & $26 m$ \\
\hline & \multicolumn{2}{|l|}{ AA } & \multicolumn{4}{|c|}{2} & $2 m$ & \multicolumn{3}{|c|}{$1-1-1-1-1-1-1$} & $7 m$ \\
\hline & \multicolumn{2}{|l|}{$\mathrm{L}$} & \multicolumn{4}{|c|}{$3-1-1-1$} & $6 m$ & \multicolumn{3}{|c|}{$2-1-1-1-1-1$} & $7 \mathrm{~m}$ \\
\hline & \multicolumn{2}{|l|}{$\mathrm{Lr}$} & \multicolumn{4}{|c|}{$\begin{array}{c}1-1-2-2-2-2-1-2-1-3-2-2-3- \\
1-1-1-3-2-2-1-3-2-2-2-1-3- \\
2-1-1-3\end{array}$} & $55 \mathrm{~m}$ & \multicolumn{3}{|c|}{$\begin{array}{l}1-2-2-3-1-1-1-3-2- \\
3-3-2-2-3-2-2-1-3- \\
2-1-3-2-2-3-3-2-3\end{array}$} & $58 m$ \\
\hline & \multicolumn{2}{|l|}{ S } & \multicolumn{4}{|c|}{0} & $0 \mathrm{~m}$ & \multicolumn{3}{|c|}{$\begin{array}{c}\text { 1-1-1-1-1-1-1-1-1- } \\
\text { 1-1-1-1-1-1-1 }\end{array}$} & $0 m$ \\
\hline & \multicolumn{2}{|c|}{ D más realizados } & \multicolumn{4}{|c|}{$F-\mathbf{L r}-\mathbf{L}$} & $=$ & \multicolumn{3}{|c|}{$F-\operatorname{Lr}-\mathbf{S}$} & $=$ \\
\hline & \multicolumn{6}{|c|}{ Sumatoria de distancias recorridos } & $196 \mathrm{~m}$ & & & & $167 \mathrm{~m}$ \\
\hline & $\begin{array}{c}\text { PUNTOS DEL } \\
\text { GAME }\end{array}$ & $1^{\text {er }} \mathrm{P}$ & $2^{\text {do }} \mathrm{P}$ & $3^{\text {er }} \mathrm{P}$ & $4^{\text {to }} \mathrm{P}$ & $5^{\text {to }} \mathrm{P}$ & $6^{\text {to }} \mathrm{P}$ & $7^{\mathrm{mo}} \mathrm{P}$ & $8^{\text {vo }} \mathrm{P}$ & $9^{\text {no }} P$ & $10^{\mathrm{mo}} \mathrm{P}$ \\
\hline & T trabajo & $35,45^{\prime \prime}$ & $1,35^{\prime \prime}$ & $4,60^{\prime \prime}$ & $4,27 "$ & $19,56^{\prime \prime}$ & $20,90^{\prime \prime}$ & $17,45^{\prime \prime}$ & $2,82^{\prime \prime}$ & $4,56^{\prime \prime}$ & $12,58^{\prime \prime}$ \\
\hline & T descanso & $26,65^{\prime \prime}$ & $23^{\prime \prime}$ & $20,55^{\prime \prime}$ & $22,13^{\prime \prime}$ & $30^{\prime \prime}$ & $27,66^{\prime \prime}$ & $28,38^{\prime \prime}$ & $22,39 \prime$ & $24,73^{\prime \prime}$ & $22,75^{\prime \prime}$ \\
\hline & $\begin{array}{c}\text { PUNTOS DEL } \\
\text { GAME }\end{array}$ & $11^{\text {vo }} \mathrm{P}$ & $12^{\text {vo }} \mathrm{P}$ & $13^{\text {vo }} \mathrm{P}$ & $14^{\text {vo }} \mathrm{P}$ & $15^{\mathrm{vo}} \mathrm{P}$ & $16^{\text {vo }} \mathrm{P}$ & $17^{\mathrm{vo}} \mathrm{P}$ & $18^{\text {vo }} \mathrm{P}$ & $19^{\text {vo }} \mathrm{P}$ & $20^{\text {vo }} \mathrm{P}$ \\
\hline & T trabajo & $11,51^{\prime \prime}$ & $12,41^{\prime \prime}$ & -- & -- & -- & -- & -- & -- & -- & -- \\
\hline & T descanso & $27,74^{\prime \prime}$ & $\rightarrow$ & -- & -- & -- & -- & -- & -- & -- & -- \\
\hline & \multicolumn{4}{|c|}{$30,06 "$} & \multicolumn{6}{|c|}{ Tiempo en el cambio de servicio } & \\
\hline
\end{tabular}

\section{Tabla 63.}




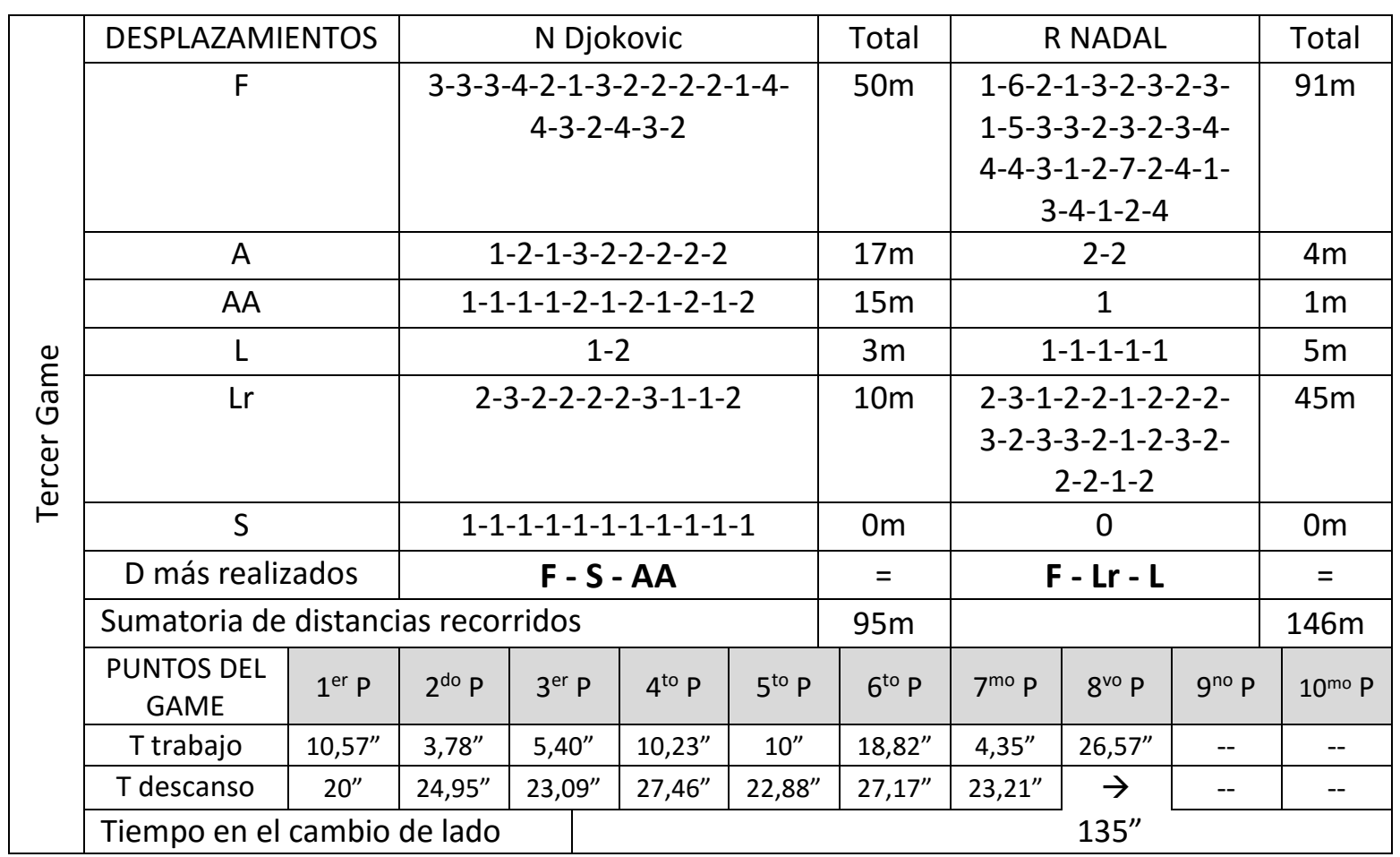

Tabla 64.

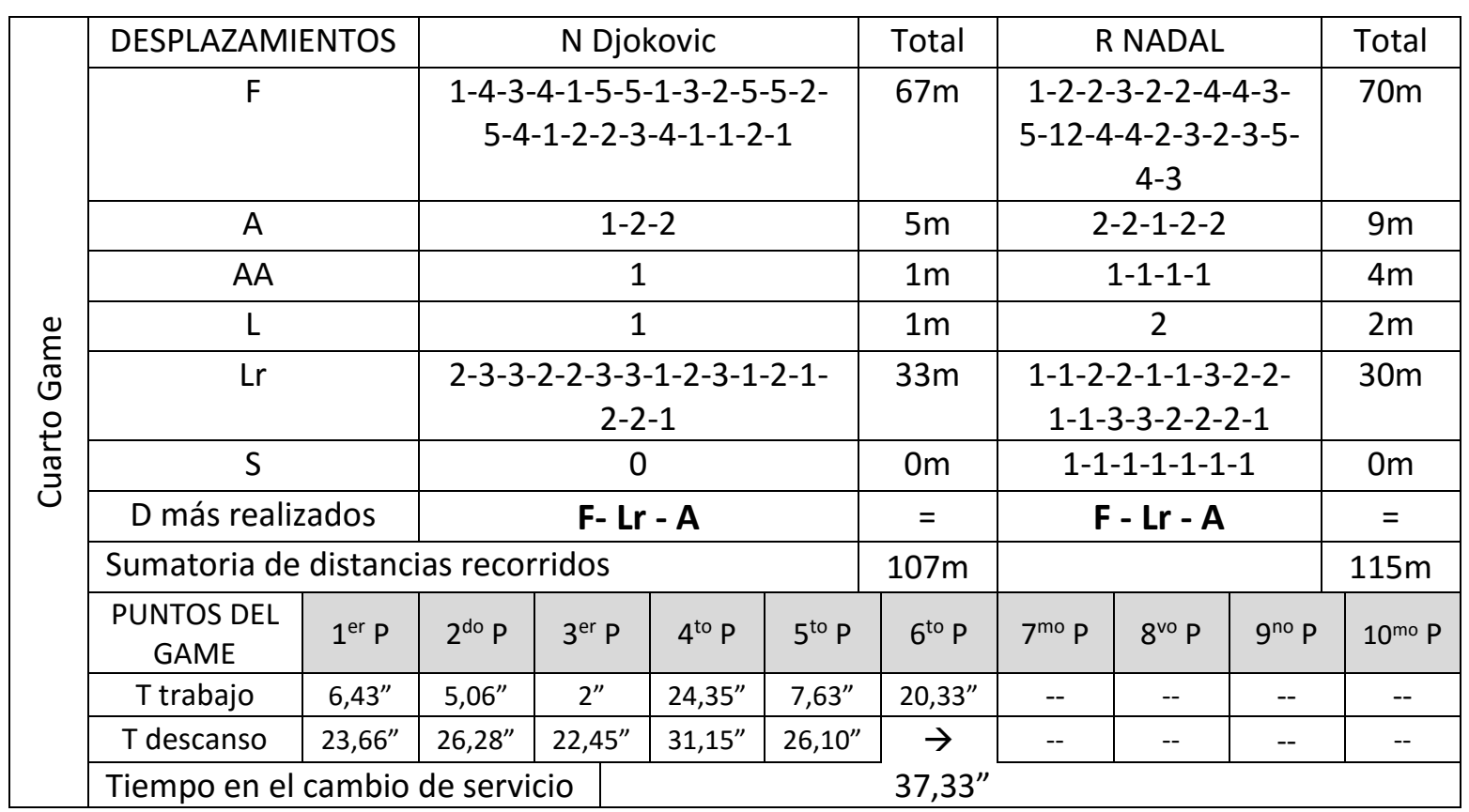

\section{Tabla 65.}




\begin{tabular}{|c|c|c|c|c|c|c|c|c|c|c|c|}
\hline \multirow{13}{*}{ 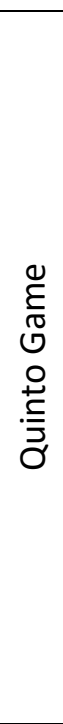 } & \multicolumn{2}{|c|}{ DESPLAZAMIENTOS } & \multicolumn{4}{|c|}{ N Djokovic } & Total & \multicolumn{3}{|c|}{ R NADAL } & Total \\
\hline & \multicolumn{2}{|l|}{$\mathrm{F}$} & \multicolumn{4}{|c|}{$2-5-3-2$} & $12 m$ & \multicolumn{3}{|c|}{$3-1-3-2-12-1-2-7-6$} & $37 m$ \\
\hline & \multicolumn{2}{|l|}{ A } & \multicolumn{4}{|c|}{0} & $0 m$ & \multicolumn{3}{|c|}{0} & $0 m$ \\
\hline & \multicolumn{2}{|l|}{ AA } & \multicolumn{4}{|c|}{ 1-1-1 } & $3 m$ & \multicolumn{3}{|c|}{0} & Om \\
\hline & \multicolumn{2}{|l|}{$\mathrm{L}$} & \multicolumn{4}{|c|}{2} & $2 m$ & \multicolumn{3}{|c|}{0} & Om \\
\hline & \multicolumn{2}{|l|}{ Lr } & \multicolumn{4}{|c|}{2} & $2 m$ & \multicolumn{3}{|c|}{$1-1$} & $2 m$ \\
\hline & \multicolumn{2}{|l|}{$S$} & \multicolumn{4}{|c|}{ 1-1-1-1-1-1 } & $0 m$ & \multicolumn{3}{|c|}{0} & Om \\
\hline & \multicolumn{2}{|c|}{ D más realizados } & \multicolumn{4}{|c|}{$S-F-A A$} & $=$ & \multicolumn{3}{|c|}{ F- Lr } & $=$ \\
\hline & \multicolumn{6}{|c|}{ Sumatoria de distancias recorridos } & $19 m$ & & & & $39 m$ \\
\hline & $\begin{array}{c}\text { PUNTOS DEL } \\
\text { GAME }\end{array}$ & $1^{\text {er }} \mathrm{p}$ & $2^{\text {do }} \mathrm{P}$ & $3^{\text {er } P}$ & $4^{\text {to }} \mathrm{P}$ & $5^{\text {to }} \mathrm{P}$ & $6^{\text {to }} P$ & $7^{\mathrm{mo}} \mathrm{P}$ & $8^{\text {vo } P}$ & $9^{\text {no }} P$ & $10^{\text {mo }} P$ \\
\hline & T trabajo & $2,68^{\prime \prime}$ & $5.35^{\prime \prime}$ & $4,87^{\prime \prime}$ & $7^{\prime \prime}$ & $1,53^{\prime \prime}$ & -- & -- & -- & -- & -- \\
\hline & T descanso & $18,48^{\prime \prime}$ & $23,09^{\prime \prime}$ & $26,55^{\prime \prime}$ & $20,60^{\prime \prime}$ & $\rightarrow$ & -- & -- & -- & -- & -- \\
\hline & \multicolumn{5}{|c|}{ Tiempo en el cambio de lado } & $131^{\prime \prime}$ & & & & & \\
\hline
\end{tabular}

\section{Tabla 66.}

\begin{tabular}{|c|c|c|c|c|c|c|c|c|c|c|c|}
\hline & \multicolumn{2}{|c|}{ DESPLAZAMIENTOS } & \multicolumn{4}{|c|}{ N Djokovic } & Total & \multicolumn{3}{|c|}{ R NADAL } & Total \\
\hline & \multicolumn{2}{|l|}{$\mathrm{F}$} & \multicolumn{4}{|c|}{$\begin{array}{c}3-4-1-1-2-2-3-3-2-3-6-3-4- \\
1-2-6-2-2-1-7-6-4-1-1-3-1- \\
2-4-3-6-2-2-3-5-6-1-8-6\end{array}$} & $122 m$ & \multicolumn{3}{|c|}{$\begin{array}{l}2-1-3-3-1-3-1-3-1-5- \\
6-1-1-4-4-4-2-4-5-3\end{array}$} & $57 m$ \\
\hline & \multicolumn{2}{|l|}{ A } & \multicolumn{4}{|c|}{2} & $2 m$ & \multicolumn{3}{|c|}{$1-3-2-3-2-2-2-1-1$} & $17 m$ \\
\hline & \multicolumn{2}{|l|}{$\mathrm{AA}$} & \multicolumn{4}{|c|}{0} & $0 m$ & \multicolumn{3}{|c|}{ 1-1-1-1-1-1-1 } & $7 m$ \\
\hline & \multicolumn{2}{|l|}{$L$} & \multicolumn{4}{|c|}{$2-3-1$} & $6 m$ & \multicolumn{3}{|c|}{$2-2-2-2-1$} & $9 m$ \\
\hline & \multicolumn{2}{|l|}{$\mathrm{Lr}$} & \multicolumn{4}{|c|}{$\begin{array}{c}2-3-3-1-1-3-3-3-3-1-2-2-1- \\
3-1-2-3-2-3\end{array}$} & $42 m$ & \multicolumn{3}{|c|}{$\begin{array}{c}2-2-2-2-2-3-2-1-1-3- \\
2-2-3-3\end{array}$} & $30 \mathrm{~m}$ \\
\hline 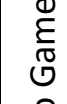 & \multicolumn{2}{|l|}{$S$} & \multicolumn{4}{|c|}{0} & $0 \mathrm{~m}$ & \multicolumn{3}{|c|}{$\begin{array}{c}\text { 1-1-1-1-1-1-1-1-1-1- } \\
\text { 1-1-1-1-1-1 } \\
\end{array}$} & $0 \mathrm{~m}$ \\
\hline$\underset{x}{+}$ & \multicolumn{2}{|c|}{ D más realizados } & \multicolumn{4}{|c|}{$F-L r-L$} & $=$ & \multicolumn{3}{|c|}{$F-S-L r$} & $=$ \\
\hline & \multicolumn{6}{|c|}{ Sumatoria de distancias recorridas } & $172 m$ & \multicolumn{4}{|r|}{$120 \mathrm{~m}$} \\
\hline & $\begin{array}{l}\text { PUNTOS DEL } \\
\text { GAME }\end{array}$ & $1^{\text {er } P}$ & $2^{\text {do }} P$ & $3^{\text {er }} \mathrm{P}$ & $4^{\text {to } P}$ & $5^{\text {to }} \mathrm{P}$ & $6^{\text {to }} P$ & $7^{\text {mo }} \mathrm{P}$ & $8^{\text {vo } P}$ & $9^{\text {no }} P$ & $10^{\text {mo }} P$ \\
\hline & T trabajo & $5,06 "$ & $7,53^{\prime \prime}$ & $13,58^{\prime \prime}$ & $5,52^{\prime \prime}$ & $8,23^{\prime \prime}$ & $2,33^{\prime \prime}$ & $16,33^{\prime \prime}$ & $2,25^{\prime \prime}$ & $8,62^{\prime \prime}$ & $3,10^{\prime \prime}$ \\
\hline & T descanso & $22,18^{\prime \prime}$ & $24,80^{\prime \prime}$ & $25,28^{\prime \prime}$ & $22,13 "$ & $24,10^{\prime}$ & 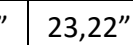 & $20,78^{\prime \prime}$ & $27,34^{\prime \prime}$ & $20,33^{\prime \prime}$ & $21,75^{\prime \prime}$ \\
\hline & $\begin{array}{c}\text { PUNTOS DEL } \\
\text { GAME }\end{array}$ & $11^{\text {vo }} \mathrm{P}$ & $12^{\mathrm{vo}} \mathrm{P}$ & $13^{\text {vo }} \mathrm{P}$ & $14^{\text {vo }} \mathrm{P}$ & $15^{\mathrm{vo}} \mathrm{P}$ & $16^{\text {vo }} \mathrm{P}$ & $17^{\text {vo }} \mathrm{P}$ & $18^{\text {vo }} \mathrm{P}$ & $19^{\text {vo }} \mathrm{P}$ & $20^{\text {vo }} \mathrm{P}$ \\
\hline & $\mathrm{T}$ trabajo & $18,23^{\prime \prime}$ & $2,61^{\prime \prime}$ & $5,90^{\prime \prime}$ & $7,51^{\prime \prime}$ & -- & -- & -- & -- & -- & -- \\
\hline & T descanso & $20,61^{\prime \prime}$ & $25,04^{\prime \prime}$ & $26,32^{\prime \prime}$ & $\rightarrow$ & -- & -- & -- & -- & -- & -- \\
\hline & Tiempo en $\epsilon$ & ambic & e serv & & $40,47^{\prime \prime}$ & & & & & & \\
\hline
\end{tabular}

\section{Tabla 67.}




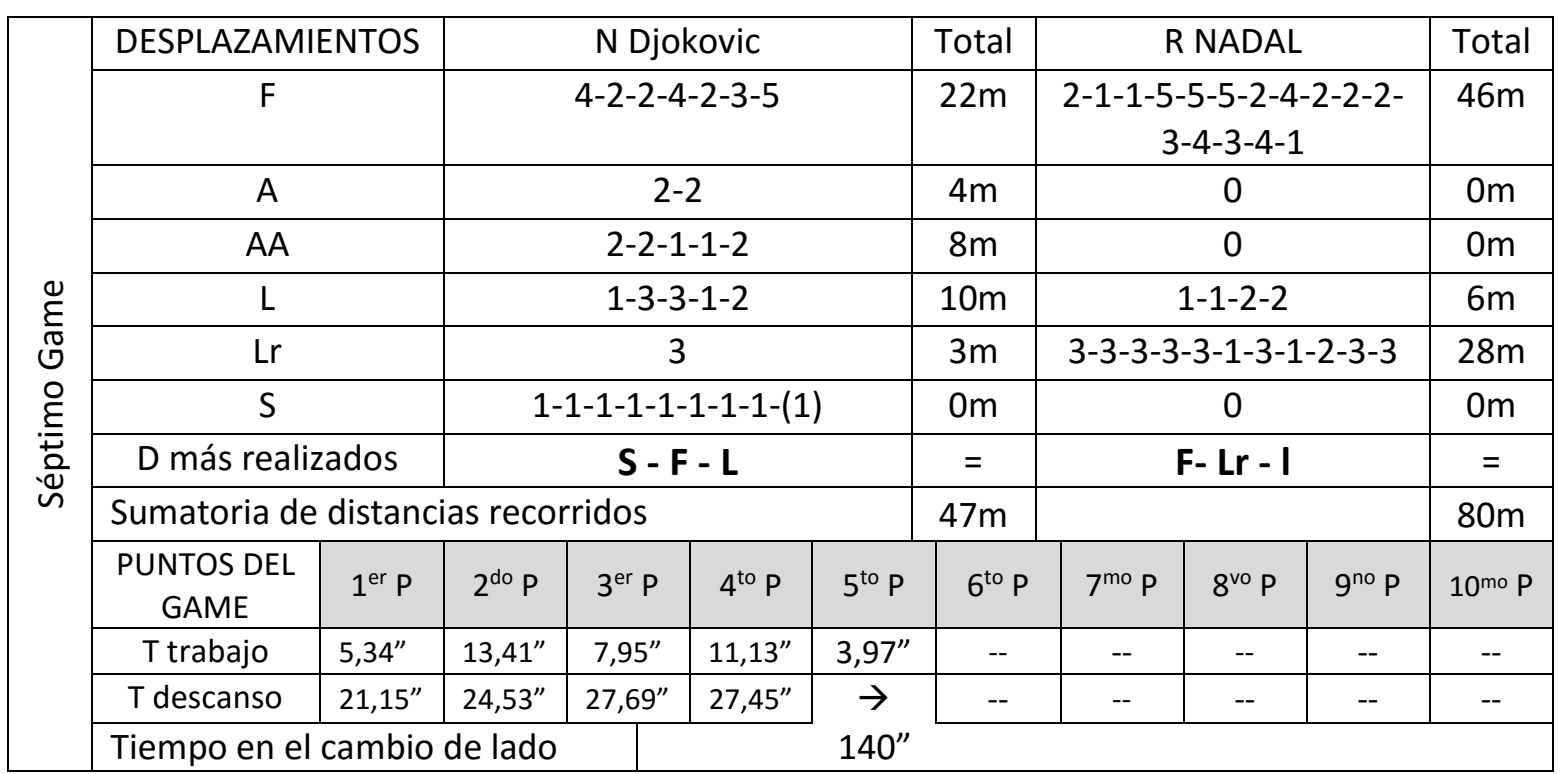

\section{Tabla 68.}

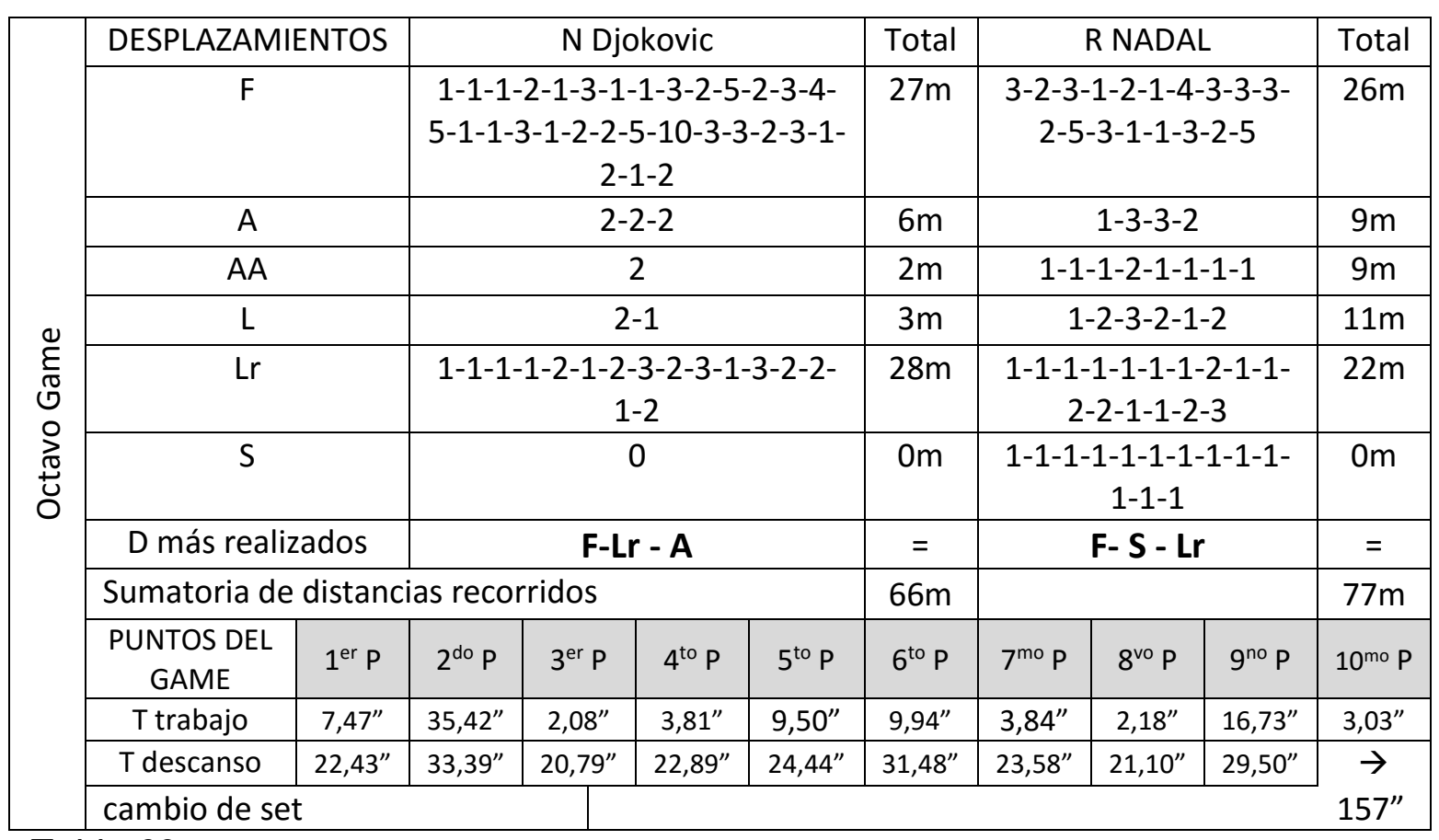

Tabla 69. 


\begin{tabular}{|c|c|c|c|c|c|c|c|c|}
\hline \multicolumn{9}{|c|}{$\begin{array}{l}\text { TIPOS DE DESPLAZAMIENTOS, LOS MÁS UTILIZADOS Y SUS DISTANCIAS. PRIMER SET DEL PARTIDO ENTRE } \\
\text { NOVAK DJOKOVIC Y RAFAEL NADAL, FINAL DE MONTECARLO 2014. SUPERFICIE: POLVO DE LADRILLO. }\end{array}$} \\
\hline $\begin{array}{c}\text { TIPOS DE } \\
\text { DESPLAZAMIENTOS }\end{array}$ & \multicolumn{5}{|c|}{ TOTAL POR SET } & $\begin{array}{l}\text { DISTANCIA MÁS } \\
\text { RECORRIDA }\end{array}$ & $\begin{array}{l}\text { SUMATORIA DE DISTANCIAS } \\
\text { RECORRIDAS POR SET }\end{array}$ & $\begin{array}{c}\text { TOTAL } \\
\text { RECORRIDO }\end{array}$ \\
\hline \multirow{8}{*}{$\begin{array}{l}\text { Desplazamientos de } \\
\text { frente }\end{array}$} & $1 \mathrm{~m}$ & $\begin{array}{r}* * * * * * * * * * * * * * * * \\
* * * * * * * * * * * * \\
\end{array}$ & *** $* * *$ & $* * * * * *$ & & \multirow{8}{*}{2 Metros } & \multirow{6}{*}{$\begin{array}{l}1 \mathrm{~m}=70 \text { veces }=70 \mathrm{~m} \\
2 \mathrm{~m}=95 \text { veces }=190 \mathrm{~m} \\
3 \mathrm{~m}=78 \text { veces }=234 \mathrm{~m} \\
4 \mathrm{~m}=54 \text { veces }=216 \mathrm{~m} \\
5 \mathrm{~m}=25 \text { veces }=125 \mathrm{~m} \\
6 \mathrm{~m}=14 \text { veces }=84 \mathrm{~m} \\
7 \mathrm{~m}=3 \text { veces }=21 \mathrm{~m} \\
8 \mathrm{~m}=1 \text { vez }=8 \mathrm{~m} \\
9 \mathrm{~m}=1 \mathrm{vez}=9 \mathrm{~m} . \\
10 \mathrm{~m}=1 \mathrm{vez}=10 \mathrm{~m} \\
12 \mathrm{~m}=2 \text { veces }=24 \mathrm{~m}\end{array}$} & \multirow{13}{*}{$1.651 \mathrm{~m}}$. \\
\hline & $2 m$ & $\begin{array}{r}* * * * * * * * * * * * * * * * \\
* * * * * * * * * * * * * * * * \\
* * * * * *\end{array}$ & $\begin{array}{l}* * * \\
* * * \\
* * *\end{array}$ & $\begin{array}{l}* * * * * * \\
* * * * * * \\
* *\end{array}$ & $* * * *$ & & & \\
\hline & $3 m$ & $\begin{array}{l}* * * * * * * * * * * * * * * * \\
* * * * * * * * * * * * * * * *\end{array}$ & $* * *$ & $* * * * * *$ & $* * * *$ & & & \\
\hline & $4 m$ & $\begin{array}{r}* * * * * * * * * * * * * * * * \\
* * * *\end{array}$ & $* *$ & $*$ & $* * *$ & & & \\
\hline & $5 \mathrm{~m}$ & $* * * * * * * * *$ & $* *$ & $* * * * * *$ & & & & \\
\hline & $6 \mathrm{~m}$ & $* * * *$ & & & & & & \\
\hline & $7 m$ & $* * *$ & 8 & & & & \multirow{2}{*}{$991 \mathrm{~m}}$. & \\
\hline & $9 m$ & $10 \mathrm{~m}$ & $*$ & $12 \mathrm{~m}$ & $* *$ & & & \\
\hline \multirow{3}{*}{$\begin{array}{l}\text { Desplazamientos hacia } \\
\text { atrás de ejecución }\end{array}$} & $1 \mathrm{~m}$ & & $* *$ & & & \multirow{3}{*}{2 Metros } & \multirow{2}{*}{$\begin{array}{l}1 \mathrm{~m}=9 \text { veces }=9 \mathrm{~m} \\
2 \mathrm{~m}=27 \text { veces }=54 \mathrm{~m} \\
3 \mathrm{~m}=12 \text { veces }=36 \mathrm{~m}\end{array}$} & \\
\hline & $2 m$ & \multicolumn{4}{|c|}{$* * * * * * * * * * * * * * * * * * * * * * * *$} & & & \\
\hline & $3 m$ & \multicolumn{4}{|c|}{$* * * * * * * * * * * *$} & & $99 \mathrm{~m}$. & \\
\hline \multirow{2}{*}{$\begin{array}{l}\text { Desplazamientos hacia } \\
\text { atrás de recuperación }\end{array}$} & $1 m$ & $\begin{array}{r}* * * * * * * * * * * * * * * * \\
* * *\end{array}$ & & $* * * *$ & & \multirow[t]{2}{*}{1 Metro } & \multirow{2}{*}{$\begin{array}{l}1 \mathrm{~m}=53 \text { veces }=53 \mathrm{~m} \\
2 \mathrm{~m}=11 \text { veces }=22 \mathrm{~m}\end{array}$} & \\
\hline & $2 m$ & $* * *$ & $*$ & & & & & \\
\hline
\end{tabular}

Tabla 70. 


\begin{tabular}{|c|c|c|c|c|c|}
\hline \multicolumn{6}{|c|}{$\begin{array}{l}\text { TIPOS DE DESPLAZAMIENTOS, LOS MÁS UTILIZADOS Y SUS DISTANCIAS. PRIMER SET DEL PARTIDO ENTRE } \\
\text { NOVAK DJOKOVIC Y RAFAEL NADAL, FINAL DE MONTECARLO 2014. SUPERFICIE: POLVO DE LADRILLO. }\end{array}$} \\
\hline $\begin{array}{c}\text { TIPOS DE } \\
\text { DESPLAZAMIENTOS }\end{array}$ & \multicolumn{2}{|r|}{ TOTAL POR SET } & $\begin{array}{l}\text { DISTANCIA MÁS } \\
\text { RECORRIDA }\end{array}$ & $\begin{array}{l}\text { SUMATORIA DE DISTANCIAS } \\
\text { RECORRIDAS POR SET }\end{array}$ & $\begin{array}{c}\text { TOTAL } \\
\text { RECORRIDO }\end{array}$ \\
\hline \multirow{3}{*}{$\begin{array}{l}\text { Desplazamientos } \\
\text { laterales de ejecución }\end{array}$} & $1 \mathrm{~m}$ & $* * * * * * * * * * * * * * * * * * * * * * * * * *$ & \multirow{3}{*}{1 Metro } & \multirow{2}{*}{$\begin{array}{l}1 \mathrm{~m}=26 \text { veces }=26 \mathrm{~m} \\
2 \mathrm{~m}=17 \text { veces }=34 \mathrm{~m} \\
3 \mathrm{~m}=4 \text { veces }=12 \mathrm{~m}\end{array}$} & \multirow{7}{*}{$\uparrow$} \\
\hline & $2 m$ & $* * * * * * * * * * * * * * * * * *$ & & & \\
\hline & $3 m$ & $* * * *$ & & $72 m$. & \\
\hline \multirow{3}{*}{$\begin{array}{l}\text { Desplazamientos } \\
\text { laterales de } \\
\text { recuperación. }\end{array}$} & $1 \mathrm{~m}$ & $\begin{array}{c}* * * * * * * * * * * * * * * * * * * * * * * * * * * * * * * * * * * * * * \\
* * * * * * * * * * * * * * * * * * * * * * * * * * * * * * * * *\end{array}$ & \multirow{3}{*}{2 Metros } & \multirow{2}{*}{$\begin{array}{l}1 \mathrm{~m}=69 \text { veces }=69 \mathrm{~m} \\
2 \mathrm{~m}=87 \text { veces }=174 \mathrm{~m} \\
3 \mathrm{~m}=57 \text { veces }=171 \mathrm{~m} .\end{array}$} & \\
\hline & $2 m$ & $\begin{array}{r}* * * * * * * * * * * * * * * * * * * * * * * * * * * * * * * * * * * * \\
* * * * * * * * * * * * * * * * * * * * * * * * * * * * * * * * * * * * \\
\end{array}$ & & & \\
\hline & $3 m$ & $\begin{array}{l}* * * * * * * * * * * * * * * * * * * * * * * * * * * * * * * * * * * * \\
* * * * * * * * * * * * * * * * * * * * *\end{array}$ & & $414 \mathrm{~m}$. & \\
\hline $\begin{array}{l}\text { Desplazamientos } \\
\text { hacia arriba o saltos }\end{array}$ & \multicolumn{2}{|c|}{$\begin{array}{c}* * * * * * * * * * * * * * * * * * * * * * * * * * * * * * * * * * * * * * * * * \\
* * * * * * * * * * * * * * * * * * * * * * * * * * * * * * * * * * * * * * * * * \\
* * *\end{array}$} & \multicolumn{2}{|c|}{ Saltos en servicio $=85$ veces } & \\
\hline
\end{tabular}

Tabla 71.

\begin{tabular}{|c|c|c|c|c|c|c|}
\hline $\begin{array}{c}\text { PORCENTAJES DE USO DE LOS DIFERENTES TIPOS DE DESPLAZAMIENTOS EN EL PRIMER SET DEL PARTIDO ENTRE } \\
\text { NOVAK DJOKOVIC Y RAFAEL NADAL, FINAL DE MONTECARLO 2014. SUPERFICIE: POLVO DE LADRILLO. }\end{array}$ \\
\hline \\
\begin{tabular}{c|c|c|c|c|c|} 
D HACIA \\
ADELANTE
\end{tabular} & $\begin{array}{c}\text { D HACIA ATRÁS } \\
\text { DE EJECUCIÓN }\end{array}$ & $\begin{array}{c}\text { D HACIA ATRÁS DE } \\
\text { RECUPERACIÓN }\end{array}$ & $\begin{array}{c}\text { D LATERALES } \\
\text { DE EJECUCIÓN }\end{array}$ & $\begin{array}{c}\text { D LATERALES DE } \\
\text { RECUPERACIÓN }\end{array}$ & $\begin{array}{c}\text { D HACIA } \\
\text { ARRIBA }\end{array}$ \\
\hline NÚMERO DE VECES & $42,94 \%$ & $5,99 \%$ & $7,99 \%$ & $5,86 \%$ & $26,59 \%$ & $10,61 \%$ \\
\hline DISTANCIA & $60,02 \%$ & $5,99 \%$ & $4,54 \%$ & $3,36 \%$ & $25,07 \%$ & \\
\hline
\end{tabular}

Tabla 72. 


\begin{tabular}{|c|c|c|c|c|}
\hline \multicolumn{5}{|c|}{$\begin{array}{c}\text { TIEMPOS GLOBALES DE LOS MOMENTOS DE TRABAJO Y PAUSA DEL PRIMER SET DEL PARTIDO ENTRE } \\
\text { NOVAK DJOKOVIC Y RAFAEL NADAL, FINAL DE MONTECARLO } 2014\end{array}$} \\
\hline TIEMPOS & \multicolumn{2}{|c|}{$\begin{array}{c}\text { SUMATORIA DE } \\
\text { TIEMPOS POR GAME }\end{array}$} & $\begin{array}{l}\text { SUMATORIA DE TIEMPOS } \\
\text { POR SET }\end{array}$ & $\begin{array}{l}\text { RELACIÓN TIEMPO } \\
\text { DE TRABAJO-PAUSA }\end{array}$ \\
\hline \multirow{8}{*}{ TIEMPO DE TRABAJO } & $1^{\text {er }}$ Game & $37,53^{\prime \prime}$ & \multirow{8}{*}{$\begin{array}{c}604,54 " \text { tiempo real de } \\
\text { trabajo }\end{array}$} & \multirow{16}{*}{$\begin{array}{c}\text { 1" de Trabajo. } \\
\text { x } \\
\text { 3,52" de Pausa. }\end{array}$} \\
\hline & $2^{\text {do }}$ Game & $147,46^{\prime \prime}$ & & \\
\hline & $3^{\text {er }}$ Game & $89,72^{\prime \prime}$ & & \\
\hline & $4^{\text {to }}$ Game & $65,8^{\prime \prime}$ & & \\
\hline & $5^{\text {to }}$ Game & $21,43^{\prime \prime}$ & & \\
\hline & $6^{\text {to }}$ Game & $106,8^{\prime \prime}$ & & \\
\hline & $7^{\text {to }}$ Game & $41,8^{\prime \prime}$ & & \\
\hline & $8^{\text {vo }}$ Game & 94" & & \\
\hline \multirow{8}{*}{ TIEMPO DE DESCANSO } & $1^{\text {er }}$ Game & $164^{\prime \prime}$ & \multirow{8}{*}{$\begin{array}{c}2.132,26 " \text { tiempo real de } \\
\text { descanso }\end{array}$} & \\
\hline & $2^{\text {do }}$ Game & $306,04^{\prime \prime}$ & & \\
\hline & $3^{\text {er }}$ Game & $303,76^{\prime \prime}$ & & \\
\hline & $4^{\text {to }}$ Game & $166,97 "$ & & \\
\hline & $5^{\text {to }}$ Game & $219,72^{\prime \prime}$ & & \\
\hline & $6^{\text {to }}$ Game & $344,35^{\prime \prime}$ & & \\
\hline & $7^{\text {to }}$ Game & $240,82^{\prime \prime}$ & & \\
\hline & $8^{\text {vo }}$ Game & $386,6^{\prime \prime}$ & & \\
\hline
\end{tabular}

Tabla 73.

\begin{tabular}{|c|c|c|c|}
\hline \multicolumn{4}{|c|}{$\begin{array}{l}\text { PROMEDIO DE LOS MOMENTOS ESPECÍFICOS DE TRABAJO Y PAUSA DEL PRIMER SET DEL PARTIDO } \\
\text { ENTRE NOVAK DJOKOVIC Y RAFAEL NADAL, FINAL DE MONTECARLO } 2014\end{array}$} \\
\hline \multicolumn{2}{|c|}{ PROMEDIOS DE TRABAJO } & \multicolumn{2}{|c|}{ PROMEDIOS DE PAUSA } \\
\hline $1^{\text {er }}$ Game & $7,5^{\prime \prime}$ & $1^{\text {er }}$ Game & $21,75^{\prime \prime}$ \\
\hline $2^{\text {do }}$ Game & $12,28^{\prime \prime}$ & $2^{\text {do }}$ Game & $25,08^{\prime \prime}$ \\
\hline $3^{\text {er }}$ Game & $11,21^{\prime \prime}$ & $3^{\text {er }}$ Game & $24,10^{\prime \prime}$ \\
\hline $4^{\text {to }}$ Game & $10,96^{\prime \prime}$ & $4^{\text {to }}$ Game & $25,98^{\prime \prime}$ \\
\hline $5^{\text {to }}$ Game & $4,28^{\prime \prime}$ & $5^{\text {to }}$ Game & $22,18^{\prime \prime}$ \\
\hline $6^{\text {to }}$ Game & $7,62^{\prime \prime}$ & $6^{\text {to }}$ Game & $23,37^{\prime \prime}$ \\
\hline $7^{\mathrm{mo}}$ Game & $8,36^{\prime \prime}$ & $7^{\mathrm{mo}}$ Game & $25,20^{\prime \prime}$ \\
\hline $8^{\text {vo }}$ Game & $9,4^{\prime \prime}$ & $8^{\text {vo }}$ Game & $25,51^{\prime \prime}$ \\
\hline \multicolumn{2}{|c|}{ Promedio total } & \multicolumn{2}{|c|}{ Promedio total } \\
\hline \multicolumn{2}{|c|}{$8,95^{\prime \prime}$} & \multicolumn{2}{|c|}{$24,14 "$} \\
\hline \multicolumn{4}{|c|}{1 1" de trabajo por $2,69 "$ de descanso } \\
\hline
\end{tabular}

Tabla 74. 
5. Análisis punto a punto del primer set del partido entre Novak Djokovic (Serb) y Roger Federer (Sui) en la final del Grand Slam Wimbledon 2014.Y en el que el Serbio $\mathbf{N}$ Djokovic le gana a $\mathbf{R}$ Federer 6-7(5-7), 6-4, 7-6 (7-4), 5-7 y 6-4. Tipo de pista o Cancha (hierba). clasificación ITF categoría 5 (velocidad alta). Ver tablas 76 a 93.

- Análisis de los tiempos y tipos de golpes ejecutados dentro de la cancha antes de comenzar el partido (peloteo previo al partido):

Los jugadores entran a la cancha y después de los actos protocolarios; comienzan a pelotear desde el fondo de la cancha a intensidad media hasta el minuto 1,25 '. Luego se sitúa R Federer cerca a la red para hacer volea 30 " y luego 10" de smash sobre la "T", mientras que Djokovic se queda en el fondo peloteando. Luego se intercambian lo roles y $\mathrm{N}$ Djokovic se sitúa cerca a la red para volear 30" y hacer 10 segundos de Smash sobre la "T". Después de estos, ambos deportistas se sitúan en la línea de fondo de la cancha y realizan 2,38'de servicios, para luego ir a descansar 1' antes de comenzar el partido. En total, los jugadores se tomaron 6,40' desde que empezaron a pelotear para entrar en ritmo de juego. Ver tabla 75.

\begin{tabular}{|c|c|c|c|c|}
\hline \multicolumn{5}{|c|}{$\begin{array}{c}\text { ANÁLISIS DEL USO DE LOS TIPOS DE GOLPES EN EL MOMENTO PREVIO AL } \\
\text { COMIENZO DEL PARTIDO(PELOTEO PREVIO AL PARTIDO) }\end{array}$} \\
\hline Tipo de golpe & N Djokovic & R Federer & Tiempo promedio & $\%$ \\
\hline $\begin{array}{l}\text { (derecha y revés) Peloteo } \\
\text { de fondo de cancha }\end{array}$ & $125 "$ & $\begin{array}{l}85 "+30 "+10 " \\
=125 "\end{array}$ & $125 "$ & $38,69 \%$ \\
\hline Volea cerca a la red & $30 "$ & $30 "$ & $30 "$ & $9,28 \%$ \\
\hline Smash sobre la "T" & $10^{\prime \prime}$ & $10 "$ & $10 "$ & $3,09 \%$ \\
\hline Servicio o saque & 158 & 158 & $158 "$ & $48,91 \%$ \\
\hline
\end{tabular}

Tabla 75. 


\begin{tabular}{|c|c|c|c|c|c|c|c|c|c|c|c|}
\hline \multirow{13}{*}{ 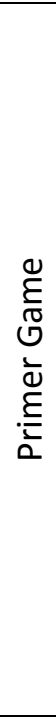 } & \multicolumn{2}{|c|}{ DESPLAZAMIENTOS } & \multicolumn{4}{|c|}{ N Djokovic } & Total & \multicolumn{3}{|c|}{ R FEDERER } & Total \\
\hline & \multicolumn{2}{|c|}{$\mathrm{F}$} & \multicolumn{4}{|c|}{$2-6-2-8-3$} & $21 \mathrm{~m}$ & \multicolumn{3}{|c|}{$4-10-6-3-3-3$} & $29 m$ \\
\hline & \multicolumn{2}{|l|}{ A } & \multicolumn{4}{|c|}{$2-2-2$} & $6 m$ & \multicolumn{3}{|c|}{1} & $1 \mathrm{~m}$ \\
\hline & \multicolumn{2}{|l|}{ AA } & \multicolumn{4}{|c|}{ 1-1-1 } & $3 m$ & \multicolumn{3}{|c|}{0} & Om \\
\hline & \multicolumn{2}{|l|}{$\mathrm{L}$} & \multicolumn{4}{|c|}{$3-2-1-1$} & $7 m$ & \multicolumn{3}{|c|}{$1-1-1-1-1-3-2$} & $10 \mathrm{~m}$ \\
\hline & \multicolumn{2}{|l|}{$\mathrm{Lr}$} & \multicolumn{4}{|c|}{$1-2$} & $3 m$ & \multicolumn{3}{|c|}{$3-1-3-3-2$} & $12 \mathrm{~m}$ \\
\hline & \multicolumn{2}{|l|}{$S$} & \multicolumn{4}{|c|}{ 1-1-1-1-1-1-1 } & $0 m$ & \multicolumn{3}{|c|}{0} & $0 m$ \\
\hline & \multicolumn{2}{|c|}{ D más realizados } & \multicolumn{4}{|c|}{ S-F - L } & $=$ & \multicolumn{3}{|c|}{$L-F-L r$} & $=$ \\
\hline & \multicolumn{6}{|c|}{ Sumatoria de distancias recorridos } & $40 m$ & & & & $52 m$ \\
\hline & $\begin{array}{c}\text { PUNTOS DEL } \\
\text { GAME }\end{array}$ & $1^{\text {er }} P$ & $2^{\text {do }} \mathrm{P}$ & $3^{\text {er }} \mathrm{P}$ & $4^{\text {to }} \mathrm{P}$ & $5^{\text {to }} \mathrm{P}$ & $6^{\text {to }} P$ & $7^{\mathrm{mo}} \mathrm{P}$ & $8^{\text {vo }} \mathrm{P}$ & $9^{\text {no }} P$ & $10^{\text {mo }} \mathrm{P}$ \\
\hline & T trabajo & $8,51^{\prime \prime}$ & $8,22^{\prime \prime}$ & $8^{\prime \prime}$ & $5,06^{\prime \prime}$ & $3,86^{\prime \prime}$ & -- & -- & -- & -- & -- \\
\hline & T descanso & $16,81^{\prime \prime}$ & $16,18^{\prime \prime}$ & $23,42^{\prime \prime}$ & $16,75^{\prime \prime}$ & $\rightarrow$ & -- & -- & -- & -- & -- \\
\hline & \multicolumn{6}{|c|}{\begin{tabular}{|l|l} 
Tiempo en el cambio de lado & $41,41^{\prime \prime}$
\end{tabular}} & & & & & \\
\hline
\end{tabular}

Tabla 76.

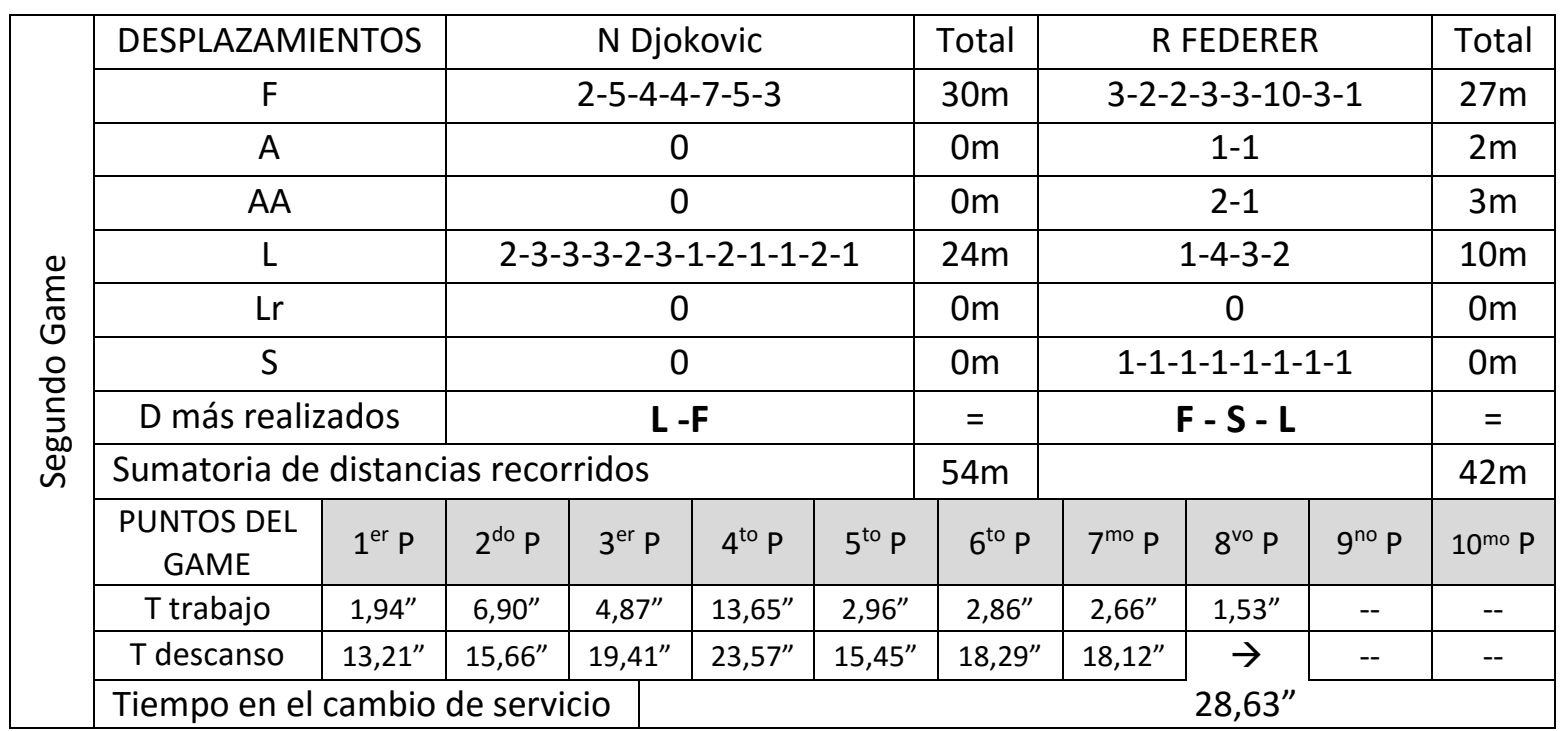

Tabla77. 


\begin{tabular}{|c|c|c|c|c|c|c|c|c|c|c|c|}
\hline & \multicolumn{2}{|c|}{ DESPLAZAMIENTOS } & \multicolumn{4}{|c|}{ N Djokovic } & Total & \multicolumn{3}{|c|}{ R FEDERER } & Total \\
\hline & \multicolumn{2}{|l|}{$\mathrm{F}$} & \multicolumn{4}{|c|}{$2-2-2$} & $6 \mathrm{~m}$ & \multicolumn{3}{|c|}{$1-1-4$} & $6 m$ \\
\hline & \multicolumn{2}{|l|}{ A } & \multicolumn{4}{|c|}{$1-1$} & $2 m$ & \multicolumn{3}{|c|}{0} & $0 \mathrm{~m}$ \\
\hline & \multicolumn{2}{|l|}{ AA } & \multicolumn{4}{|c|}{$1-1$} & $2 m$ & \multicolumn{3}{|c|}{0} & $0 m$ \\
\hline & \multicolumn{2}{|l|}{$\mathrm{L}$} & \multicolumn{4}{|c|}{2} & $2 m$ & \multicolumn{3}{|c|}{$2-3-2-2-3-1$} & $13 m$ \\
\hline हू & \multicolumn{2}{|l|}{ Lr } & \multicolumn{4}{|c|}{$1-2$} & $3 m$ & \multicolumn{3}{|c|}{$3-1$} & $4 m$ \\
\hline$\underbrace{0}_{0}$ & \multicolumn{2}{|l|}{$\mathrm{S}$} & \multicolumn{4}{|c|}{ 1-1-1-1-1-1-1 } & $0 m$ & \multicolumn{3}{|c|}{0} & $0 m$ \\
\hline$\stackrel{ \pm}{0}$ & \multicolumn{2}{|c|}{ D más realizados } & \multicolumn{4}{|c|}{$S-L-A-A A$} & $=$ & \multicolumn{3}{|c|}{$L-F-L r$} & $=$ \\
\hline & \multicolumn{6}{|c|}{ Sumatoria de distancias recorridos } & $15 \mathrm{~m}$ & & & & $23 m$ \\
\hline & $\begin{array}{l}\text { PUNTOS DEL } \\
\text { GAME }\end{array}$ & $1^{\text {er }} \mathrm{P}$ & $2^{\text {do }} \mathrm{P}$ & $3^{\text {er }} \mathrm{P}$ & $4^{\text {to }} \mathrm{P}$ & $5^{\text {to }} \mathrm{P}$ & $6^{\text {to }} \mathrm{P}$ & $7^{\mathrm{mo}} \mathrm{P}$ & $8^{\text {vo }} \mathrm{P}$ & $9^{\text {no }} P$ & $10^{\text {mo }} \mathrm{P}$ \\
\hline & $\mathrm{T}$ trabajo & $2,43^{\prime \prime}$ & $2,90^{\prime \prime}$ & $6,84^{\prime \prime}$ & $4,20^{\prime \prime}$ & -- & -- & -- & -- & -- & -- \\
\hline & T descanso & $17,74^{\prime \prime}$ & $16,61^{\prime \prime}$ & $19,54^{\prime \prime}$ & $\rightarrow$ & -- & -- & -- & -- & -- & -- \\
\hline & \multicolumn{4}{|c|}{ Tiempo en el cambio de lado } & 90" & & & & & & \\
\hline
\end{tabular}

\section{Tabla 78.}

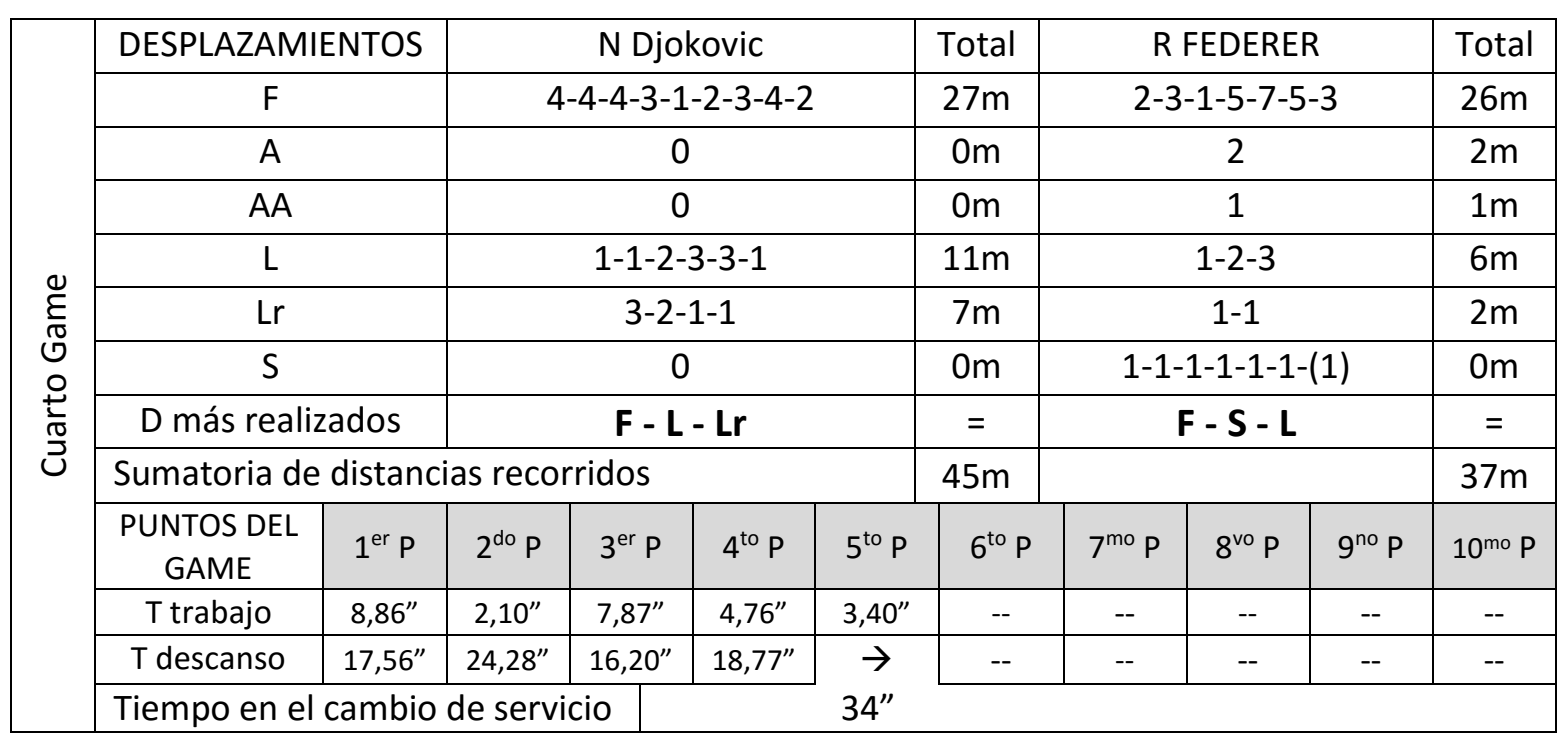

Tabla 79. 


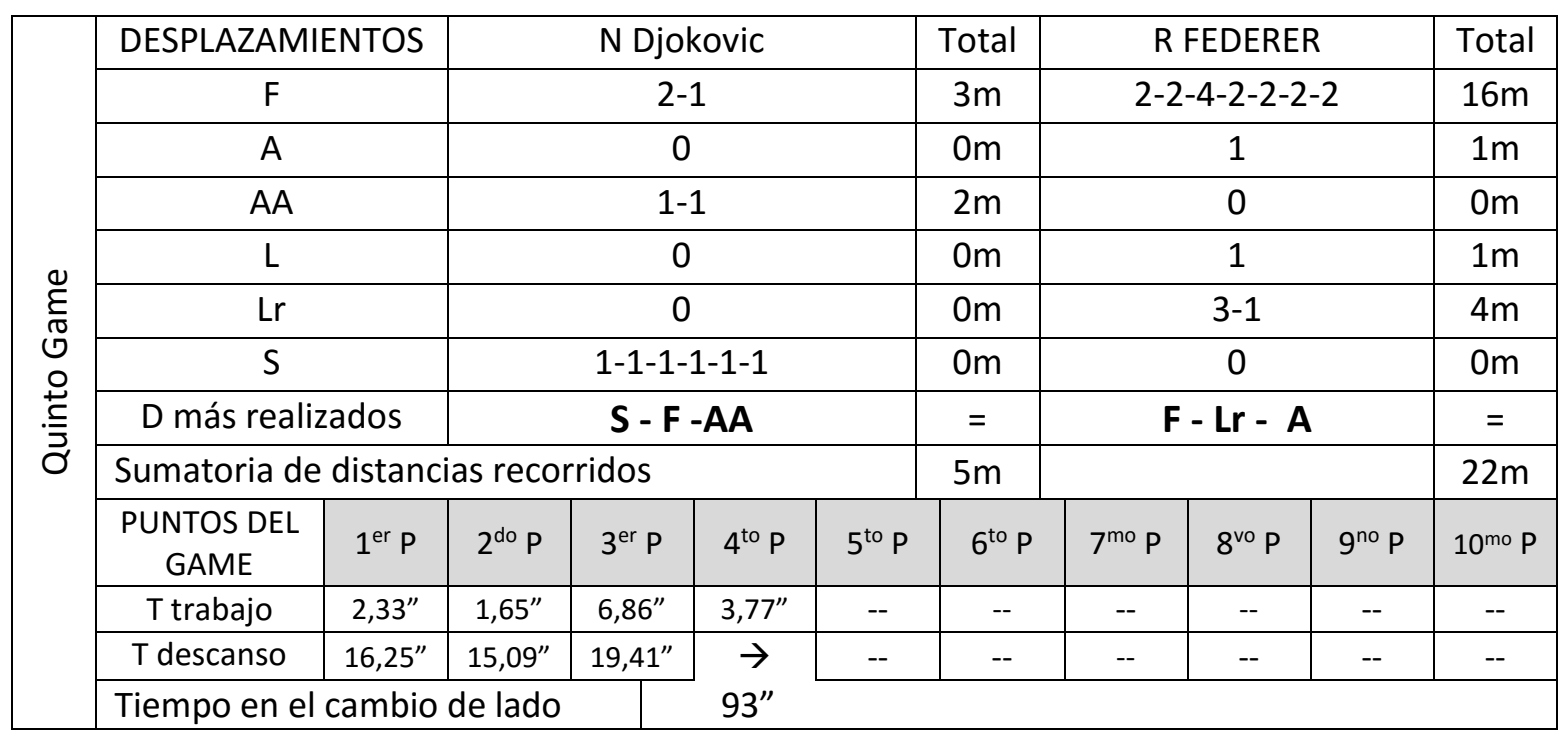

\section{Tabla 80.}

\begin{tabular}{|c|c|c|c|c|c|c|c|c|c|c|c|}
\hline \multirow{13}{*}{ 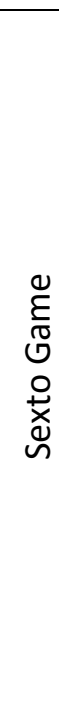 } & \multicolumn{2}{|c|}{ DESPLAZAMIENTOS } & \multicolumn{4}{|c|}{ N Djokovic } & Total & \multicolumn{3}{|c|}{ R FEDERER } & Total \\
\hline & \multicolumn{2}{|l|}{$\mathrm{F}$} & \multicolumn{4}{|c|}{$2-3-2-3-3-1-3$} & $17 m$ & \multicolumn{3}{|c|}{$4-5-5$} & $14 \mathrm{~m}$ \\
\hline & \multicolumn{2}{|l|}{ A } & \multicolumn{4}{|c|}{0} & $0 \mathrm{~m}$ & \multicolumn{3}{|c|}{0} & $0 m$ \\
\hline & \multicolumn{2}{|l|}{$\mathrm{AA}$} & \multicolumn{4}{|c|}{0} & $0 \mathrm{~m}$ & \multicolumn{3}{|c|}{0} & $0 m$ \\
\hline & \multicolumn{2}{|l|}{$\mathrm{L}$} & \multicolumn{4}{|c|}{0} & $0 \mathrm{~m}$ & \multicolumn{3}{|c|}{0} & $0 m$ \\
\hline & \multicolumn{2}{|l|}{$\mathrm{Lr}$} & \multicolumn{4}{|c|}{2} & $2 m$ & \multicolumn{3}{|c|}{0} & $0 m$ \\
\hline & \multicolumn{2}{|l|}{$S$} & \multicolumn{4}{|c|}{0} & $0 m$ & \multicolumn{3}{|c|}{ 1-1-1-1-1-1-1-1 } & $0 m$ \\
\hline & \multicolumn{2}{|c|}{ D más realizados } & \multicolumn{4}{|c|}{ F - Lr } & $=$ & \multicolumn{3}{|c|}{$S-F$} & $=$ \\
\hline & \multicolumn{6}{|c|}{ Sumatoria de distancias recorridos } & $19 m$ & & & & $14 \mathrm{~m}$ \\
\hline & $\begin{array}{l}\text { PUNTOS DEL } \\
\text { GAME }\end{array}$ & $1^{\text {er }} \mathrm{P}$ & $2^{\text {do }} \mathrm{P}$ & $3^{\text {er } P}$ & $4^{\text {to }} \mathrm{P}$ & $5^{\text {to }} P$ & $6^{\text {to }} p$ & $7^{\text {mo }} \mathrm{p}$ & $8^{\text {vo }} \mathrm{P}$ & $9^{\text {no }} P$ & $10^{\text {mo }} \mathrm{P}$ \\
\hline & T trabajo & $2,04^{\prime \prime}$ & $2,44^{\prime \prime}$ & $1,96^{\prime \prime}$ & $1,72^{\prime \prime}$ & $1,69^{\prime \prime}$ & $2,41^{\prime \prime}$ & -- & -- & -- & -- \\
\hline & T descanso & $12,46^{\prime \prime}$ & $13,43^{\prime \prime}$ & $16,32^{\prime \prime}$ & $16,07 "$ & $15,21^{\prime \prime}$ & $\rightarrow$ & -- & -- & -- & -- \\
\hline & \multicolumn{6}{|c|}{ Tiempo en el cambio de servicio } & \multicolumn{5}{|l|}{$30,14^{\prime \prime}$} \\
\hline
\end{tabular}

\section{Tabla 81.}




\begin{tabular}{|c|c|c|c|c|c|c|c|c|c|c|c|}
\hline \multirow{13}{*}{ 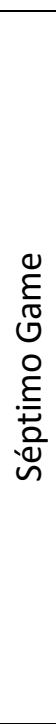 } & \multicolumn{2}{|c|}{ DESPLAZAMIENTOS } & \multicolumn{4}{|c|}{ N Djokovic } & Total & \multicolumn{3}{|c|}{ R FEDERER } & Total \\
\hline & \multicolumn{2}{|l|}{$\mathrm{F}$} & \multicolumn{4}{|c|}{$3-3-2$} & $8 m$ & \multicolumn{3}{|c|}{$1-2-5-3-2$} & $13 m$ \\
\hline & \multicolumn{2}{|l|}{ A } & \multicolumn{4}{|c|}{0} & $0 m$ & \multicolumn{3}{|c|}{0} & $0 m$ \\
\hline & \multicolumn{2}{|l|}{ AA } & \multicolumn{4}{|c|}{ 1-1-1 } & $3 m$ & \multicolumn{3}{|c|}{1} & $1 \mathrm{~m}$ \\
\hline & \multicolumn{2}{|l|}{$\mathrm{L}$} & \multicolumn{4}{|c|}{0} & $0 m$ & \multicolumn{3}{|c|}{$1-1$} & $2 m$ \\
\hline & \multicolumn{2}{|l|}{$\mathrm{Lr}$} & \multicolumn{4}{|c|}{$1-2$} & $3 m$ & \multicolumn{3}{|c|}{$2-2-3-3$} & $10 \mathrm{~m}$ \\
\hline & \multicolumn{2}{|l|}{$S$} & \multicolumn{4}{|c|}{ 1-1-1-1-1-1-1 } & $0 m$ & \multicolumn{3}{|c|}{0} & $0 m$ \\
\hline & \multicolumn{2}{|c|}{ D más realizados } & \multicolumn{4}{|c|}{$S-F-A A$} & $=$ & \multicolumn{3}{|c|}{$F-L r-L$} & $=$ \\
\hline & \multicolumn{6}{|c|}{ Sumatoria de distancias recorridos } & $14 m$ & & & & $26 m$ \\
\hline & $\begin{array}{c}\text { PUNTOS DEL } \\
\text { GAME }\end{array}$ & $1^{\text {er }} \mathrm{P}$ & $2^{\text {do }} \mathrm{P}$ & $3^{\text {er }} \mathrm{P}$ & $4^{\text {to }} \mathrm{P}$ & $5^{\text {to }} \mathrm{P}$ & $6^{\text {to }} \mathrm{P}$ & $7^{\mathrm{mo}} \mathrm{P}$ & $8^{\text {vo } P}$ & $9^{\text {no }} P$ & $10^{\text {mo }} P$ \\
\hline & T trabajo & $1,36^{\prime \prime}$ & $8,92^{\prime \prime}$ & $1,71^{\prime \prime}$ & $2,50^{\prime \prime}$ & $4,68^{\prime \prime}$ & -- & -- & -- & -- & -- \\
\hline & T descanso & $15,19^{\prime \prime}$ & $19,65^{\prime \prime}$ & $16,52^{\prime \prime}$ & $16,32^{\prime \prime}$ & $\rightarrow$ & -- & -- & -- & -- & -- \\
\hline & \multicolumn{5}{|c|}{ Tiempo en el cambio de lado } & $94^{\prime \prime}$ & & & & & \\
\hline
\end{tabular}

\section{Tabla 82.}

\begin{tabular}{|c|c|c|c|c|c|c|c|c|c|c|c|}
\hline \multirow{13}{*}{ 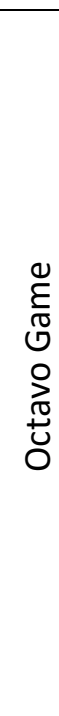 } & \multicolumn{2}{|c|}{ DESPLAZAMIENTOS } & \multicolumn{4}{|c|}{ N Djokovic } & Total & \multicolumn{3}{|c|}{ R FEDERER } & Total \\
\hline & \multicolumn{2}{|l|}{$\mathrm{F}$} & \multicolumn{4}{|c|}{$2-3-2-2-1-2-4-1-2-4-3-4-3$} & $33 m$ & \multicolumn{3}{|c|}{$4-2-4-5-6-2-1-4-5-2$} & $35 \mathrm{~m}$ \\
\hline & \multicolumn{2}{|l|}{ A } & \multicolumn{4}{|c|}{1} & $1 \mathrm{~m}$ & \multicolumn{3}{|c|}{2} & $2 m$ \\
\hline & \multicolumn{2}{|l|}{$\mathrm{AA}$} & \multicolumn{4}{|c|}{1} & $1 \mathrm{~m}$ & \multicolumn{3}{|c|}{0} & $0 m$ \\
\hline & \multicolumn{2}{|l|}{$\mathrm{L}$} & \multicolumn{4}{|c|}{$2-2-2-2$} & $8 m$ & \multicolumn{3}{|c|}{$1-1-1$} & $3 m$ \\
\hline & \multicolumn{2}{|l|}{$\mathrm{Lr}$} & \multicolumn{4}{|c|}{$2-1-1-2-1-3$} & $10 \mathrm{~m}$ & \multicolumn{3}{|c|}{ 1-3-1-1 } & $6 \mathrm{~m}$ \\
\hline & \multicolumn{2}{|l|}{$\mathrm{S}$} & & & & & $0 m$ & \multicolumn{3}{|c|}{ 1-1-1-1-1-1-1-1-1-1 } & $0 m$ \\
\hline & \multicolumn{2}{|c|}{ D más realizados } & \multicolumn{4}{|c|}{$F-L r-L$} & $=$ & \multicolumn{3}{|c|}{$F-S-L r$} & $=$ \\
\hline & \multicolumn{6}{|c|}{ Sumatoria de distancias recorridos } & $52 m$ & & & & $46 \mathrm{~m}$ \\
\hline & $\begin{array}{l}\text { PUNTOS DEL } \\
\text { GAME }\end{array}$ & $1^{\text {er }} \mathrm{P}$ & $2^{\text {do }} \mathrm{p}$ & $3^{\text {er } P}$ & $4^{\text {to }} \mathrm{P}$ & $5^{\text {to }} P$ & $6^{\text {to }} \mathrm{P}$ & $7^{\text {mo }} \mathrm{p}$ & $8^{\text {vo }} \mathrm{P}$ & $9^{\text {no }} P$ & $10^{\text {mo }} \mathrm{P}$ \\
\hline & T trabajo & $3,64^{\prime \prime}$ & $1,33^{\prime \prime}$ & $10,73^{\prime \prime}$ & $2,19^{\prime \prime}$ & $2,73^{\prime \prime}$ & $11,69 "$ & $1,89^{\prime \prime}$ & $3,39^{\prime \prime}$ & -- & -- \\
\hline & T descanso & $14,35^{\prime \prime}$ & $13^{\prime \prime}$ & $20,55^{\prime \prime}$ & $17,30^{\prime \prime}$ & 17,15 & $22,66 "$ & $16,37^{\prime \prime}$ & $\rightarrow$ & -- & -- \\
\hline & \multicolumn{6}{|c|}{ Tiempo en el cambio de servicio } & \multicolumn{5}{|c|}{$36,50^{\prime \prime}$} \\
\hline
\end{tabular}

Tabla 83. 


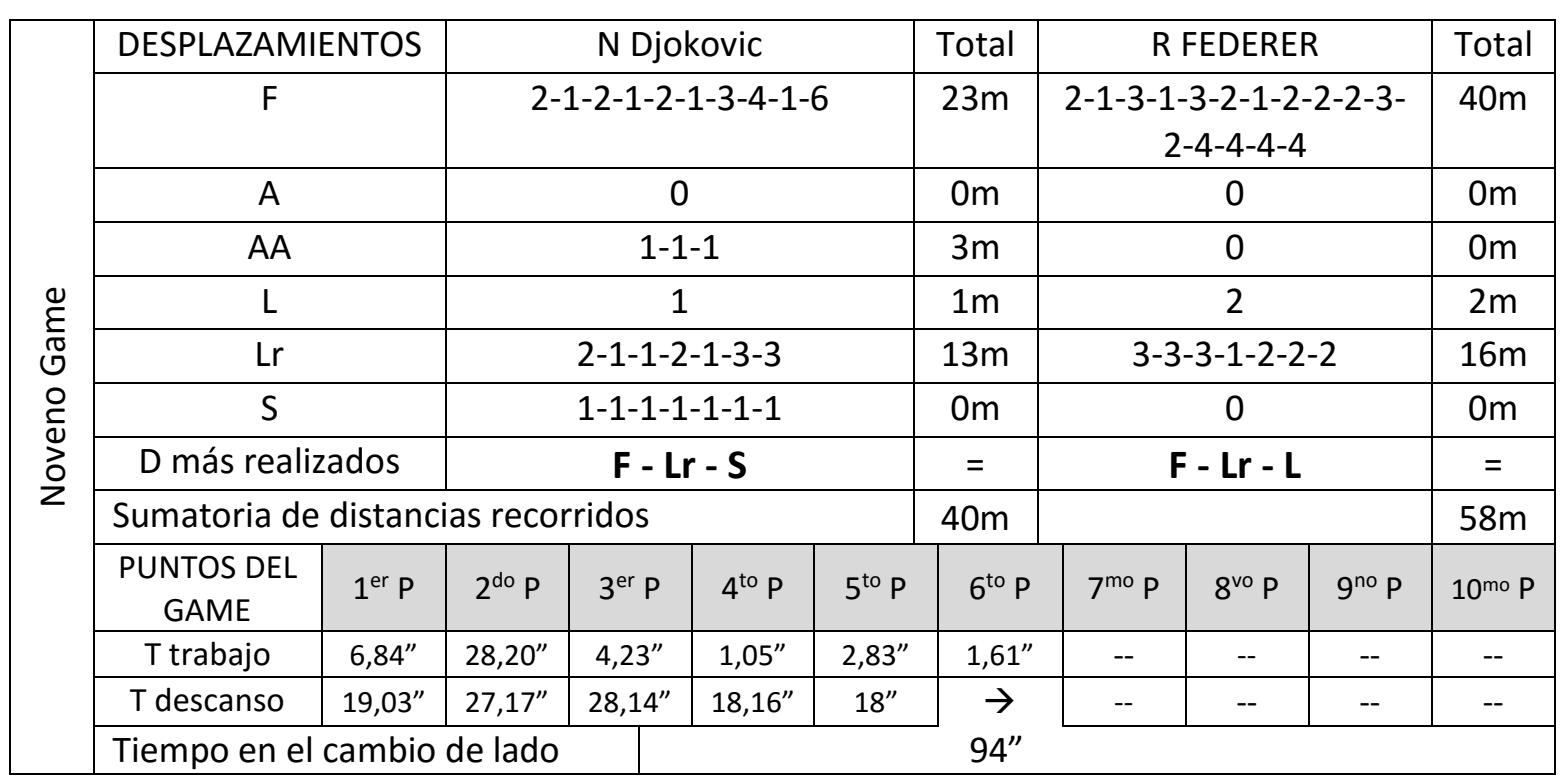

\section{Tabla 84.}

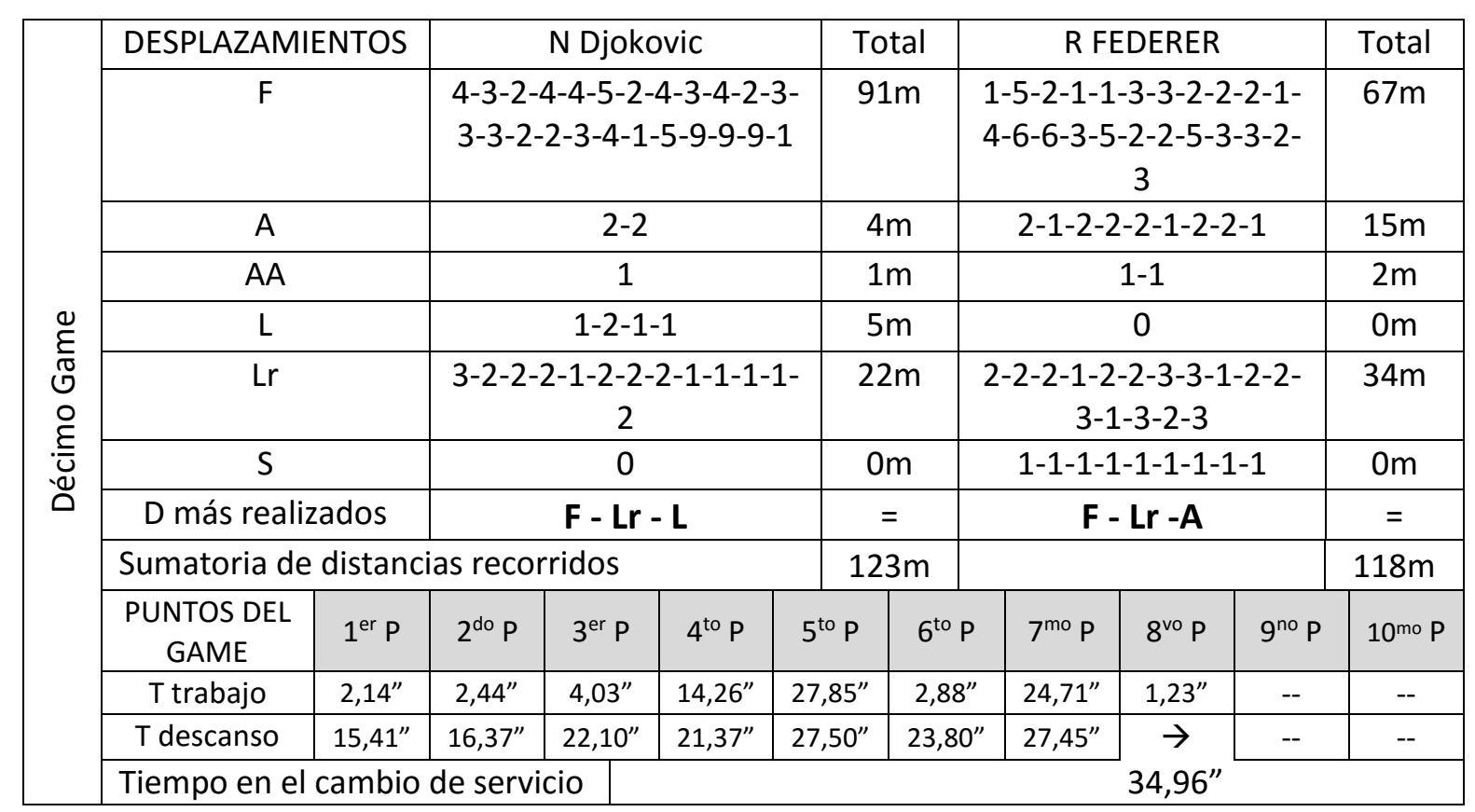

Tabla 85. 


\begin{tabular}{|c|c|c|c|c|c|c|c|c|c|c|c|}
\hline \multirow{13}{*}{ 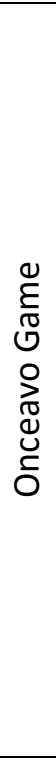 } & \multicolumn{2}{|c|}{ DESPLAZAMIENTOS } & \multicolumn{4}{|c|}{ N Djokovic } & Total & \multicolumn{3}{|c|}{ R FEDERER } & Total \\
\hline & \multicolumn{2}{|l|}{$\mathrm{F}$} & \multicolumn{4}{|c|}{$4-3-2-2-3-4-1-2-1-4-4$} & $30 \mathrm{~m}$ & \multicolumn{3}{|c|}{$\begin{array}{c}1-1-3-5-5-4-1-2-3-2-3- \\
3-3-1-7-8-2-3-4\end{array}$} & $61 \mathrm{~m}$ \\
\hline & \multicolumn{2}{|l|}{ A } & \multicolumn{4}{|c|}{0} & $0 m$ & \multicolumn{3}{|c|}{0} & $0 m$ \\
\hline & \multicolumn{2}{|l|}{ AA } & \multicolumn{4}{|c|}{ 1-1-1-1 } & $4 m$ & \multicolumn{3}{|c|}{0} & $0 m$ \\
\hline & \multicolumn{2}{|l|}{$\mathrm{L}$} & \multicolumn{4}{|c|}{$2-1-1-2-3-1$} & $10 \mathrm{~m}$ & \multicolumn{3}{|c|}{$2-2$} & $4 m$ \\
\hline & \multicolumn{2}{|l|}{$\mathrm{Lr}$} & \multicolumn{4}{|c|}{$2-1-1-3-1-2$} & $10 m$ & \multicolumn{3}{|c|}{$3-4-3-3-3-2-1-2-1-1$} & $23 \mathrm{~m}$ \\
\hline & \multicolumn{2}{|l|}{$S$} & \multicolumn{4}{|c|}{0} & $0 m$ & \multicolumn{3}{|c|}{ 1-1-1-1-1-1-1-1-1 } & $0 m$ \\
\hline & \multicolumn{2}{|c|}{ D más realizados } & \multicolumn{4}{|c|}{$F-L-L r$} & $=$ & \multicolumn{3}{|c|}{$F-L r-S$} & $=$ \\
\hline & \multicolumn{6}{|c|}{ Sumatoria de distancias recorridos } & $54 m$ & & & & $88 \mathrm{~m}$ \\
\hline & $\begin{array}{l}\text { PUNTOS DEL } \\
\text { GAME }\end{array}$ & $1^{\text {er }} P$ & $2^{\text {do }} \mathrm{P}$ & $3^{\text {er } P}$ & $4^{\text {to }} \mathrm{P}$ & $5^{\text {to }} \mathrm{P}$ & $6^{\text {to }} \mathrm{P}$ & $7^{\mathrm{mo}} \mathrm{P}$ & $8^{\text {vo }} \mathrm{P}$ & $9^{\text {no }} P$ & $10^{\text {mo }} \mathrm{P}$ \\
\hline & $\mathrm{T}$ trabajo & $4,87^{\prime \prime}$ & $18,48^{\prime \prime}$ & $1,83^{\prime \prime}$ & $9,25^{\prime \prime}$ & $2,14^{\prime \prime}$ & $1,20^{\prime \prime}$ & $7,47^{\prime \prime}$ & $3,17^{\prime \prime}$ & -- & -- \\
\hline & T descanso & $21,37^{\prime \prime}$ & $27,48^{\prime \prime}$ & $18,56^{\prime \prime}$ & $25,06^{\prime \prime}$ & $16,62^{\prime \prime}$ & $27,46^{\prime \prime}$ & $18,65^{\prime \prime}$ & $\rightarrow$ & - & -- \\
\hline & \multicolumn{6}{|c|}{ Tiempo en el cambio de lado } & \multicolumn{5}{|c|}{ 93" } \\
\hline
\end{tabular}

\section{Tabla 86.}

\begin{tabular}{|c|c|c|c|c|c|c|c|c|c|c|c|}
\hline \multirow{13}{*}{ 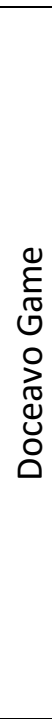 } & \multirow{2}{*}{\multicolumn{2}{|c|}{$\frac{\text { DESPLAZAMIENTOS }}{\mathrm{F}}$}} & \multicolumn{4}{|c|}{ N Djokovic } & Total & \multicolumn{3}{|c|}{ R FEDERER } & Total \\
\hline & & & \multicolumn{4}{|c|}{$4-2-2-2-2-8-3-4-2$} & $29 m$ & \multicolumn{3}{|c|}{$5-3-5-3-4-3-1-1-2$} & $27 m$ \\
\hline & \multicolumn{2}{|l|}{ A } & \multicolumn{4}{|c|}{$2-1-2$} & $5 \mathrm{~m}$ & \multicolumn{3}{|c|}{1} & $1 \mathrm{~m}$ \\
\hline & \multicolumn{2}{|l|}{ AA } & \multicolumn{4}{|c|}{1} & $1 \mathrm{~m}$ & \multicolumn{3}{|c|}{1} & $1 \mathrm{~m}$ \\
\hline & \multicolumn{2}{|l|}{$\mathrm{L}$} & \multicolumn{4}{|c|}{ 3-1 } & $4 m$ & \multicolumn{3}{|c|}{0} & $0 m$ \\
\hline & \multicolumn{2}{|l|}{ Lr } & \multicolumn{4}{|c|}{$1-1-2$} & $4 m$ & \multicolumn{3}{|c|}{$3-1-2$} & $6 m$ \\
\hline & \multicolumn{2}{|l|}{$S$} & \multicolumn{4}{|c|}{0} & $0 \mathrm{~m}$ & \multicolumn{3}{|c|}{ 1-1-1-1-1-1-1-1 } & $0 \mathrm{~m}$ \\
\hline & \multicolumn{2}{|c|}{ D más realizados } & \multicolumn{4}{|c|}{$F-A-L r$} & $=$ & \multicolumn{3}{|c|}{$\mathbf{F}-\mathbf{S}-\mathbf{L r}$} & $=$ \\
\hline & \multicolumn{6}{|c|}{ Sumatoria de distancias recorridos } & $43 \mathrm{~m}$ & & & & $35 \mathrm{~m}$ \\
\hline & $\begin{array}{l}\text { PUNTOS DEL } \\
\text { GAME }\end{array}$ & $1^{\text {er }} \mathrm{P}$ & $2^{\text {do }} \mathrm{P}$ & $3^{\text {er }} \mathrm{P}$ & $4^{\text {to }} \mathrm{P}$ & $5^{\text {to }} \mathrm{P}$ & $6^{\text {to }} \mathrm{P}$ & $7^{\mathrm{mo}} \mathrm{P}$ & $8^{\mathrm{vo}} \mathrm{P}$ & $9^{\text {no }} P$ & $10^{\text {mo }} \mathrm{P}$ \\
\hline & $\mathrm{T}$ trabajo & $1,96^{\prime \prime}$ & $2,06^{\prime \prime}$ & $3,93^{\prime \prime}$ & $5,95^{\prime \prime}$ & $5,69^{\prime \prime}$ & $5,10^{\prime \prime}$ & -- & -- & -- & -- \\
\hline & T descanso & $13,91^{\prime \prime}$ & $13,96^{\prime \prime}$ & $19,11^{\prime \prime}$ & $19,74^{\prime \prime}$ & $16,53^{\prime \prime}$ & $\rightarrow$ & -- & -- & -- & -- \\
\hline & \multicolumn{6}{|c|}{ Tiempo en el cambio de servicio } & $28,62^{\prime}$ & & & & \\
\hline
\end{tabular}

\section{Tabla 87.}




\begin{tabular}{|c|c|c|c|c|c|c|c|c|c|c|c|}
\hline \multirow{16}{*}{ 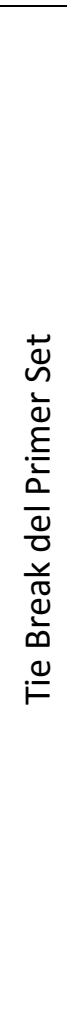 } & \multicolumn{2}{|c|}{ DESPLAZAMIENTOS } & \multicolumn{4}{|c|}{ N Djokovic } & Total & \multicolumn{3}{|c|}{ R FEDERER } & Total \\
\hline & \multicolumn{2}{|l|}{$\mathrm{F}$} & \multicolumn{4}{|c|}{$\begin{array}{c}5-1-1-1-4-2-5-3-7-2-4-1-5- \\
5-6-2-4-1-1-4-4-6-9-2-5\end{array}$} & $92 m$ & \multicolumn{3}{|c|}{$\begin{array}{c}4-4-6-3-1-2-2-2-3-2-8- \\
5-1-3-4-1-2-4-3-2-2-5- \\
4-2-1-5-3-5-6\end{array}$} & $95 m$ \\
\hline & \multicolumn{2}{|l|}{ A } & \multicolumn{4}{|c|}{$2-1-2-1-2-2-1$} & $11 \mathrm{~m}$ & \multicolumn{3}{|c|}{$3-3-3-2-2$} & $14 \mathrm{~m}$ \\
\hline & \multicolumn{2}{|l|}{$A A$} & \multicolumn{4}{|c|}{$1-1$} & $2 m$ & \multicolumn{3}{|c|}{$1-1$} & $2 m$ \\
\hline & \multicolumn{2}{|l|}{$\mathrm{L}$} & \multicolumn{4}{|c|}{$1-2-1-3-1-1-3-3-2-2-2$} & $21 \mathrm{~m}$ & \multicolumn{3}{|c|}{$1-3-1$} & $4 m$ \\
\hline & \multicolumn{2}{|l|}{ Lr } & \multicolumn{4}{|c|}{$2-1-2-2-2-3-2-2-3-2$} & $21 \mathrm{~m}$ & \multicolumn{3}{|c|}{$\begin{array}{c}1-2-1-3-2-2-2-2-2-2-3- \\
2-2-3-3-2-2\end{array}$} & $36 \mathrm{~m}$ \\
\hline & \multicolumn{2}{|l|}{$S$} & \multicolumn{4}{|c|}{ 1-1-1-1-1-1-1 } & $0 m$ & \multicolumn{3}{|c|}{ 1-1-1-1-1-1-1-1-1 } & $0 \mathrm{~m}$ \\
\hline & \multicolumn{2}{|c|}{ D más realizados } & \multicolumn{4}{|c|}{$F-L r-L$} & $=$ & \multicolumn{3}{|c|}{$F-L r-L$} & $=$ \\
\hline & \multicolumn{6}{|c|}{ Sumatoria de distancias recorridas } & $147 m$ & & & & $151 \mathrm{~m}$ \\
\hline & $\begin{array}{l}\text { PUNTOS DEL } \\
\text { TIE BREAK }\end{array}$ & $1^{\text {er }} \mathrm{P}$ & $2^{\text {do }} P$ & $3^{\text {er }} \mathrm{P}$ & $4^{\text {to }} \mathrm{P}$ & $5^{\text {to }} \mathrm{P}$ & $6^{\text {to }} \mathrm{P}$ & $7^{\mathrm{mo}} \mathrm{P}$ & $8^{\text {vo }} \mathrm{P}$ & $9^{\text {no }} P$ & $10^{\text {mo }} \mathrm{P}$ \\
\hline & $\mathrm{T}$ trabajo & $6,29^{\prime \prime}$ & $26,09^{\prime \prime}$ & $1,64^{\prime \prime}$ & $4,24^{\prime \prime}$ & $3,78^{\prime \prime}$ & $7,88^{\prime \prime}$ & $19,82^{\prime \prime}$ & $6^{\prime \prime}$ & $1,04^{\prime \prime}$ & $15,75^{\prime \prime}$ \\
\hline & T descanso & $23,40^{\prime \prime}$ & $22,67^{\prime \prime}$ & $29,40^{\prime \prime}$ & $21,31^{\prime \prime}$ & $27,23^{\prime}$ & \begin{tabular}{l|l|}
$\prime \prime$ & $44,42^{\prime \prime}$
\end{tabular} & $31,79^{\prime \prime}$ & $26,49^{\prime \prime}$ & $25,17^{\prime \prime}$ & $33,95^{\prime \prime}$ \\
\hline & $\begin{array}{l}\text { PUNTOS DEL } \\
\text { TIE BREAK }\end{array}$ & $11^{\text {vo }} \mathrm{P}$ & $12^{\mathrm{vo}} \mathrm{P}$ & $13^{\text {vo }} \mathrm{P}$ & $14^{\mathrm{vo} P}$ & $15^{\text {vo }} \mathrm{p}$ & $16^{\mathrm{vo}} \mathrm{P}$ & $17^{\text {vo }} \mathrm{P}$ & $18^{\text {vo }} \mathrm{P}$ & $19^{v o} \mathrm{P}$ & $20^{\text {vo }} \mathrm{P}$ \\
\hline & T trabajo & $3,10^{\prime \prime}$ & $8,72^{\prime \prime}$ & $2,33^{\prime \prime}$ & $1,05^{\prime \prime}$ & $1,96^{\prime \prime}$ & $5,64^{\prime \prime}$ & -- & -- & -- & -- \\
\hline & T descanso & $34,94^{\prime \prime}$ & $51,40^{\prime \prime}$ & $22,58^{\prime \prime}$ & $18,93^{\prime \prime}$ & $29,08^{\prime}$ & $\rightarrow$ & -- & -- & -- & -- \\
\hline & Tiempo en el & cambio & de set & & & & 176" & & & & \\
\hline
\end{tabular}

Tabla 88. 


\begin{tabular}{|c|c|c|c|c|c|c|}
\hline \multicolumn{7}{|c|}{$\begin{array}{l}\text { TIPOS DE DESPLAZAMIENTOS, LOS MÁS UTILIZADOS Y SUS DISTANCIAS. PRIMER SET DEL PARTIDO DE FINAL DE GRAND SLAM WIMBLEDON } \\
\text { 2014, ENTRE NOVAK DJOKOVIC Y ROGER FEDERER. SUPERFICIE: HIERVA. }\end{array}$} \\
\hline $\begin{array}{l}\text { TIPOS DE } \\
\text { DESPLAZAMIENTOS }\end{array}$ & \multicolumn{3}{|c|}{ TOTAL POR SET } & $\begin{array}{l}\text { DISTANCIA MÁS } \\
\text { RECORRIDA }\end{array}$ & $\begin{array}{l}\text { SUMATORIA DE DISTANCIAS } \\
\text { RECORRIDAS POR SET }\end{array}$ & $\begin{array}{l}\text { TOTAL } \\
\text { RECORRIDO }\end{array}$ \\
\hline \multirow{8}{*}{$\begin{array}{l}\text { Desplazamientos de } \\
\quad \text { frente }\end{array}$} & $1 \mathrm{~m}$ & $\begin{array}{r}* * * * * * * * * * * * * * * * * * * * * * \\
* * * *\end{array}$ & $* * * * * * * *$ & \multirow{8}{*}{ Me } & \multirow{6}{*}{$\begin{array}{l}1 \mathrm{~m}=42 \text { veces }=42 \mathrm{~m} \\
2 \mathrm{~m}=74 \text { veces }=148 \mathrm{~m} \\
3 \mathrm{~m}=57 \text { veces }=171 \mathrm{~m} \\
4 \mathrm{~m}=44 \text { veces }=176 \mathrm{~m} \\
5 \mathrm{~m}=26 \text { veces }=130 \mathrm{~m} \\
6 \mathrm{~m}=15 \text { veces }=90 \mathrm{~m} \\
7 \mathrm{~m}=4 \text { veces }=28 \mathrm{~m} \\
8 \mathrm{~m}=4 \text { veces }=32 \mathrm{~m} \\
9 \mathrm{~m}=4 \text { veces }=36 \mathrm{~m} \\
10 \mathrm{~m}=1 \text { vez }=10 \mathrm{~m}\end{array}$} & \multirow{13}{*}{$1.392 \mathrm{~m}$} \\
\hline & $2 m$ & $\begin{array}{r}* * * * * * * * * * * * * * * * * * * * * * \\
* * * * * * * * * * * * * * * * * *)\end{array}$ & $\begin{array}{l}* * * * * * * * \\
* * * * * * *\end{array}$ & & & \\
\hline & $3 m$ & $\begin{array}{r}* * * * * * * * * * * * * * * * * * * * * * * \\
* * * * * * * * * * * * *\end{array}$ & $* * * * * * * *$ & & & \\
\hline & $4 m$ & $\begin{array}{r}* * * * * * * * * * * * * * * * * * * * * * \\
* * * * * *\end{array}$ & $* * * * * * * *$ & & & \\
\hline & $5 m$ & $* * * * * * * * * * * * * * * * *$ & & & & \\
\hline & $6 \mathrm{~m}$ & $* * * * * * * * * * *$ & & & & \\
\hline & $7 \mathrm{~m}$ & $* * * *$ & $* * * *$ & & \multirow{2}{*}{$863 \mathrm{~m}}$. & \\
\hline & $9 m$ & $* * * *$ & $* *$ & & & \\
\hline \multirow{3}{*}{$\begin{array}{l}\text { Desplazamientos } \\
\text { hacia atrás de } \\
\text { ejecución }\end{array}$} & $1 \mathrm{~m}$ & $* * * * * * * * * * *$ & & \multirow{3}{*}{2 Metros } & $1 \mathrm{~m}=15$ veces $=15 \mathrm{~m}$ & \\
\hline & $2 m$ & $* * * * * * * * * * * * * *$ & & & $\begin{array}{l}2 m=21 \text { veces }=42 m \\
3 m=3 \text { veces }=9 m\end{array}$ & \\
\hline & $3 m$ & $* * *$ & & & $66 \mathrm{~m}$. & \\
\hline \multirow{2}{*}{$\begin{array}{l}\text { Desplazamientos } \\
\text { hacia atrás de } \\
\text { recuperación }\end{array}$} & $1 \mathrm{~m}$ & $* * * * * * * * * * * * * * * * * *$ & $* * * *$ & \multirow{2}{*}{1 Metro } & \multirow{2}{*}{$\begin{array}{l}1 \mathrm{~m}=30 \text { veces }=30 \mathrm{~m} \\
2 \mathrm{~m}=4 \text { veces }=8 \mathrm{~m}\end{array}$} & \\
\hline & $2 m$ & $* * * *$ & & & & \\
\hline
\end{tabular}

Tabla 89. 


\begin{tabular}{|c|c|c|c|c|c|}
\hline \multicolumn{6}{|c|}{$\begin{array}{l}\text { TIPOS DE DESPLAZAMIENTOS, LOS MÁS UTILIZADOS Y SUS DISTANCIAS. PRIMER SET DEL PARTIDO DE FINAL DE GRAND SLAM WIMBLEDON } \\
\text { 2014, ENTRE NOVAK DJOKOVIC Y ROGER FEDERER. SUPERFICIE: HIERVA. }\end{array}$} \\
\hline $\begin{array}{c}\text { TIPOS DE } \\
\text { DESPLAZAMIENTOS }\end{array}$ & & TOTAL POR SET & $\begin{array}{l}\text { DISTANCIA MÁS } \\
\text { RECORRIDA }\end{array}$ & $\begin{array}{l}\text { SUMATORIA DE DISTANCIAS } \\
\text { RECORRIDAS POR SET }\end{array}$ & $\begin{array}{c}\text { TOTAL } \\
\text { RECORRIDO } \\
\end{array}$ \\
\hline \multirow{4}{*}{$\begin{array}{l}\text { Desplazamientos } \\
\quad \text { laterales }\end{array}$} & $1 \mathrm{~m}$ & $* * * * * * * * * * * * * * * * * * * * * * * * * * * * * * * * * * * * *$ & \multirow{4}{*}{1 Metro } & \multirow{3}{*}{$\begin{array}{l}1 \mathrm{~m}=37 \text { veces }=37 \mathrm{~m} \\
2 \mathrm{~m}=27 \text { veces }=54 \mathrm{~m} \\
3 \mathrm{~m}=21 \text { veces }=63 \mathrm{~m} \\
4 \mathrm{~m}=1 \text { veces }=4 \mathrm{~m}\end{array}$} & \multirow{8}{*}{$\uparrow$} \\
\hline & $2 \mathrm{~m}$ & $* * * * * * * * * * * * * * * * * * * * * * * * * * *$ & & & \\
\hline & $3 \mathrm{~m}$ & $* * * * * * * * * * * * * * * * * * * * *$ & & & \\
\hline & $4 \mathrm{~m}$ & $*$ & & $158 \mathrm{~m}$. & \\
\hline \multirow{3}{*}{$\begin{array}{l}\text { Desplazamientos } \\
\text { laterales de } \\
\text { recuperación. }\end{array}$} & $1 \mathrm{~m}$ & $\begin{array}{c}* * * * * * * * * * * * * * * * * * * * * * * * * * * * * * * * * * * * * \\
* * *\end{array}$ & \multirow{3}{*}{2 Metros } & \multirow{2}{*}{$\begin{array}{l}1 m=40 \text { veces }=40 m \\
2 m=49 \text { veces }=98 m \\
3 m=43 \text { veces }=129 m\end{array}$} & \\
\hline & $2 \mathrm{~m}$ & $\begin{array}{c}* * * * * * * * * * * * * * * * * * * * * * * * * * * * * * * * * * * * * \\
* * * * * * * * * * * *\end{array}$ & & & \\
\hline & $3 \mathrm{~m}$ & $\begin{array}{c}* * * * * * * * * * * * * * * * * * * * * * * * * * * * * * * * * * * * \\
* * * * * *\end{array}$ & & $267 m$. & \\
\hline $\begin{array}{l}\text { Desplazamientos } \\
\text { hacia arriba o saltos }\end{array}$ & \multicolumn{2}{|c|}{$\begin{array}{c}* * * * * * * * * * * * * * * * * * * * * * * * * * * * * * * * * * * * * * * * * * * \\
* * * * * * * * * * * * * * * * * * * * * * * * * * * * * * * * * * * * * * * * * * \\
* * * * * * * * * * * * * * *\end{array}$} & \multicolumn{2}{|c|}{ Saltos en servicio $=109$ veces } & \\
\hline
\end{tabular}

Tabla 90.

\begin{tabular}{|c|c|c|c|c|c|c|}
\hline \multicolumn{7}{|c|}{$\begin{array}{l}\text { PORCENTAJES DE USO DE LOS DIFERENTES TIPOS DE DESPLAZAMIENTOS EN EL PRIMER SET DEL PARTIDO DE FINAL DE GRAND SLAM } \\
\text { WIMBLEDON 2014, ENTRE NOVAK DJOKOVIC Y ROGER FEDERER. SUPERFICIE: HIERVA. }\end{array}$} \\
\hline & $\begin{array}{l}\text { D HACIA } \\
\text { ADELANTE }\end{array}$ & $\begin{array}{l}\text { D HACIA ATRÁS } \\
\text { DE EJECUCIÓN }\end{array}$ & $\begin{array}{l}\text { D HACIA ATRÁS DE } \\
\text { RECUPERACIÓN }\end{array}$ & D LATERALES & $\begin{array}{l}\text { D LATERALES DE } \\
\text { RECUPERACIÓN }\end{array}$ & $\begin{array}{l}\text { D HACIA } \\
\text { ARRIBA }\end{array}$ \\
\hline NÚMERO DE VECES & $40,38 \%$ & $5,81 \%$ & $5,06 \%$ & $12,81 \%$ & $19,67 \%$ & $16,24 \%$ \\
\hline DISTANCIA & $61,99 \%$ & $4,74 \%$ & $2,72 \%$ & $11,35 \%$ & $19,18 \%$ & \\
\hline
\end{tabular}

Tabla 91. 


\begin{tabular}{|c|c|c|c|c|}
\hline \multicolumn{5}{|c|}{$\begin{array}{l}\text { TIEMPOS GLOBALES DE LOS MOMENTOS DE TRABAJO Y PAUSA DEL PRIMER SET DEL PARTIDO } \\
\text { ENTRE NOVAK DJOKOVIC Y ROGER FEDERER EN LA FINAL DE WIMBLEDON } 2014\end{array}$} \\
\hline TIEMPOS & $\begin{array}{l}\text { SUMATO } \\
\text { TIEMPOS PC }\end{array}$ & $\begin{array}{l}\text { IA DE } \\
\text { R GAME }\end{array}$ & $\begin{array}{l}\text { SUMATORIA DE TIEMPOS } \\
\text { POR SET }\end{array}$ & $\begin{array}{l}\text { RELACIÓN TIEMPO } \\
\text { DE TRABAJO-PAUSA }\end{array}$ \\
\hline \multirow{13}{*}{ TIEMPO DE TRABAJO } & $1^{\mathrm{er}}$ Game & $33,65^{\prime \prime}$ & \multirow{13}{*}{$\begin{array}{l}485,98 " \text { tiempo real de } \\
\text { trabajo }\end{array}$} & \multirow{26}{*}{$\begin{array}{l}1 " \text { de trabajo } \\
X \\
5,07 " \text { de pausa }\end{array}$} \\
\hline & $2^{\text {do }}$ Game & $37.37^{\prime \prime}$ & & \\
\hline & $3^{\text {er }}$ Game & $16,37^{\prime \prime}$ & & \\
\hline & $4^{\text {to }}$ Game & $26,98^{\prime \prime}$ & & \\
\hline & $5^{\text {to }}$ Game & $14,61^{\prime \prime}$ & & \\
\hline & $6^{\text {to }}$ Game & $12,26^{\prime \prime}$ & & \\
\hline & $7^{\text {to }}$ Game & $19,23^{\prime \prime}$ & & \\
\hline & $8^{\text {vo }}$ Game & $37,59 "$ & & \\
\hline & $9^{\text {no }}$ Game & $44,76^{\prime \prime}$ & & \\
\hline & $10^{\mathrm{mo}}$ Game & $79,54 "$ & & \\
\hline & $11^{\text {vo }}$ Game & $48,41^{\prime \prime}$ & & \\
\hline & $12^{\text {vo }}$ Game & $24,69 "$ & & \\
\hline & Tie Break & $115,27^{\prime \prime}$ & & \\
\hline \multirow{13}{*}{ TIEMPO DE DESCANSO } & $1^{\mathrm{er}}$ Game & $114,56^{\prime \prime}$ & \multirow{13}{*}{$\begin{array}{l}2.468,34 " \text { tiempo real de } \\
\text { Descanso }\end{array}$} & \\
\hline & $2^{\text {do }}$ Game & $152,34^{\prime \prime}$ & & \\
\hline & $3^{\text {er }}$ Game & $143,89^{\prime \prime}$ & & \\
\hline & $4^{\text {to }}$ Game & $110,81^{\prime \prime}$ & & \\
\hline & $5^{\text {to }}$ Game & $143,75^{\prime \prime}$ & & \\
\hline & $6^{\text {to }}$ Game & $103,63^{\prime \prime}$ & & \\
\hline & $7^{\text {to }}$ Game & $161,68^{\prime \prime}$ & & \\
\hline & $8^{\text {vo }}$ Game & $157,88^{\prime \prime}$ & & \\
\hline & $9^{\text {no }}$ Game & $204.5^{\prime \prime}$ & & \\
\hline & $10^{\text {mo }}$ Game & $188,96^{\prime \prime}$ & & \\
\hline & $11^{\text {vo }}$ Game & $248,2^{\prime \prime}$ & & \\
\hline & $12^{\text {vo }}$ Game & $111,87^{\prime \prime}$ & & \\
\hline & Tie Break & $618,76^{\prime \prime}$ & & \\
\hline
\end{tabular}

Tabla 92. 


\begin{tabular}{|c|c|c|c|}
\hline \multicolumn{4}{|c|}{$\begin{array}{l}\text { PROMEDIO DE LOS MOMENTOS ESPECÍFICOS DE TRABAJO Y PAUSA DEL PRIMER SET DEL PARTIDO } \\
\text { ENTRE NOVAK DJOKOVIC Y ROGER FEDERER EN LA FINAL DE WIMBLEDON } 2014\end{array}$} \\
\hline \multicolumn{2}{|c|}{ PROMEDIOS DE TRABAJO } & \multicolumn{2}{|c|}{ PROMEDIOS DE PAUSA } \\
\hline $1^{\text {er }}$ Game & $6,73^{\prime \prime}$ & $1^{\text {er }}$ Game & $18,28^{\prime \prime}$ \\
\hline $2^{\text {do }}$ Game & $4,67^{\prime \prime}$ & $2^{\text {do }}$ Game & $17,67^{\prime \prime}$ \\
\hline $3^{\text {er }}$ Game & $4,09^{\prime \prime}$ & $3^{\text {er }}$ Game & $17,96^{\prime \prime}$ \\
\hline $4^{\text {to }}$ Game & $5,39^{\prime \prime}$ & $4^{\text {to }}$ Game & $27,43^{\prime \prime}$ \\
\hline $5^{\text {to }}$ Game & $3,65^{\prime \prime}$ & $5^{\text {to }}$ Game & $16,91^{\prime \prime}$ \\
\hline $6^{\text {to }}$ Game & $2,04^{\prime \prime}$ & $6^{\text {to }}$ Game & $14,69^{\prime \prime}$ \\
\hline $7^{\mathrm{mo}}$ Game & $3,84^{\prime \prime}$ & $7^{\mathrm{mo}}$ Game & $16,92^{\prime \prime}$ \\
\hline $8^{\text {vo }}$ Game & 4,69" & $8^{\text {vo }}$ Game & $17,34^{\prime \prime}$ \\
\hline $9^{\text {no }}$ Game & $7,46^{\prime \prime}$ & $9^{\text {no }}$ Game & $22,1^{\prime \prime}$ \\
\hline $10^{\text {mo }}$ Game & 9,94" & $10^{\text {mo }}$ Game & $22 "$ \\
\hline $11^{\text {vo }}$ Game & $6,05^{\prime \prime}$ & $11^{\text {vo }}$ Game & $22,17^{\prime \prime}$ \\
\hline $12^{\mathrm{vo}}$ Game & $4,11^{\prime \prime}$ & $12^{\mathrm{vo}}$ Game & $16,65^{\prime \prime}$ \\
\hline Tie Break & $7,20^{\prime \prime}$ & Tie Break & $29,51^{\prime \prime}$ \\
\hline \multicolumn{2}{|c|}{ Promedio total } & \multicolumn{2}{|c|}{ Promedio total } \\
\hline \multicolumn{2}{|c|}{$5,37^{\prime \prime}$} & \multicolumn{2}{|c|}{$19,97^{\prime \prime}$} \\
\hline \multicolumn{4}{|c|}{ 1" de trabajo por $3,71 "$ de descanso } \\
\hline
\end{tabular}

Tabla 93.

6. Análisis punto a punto del primer set del partido entre Eugénie Bouchard (Fra) y Petra Kvitova (RCh) en la final del Grand Slam Wimbledom 2014. Y en la que $P$ Kvitova le gana a E Bouchard, 6-3, 6-0. Tipo de pista o Cancha (hierba). clasificación ITF categoría 5 (velocidad alta). Ver tablas 95 a108.

- Análisis de los tiempos y tipos de golpes ejecutados dentro de la cancha antes de comenzar el partido (peloteo previo al partido).

Las jugadoras después de entrar a la cancha y realizar los actos protocolarios de fotografías y sorteo para la elección de servicio y cancha, comienzan a pelotear detrás de la línea media de la línea de fondo, a una intensidad media sin realizarse grandes desplazamientos, a lo sumo 1 o 2 metros. Al minuto 1,25' Petra Kvitova se sitúa cerca a la red para volear hasta el minuto 2' y luego hacer Smash sobre la "T" hasta el minuto 2,12', mientras Eugene Bouchard sigue peloteando dese el fondo de la cancha. Al minuto 2,15' se cambian las posiciones y E Bouchard sube a volear hasta el minuto 2,50', y luego Smash 
hasta el minuto 3', en el que las dos empiezan hacer servicios desde el fondo de la cancha hasta el minuto 5,20' y luego se sientan a descansar 42" para luego levantarse y comenzar el partido. Ver tabla 94.

\begin{tabular}{|c|c|c|c|c|}
\hline \multicolumn{5}{|c|}{$\begin{array}{c}\text { ANÁLISIS DEL USO DE LOS TIPOS DE GOLPES EN MOMENTO PREVIO AL COMIENZO } \\
\text { DEL PARTIDO(PELOTEO PREVIO AL PARTIDO) }\end{array}$} \\
\hline Tipo de golpe & E Bouchard & P Kvitova & Tiempo promedio & $\%$ \\
\hline $\begin{array}{l}\text { (derecha y revés) Peloteo } \\
\text { de fondo de cancha }\end{array}$ & $135 "$ & $\begin{array}{c}85 "+35 "+ \\
10 " \\
=130 "\end{array}$ & $132,5^{\prime \prime}$ & $41,60 \%$ \\
\hline Volea cerca a la red & $35 "$ & $35 "$ & $35 "$ & $10,98 \%$ \\
\hline Smash sobre la "T" & $10 "$ & $12 "$ & $11 "$ & $3,45 \%$ \\
\hline Servicio o saque & $140 "$ & $140 "$ & $140 "$ & $43,95 \%$ \\
\hline
\end{tabular}

Tabla 94.

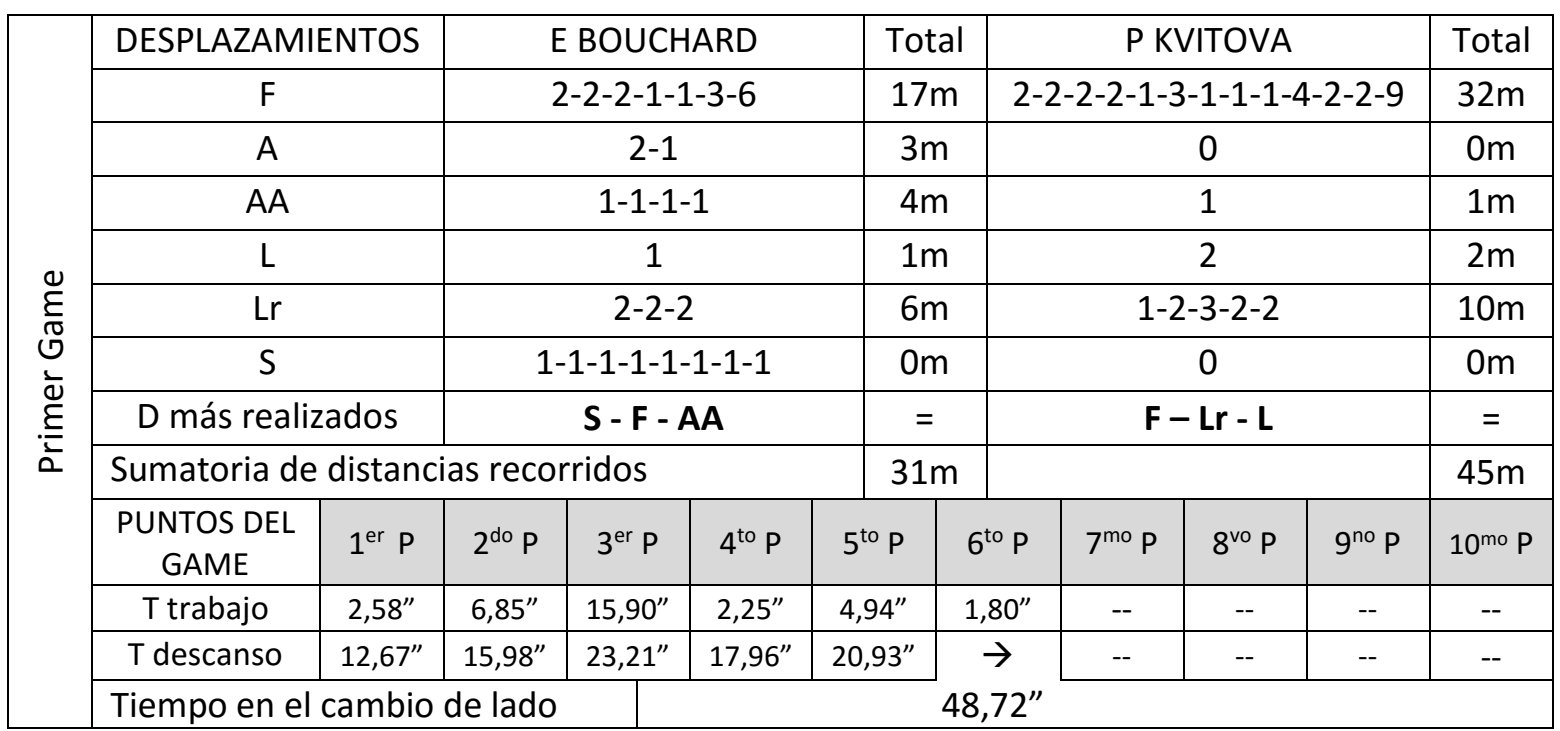

Tabla 95. 


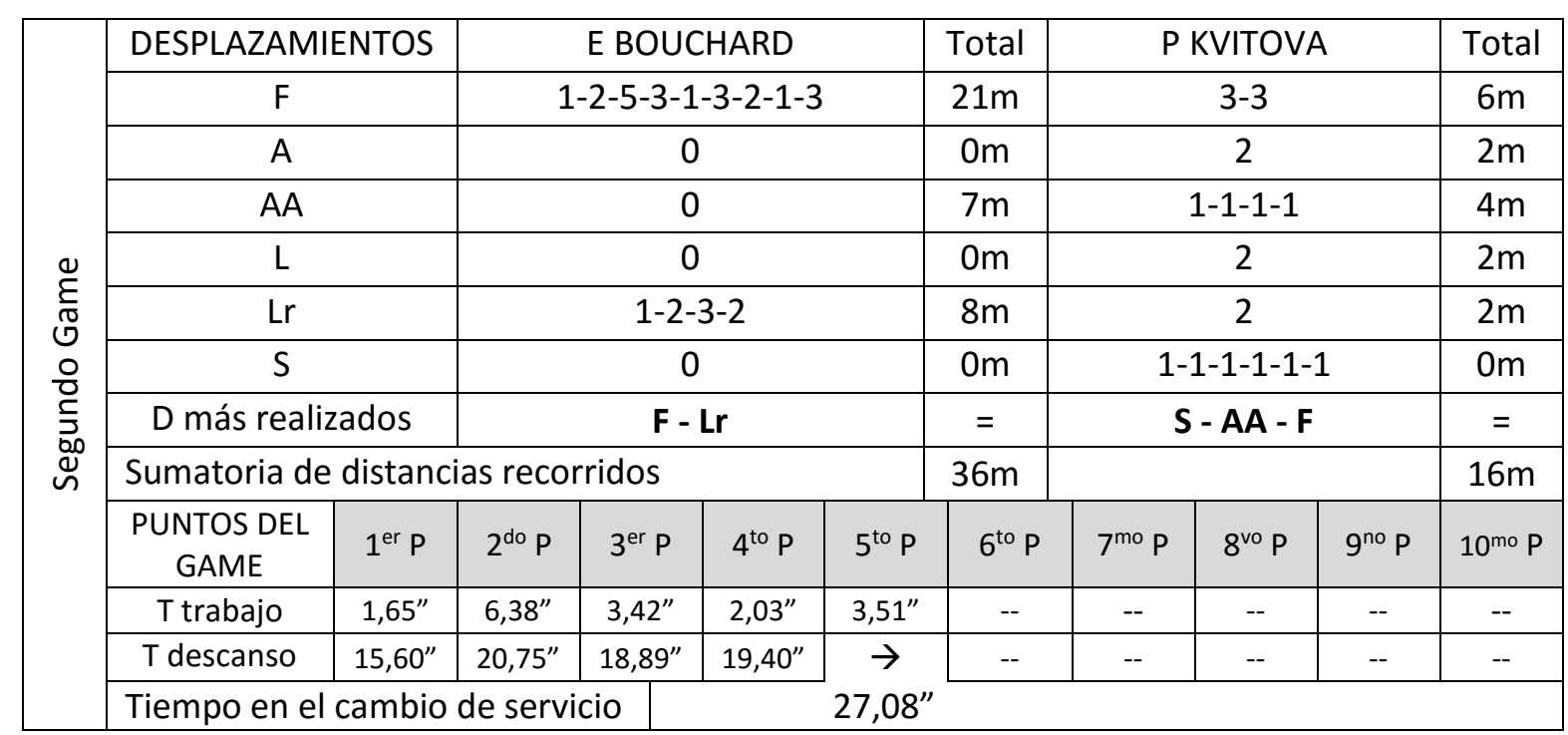

\section{Tabla 96.}

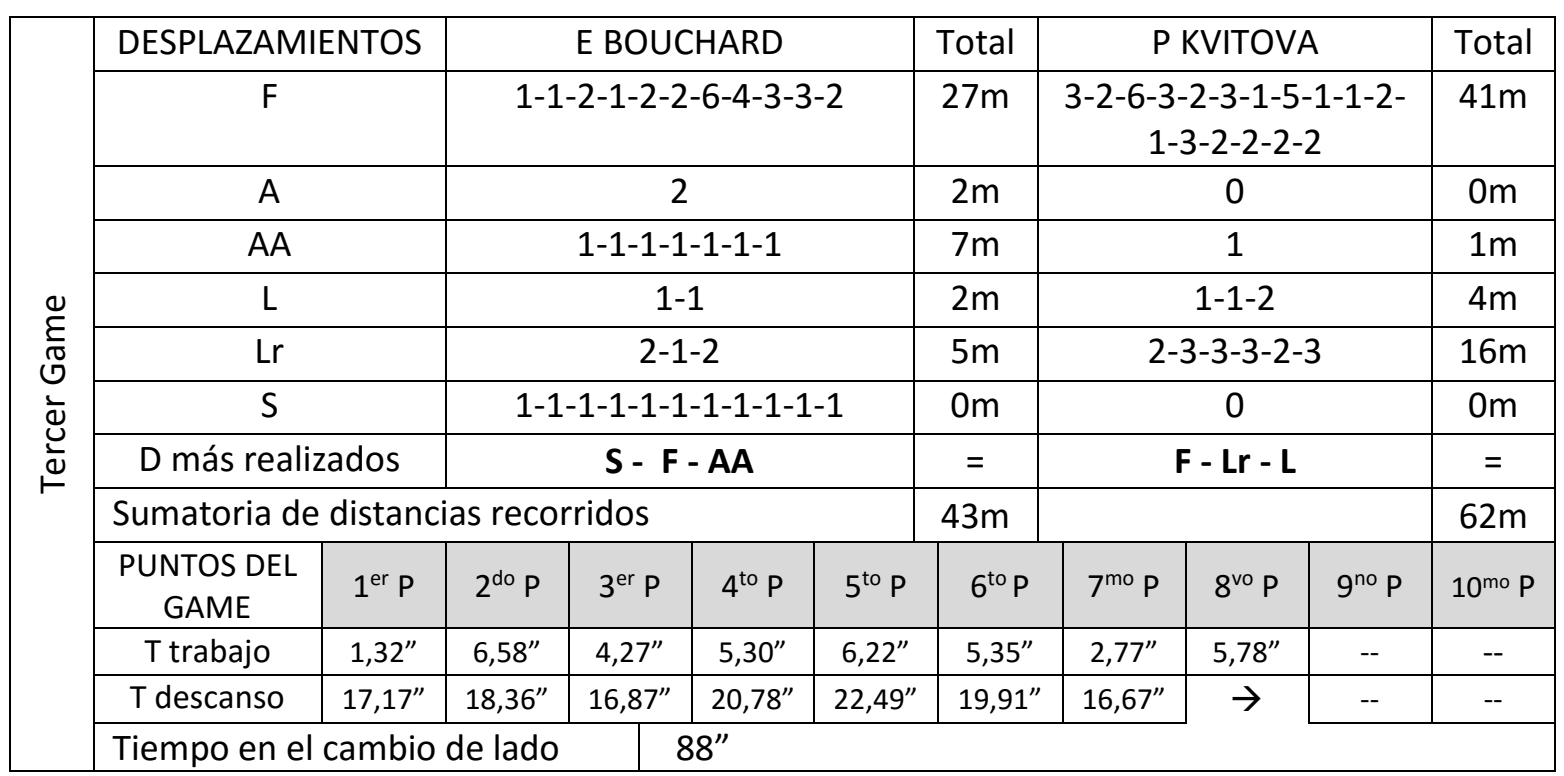

\section{Tabla 97.}




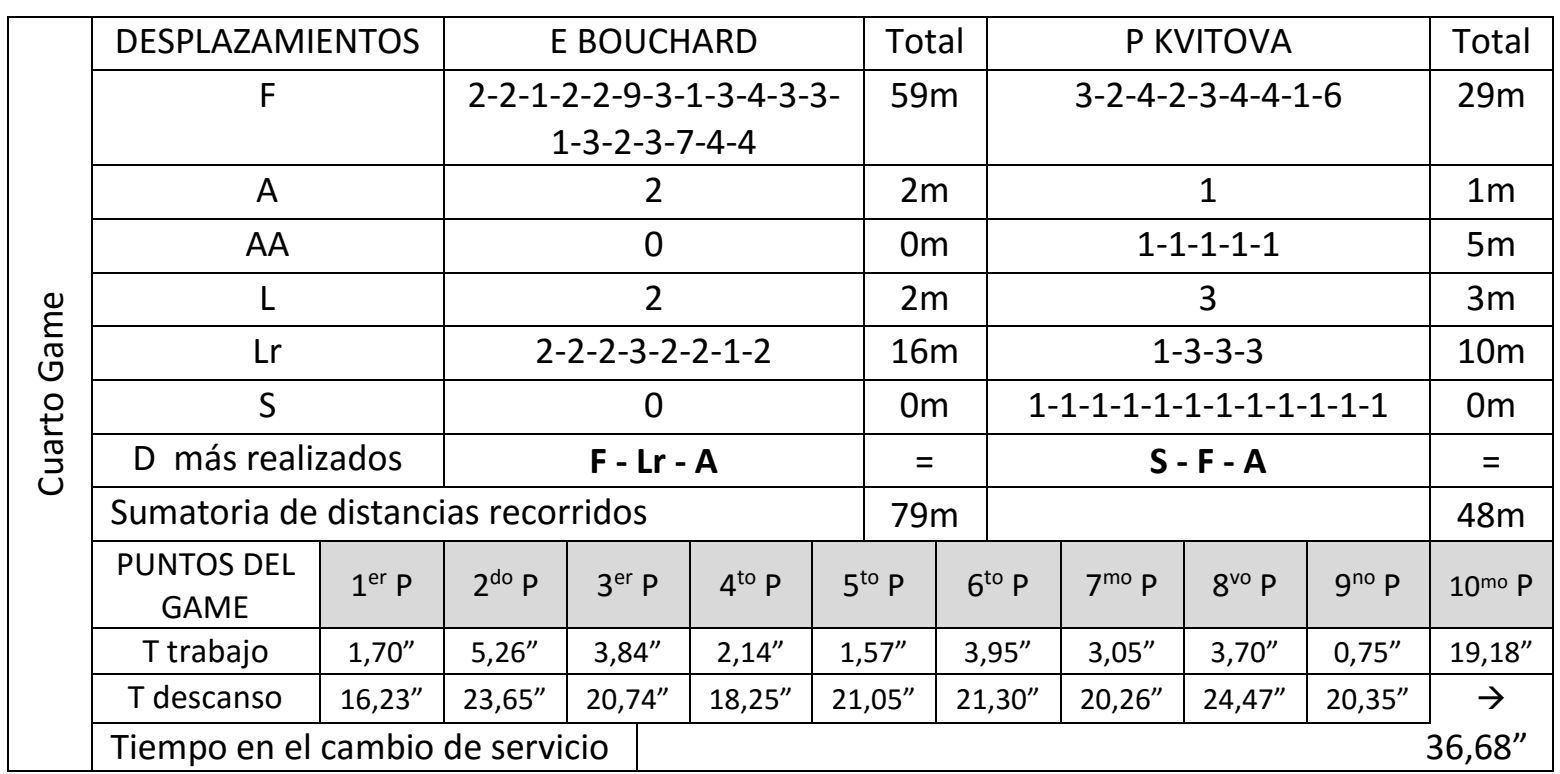

\section{Tabla 98.}

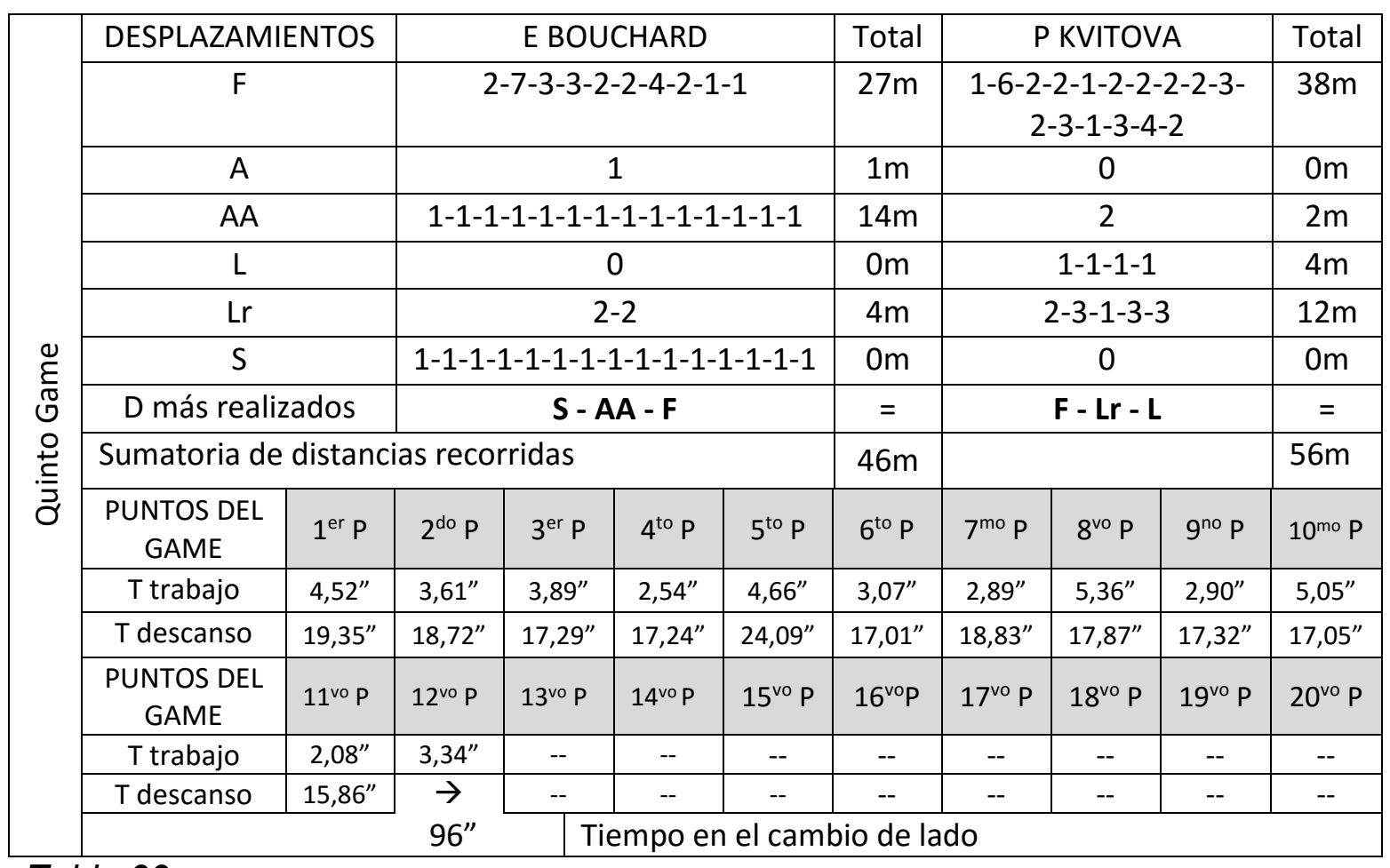

Tabla 99. 


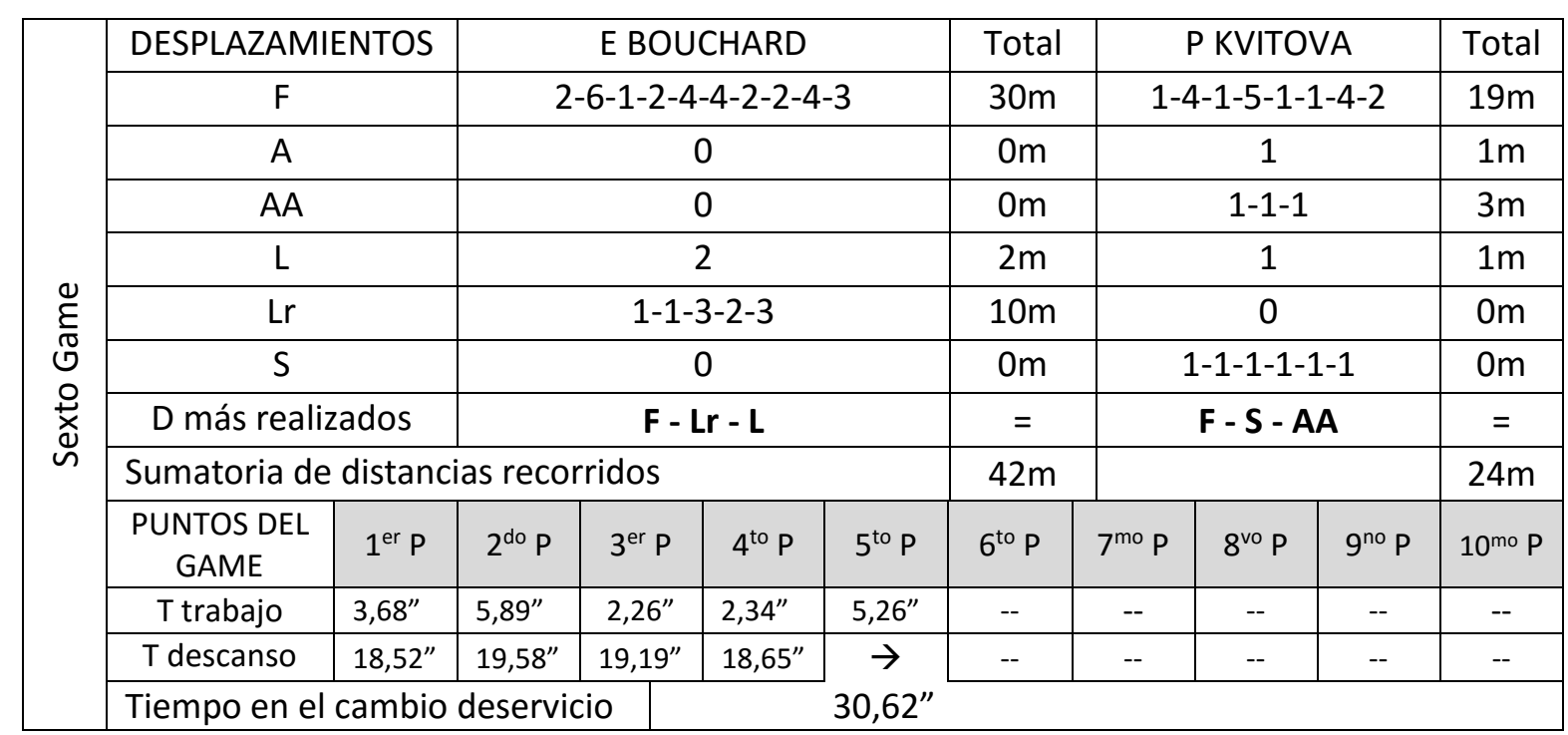

Tabla 100.

\begin{tabular}{|c|c|c|c|c|c|c|c|c|c|c|c|}
\hline \multirow{13}{*}{ 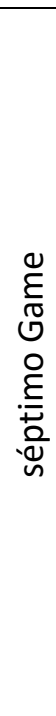 } & \multicolumn{2}{|c|}{ DESPLAZAMIENTOS } & \multicolumn{4}{|c|}{ E BOUCHARD } & Total & \multicolumn{3}{|c|}{ P KVITOVA } & Total \\
\hline & \multicolumn{2}{|l|}{$\mathrm{F}$} & \multicolumn{4}{|c|}{$2-5-3-3-2$} & $15 \mathrm{~m}$ & \multicolumn{3}{|c|}{$2-4-4-2$} & $12 m$ \\
\hline & \multicolumn{2}{|l|}{ A } & \multicolumn{4}{|c|}{$1-2$} & $3 m$ & \multicolumn{3}{|c|}{0} & $0 m$ \\
\hline & \multicolumn{2}{|l|}{ AA } & \multicolumn{4}{|c|}{ 1-1-1-1 } & $4 m$ & \multicolumn{3}{|c|}{1} & $1 \mathrm{~m}$ \\
\hline & \multicolumn{2}{|l|}{$\mathrm{L}$} & \multicolumn{4}{|c|}{0} & $0 m$ & \multicolumn{3}{|c|}{1} & $1 \mathrm{~m}$ \\
\hline & \multicolumn{2}{|l|}{ Lr } & \multicolumn{4}{|c|}{$2-2-1$} & $5 m$ & \multicolumn{3}{|c|}{$1-2-1-1$} & $5 m$ \\
\hline & \multicolumn{2}{|l|}{$S$} & \multicolumn{4}{|c|}{ 1-1-1-1-1-1 } & $0 \mathrm{~m}$ & \multicolumn{3}{|c|}{0} & $0 m$ \\
\hline & \multicolumn{2}{|c|}{ D más realizados } & \multicolumn{4}{|c|}{$S-A A-L r$} & $=$ & \multicolumn{3}{|c|}{$L r-F-L$} & $=$ \\
\hline & \multicolumn{6}{|c|}{ Sumatoria de distancias recorridos } & $27 \mathrm{~m}$ & & & & $19 m$ \\
\hline & $\begin{array}{l}\text { PUNTOS DEL } \\
\text { GAME }\end{array}$ & $1^{\text {er }} \mathrm{P}$ & $2^{\text {do }} \mathrm{P}$ & $3^{\text {er } P}$ & $4^{\text {to }} \mathrm{P}$ & $5^{\text {to }} P$ & $6^{\text {to }} \mathrm{p}$ & $7^{\text {mo }} \mathrm{p}$ & $8^{\text {vo }} \mathrm{P}$ & $9^{\text {no }} P$ & $10^{\text {mo }} \mathrm{P}$ \\
\hline & $\mathrm{T}$ trabajo & $4,16^{\prime \prime}$ & $4,13^{\prime \prime}$ & $2,90^{\prime \prime}$ & $1,97^{\prime \prime}$ & $5,22^{\prime \prime}$ & -- & -- & -- & -- & -- \\
\hline & T descanso & $18,06^{\prime \prime}$ & $18,39^{\prime \prime}$ & $17,08^{\prime \prime}$ & $21,97 "$ & $\rightarrow$ & -- & - & -- & - & -- \\
\hline & \multicolumn{5}{|c|}{ Tiempo en el cambio de lado } & $90 "$ & & & & & \\
\hline
\end{tabular}

Tabla 101. 


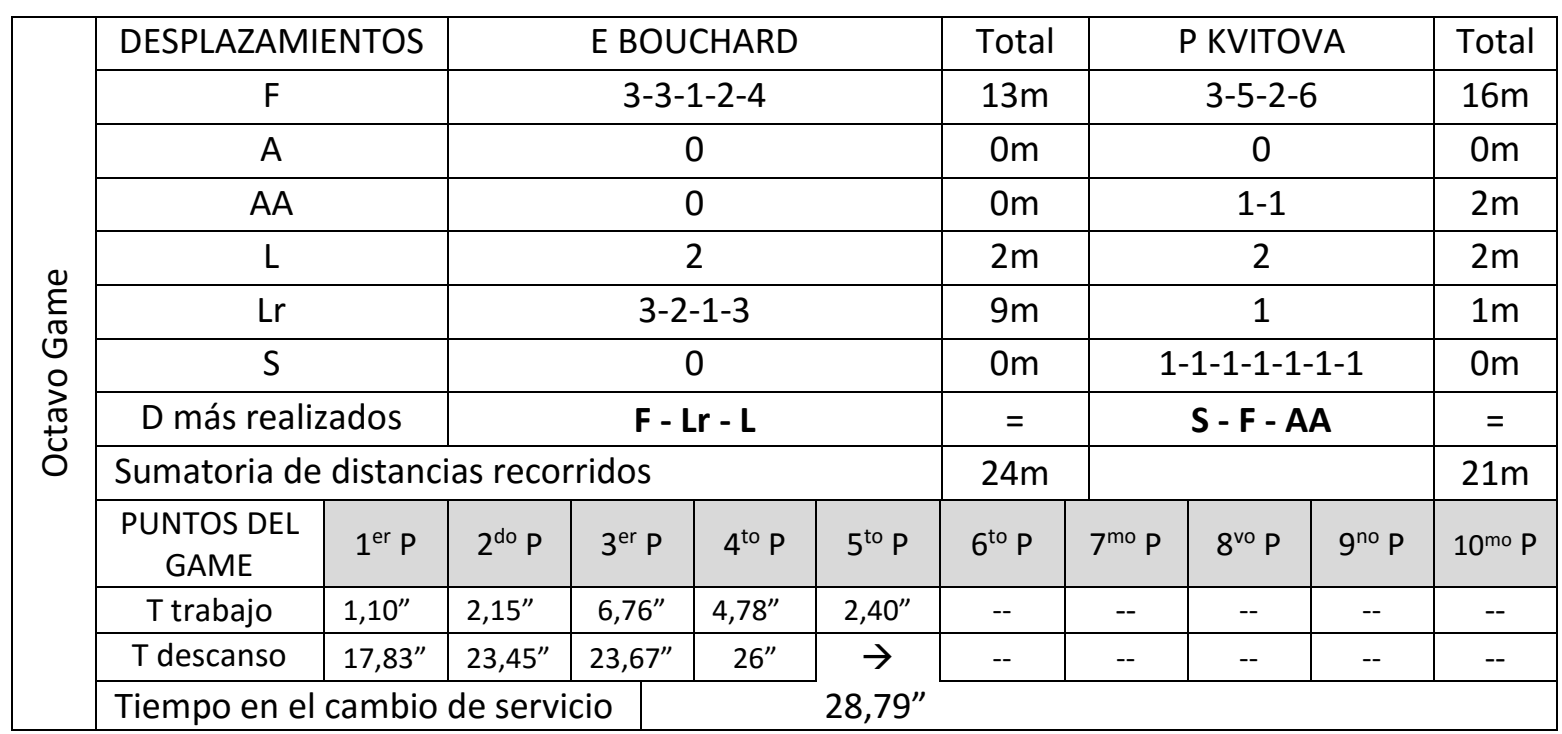

Tabla 102.

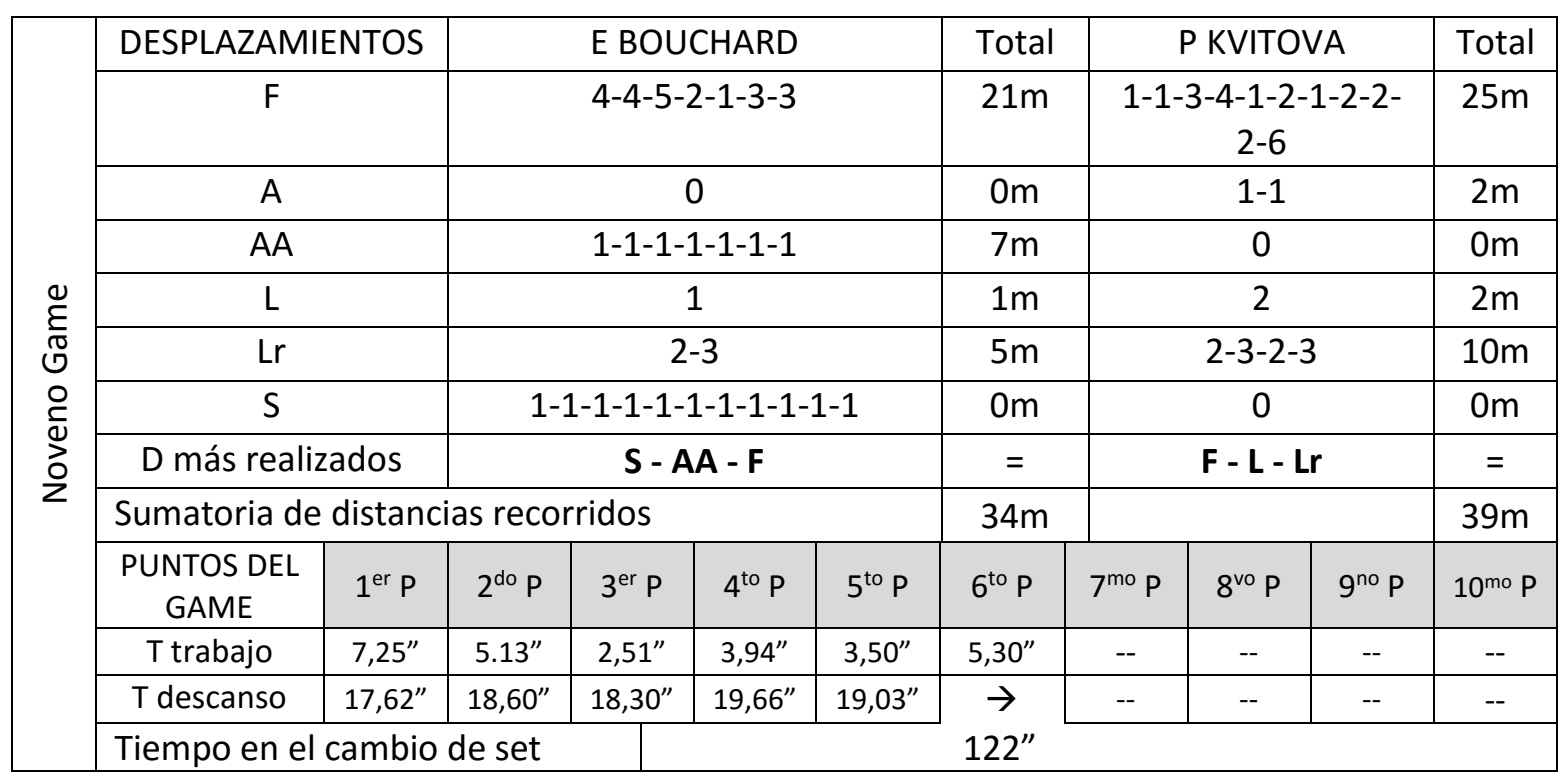

Tabla 103. 


\begin{tabular}{|c|c|c|c|c|c|}
\hline \multicolumn{6}{|c|}{$\begin{array}{l}\text { TIPOS DE DESPLAZAMIENTOS, LOS MÁS UTILIZADOS Y SUS DISTANCIAS. PRIMER SET DEL PARTIDO DE FINAL DE GRAND SLAM WIMBLEDON } \\
\text { 2014, ENTRE EUGÉNIE BOUCHARD Y PETRA KVITOVA. SUPERFICIE: HIERVA. }\end{array}$} \\
\hline $\begin{array}{l}\text { TIPOS DE } \\
\text { DESPLAZAMIENTOS }\end{array}$ & & TOTAL POR SET & $\begin{array}{l}\text { DISTANCIA MÁS } \\
\text { RECORRIDA }\end{array}$ & $\begin{array}{l}\text { SUMATORIA DE DISTANCIAS } \\
\text { RECORRIDAS POR SET }\end{array}$ & $\begin{array}{l}\text { TOTAL } \\
\text { RECORRIDO }\end{array}$ \\
\hline \multirow{7}{*}{$\begin{array}{l}\text { Desplazamientos de } \\
\quad \text { frente }\end{array}$} & $1 \mathrm{~m}$ & $* * * * * * * * * * * * * * * * * * * * * * * * * * * * * * * * * * * * * * *$ & \multirow{7}{*}{2 Metros } & \multirow{7}{*}{$\begin{array}{l}1 \mathrm{~m}=36=36 \mathrm{~m} . \\
2 \mathrm{~m}=57 \text { veces }=114 \mathrm{~m} . \\
3 \mathrm{~m}=32 \text { veces }=96 \mathrm{~m} \\
4 \mathrm{~m}=18 \text { veces }=72 \mathrm{~m} \\
5 \mathrm{~m}=6 \text { veces }=30 \mathrm{~m} \\
6 \mathrm{~m}=7 \text { veces }=42 \mathrm{~m} \\
9 \mathrm{~m}=2 \text { veces }=18 \mathrm{~m} \\
408 \mathrm{~m} .\end{array}$} & \multirow{10}{*}{$601 \mathrm{~m}}$. \\
\hline & $2 \mathrm{~m}$ & $\begin{array}{c}* * * * * * * * * * * * * * * * * * * * * * * * * * * * * * * * * * * * *) \\
* * * * * * * * * * * * * * * * * * * * *\end{array}$ & & & \\
\hline & $3 m$ & $* * * * * * * * * * * * * * * * * * * * * * * * * * * * * * * * * *$ & & & \\
\hline & $4 \mathrm{~m}$ & $* * * * * * * * * * * * * * * * * *$ & & & \\
\hline & $5 \mathrm{~m}$ & $* * * * * *$ & & & \\
\hline & $6 \mathrm{~m}$ & $* * * * * * *$ & & & \\
\hline & $9 m$ & $* *$ & & & \\
\hline \multirow{2}{*}{$\begin{array}{l}\text { Desplazamientos } \\
\text { hacia atrás de } \\
\text { recuperación }\end{array}$} & $1 \mathrm{~m}$ & $* * * * * * * *$ & \multirow{2}{*}{1 Metro } & $\begin{array}{l}1 \mathrm{~m}=7 \text { veces }=7 \mathrm{~m} \\
2 \mathrm{~m}=5 \text { veces }=10 \mathrm{~m}\end{array}$ & \\
\hline & $2 m$ & $* * * * *$ & & $17 \mathrm{~m}$. & \\
\hline $\begin{array}{l}\text { Desplazamientos } \\
\text { hacia atrás de } \\
\text { recuperación }\end{array}$ & $1 \mathrm{~m}$ & $\begin{array}{c}* * * * * * * * * * * * * * * * * * * * * * * * * * * * * * * * * * * * * * \\
* * * * * * * * * * * * * * * * *\end{array}$ & 1 Metro & $\begin{array}{c}1 \mathrm{~m}=53 \text { veces }=53 \mathrm{~m} . \\
\mathbf{5 3 m} .\end{array}$ & \\
\hline
\end{tabular}

Tabla 104. 


\begin{tabular}{|c|c|r|r|c|}
\hline \multicolumn{3}{|c|}{ TIPOS DE DESPLAZAMIENTOS, LOS MÁS UTILIZADOS Y SUS DISTANCIAS. PRIMER SET DEL PARTIDO DE FINAL DE GRAND SLAM WIMBLEDON } \\
2014, ENTRE EUGÉNIE BOUCHARD Y PETRA KVITOVA. SUPERICIE: HIERVA.
\end{tabular}

Tabla 105.

\begin{tabular}{|c|c|c|c|c|c|c|}
\hline \multicolumn{7}{|c|}{ PORCENTAJES DE USO DE LOS DIFERENTES TIPOS DE DESPLAZAMIENTOS EN EL PRIMER SET DEL PARTIDO DE FINAL DE GRAND SLAM } \\
WIMBLEDON 2014, ENTRE EUGÉNIE BOUCHARD Y PETRA KVITOVA. SUPERFICIE: HIERVA.
\end{tabular}

Tabla 106. IMBLEDON 2014, ENTRE EUGÉNIE BOUCHARD Y PETRA KVITOVA. SUPERFICIE: HIERVA. 


\begin{tabular}{|c|c|c|c|c|}
\hline \multicolumn{5}{|c|}{$\begin{array}{l}\text { TIEMPOS GLOBALES DE LOS MOMENTOS DE TRABAJO Y PAUSA DEL PRIMER SET DEL PARTIDO } \\
\text { ENTRE EUGÉNIE BOUCHARD Y PETRA KVITOVA EN LA FINAL DE WIMBLEDON } 2014\end{array}$} \\
\hline TIEMPOS & \multicolumn{2}{|c|}{$\begin{array}{l}\text { SUMATORIA DE } \\
\text { TIEMPOS POR GAME }\end{array}$} & $\begin{array}{l}\text { SUMATORIA DE TIEMPOS } \\
\text { POR SET }\end{array}$ & $\begin{array}{l}\text { RELACIÓN TIEMPO } \\
\text { DE TRABAJO-PAUSA }\end{array}$ \\
\hline \multirow{9}{*}{ TIEMPO DE TRABAJO } & $1^{\text {er }}$ Game & $34,32^{\prime \prime}$ & \multirow{9}{*}{$\begin{array}{l}260,3^{\prime \prime} \text { tiempo real de } \\
\text { trabajo }\end{array}$} & \multirow{18}{*}{$\begin{array}{l}1 " \text { de trabajo } \\
\quad \times \\
6,1^{\prime \prime} \text { de pausa }\end{array}$} \\
\hline & $2^{\text {do }}$ Game & $16,99^{\prime \prime}$ & & \\
\hline & $3^{\text {er }}$ Game & $37,59^{\prime \prime}$ & & \\
\hline & $4^{\text {to }}$ Game & $45,14^{\prime \prime}$ & & \\
\hline & $5^{\text {to }}$ Game & $43,96 "$ & & \\
\hline & $6^{\text {to }}$ Game & $19,43^{\prime \prime}$ & & \\
\hline & $7^{\text {to }}$ Game & $18,38^{\prime \prime}$ & & \\
\hline & $8^{\text {vo }}$ Game & $17,19^{\prime \prime}$ & & \\
\hline & $9^{\text {no }}$ Game & $27,63^{\prime \prime}$ & & \\
\hline \multirow{9}{*}{ TIEMPO DE DESCANSO } & $1^{\mathrm{er}}$ Game & $139,48^{\prime \prime}$ & \multirow{9}{*}{$\begin{array}{l}1588,07 " \text { tiempo real de } \\
\text { Descanso }\end{array}$} & \\
\hline & $2^{\text {do }}$ Game & $101,72^{\prime \prime}$ & & \\
\hline & $3^{\text {er }}$ Game & $220,25^{\prime \prime}$ & & \\
\hline & $4^{\text {to }}$ Game & $222,98^{\prime \prime}$ & & \\
\hline & $5^{\text {to }}$ Game & 296,63" & & \\
\hline & $6^{\text {to }}$ Game & $106,56^{\prime \prime}$ & & \\
\hline & $7^{\text {to }}$ Game & $165,5^{\prime \prime}$ & & \\
\hline & $8^{\text {vo }}$ Game & $119,74^{\prime \prime}$ & & \\
\hline & $9^{\text {no }}$ Game & $215,21^{\prime \prime}$ & & \\
\hline
\end{tabular}

Tabla 107.

PROMEDIO DE LOS MOMENTOS ESPECÍFICOS DE TRABAJO Y PAUSA DEL PRIMER SET DEL PARTIDO ENTRE EUGÉNIE BOUCHARD Y PETRA KVITOVA EN LA FINAL DE WIMBLEDON 2014

\begin{tabular}{|c|c|c|c|}
\hline \multicolumn{2}{|c|}{ PROMEDIOS DE TRABAJO } & \multicolumn{2}{|c|}{ PROMEDIOS DE PAUSA } \\
\hline $1^{\text {er }}$ Game & $5,72^{\prime \prime}$ & $1^{\text {er }}$ Game & $18,15^{\prime \prime}$ \\
\hline $2^{\text {do }}$ Game & $3,39^{\prime \prime}$ & $2^{\text {do }}$ Game & $18,66^{\prime \prime}$ \\
\hline $3^{\text {er }}$ Game & $4,69 "$ & $3^{\text {er }}$ Game & $18,89^{\prime \prime}$ \\
\hline $4^{\text {to }}$ Game & $4,51^{\prime \prime}$ & $4^{\text {to }}$ Game & $20,7^{\prime \prime}$ \\
\hline $5^{\text {to }}$ Game & $3,66^{\prime \prime}$ & $5^{\text {to }}$ Game & $18,23^{\prime \prime}$ \\
\hline $6^{\text {to }}$ Game & $3,88^{\prime \prime}$ & $6^{\text {to }}$ Game & $18,98^{\prime \prime}$ \\
\hline $7^{\mathrm{mo}}$ Game & $3,67^{\prime \prime}$ & $7^{\mathrm{mo}} \mathrm{Game}$ & $18,87^{\prime \prime}$ \\
\hline $8^{\text {vo }}$ Game & $3,43^{\prime \prime}$ & $8^{\text {vo }}$ Game & $22,74^{\prime \prime}$ \\
\hline $9^{\text {no }}$ Game & $4,60^{\prime \prime}$ & $9^{\text {no }}$ Game & $18,84^{\prime \prime}$ \\
\hline \multicolumn{2}{|c|}{ Promedio total } & \multicolumn{2}{|c|}{ Promedio total } \\
\hline \multicolumn{2}{|c|}{$4,17^{\prime \prime}$} & \multicolumn{2}{|c|}{$19,34^{\prime \prime}$} \\
\hline & det & descans & \\
\hline
\end{tabular}

Tabla 108. 


\section{RESULTADOS DE LOS ANÁLISIS DE LAS COMPETICIONES.}

Desde estos análisis de las observaciones que se realizaron a las competencias de más alto nivel mundial, pretendemos mostrar que de acuerdo a los diferentes tipos de superficie donde se juega al tenis, hay diferentes tipos de exigencias físicas que los deportistas deben afrontar,

\subsection{Tipos de desplazamientos más utilizados en la competición.}

Para comenzar a describir cuales son los tipos de desplazamiento más utilizados en una competencia tenística, tenemos que describir primero a qué tipos de desplazamientos nos estamos refiriendo. Para ello, en esta tesis, hemos tomado como tipos de desplazamiento, cuatro tipos que son: $F$ (de frente o hacia delante), A (hacia atrás), AA (hacia atrás de acomodación), L (lateral), Lr (lateral de recuperación) y S (servicio o smash). Podemos ver que hay dos tipos de desplazamientos "laterales" y dos tipos de desplazamientos "hacia atrás", esto es porque en el tenis, los desplazamientos no solamente se hacen para ir a impactar la pelota, sino que se utilizan también para acomodarse en un mejor lugar, según como venga el juego o peloteo; también se utiliza para recuperar la posición de la que tuvo que salir para ir a golpear una pelota y así no dejar gran parte de la cancha descubierta.

Es así, que tomamos estas dos variantes de desplazamientos, si pudiéramos llamarla así, para designar el mismo tipo de desplazamiento, pero más específico para el estudio de acuerdo a su utilización. Esta utilización de cada tipo de desplazamiento, es una buena pauta para poder sacar conclusiones de cuál es el porcentaje en que se utiliza cada tipo de desplazamiento en un partido, qué tipo de desplazamiento utiliza con frecuencia un jugador, cuáles son los desplazamientos más rápidos, cuáles son los desplazamientos más lentos y cuáles son los desplazamientos más utilizados para ir a impactar una pelota. Todo esto entonces haría que una preparación para el deportista, sea 
más específica y se prepare para lo que, en probabilidad, se hace en un partido.

Esta tesis ha tomado como referente para su estudio, 6 partidos diferentes, todos de alto nivel (4 de Grand Slam, un Master mil y un ATP 250), en tres diferentes tipos de superficie (polvo de ladrillo, hierba y cemento); damas, uno en cada superficie y, caballeros, uno en cada superficie. Teniendo en cuenta que cada superficie aporta su toque de variabilidad, no lo sabemos sino hasta que lo estudiamos; es así que, de acuerdo al tipo de cancha, las velocidades son diferentes, así como también, los tiempos de trabajo y pausa, las distancias recorridas, los desplazamientos de acuerdo al porcentaje de utilización, la altura de bote de la pelota y por consiguiente el golpeo de la misma.

Según nos hemos podido dar cuenta en los estudios realizados a los diferentes partidos $y$ en los diferentes tipos de cancha, hemos observado con detenimiento cada uno de los desplazamientos de los jugadores y jugadoras, y hemos determinado bajo tablas de análisis que: los tipos de desplazamientos más utilizados son los "desplazamientos de frente". Si bien hemos podido encontrar en la bibliografía algunas referencias en cuanto a los tipos de desplazamientos más realizados en el tenis, encontramos que según, Marks, Kovacs, los desplazamientos más realizados en el tenis son los desplazamientos “laterales”, lo mismo propone, Vila G, Carlos. Esto, no sucede así en nuestra investigación.

Con lo anterior, desde nuestra tesis, hemos sacado la conclusión también, bajo la observación minuciosa de cada desplazamiento; que la mayoría de desplazamientos que se hacen en un partido no son de forma lateral, pues los estudios que hemos hecho, demuestran lo contrario; quizás porque estos estudios de los autores anteriormente registrados, tienen algunos años de haberse realizado o la pauta para designar un tipo de desplazamiento es diferente. En esta tesis, la configuración que utilizamos para designar al desplazamiento "de frente", es la de ir hacia el frente, sin importa la dirección ni la ubicación en la cancha; el jugador se mueve hacia adelante como la mecánica de la carrera o del caminar. 
Si bien podríamos pensar que el jugador se traslada de forma lateral por el hecho de estar situado detrás de la línea de fondo de la cancha y que allí es donde se realizan la mayor parte de desplazamientos y golpeos; según nuestros estudios, no es así, porque el deportista o la deportista cuando necesita trasladarse a cierta distancia (ej. $2 \mathrm{~m}, 4 \mathrm{~m} \circ 6 \mathrm{~m}$ ) lo va a hacer de forma frontal. Esto puede deberse, sin embargo, a la mecánica de la competencia en el tenis, pues la pelota viaja a gran velocidad y en diferentes direcciones, por tal motivo, si el deportista se traslada de frente, lo hace a mayor velocidad y llega a pelotas muy lejanas de su cuerpo que probablemente si se desplaza de lado no podrá llegar en el momento exacto.

En segundo lugar, tenemos los desplazamientos laterales de recuperación ( $L r)$; estos desplazamientos son utilizados siempre que se termina de golpear, para luego retomar la posición de donde había partido u otra de acuerdo a como crea que va a venir la pelota del otro lado (anticipación). En las observaciones que se hicieron a todos los partidos de tenis, estos desplazamientos se realizaban con un pie al lado de otro o pasando un pie por delante del otro; cada jugador o jugadora utilizaba estas dos formas de desplazamiento lateral para recuperar la posición donde estaban.

Por último, encontramos que los desplazamientos hacia arriba, donde se pueden observar "servicios o Smash", el porcentaje de utilización de este tipo de desplazamiento es importante, siempre con un valor de $16 \%$ o 18\% del total de todos los tipos de golpes, eso quiere decir, que sobrepasa en número los desplazamientos hacia atrás para golpear o para recuperar y, casi que iguala en porcentaje a los tipos de desplazamientos de lado para golpear. Los Smash en cambio, se ha observado que su participación en el juego es casi nula, encontrando solo uno o dos golpes de este tipo en un set. Aunque no medimos cada trayectoria de cada servicio, sí lo tenemos como referencia en cuanto a tipo de desplazamiento pasa saber su proporción de utilización y tenerlo en cuenta para pensar cuanto deberíamos dedicarle a este tipo de golpe. 


\subsection{Distancias recorridas en la competición.}

Las distancias recorridas en el tenis las podemos clasificar según su longitud y según su frecuencia de utilización en un partido de tenis. Estas distancias nos van a dar un conocimiento valedero para tener en cuenta el estudio de las mismas y para cualquier propuesta que se haga a partir de allí. Para eso vamos a tener en cuenta en los siguientes párrafos, cuáles son las distancias más recorridas, cuáles son las más largas y cuáles son las más cortas de acuerdo con cada tipo de desplazamiento.

\subsubsection{Distancias cortas más recorridas.}

Las distancias cortas más recorridas en el tenis, según nuestra investigación, nos arroja el resultado que son las de 1, 2 y 3 metros. Estas distancias recorridas por los jugadores y jugadoras, están sometidas a muchos factores, como dimensiones de la cancha, velocidad de la pelota, anticipación, desplazamientos rápidos y ajustados. Pero si bien es así que un desplazamiento de lado para hacer dos metros es rápido, no representa mayor ajuste para el deportista, por ende, se ha observado que prefieren hacerlo de frente para llegar con buena velocidad y apoyarse, golpear y recuperar la posición si es necesario, pero de forma lateral, que es más lento, pero da apoyo para un repentino cambio de dirección.

Las distancias de $1 \mathrm{~m}$, en muchas ocasiones, se realizan de forma lateral, proporcionando al jugador un paso menos para recuperar la posición. Las distancias de $2 \mathrm{~m}$ son más rápidas hacerlas de frente, y aportan más estabilidad al deportista para llegar, plantarse y desde allí golpear y recuperar la posición. Por ultimo tenemos las distancias de $3 \mathrm{~m}$, estas distancias, mayormente se hacen de forma frontal cuando se va a impactar la pelota por la velocidad en que viene y por la velocidad en que el deportista se puede desplazar hacia adelante. Los desplazamientos laterales de recuperación en estas distancias, si bien es la misma distancia, ayudan a recuperar la posición, da mayor campo visual, mayor facilidad de cambiar de dirección y siempre queda en frente del rival. 


\subsubsection{Distancias largas más recorridas.}

Las distancias largas son propias de los desplazamientos frontales o hacia delante en cualquier dirección y nunca se hacen de forma lateral, pues desplazarse de forma lateral es demasiado lento y en el tenis de hoy la velocidad es un gran aliado. Estas distancias, en su gran mayoría, aunque son de $4 \mathrm{~m}$, pero podemos encontrar que también hay un pequeño número de distancias recorridas entre $5 \mathrm{~m}$ y $8 \mathrm{~m}$, comparada con la escasa y casi nula distancia de $12 \mathrm{~m}$ o $24,81 \mathrm{~m}$ que es lo máximo que un tenista podría recorrer en una cancha de tenis en línea recta. Estas distancias de $4 \mathrm{~m}$ a $8 \mathrm{~m}$, se realizan siempre hacia los costados de la cancha o hacia la red en una dejada.

En número de veces, encontramos que la distancia larga más recorrida es la de $4 \mathrm{~m}$, seguido por la de $5 \mathrm{~m}$, después la de $6 \mathrm{~m}$ y así sucesivamente. Encontramos también que en los partidos pueden recorrer pocas veces los $8 \mathrm{~m}$ o 10m, 1, 2 o 3 veces en un set; esto sucede cuando el jugador tiene que ir a responder a un lado extremo de la cancha a $1 \mathrm{~m}$ o $2 \mathrm{~m}$ detrás de la línea de fondo, y el contrincante hace un Drop cerca a la red; o, cuando un jugador es obligado a ir a un lado extremo de la cancha paralelo a la línea de fondo, e inmediatamente tiene que ir al lado opuesto a responder otra pelota. En estos momentos los desplazamientos son como lo decía anteriormente "de frente" y a máxima velocidad.

\subsection{Tiempos verdaderos de trabajo-pausa en la competición.}

Como nos hemos podido dar cuenta en los cuadros de estudio que hicimos a los partidos de tenis, encontramos que, dependiendo el tipo de superficie de la cancha, el punto se hace más largo o más corto. Si bien, existe la capacidad de los jugadores de primer nivel, mantener la pelota en juego por largo rato y a una alta intensidad, las características de la cancha hacen que no se pueda mantener un largo peloteo; bien porque la pelota salta mucho, porque la pelota bota bajo o simplemente porque la pelota después del pique viaja muy rápido. En los siguientes párrafos, vamos a hablar de los tiempos globales, específicos y por punto, en cada uno de los tipos de superficies. 
Los peloteos más largos en tiempo, están registrados en canchas de superficie lenta (polvo de ladrillo). Estas superficies hacen que la pelota al momento de botar, reduzca su velocidad y de más tiempo de acomodación al deportista o lo exija más para que tenga que buscar lugares más alejados de la cancha para poder impactar. Los tiempos manejados en estos tipos de pistas de acuerdo a la relación trabajo-pausa, están en promedio de 8" a 9" de trabajo y de 24 " a 25 " de descanso, eso quiere decir que en promedio se trabaja 1" por 2,5" de pausa. Esto lo estamos hablando desde los tiempos más específicos sin tener en cuenta los grandes momentos de descanso que se tiene al cambiar de lado; pero si tomáramos un tiempo global, la relación de trabajo-pausa sería de 1" de trabajo por 3,5" de pausa; que en realidad varía un segundo, pero es significativo para tenerlo en cuenta a la hora de planificar un entrenamiento.

Por otro lado, la mayoría de los torneos alrededor del mundo se juegan en cancha duras o "de cemento", sea Indoor u Outdoor. Las características de estos tipos de superficies para el juego son de velocidad media, eso quiere decir que está entre la velocidad lenta del polvo de ladrillo y la velocidad rápida del césped. Ahora, los tiempos de trabajo-pausa manejados en estas cachas son diferentes a los de las demás, encontrándonos que en comparación con la de polvo de ladrillo el tiempo de trabajo se reduce, pero el tiempo de descanso se mantiene y se tiende a elevar; esto conlleva a que trabajen menos y descansen más en comparación con la de polvo de ladrillo, podría ser a lo sumo por el trabajo mecánico de tracción en los cambios de dirección generando más impacto las canchas duras, que en las de polvo de ladrillo. Encontramos entonces, que los tiempos globales en cancha dura para hombres y mujeres son de 1" de trabajo por 4,65" de descanso, los tiempos específicos 1 " de trabajo por 3,50" de descanso, y el promedio entre punto y punto es de 7" de trabajo por 25 " de pausa.

Por último, tenemos el tipo de pista más rápida, cancha de hierba o césped. Como bien lo dice la ITF y el estudio realizado en el capítulo IV, las características de la cancha hacen que sea la más rápida de todas. Aunque hay pocos torneos que tengan estos tipos de superficies, no podemos obviar y 
dejar de lado lo que ocurre allí. Ahora bien, en cuanto a los tiempos de trabajopausa que se lograron estudiar en esta investigación, tienen una característica muy importante: es la superficie donde menos se trabaja y más se descansa en comparación con los otros dos tipos de canchas (polvo de ladrillo y cemento). Observamos que el tiempo promedio global es de 1" de trabajo por 5,5" de descanso, el promedio específico es de 1" de trabajo y 4" de descanso y el promedio punto por punto es de 5" por 19,5" de descanso; esto para hombre y mujeres, que coincidencialmente se presenta así en cada uno de los tipos de superficie con alguna diferencia en promedio de 0,5 " a 1 ".

\subsection{Promedios y relación de utilización de los tipos de golpes en el "peloteo previo".}

Como pudimos observar en el capítulo pasado, los jugadores y jugadoras entraban a la cancha y después de los actos protocolarios, realizaban un "peloteo previo" al partido, esto, para alistar todos sus golpes y entrar en ritmo de juego para que a la hora de empezar el juego estén en óptimas condiciones. Ahora bien, este momento nos da un click para pensar por qué lo hacen y porque manejan sistemáticamente los mismos golpes en cada fase y con el mismo tiempo promedio para cada golpe. Hemos encontrado entonces una relación entre los porcentajes de utilización de los tipos de desplazamientos y éste momento que utilizan las jugadoras y jugadores antes de empezar el partido.

Es así que destinan la mayor cantidad de tiempo y de número de ejecuciones para los golpes de la "derecha y el revés" en el fondo de la cancha, con un promedio de 145 " que sería un $43 \%$ del total de los tipos de golpe, y solo un poco más el "servicio", con un 146,2 " que es un $43,55 \%$. Por otro lado, tenemos los otros dos tipos de golpes, la "volea" con un promedio de 34,5 " que es un $9,59 \%$ y el Smash con un promedio de 13 " que es un $3,77 \%$. Si eso lo comparamos con los porcentajes de utilización de los tipos de desplazamientos, encontramos una gran similitud, pues en los desplazamientos frontales y laterales se realizan "el revés y la derecha" y estos son los de mayor utilización en un partido, al igual que el servicio, que es un desplazamiento 
hacia arriba que siempre está presente una o dos veces al comenzar un punto; en cambio la volea y el smash, son movimientos que muy pocas veces se ejecutan en un partido. 


\section{ACONDICIONAMIENTO FÍSICO PRECOMPETITIVO PARA EL TENIS.}

\subsection{Acondicionamiento Físico Precompetitivo par el Tenis.}

El "Acondicionamiento Físico Precompetitivo para el Tenis", nace de la necesidad de encontrar una propuesta que ayude a que los deportistas se preparen de la mejor manera para la competencia (partido de tenis). Esto, basado en el estudio específico de los momentos de trabajo-pausa, de los tipos de desplazamientos y las distancias recorridas en cada punto, que, en definitiva, atienden a la especificidad del deporte para hacer una propuesta acorde a estas necesidades propias de la competición. Para ello, propondremos desde un enfoque metodológico, teniendo en cuenta todos los promedios de los estudios anteriormente dichos, en cada tipo de superficie, para hombres y para mujeres en competiciones de alto nivel.

Después de haber recolectado bastante información sobre el desarrollo de las competiciones entre hombres y entre mujeres, esos datos lo vamos a tener en cuenta para desarrollar la propuesta que desde aquí en adelante iremos a desarrollar. Este no será, por ningún motivo, una propuesta inamovible, será pues una propuesta inclusiva y de posible adaptación a cualquier tipo de jugador y a cualquier tipo de torneo donde haya un tipo de superficie en la cual investigamos y tenemos conocimientos; sin embargo, será también un punto de partida para repensar inclusive, el entrenamiento de los tenistas, como también su preparación para el acto competitivo.

En las siguientes páginas vamos a leer algunos argumentos sustraídos de la investigación, que se deben tener en cuenta para las propuestas que se hacen a partir de: la especificidad en los desplazamientos y sus distancias, especificidad en tiempos de trabajo pausa y, por último, una metodología para desarrollar estas especificidades en el "Acondicionamiento Físico Precompetitivo para el tenis". 


\subsubsection{Especificidad en tipos de desplazamientos y sus distancias para el Acondicionamiento Físico Precompetitivo}

Como ya hemos dicho, y basado en las investigaciones que se le hicieron a cada partido entre hombres y mujeres en torneos de alto nivel, y en diferentes tipos de superficie (polvo de ladrillo, césped y cemento), encontramos que hay una relación porcentual en el número de utilización de los tipos de desplazamientos. Es así que los desplazamientos hacia adelante son los que más se realizan, le siguen los desplazamientos laterales de recuperación y después los desplazamientos hacia arriba o servicios. En este orden de jerarquía, se propone que se dé importancia para preparar al deportista antes de la competencia, es decir, cómo se debe acondicionar físicamente al deportista en: repeticiones de desplazamientos, distancias de estos desplazamientos junto con los tiempos de trabajo-pausa.

Para poder observar según el orden de importancia en cada tipo de desplazamiento, en la tabla 109, vamos a poder apreciar los promedios y porcentajes, según se ha realizado el estudio del capítulo IV para cada tipo de desplazamiento en la presente tesis. Estos resultados se deben tener en cuenta para tomar como base razonable en una hipotética planificación de un "acondicionamiento físico pre competitivo" a cualquier deportista para cualquier tipo de superficie en la que quiera jugar.

\begin{tabular}{|c|c|c|c|c|c|}
\hline \multicolumn{3}{|c|}{$\begin{array}{c}\text { PROMEDIO PONDERADO } \\
\text { NÚMERO DE VECES }\end{array}$} & \multicolumn{3}{c|}{$\begin{array}{c}\text { PROMEDIO PONDERADO DE } \\
\text { DISTANCIAS }\end{array}$} \\
\hline & Hombres & Mujeres & & Hombres & Mujeres \\
\hline F & $39,69 \%$ & $38,76 \%$ & $\mathrm{~F}$ & $58,48 \%$ & $59,62 \%$ \\
\hline A & $6,57 \%$ & $6,24 \%$ & A & $6,35 \%$ & $5,26 \%$ \\
\hline AA & $6,46 \%$ & $11,57 \%$ & AA & $3,62 \%$ & $7,91 \%$ \\
\hline L & $10,48 \%$ & $7,94 \%$ & L & $8,36 \%$ & $5,98 \%$ \\
\hline Lr & $23,07 \%$ & $20,73 \%$ & Lr & $22,81 \%$ & $21,18 \%$ \\
\hline S & $14,67 \%$ & $14,75 \%$ & & & \\
\hline
\end{tabular}

Tabla 109.Promedio ponderado de desplazamientos En los tres Tipos de superficie.

Agregando a lo anterior, en el capítulo IV, observamos laS distancias que más se realizan haciendo desplazamientos hacia adelante para impactar la pelota, y encontramos que para las pistas de césped son de $2 \mathrm{~m}$ en hombres y mujeres; 
para las de polvo de ladrillo son de $2 \mathrm{~m}$ para hombres y de $1 \mathrm{~m}$ para mujeres, $\mathrm{y}$, en pista dura, son de $1 \mathrm{~m}$ para hombres y mujeres respectivamente.

Para los desplazamientos hacia "atrás de ejecución", tenemos que, para las pistas de césped, la mayoría de distancia recorrida es de $1 \mathrm{~m}$ para las mujeres y $2 \mathrm{~m}$ para hombres, en pistas de polvo de ladrillo son de $2 \mathrm{~m}$ para hombres y $1 \mathrm{~m}$ para mujeres, para pistas duras son de $1 \mathrm{~m}$ para hombres y mujeres respectivamente.

En cuanto a los desplazamientos "hacia atrás de recuperación", en pistas de césped son de $1 \mathrm{~m}$ para hombres y mujeres respectivamente, en pistas de polvo de ladrillo son de $1 \mathrm{~m}$ también para hombres y mujeres, así como en las pistas duras de $1 \mathrm{~m}$ para hombres y mujeres respectivamente, encontrándose más coincidencia en este tipo de desplazamientos para los tres tipos de superficies al igual que vamos a encontrar en el siguiente párrafo para los otros tipos de desplazamientos.

Los tipos de desplazamientos laterales como ya habíamos dicho antes, tiene sus dos variantes, por tal motivo, en cuanto a los "laterales de ejecución", encontramos que recorren la misma distancia de $1 \mathrm{~m}$ el mayor número de veces $y$, para los desplazamientos "laterales de recuperación" tenemos que las distancia más recorridas es de $2 \mathrm{~m}$ respectivamente para hombres y mujeres en los tres tipos de pista. En cuanto a los desplazamientos hacia arriba, en el cual el $99 \%$ son servicios, ya tenemos los porcentajes en la tabla 109 y que su rango de prioridad de utilización es el tercero.

Para terminar, con toda esta información tenemos un referente para preparar los/as tenistas en cuanto a los tipos de desplazamientos y las distancias que más se necesitan preparar para la competencia. No queremos decir con esto que la preparación del jugador/a se base solamente en estas distancias anteriormente dichas, sino que son las más utilizadas y que es un punto de partida para tener en cuenta. Podemos encontrar el promedio más completo de los distintos tipos de desplazamientos en cada superficie en el capítulo IV en los cuadros de estudio y resultados de los partidos. Entonces, el objetivo de 
esta propuesta, apuntaría a trata de hacer más específica la preparación del deportista para cada tipo de superficie.

\subsubsection{Especificidad en tiempos de trabajo-pausa para el Acondicionamiento Físico Precompetitivo.}

En cuanto a los tiempos de trabajo y pausa, también se hacen muy importantes tenerlos en cuenta para la preparación de los deportistas, pues desde allí estamos atendiendo tanto a los tiempos específicos de una competencia, como también a los sistemas energéticos, es decir, al perfil fisiológico del/a tenista en este deporte. $\mathrm{Y}$ es que, si una preparación no se hace con los tiempos específicos que se manejan en el tenis, estaríamos preparando al deportista para otra actividad y no para la competencia en las canchas de tenis.

La preparación de un/a tenista debe hacerse de acuerdo a cada tipo de competición, pues dependiendo el tipo de pista a la cual se vaya a competir, los tiempos de trabajo y pausa varían. Es así que para una competición en pista de polvo de ladrillo los tiempos de trabajo son superiores a los de las otras pistas, teniendo en promedio 8" a 9" tanto para hombres como para mujeres en puntos jugados; no así en la pista de césped que es totalmente contrario, siendo la pista en la que el promedio de trabajo es el más bajo de todas las pistas, encontrando que los tiempos están entre 4,17" para mujeres y 5,77" para hombres, donde solo los separa un segundo de diferencia en promedio de trabajo; y por último, tenemos que en las canchas duras los tiempos están en la mitad de las dos canchas anteriores en cuanto a tiempo de "trabajo" se refiere, tenemos que está entre 6" y 7,50" para hombres y mujeres.

Ahora, si se está preparando a un deportista para una competencia, también se debe tener en cuenta los tiempos de descanso para adaptar el organismo a dichos momentos de recomposición de energía potencial. Y si vemos, aunque los tiempos por reglamento (Reglas ITF 2014. 19) son de 20" de descanso entre punto, 90" en cambio de lado y de 120" para el cambio de set; vemos que no se respetan estos tiempos, se toman mucho más tiempo, algo que tenemos que tener en cuenta para la preparación de los deportistas, pues, hemos 
encontrado que los tiempos de descanso en promedio varía entre los 19" y 26" aproximadamente. Otra relación para tener en cuenta es que en las canchas de polvo de ladrillo y de cemento, están entre 24" y26", para mujeres y hombres respectivamente, no así en las de césped que el promedio está entre 19" y 20" para ambos géneros.

En relación con lo anterior, tenemos que tener en cuenta también que la cancha en que más se trabaja y menos se descansa es la cancha de polvo de ladrillo, luego sigue la de cemento y por último tenemos que en la de césped se trabaja menos y se descansa más. Este mayor o menor descanso está relacionado con el tiempo de trabajo de ese tipo de pista. Ahora bien, ya teniendo toda esta información valiosa de lo que pasa y lo que debemos tener en cuenta para preparar los deportistas; las sesiones de "Acondicionamiento Físico Precompetitivo para el Tenis" deben respetar estos tiempos de trabajo y pausa para cada tipo de superficie, y así responder a las necesidades propias y específicas que se van a presentar en una competencia.

\subsubsection{Aspectos metodológicos a tener en cuenta para una realización de Acondicionamiento Físico Precompetitivo.}

Antes de una competición, los deportistas deben prepararse específicamente para lo que viene. Es así, que los entrenadores o preparadores físicos deben tener en cuenta a qué tipo de superficie se enfrentan y cuáles son los tiempos de trabajo y pausa que promediamente se presentan allí, como también los promedios de distancias de todos los tipos de desplazamientos y por último, incluir la característica propia del jugador, pues cada deportista tiene, por ejemplo, un tipo de desplazamiento diferente para impactar una pelota que viene del otro lado a una velocidad muy baja y alta; otro ejemplo es reducir un tipo de desplazamiento o sustituirlo por otro para realizar un contragolpe.

Los tiempos de preparación de un deportista en el "Acondicionamiento Físico Precompetitivo para el Tenis", pueden tardar entre 45' y una hora para tener el tiempo necesario de preparación, de forma progresiva y respetando los tiempos específicos de trabajo-pausa para que no se caiga en el agotamiento. Se 
empezaría entonces, con los estiramientos activos a intensidad media "haciendo los gestos de desplazamientos y golpeos" preparando el sistema muscular, y óseo para la acción (10 a 15 minutos). Después, hacer ejercicios de pasadas con todos los tipos de desplazamientos, haciendo hincapié en lo que más se van a utilizar en la competencia y según la característica del jugador, teniendo en cuenta también las distancias y a intensidad parecida al juego" junto con todos los tipos de golpes (40 a 50 minutos).

No se hará ningún tipo de estiramiento al finalizar el "Acondicionamiento Físico Precompetitivo para el Tenis" y se ingresará así tan pronto sea llamado a la cancha. Todo esto como lo decía en párrafos anteriores, respetando los tiempos de trabajo y pausa ya descritos para cada tipo de superficie y, teniendo en cuenta también si es para hombres o para mujeres, pues se logra apreciar algunas diferencias.

El porcentaje para la preparación de cada tipo de desplazamiento y cada tipo de golpe; debe guardar relación con lo que en promedio se va a utilizar en cada tipo de superficie, guardando relación siempre que sea posible con las propuestas anteriores en relación a promedios y porcentajes de trabajo pausa.

Con todo lo anterior y, para terminar; si se logra cumplir o realizar a cabalidad con las propuestas anteriormente dichas, el jugador/a entrará a la cancha, habiendo hecho el "Acondicionamiento Físico Precompetitivo para el Tenis", alistando todas sus capacidades específicas deportivas en un rendimiento superlativo y listo para jugar al tenis. 


\section{BIBLIOGRAFÍA:}

Bompa, T. (2004). PERIODIZACIÓN DEL ENTRENAMIENTO DEPORTIVO (PROGRAMA PARA OBTENER EL MÁXIMO RENDIMIENTO EN 35 DEPORTES). Editorial Paidotrivo. Barcelona España.

Blázquez, D. (2004). EL CALENTAMENTO, UNA VÍA PARA LA AUTOGESTIÓN DE LA ACTIVIDAD FÍSICA. Editorial Inde. Barcelona España.

Fernández, J. (2009). VALORACIÓN DE LA CONDICIÓN FÍSICA DEL ALUMNADO DEL CEIP O GRUPO DE RIBEIRA MEDIANTE LA BATERÍA EUROFIT. Editorial Lulu Enterprice. United Kindom.

Foran, B. (2007). ACONDICIONAMIENTO FÍSICO PARA DEPORTES DE ALTO RENDIMIENTO. Editorial Hispano Europea SA. Barcelona España.

ITF RÉGLES DU TENNIS. (2014). INTERNATIONAL TENNIS FEDERATION.

Alvares, J. (2011). CALENTAMIENTO Y VUELTA A LA CALMA EN EL TENIS. Tomado de: http://www.itftennis.com/media/113772/113772.pdf.

Granell, J. Cervera, V. (2003). TEORÍA Y PLANIFICACIÓN DEL ENTRENAMIENTO DEPORTIVO. Editorial Paidotrivo. Barcelona España.

Jiménez, F. (2008). EL ACONDICIONAMIENTO FÍSICO ORIENTADO A LA SALUD EN LA ESO. Editorial Asociación Procompal. Barcelona España.

Kovacs s, Marcs. (2014). MOVIMIENTOS DEL TENIS: LA IMPORTANCIA DE ENTRENAMIENTO LATERAL. Revista de entrenamiento deportivo. http://gse.com/es/journals/revistaentrenamientodeportivo/articulos/movimientos-deltenis-la-importancia-del-entrenamiento-lateral-1172.

Le Deuff, H. (2005). EL ENTRENAMIENTO FÍSICO DEL JUGADOR DE TENIS. Editorial Paidotrivo. Badalona España. 
Naclerio, F. (2011). ENTRENAMIENTO DEPORTIVO, FUNDAMENTOS Y APLICACIONES EN DIFERENTES DEPORTES. Editorial Médica Panamericana. Madrid España.

Ortiz, R. (2004). TENIS, POTENCIA, VELOCIDAD Y MOVILIDAD. Editorial Inde. Barcelona España.

Platonov, V. (2001). TEORÍA GENERAL DEL ENTRENAMIENTO DEPORTIVO OLÍMPICO. Editorial Paidotrivo, Barcelona España.

Reid, M, Quinn A y Crespo M. (2003). ITF FUERZA Y CONDICIÓN FÍSICA PARA EL TENIS. Editado por Machar Reid, AnneQuinn y MiguelCrespo. España.

Roetert, P. Ellenbecker, T. (2000). PREPARACIÓN FÍSICA COMPLETA PARA EL TENIS -UNITED STATES TENNIS ASSOCIATION-. Editorial ediciones Tutor S.A. Madrid España.

Siff, M. Verkhoshansky, Y. (2000). SUPERENTRENAMIENTO. Editorial Paidotrivo. Barcelona España.

Vídeo. FINAL DEL AUSTRALIAN OPEN 2014. ENTRE DOMINICA CIBULKOVA VS LI NA. https://www.youtube.com/watch?v=a1M2qr1Ipg8.

Vídeo. FINAL MONTECARLO ROLEX 2014. ENTRE RAFAEL NADAL VS NOVAK DJOKOVIC. https://www.youtube.com/watch?v=W1fIApf4JMQ.

Vídeo. FINAL WIMBLEDON 2014. ENTRE EUGÉNIE BOUCHARD VS PETRA KVITOVA. https://www.youtube.com/watch?v=PMXcrBIL5x0.

Vídeo. SEMIFINAL COPA CLARO 2013. ENTRE PAULA ORMAECHEA VS TELIANA PEREIRA. https://www.youtube.com/watch?v=1Ein3ezYFcU.

Vídeo. FINAL WIMBLEDON 2014. ENTRE NOVAK DJOKOVIC VS ROGER FEDERER. https://www.youtube.com/watch?v=m-h0Qc935G8

Weineck, J. (2005). ENTRENAMIENTO TOTAL. Editorial Paidotrivo. Barcelona España. 